\title{
HDV/ $/$ SURVELLANGE
}

Volume 28

\section{Diagnoses of HIV Infection in the United States and Dependent Areas, 2016}


The HIV Surveillance Report is published annually by the Division of HIV/AIDS Prevention, National Center for HIV/AIDS, Viral Hepatitis, STD, and TB Prevention, Centers for Disease Control and Prevention (CDC), U.S. Department of Health and Human Services, Atlanta, Georgia.

Data are presented for diagnoses of HIV infection reported to CDC through June 2017.

The HIV Surveillance Report is not copyrighted and may be used and reproduced without permission. Citation of the source is, however, appreciated.

\section{Suggested citation}

Centers for Disease Control and Prevention. HIV Surveillance Report, 2016; vol. 28.

http://www.cdc.gov/hiv/library/reports/hiv-surveillance.html. Published November 2017. Accessed [date].

On the Web: http://www.cdc.gov/hiv/library/reports/hiv-surveillance.html

\section{Confidential information, referrals, and educational material on HIV infection}

CDC-INFO

1-800-232-4636 (in English, en Español)

$1-888-232-6348$ (TTY)

http://wwwn.cdc.gov/des/ContactUs/Form

\section{Acknowledgments}

Publication of this report would not have been possible without the contributions of the state and territorial health departments and the HIV surveillance programs that provided surveillance data to CDC.

This report was prepared by the following CDC staff and contractors: Kristen Hess, Anna Satcher Johnson, Xiaohong $\mathrm{Hu}$, Jianmin Li, Baohua Wu, Chenchen Yu, Hong Zhu, Chan Jin, Mi Chen, John Gerstle, Marie Morgan, Michael Friend, Azfar Siddiqi, Angela Hernandez, the HIV Incidence and Case Surveillance Branch, and the Data Management Team of the Quantitative Sciences and Data Management Branch. 


\section{Commentary}

Tables

\section{Section 1 Diagnoses of HIV Infection and Diagnoses of Infection Classified as Stage 3 (AIDS)}

1a Diagnoses of HIV infection, by year of diagnosis and selected characteristics, 2011-2016-United States

$1 b$ Diagnoses of HIV infection, by year of diagnosis and selected characteristics, 2011-2016 - United States and 6 dependent areas

2a Stage 3 (AIDS), by year of diagnosis and selected characteristics, 2011-2016 and cumulative-United States

2b Stage 3 (AIDS), by year of diagnosis and selected characteristics, 2011-2016 and cumulative-United States and 6 dependent areas

3a Diagnoses of HIV infection, by race/ethnicity and selected characteristics, 2016-United States

3b Diagnoses of HIV infection, by race/ethnicity and selected characteristics, 2016-United States and 6 dependent areas

4a Stage 3 (AIDS), by race/ethnicity and selected characteristics, 2016-United States

$4 \mathrm{~b}$ Stage 3 (AIDS), by race/ethnicity and selected characteristics, 2016-United States and 6 dependent areas

5a Diagnoses of HIV infection attributed to male-to-male sexual contact and male-to-male sexual contact and injection drug use, by selected characteristics, 2011-2016 - United States

$5 \mathrm{~b}$ Diagnoses of HIV infection attributed to male-to-male sexual contact and male-to-male sexual contact and injection drug use, by selected characteristics, 2011-2016-United States and 6 dependent areas

6a Diagnoses of HIV infection attributed to injection drug use, by selected characteristics, 2011-2016United States

6b Diagnoses of HIV infection attributed to injection drug use, by selected characteristics, 2011-2016-United States and 6 dependent areas

7a Diagnoses of HIV infection attributed to heterosexual contact, by selected characteristics, 2011-2016United States

7b Diagnoses of HIV infection attributed to heterosexual contact, by selected characteristics, 2011-2016-United States and 6 dependent areas

8a Stage 3 (AIDS) attributed to male-to-male sexual contact and male-to-male sexual contact and injection drug use, by selected characteristics, 2011-2016-United States

$8 \mathrm{~b}$ Stage 3 (AIDS) attributed to male-to-male sexual contact and male-to-male sexual contact and injection drug use, by selected characteristics, 2011-2016-United States and 6 dependent areas

9a Stage 3 (AIDS) attributed to injection drug use, by selected characteristics, 2011-2016-United States

9b Stage 3 (AIDS) attributed to injection drug use, by selected characteristics, 2011-2016-United States and 6 dependent areas

10a Stage 3 (AIDS) attributed to heterosexual contact, by selected characteristics, 2011-2016 - United States

$10 \mathrm{~b}$ Stage 3 (AIDS) attributed to heterosexual contact, by selected characteristics, 2011-2016-United States and 6 dependent areas

11a Diagnoses of HIV infection among children aged $<13$ years, by race/ethnicity, 2011-2016 - United States

11b Diagnoses of HIV infection attributed to heterosexual contact, by selected characteristics, 2011-2016-United States and 6 dependent areas

12a Stage 3 (AIDS) among children aged $<13$ years, by race/ethnicity, 2011-2016 and cumulative - United States

12 b Stage 3 (AIDS) among children aged $<13$ years, by race/ethnicity, 2011-2016 and cumulative — United States and 6 dependent areas

13 Stage 3 (AIDS) among children aged $<13$ years, by year of diagnosis, 1992-2016 - United States and 6 dependent areas

14 Diagnoses of HIV infection among adult and adolescent Hispanics/Latinos, by transmission category and place of birth, 2016-United States and 6 dependent areas

15 Stage 3 (AIDS) among adult and adolescent Hispanics/Latinos, by transmission category and place of birth, 2016-United States and 6 dependent areas 
Section 2 Deaths and Survival after a Diagnosis of HIV Infection or Stage 3 (AIDS) Classification

16a Deaths of persons with diagnosed HIV infection, by year of death and selected characteristics, 2011-2015United States

16b Deaths of persons with diagnosed HIV infection, by year of death and selected characteristics, 2011-2015United States and 6 dependent areas

17a Deaths of persons with diagnosed HIV infection ever classified as stage 3 (AIDS), by year of death and selected characteristics, 2011-2015 and cumulative-United States

17b Deaths of persons with diagnosed HIV infection ever classified as stage 3 (AIDS), by year of death and selected characteristics, 2011-2015 and cumulative-United States and 6 dependent areas

18a Deaths of persons with diagnosed HIV infection, by race/ethnicity and selected characteristics, 2015United States

$18 \mathrm{~b}$ Deaths of persons with diagnosed HIV infection, by race/ethnicity and selected characteristics, 2015United States and 6 dependent areas

19a Deaths of persons with diagnosed HIV infection ever classified as stage 3 (AIDS), by race/ethnicity and selected characteristics, 2015-United States

$19 \mathrm{~b}$ Deaths of persons with diagnosed HIV infection ever classified as stage 3 (AIDS), by race/ethnicity and selected characteristics, 2015-United States and 6 dependent areas

\section{Section 3 Persons Living with Diagnosed HIV Infection or with Infection Ever Classified as Stage 3 (AIDS) (Prevalence)}

20a Persons living with diagnosed HIV infection, by year and selected characteristics, 2011-2015-United States

20b Persons living with diagnosed HIV infection, by year and selected characteristics, 2011-2015-United States and 6 dependent areas

21a Persons living with diagnosed HIV infection ever classified as stage 3 (AIDS), by year and selected characteristics, 2011-2015-United States

21b Persons living with diagnosed HIV infection ever classified as stage 3 (AIDS), by year and selected characteristics, 2011-2015-United States and 6 dependent areas

22a Persons living with diagnosed HIV infection, by race/ethnicity and selected characteristics, year-end 2015United States

22b Persons living with diagnosed HIV infection, by race/ethnicity and selected characteristics, year-end 2015United States and 6 dependent areas

23a Persons living with diagnosed HIV infection ever classified as stage 3 (AIDS), by race/ethnicity and selected 106 characteristics, year-end 2015-United States

23b Persons living with diagnosed HIV infection ever classified as stage 3 (AIDS), by race/ethnicity and selected 108 characteristics, year-end 2015-United States and 6 dependent areas

\section{Section 4 Data by Area of Residence}

24 Diagnoses of HIV infection, by area of residence, 2015 and 2016-United States and 6 dependent areas

25 Stage 3 (AIDS), by area of residence, 2015, 2016, and cumulative - United States and 6 dependent areas

26 Adults and adolescents living with diagnosed HIV infection, by race/ethnicity and area of residence, year-end 113 2015-United States and 6 dependent areas

27 Adults and adolescents living with diagnosed HIV infection ever classified as stage 3 (AIDS), by race/ethnicity 115 and area of residence, year-end 2015-United States and 6 dependent areas

28 Diagnoses of HIV infection, 2016, and persons living with diagnosed HIV infection (prevalence), year-end 2015, by metropolitan statistical area of residence-United States and Puerto Rico

29 Stage 3 (AIDS), 2016 and cumulative, and persons living with diagnosed HIV infection ever classified as stage 3 (AIDS) (prevalence), year-end 2015, by metropolitan statistical area of residence-United States and Puerto Rico

Web Addresses for Reports of State and Local HIV Surveillance 


\section{Commentary}

The Centers for Disease Control and Prevention (CDC) collects, analyzes, and disseminates surveillance data on HIV infection; these data are one of the nation's primary sources of information on HIV in the United States. The annual surveillance report, published by the Division of HIV/AIDS Prevention, summarizes information about diagnosed HIV infection in the United States and dependent areas. HIV surveillance data are used by CDC's public health partners in other federal agencies, health departments, nonprofit organizations, academic institutions, and the general public to help focus prevention efforts; plan services; allocate resources; develop policy; detect, monitor, and intervene in HIV clusters; and monitor trends in HIV infection.

Numbers and rates of diagnoses of HIV infection during 2011-2015 and preliminary numbers for 2016 are based on data from all 50 states, the District of Columbia, and 6 U.S. dependent areas (American Samoa, Guam, the Northern Mariana Islands, Puerto Rico, the Republic of Palau, and the U.S. Virgin Islands).

During 2011-2015, the annual number and rate of diagnoses of HIV infection in the United States decreased. Numbers and rates of diagnoses of HIV infection increased in some subgroups and decreased in others. Variations in trends among groups are expected and may be due to differences in testing behaviors, targeted HIV testing initiatives, or changes in the numbers of HIV infections (incidence) in some subgroups. (For slide sets of trends among selected populations [2010-2015], see Additional Resources.)

\section{Report Changes}

In response to requests from our partners for expanded data, the 2016 report includes tables that provide stratifications by transmission category and race/ethnicity for multiple years (2011-2016).

- Tables $5 \mathrm{a} / \mathrm{b}$ and $8 \mathrm{a} / \mathrm{b}$ display data on diagnoses of HIV infection and stage 3 (AIDS) classifications, respectively, for infections attributed to male-tomale sexual contact and male-to-male sexual contact and injection drug use, by year and selected characteristics for each racial/ethnic group.
- Data on diagnoses of HIV infection and stage 3 (AIDS) classifications, by year and selected characteristics for each racial/ethnic group, are also presented for infections attributed to injection drug use (Tables $6 \mathrm{a} / \mathrm{b}$ and $9 \mathrm{a} / \mathrm{b}$ ) and heterosexual contact (Tables $7 \mathrm{a} / \mathrm{b}$ and $10 \mathrm{a} / \mathrm{b})$.

Data on survival after a diagnosis of HIV or stage 3 (AIDS) classification are not presented in this report (for survival data, see HIV Supplemental Surveillance Report, Monitoring Selected National HIV Prevention and Care Objectives by Using HIV Surveillance Data-United States and 6 Dependent Areas [Suggested Readings]).

\section{Definitions and Data Specifications}

The term diagnosis of HIV infection is defined as a diagnosis of HIV infection regardless of the stage of disease (stage $0,1,2,3$ [AIDS], or unknown) and refers to all persons with a diagnosis of HIV infection. The term HIV infection, stage 3 (AIDS) and its condensed version - stage 3 (AIDS) — refer specifically to persons with diagnosed HIV whose infection was classified as stage 3 (AIDS) during a given year (for diagnoses) or whose infection has ever been classified as stage 3 (AIDS) (for prevalence and deaths). The 2008 surveillance case definition for HIV infection was used to classify cases diagnosed through 2013. The 2014 revised surveillance case definition was used to classify cases diagnosed after 2013. For more information on HIV surveillance case definitions and the stages of HIV infection, please review the Technical Notes.

The data on diagnoses of HIV infection reflect the date of diagnosis (diagnosed by December 31, 2016; reported to CDC as of June 30,2017), not the date of report to CDC. Similarly, the data specific to stage 3 (AIDS) reflect the first date the criteria for stage 3 (AIDS) were met. HIV diagnoses do not necessarily reflect when a person became infected. HIV surveillance data may not be representative of all persons infected with HIV because not all infected persons have been (1) tested, (2) tested at a time when their infection could be detected and diagnosed, or (3) reported to the surveillance system (e.g., home- 
based testing). Because of reporting delays, the number of cases diagnosed in a given year may be lower than the numbers presented in later reports; however, fluctuations in the number of diagnoses for a calendar year typically subside after 2 to 3 years of reporting.

\section{HighLIGHTS OF ANALYSES}

All rates are per 100,000 population. The standard used for reporting trends in numbers and rates is an increase or a decrease of $5 \%$ or more during the specified time frame (e.g., when comparing 2011 and 2015). Exceptions to this standard were made where numbers were small or rates were based on small numbers. The HIV diagnosis and stage 3 (AIDS) classification data for the year 2016 are preliminary (subject to change) because they are based on only a 6-month reporting delay and therefore should not be included when assessing trends.

Please read all table titles and footnotes carefully to ensure a complete understanding of the displayed data.

\section{Diagnoses of HIV Infection and Diagnoses of Infection Classified as Stage 3 (AIDS)}

\section{Diagnoses of HIV infection}

From 2011 through 2015, the annual number and the rate of diagnoses of HIV infection in the United States decreased (Table 1a). In 2016, the rate was 12.3.

- Age group: From 2011 through 2015, the rate for persons aged 25-29 years increased. The rates for children (aged less than 13 years) and persons aged 13-14, 15-19, 35-39, 40-44, 45$49,50-54,55-59,60-64$ years, and 65 years and older decreased. The rates for persons aged 2024 and 30-34 years remained stable. In 2016, the highest rate was for persons aged 25-29 years (34.8), followed by the rate for persons aged 20-24 years (30.3).

- Race/ethnicity: From 2011 through 2015, the rates for American Indians/Alaska Natives, Asians, and Native Hawaiians/other Pacific Islanders increased. The rates for blacks/African Americans, whites, and persons of multiple races decreased. The rates for Hispanics/Latinos remained stable. In 2016, the highest rate was 43.6 for blacks/African Americans, followed by 17.0 for Hispanics/Latinos, 12.9 for persons of multiple races, 10.2 for American Indians/Alaska
Natives, 8.5 for Native Hawaiians/other Pacific Islanders, 5.5 for Asians, and 5.2 for whites.

- Sex: From 2011 through 2015, the rates for male and female adults and adolescents decreased. In 2016 , males accounted for $81 \%$ of all diagnoses of HIV infection among adults and adolescents. The 2016 rate for male adults and adolescents was 24.3; the 2016 rate for females was 5.4.

- Transmission category: From 2011 through 2015, among male adults and adolescents, the annual number of diagnosed HIV infections attributed to injection drug use, to male-to-male sexual contact and injection drug use, or to heterosexual contact decreased. The number of infections attributed to male-to-male sexual contact remained stable. Among female adults and adolescents, the number of infections attributed to injection drug use or to heterosexual contact decreased. In 2016, among all adults and adolescents, the diagnosed infections attributed to maleto-male sexual contact $(70 \%$, including male-tomale sexual contact and injection drug use) and those attributed to heterosexual contact (24\%) accounted for approximately $94 \%$ of diagnosed HIV infections in the United States.

- Region: From 2011 through 2015, the rates of diagnoses of HIV infection in the Northeast and the South decreased. The rates in the Midwest and the West remained stable. In 2016, rates were 16.8 in the South, 11.2 in the Northeast, 10.2 in the West, and 7.5 in the Midwest.

\section{Stage 3 (AIDS)}

From 2011 through 2015, the annual number and the rate of infections classified as stage 3 (AIDS) in the United States decreased (Table 2a). In 2016, the rate of infections classified as stage 3 (AIDS) was 5.6.

- Age group: Because of the change in case definition in 2014, the trend among children (aged less than 13 years) was not assessed. From 2011 through 2015, the rates for all other age groups decreased. In 2016, the highest rate was for persons aged 35-39 years (11.1), followed by the rate for persons aged 30-34 years (10.9). Please use caution when interpreting data for children (aged less than 13 years): the numbers are small.

- Race/ethnicity: From 2011 through 2015, the rates for all race/ethnicity groups decreased. In 
2016, the highest rate was 21.1 for blacks/African Americans, followed by 9.7 for persons of multiple races, 7.2 for Hispanics/Latinos, 4.3 for American Indians/Alaska Natives, 2.6 for Native Hawaiians/other Pacific Islanders, 2.2 for whites, and 1.9 for Asians.

- Sex: From 2011 through 2015, the rates for male and female adults and adolescents decreased. In 2016 , males accounted for $76 \%$ of all infections classified as stage 3 (AIDS) among adults and adolescents. The 2016 rate for males was 10.5 ; the 2016 rate for females was 3.1.

- Transmission category: From 2011 through 2015 , the annual number of infections classified as stage 3 (AIDS) among male adults and adolescents with HIV infection attributed to male-tomale sexual contact, to injection drug use, to male-to-male sexual contact and injection drug use, or to heterosexual contact decreased. The number of infections classified as stage 3 (AIDS) among female adults and adolescents with HIV infection attributed to injection drug use or to heterosexual contact decreased. Please use caution when interpreting data for children with infection attributed to perinatal transmission: the numbers are small.

- Region: From 2011 through 2015, the rates of infections classified as stage 3 (AIDS) in the 4 regions of the United States decreased. In 2016, rates were 7.8 in the South, 5.5 in the Northeast, 4.1 in the West, and 3.5 in the Midwest.

\section{Deaths}

\section{Deaths of persons with diagnosed HIV infection}

From 2011 through 2015 in the United States, the annual number and rate of deaths of persons with diagnosed HIV infection decreased (Table 16a). In 2015 , the rate of deaths of persons with diagnosed HIV infection was 4.8. Deaths of persons with diagnosed HIV infection may be due to any cause (i.e., may or may not be related to HIV infection).

- Age group: From 2011 through 2015, the rates of deaths for persons aged 60-64 and 65 years and older increased. The rates of deaths for persons aged 20-24, 25-29, 30-34, 35-39, 40-44, 45-49, and 50-54 years decreased. The rate for persons aged 55-59 years remained stable. Please use caution when interpreting data for children (aged less than 13 years at death) and persons aged 13-14 and 15-19 years: the numbers are small.

- Race/ethnicity: From 2011 through 2015, the rates of deaths for persons of multiple races increased. The rates of deaths for Asians, blacks/ African Americans, and Hispanics/Latinos decreased. The rates for American Indians/Alaska Natives and whites remained stable. In 2015, the highest rate of deaths was for blacks/African Americans: 17.5. Please use caution when interpreting data for Native Hawaiians/other Pacific Islanders: the numbers are small.

- Sex and transmission category: From 2011 through 2015, the rates of deaths for male and female adults and adolescents decreased. The number of deaths among males with diagnosed HIV infection attributed to injection drug use, to heterosexual contact, or to perinatal transmission decreased. The numbers of deaths among males attributed to male-to-male sexual contact or maleto-male sexual contact and injection drug use remained stable. The number of deaths among females with infection attributed to injection drug use or to heterosexual contact decreased. The number of deaths among female adults and adolescents with infection attributed to perinatal transmission remained stable. Please use caution when interpreting data for children with infection attributed to perinatal transmission: the numbers are small.

- Region: From 2011 through 2015, the rates of deaths in the Northeast, the Midwest, and the South decreased. The rates of deaths in the West remained stable. In 2015, rates were 6.4 in the Northeast, 6.3 in the South, 3.3 in the West, and 2.6 in the Midwest.

\section{Deaths of persons with infection ever classified as stage 3 (AIDS)}

From 2011 through 2015 in the United States, the annual number and rate of deaths of persons with infection ever classified as stage 3 (AIDS) decreased (Table 17a). In 2015, the rate of deaths of persons with stage 3 (AIDS) was 3.9. Deaths of persons with stage 3 (AIDS) may be due to any cause. 
- Age group: From 2011 through 2015, the rates of deaths among persons aged 60-64 and 65 years and older increased. Rates of deaths among persons aged 20-24, 25-29, 30-34, 35-39, 40-44, 45-49, and 50-54 years decreased. Rates among persons aged 55-59 years remained stable. Please use caution when interpreting data for children (aged less than 13 years) and persons aged 13-14 and 15-19 years: the numbers are small.

- Race/ethnicity: From 2011 through 2015, the rates of deaths for Asians, blacks/African Americans, and Hispanics/Latinos decreased. The rates of deaths for American Indians/Alaska Natives, whites, and persons of multiple races remained stable. In 2015, the highest death rate was for blacks/African Americans: 14.0. Please use caution when interpreting data for Native Hawaiians/other Pacific Islanders: the numbers are small.

- Sex and transmission category: From 2011 through 2015, the rates of deaths for male and female adults and adolescents decreased. The numbers of deaths among males with infection attributed to injection drug use, to heterosexual contact, or to perinatal transmission decreased. The numbers of deaths among males attributed to male-to-male sexual contact or male-to-male sexual contact and injection drug use remained stable. Among female adults and adolescents with infection attributed to injection drug use or to heterosexual contact, the number of deaths decreased. The number of deaths among females with infection attributed to perinatal transmission remained stable. Please use caution when interpreting data for children with infection attributed to perinatal transmission: the numbers are small.

- Region: From 2011 through 2015, the rates of deaths in the Northeast, the Midwest, and the South decreased; the rate in the West remained stable. In 2015, rates were 5.1 in the Northeast, 5.1 in the South, 2.8 in the West, and 2.0 in the Midwest.

\section{Prevalence: Persons Living with Diagnosed HIV Infection and Persons Living with Infection Ever Classified as Stage 3 (AIDS)}

\section{Prevalence of diagnosed HIV infection}

From 2011 through 2015, the number and rate of persons in the United States living with diagnosed HIV infection increased (Table 20a). At year-end 2015, 973,846 persons in the United States were living with diagnosed HIV infection. The prevalence of diagnosed HIV infection was 303.5.

- Age group: At year-end 2015, persons aged 5054 years made up the largest percentage of persons living with diagnosed HIV (18\%). The highest rate (767.9) was among persons aged 50-54 years, followed by those aged 45-49 (703.6) and those aged 55-59 (603.8). From 2011 through 2015 , the largest percentage increase in rates (57\%) was among persons aged 65 years and older (from 94.2 in 2011 to 148.0 in 2015).

- Race/ethnicity: At year-end 2015, the highest rate $(1,017.8)$ and the largest percentage $(42 \%)$ were those for blacks/African Americans. Among the remaining race/ethnicity groups, the rates were 577.3 for persons of multiple races, 379.4 for Hispanics/Latinos, 160.3 for Native Hawaiians/other Pacific Islanders, 150.9 for whites, 122.6 for American Indians/Alaska Natives, and 74.8 for Asians.

- Sex and transmission category: At year-end 2015, the rates were 563.9 for male adults and adolescents, 169.7 for female adults and adolescents, and 4.4 for children (aged less than 13 years at year-end 2015). Among a total of 738,832 males (aged 13 years and older at year-end 2015), $71 \%$ of infections were attributed to male-to-male sexual contact, $10 \%$ to injection drug use, $10 \%$ to heterosexual contact, $7 \%$ to male-to-male sexual contact and injection drug use, and $1 \%$ to perinatal transmission. Among a total of 232,692 females (aged 13 years and older at year-end 2015), $75 \%$ of infections were attributed to heterosexual contact, $22 \%$ to injection drug use, and $2 \%$ to perinatal transmission. Among a total of 2,322 children aged less than 13 years at year-end $2015,81 \%$ of infections were attributed to perinatal transmission. 
- Region: At year-end 2015, the rate was highest in the Northeast at 417.8 , followed by 359.3 in the South, 248.6 in the West, and 170.6 in the Midwest.

\section{Prevalence of stage 3 (AIDS)}

From 2011 through 2015, the number of persons living with infection ever classified as stage 3 (AIDS) increased; the rate remained stable (Table 21a). At year-end 2015, 522,283 persons in the United States were living with infection ever classified as stage 3 (AIDS). The prevalence of infections classified as stage 3 (AIDS) was 162.8.

- Age group: At year-end 2015, the highest rate (479.0) and the largest percentage (20\%) were those for persons aged 50-54 years, followed by persons aged 45-49 (407.4 and 16\%, respectively). From 2011 through 2015, the largest percentage increase in rates $(55 \%)$ was among persons aged 65 years and older (from 63.2 in 2011 to 98.1 in 2015).

- Race/ethnicity: At year-end 2015, the highest rate (539.2) and the largest percentage (41\%) were those for blacks/African Americans. Among the remaining race/ethnicity groups, the rates were 339.9 for persons of multiple races, 214.4 for Hispanics/Latinos, 81.3 for Native Hawaiians/other Pacific Islanders, 78.8 for whites, 63.0 for American Indians/Alaska Natives, and 36.1 for Asians.

- Sex and transmission category: At year-end 2015, the rates were 304.0 for male adults and adolescents, 90.2 for female adults and adolescents, and 0.5 for children (aged less than 13 years at year-end 2015). Among a total of 398,300 males (aged 13 years and older at yearend 2015), 66\% of infections were attributed to male-to-male sexual contact, $13 \%$ to injection drug use, $12 \%$ to heterosexual contact, $8 \%$ to male-to-male sexual contact and injection drug use, and $1 \%$ to perinatal transmission. Among a total of 123,707 females (aged 13 years and older at year-end 2015), 72\% of infections were attributed to heterosexual contact, $25 \%$ to injection drug use, and $2 \%$ to perinatal transmission. Among a total of 276 children aged less than 13 years at year-end 2015, 88\% of infections were attributed to perinatal transmission.
- Region: At year-end 2015, the rate was highest in the Northeast at 238.2, followed by 187.1 in the South, 136.3 in the West, and 86.5 in the Midwest.

\section{AdDITIONAL RESOURCES}

The following were prepared by using HIV surveillance data:

- $M M W R$ articles (selected): http://www.cdc.gov/ hiv/library/reports/mmwr.html

- Other surveillance reports: http://www.cdc.gov/ hiv/library/reports/hiv-surveillance.html

- Public-use slides updated annually: http://www .cdc.gov/hiv/library/slidesets/

- NCHHSTP AtlasPlus [interactive tool for accessing HIV/AIDS, STD, TB, and hepatitis data]: http://www.cdc.gov/nchhstp/atlas/

\section{Suggested READINGS}

CDC. Establishing a holistic framework to reduce inequities in HIV, viral hepatitis, STDs, and tuberculosis in the United States. http://stacks.cdc.gov/ view/cdc/11585. Published October 2010. Accessed October 27, 2017.

CDC. Monitoring selected national HIV prevention and care objectives by using HIV surveillance data-United States and 6 dependent areas, 2015. HIV Surveillance Supplemental Report 2017;22(No. 2). http://www.cdc.gov/hiv/library/ reports/hiv-surveillance.html. Published July 2017. Accessed October 27, 2017.

CDC [Selik RM, Mokotoff ED, Branson B, Owen SM, Whitmore S, Hall HI]. Revised surveillance case definition for HIV infection-United States, 2014. MMWR 2014;63(RR-03):1-10. http://www.cdc .gov/mmwr/indrr_2014.html. Accessed October 27, 2017.

CDC. Revised surveillance case definitions for HIV infection among adults, adolescents, and children aged $<18$ months and for HIV infection and AIDS among children aged 18 months to $<13$ yearsUnited States, 2008. MMWR 2008;57(RR-10):1-12. http://www.cdc.gov/mmwr/indrr_2008.html. Accessed October 27, 2017. 
CDC [Smith DK, Van Handel M, Wolitski RJ, et al]. Vital Signs: Estimated percentages and numbers of adults with indications for preexposure prophylaxis to prevent HIV acquisition-United States, 2015. MMWR 2015;64(46):1291-1295. http:// www.cdc.gov/mmwr/index2015.html. Accessed October 27, 2017.

CDC. Vital Signs: HIV infection, testing, and risk behaviors among youths-United States. MMWR 2012;61(47):971-976. http://www.cdc.gov/mmwr/ index2012.html. Accessed October 27, 2017.

CDC. Vital Signs: HIV testing and diagnosis among adults_-United States. MMWR 2010;59(47): 1550-1555. http://www.cdc.gov/mmwr/ index2010.html. Accessed October 27, 2017.

Cohen SM, Gray KM, Ocfemia MC, Johnson AS, Hall HI. The status of the National HIV Surveillance System, United States, 2013. Public Health Rep 2014;129(4):335-341. doi:10.1177/ 003335491412900408 .

Frieden TR, Foti KE, Mermin J. Applying public health principles to the HIV epidemic - how are we doing? N Engl J Med 2015;373:2281-2287. doi:10.1056/NEJMms 1513641.

Hall HI, Song R, Tang T, et al. HIV trends in the United States: diagnoses and estimated incidence. JMIR Public Health Surveill 2017;3(1):e8. doi.10.2196/publichealth.7051.

Satcher Johnson A, Song R, Hall HI. Estimated HIV incidence, prevalence, and undiagnosed infections in US states and Washington, DC, 2010-2014. J Acquir Immune Defic Syndr 2017;76(2):116-122. doi:10.1097/QAI.0000000000001495.

Song R, Hall HI, Green TA, Szwarcwald CL, Pantazis N. Using CD4 data to estimate HIV incidence, prevalence, and percent of undiagnosed infections in the United States. J Acquir Immune Defic Syndr 2017;74(1):3-9. doi:10.1097/ QAI.0000000000001151. 


\section{SURVEILLANCE OF HIV INFECTION}

This report includes case report data from 50 states, the District of Columbia, and 6 U.S. dependent areas (American Samoa, Guam, the Northern Mariana Islands, Puerto Rico, the Republic of Palau, and the U.S. Virgin Islands) in which laws or regulations require confidential reporting to the jurisdiction (not to CDC), by name, for adults, adolescents, and children with confirmed diagnoses of HIV infection. After the removal of personally identifiable information, data from these reports were submitted to CDC. Although AIDS cases have been reported to CDC since 1981, the date of implementation of HIV infection reporting has differed from jurisdiction to jurisdiction. All states, the District of Columbia, and 6 U.S. dependent areas had fully implemented name-based HIV infection reporting by April 2008.

Please use caution when interpreting data on diagnoses of HIV infection. HIV surveillance reports may not be representative of all persons with HIV because not all infected persons have been (1) tested, (2) tested at a time when the infection could be detected and diagnosed, or (3) reported to the surveillance system (e.g., home-based testing). Also, some states offer anonymous HIV testing; the results of anonymous tests are not reported to the confidential name-based HIV registries of state and local health departments. In addition, testing patterns are influenced by many factors, including the extent to which testing is routinely offered to specific groups and the availability of, and access to, medical care and testing services. The data presented in this report provide minimum counts of persons for whom HIV infection has been diagnosed and reported to the surveillance system. Finally, although all jurisdictions use a uniform case report form, surveillance practices in data collection and updating of case records may differ among jurisdictions.

The completeness of reporting of HIV infection is estimated to be at least $80 \%$ in all but 5 jurisdictions [1].

\section{STAGES OF HIV INFECTION}

Both the 2008 and 2014 HIV case definitions were used to classify HIV infection among adults and adolescents and among children $[2,3]$. In the following lists, some bulleted items are paraphrases, not quotations from the published surveillance case definitions. The intention is to emphasize the differences between the 2008 and 2014 case definitions.

The 2008 case definition was used to classify cases diagnosed through 2013. For adults and adolescents, this definition incorporates an HIV infection staging system that includes AIDS (HIV infection, stage 3 ). The 2008 stages of HIV infection are defined as follows:

- HIV infection, stage 1: No AIDS-defining opportunistic illness (OI) and either CD4+ T-

lymphocyte (CD4 lymphocyte) count of $\geq 500$ cells $/ \mu \mathrm{L}$ or CD4 percentage of total lymphocytes of $\geq 29$.

- HIV infection, stage 2: No AIDS-defining OI and either CD4 lymphocyte count of 200-499 cells/ $\mu \mathrm{L}$ or CD4 percentage of total lymphocytes of 14-28.

- HIV infection, stage 3 (AIDS): Documentation of an AIDS-defining OI or either a CD4 lymphocyte count of $<200$ cells/ $\mu \mathrm{L}$ or CD4 percentage of total lymphocytes of $<14$. Documentation of an AIDS-defining OI supersedes a CD4 lymphocyte count or percentage that would not, by itself, be the basis for a stage 3 (AIDS) classification.

- HIV infection, stage unknown: No reported information on AIDS-defining OIs and no information available on CD4 lymphocyte count or percentage.

The 2014 case definition was used to classify cases diagnosed after 2013. It is similar to the 2008 case definition except for the following: (1) inclusion of criteria for stage $0,(2)$ the inclusion of CD4 lymphocyte testing criteria for stage 3 in children, and (3) changes in the cutoffs for CD4 percentage of total lymphocytes used for classification of stages 1 and 2 in persons aged 6 years and older [3]. The stages of HIV infection in the 2014 case definition are based on age- 
specific CD4 lymphocyte counts or percentages of total lymphocytes and are defined as follows:

- HIV infection, stage 0: Negative (nonreactive) or indeterminate HIV-1 test result within 6 months before the first positive (reactive) HIV-1 test result, or a negative or an indeterminate HIV-1 antibody test result within 6 months before or after the first HIV-1 nucleic acid test result (if the latter was not the first positive HIV test). The stage remains stage 0 until 6 months after the first positive test result. After 6 months, the stage may be reclassified as 1,2,3, or unknown if based on a CD4 lymphocyte test result or the diagnosis of an OI. The diagnosis of an AIDS-defining OI or a low CD4 lymphocyte test result before the 6 months have elapsed does not change the stage from stage 0 to stage 3 .

- HIV infection, stages 1, 2, and 3: Documentation of an AIDS-defining OI (excluding stage 0 as described above) is stage 3. Otherwise, the stage is determined by the lowest CD4 lymphocyte test result $[3, \mathrm{p} 7]$.

- HIV infection, stage unknown: No reported information on AIDS-defining OIs and no information available on CD4 lymphocyte count or percentage.

The transition to CD4 lymphocyte-based criteria for stage 3 (AIDS) among children has resulted in an increase in the number of annual stage 3 (AIDS) classifications among pediatric cases diagnosed after 2013. Before implementation of the 2014 revised case definition, an OI diagnosis was required for a pediatric case to meet the criteria for stage 3 classification.

In this report, the term diagnosis of HIV infection is defined as a diagnosis of HIV infection regardless of the stage of disease (stage $0,1,2,3$ [AIDS], or unknown) and refers to all persons with a diagnosis of HIV infection. The term HIV infection, stage 3 (AIDS) and its condensed version-stage 3 (AIDS) - refer specifically to persons with diagnosed HIV whose infection was classified as stage 3 (AIDS) during a given year (for diagnoses) or whose infection has ever been classified as stage 3 (AIDS) (for prevalence and deaths).

\section{Tabulation and Presentation of Data}

The data in this report include information received by CDC through June 30, 2017. The data are organized into 4 sections.

- Section 1 (Tables $1 \mathrm{a} / \mathrm{b}-15)$ : numbers and rates of diagnoses of HIV infection (any stage) and diagnoses of infection classified as stage 3 (AIDS)

- Section 2 (Tables 16a/b-19a/b): numbers and rates of deaths among persons with diagnosed HIV infection (any stage) and persons with infection ever classified as stage 3 (AIDS)

- Section 3 (Tables 20a/b-23a/b): numbers and rates of persons living with diagnosed HIV infection (any stage) and persons living with infection ever classified as stage 3 (AIDS) (i.e., prevalence)

- Section 4 (Tables 24-29): numbers and rates of diagnoses and prevalence of HIV infection (any stage) and diagnoses and prevalence of infection classified as stage 3 (AIDS), presented by state and metropolitan statistical area (MSA)

Many tables in the 2016 report present data in 2 formats. Tables in the first format — labeled "a" — exclude data from the dependent areas (American Samoa, Guam, the Northern Mariana Islands, Puerto Rico, the Republic of Palau, and the U.S. Virgin Islands). Tables in the second format - labeled " $b$ " — include data from the dependent areas.

Please use caution when interpreting numbers less than 12, and rates and trends based on these numbers.

Numbers of diagnoses for the most recent year (2016) are preliminary (subject to change). Please use caution when interpreting these numbers, and do not include them when assessing trends.

\section{Diagnoses of HIV Infection and of Infection Classified as Stage 3 (AIDS)}

This report is based on diagnoses of HIV infection that the 50 states, the District of Columbia, and 6 U.S. dependent areas reported to CDC as of June 30, 2017. Data from all areas are included in tables displaying numbers and rates of diagnoses of HIV infection and of diagnoses of infection classified as stage 3 (AIDS), by selected characteristics, area of residence, and MSA (Tables 1a/b-15, 24, 25, 28, and 29). For tables presenting diagnosis data, region or area of residence reflects the address at the time of 
diagnosis of HIV infection or at the time of stage 3 (AIDS) classification.

\section{Deaths}

Persons reported to the National HIV Surveillance System are assumed alive unless their deaths have been reported to CDC. Death data include deaths of persons with diagnosed HIV infection (Tables 16a/b and $18 \mathrm{a} / \mathrm{b}$ ) or with infection ever classified as stage 3 (AIDS) (Tables $17 \mathrm{a} / \mathrm{b}$ and $19 \mathrm{a} / \mathrm{b}$ ) regardless of the cause of death. Because of delays in the reporting of deaths, only 5 years (2011-2015) of death data are displayed. The exclusion of data from the most recent year allowed at least 18 months for deaths to be reported to CDC. For the death tables, region or area of residence is based on residence at death. When information on residence at death is not available, the state where a person's death occurred is used.

\section{Prevalence: Persons Living with Diagnosed HIV Infection and Persons Living with Infection Ever Classified as Stage 3 (AIDS)}

Because of delays in the reporting of deaths, only 5 years of prevalence data are displayed. The data reflect persons living with diagnosed HIV infection, regardless of stage of disease, (Tables $20 \mathrm{a} / \mathrm{b}, 22 \mathrm{a} / \mathrm{b}$, 26 , and 28) and persons living with infection ever classified as stage 3 (AIDS) (Tables 21a/b, 23a/b, 27, and 29) at the end of a given year during 2011-2015. The exclusion of data from the most recent year allowed at least 18 months for deaths to be reported and for these deaths to be factored into calculations of prevalence. For tables presenting prevalence data, region or area of residence is based on most recent known address as of the end of the specified year.

\section{Age}

The designation "adults and adolescents" refers to persons aged 13 years and older; the designation "children" refers to persons aged less than 13 years.

For presentations of data on persons living with diagnosed HIV infection (Tables $20 \mathrm{a} / \mathrm{b}, 22 \mathrm{a} / \mathrm{b}$, and 26 ) and presentations of data on persons living with infection classified as stage 3 (AIDS) (Tables $21 \mathrm{a} / \mathrm{b}, 23 \mathrm{a} / \mathrm{b}$, and 27), the age-group assignment (e.g., 20-24 years) or the age designation (e.g., "adults and adolescents") is based on the person's age as of December 31 of the specified year. For presentations of data on deaths of persons with diagnosed HIV infection (Tables 16a/b and $18 \mathrm{a} / \mathrm{b}$ ) and deaths of persons with infection classified as stage 3 (AIDS) (Tables $17 \mathrm{a} / \mathrm{b}$ and $19 \mathrm{a} / \mathrm{b}$ ), agegroup assignment is determined by the person's age at the time of death. For all other tables, the age designation or the specific age-group assignment is based on the person's age at the time of diagnosis (HIV infection or infection classified as stage 3 [AIDS]).

Age-group designations for transmission categories in tables displaying data on prevalence and on deaths are based on a person's age at the end of the specified year or age at death, respectively. The transmission categories among "adults and adolescents" in these tables include persons who were children at the time of diagnosis but who were aged 13 years and older during the specified year or at death.

\section{Sex}

Sex designations in this report are based on a person's sex at birth. Transgender is an umbrella term that is used to identify persons whose sex assigned at birth does not match current gender identity or expression. Data for transgender persons are not presented in this report because information on gender identity (a person's internal understanding of his or her gender or the gender with which a person identifies) is not consistently collected or documented in the data sources used by HIV reporting jurisdictions. HIV surveillance personnel collect data on gender identity, when available, from sources such as case report forms submitted by health care or HIV testing providers and medical records, or by matching with other health department databases (e.g., Ryan White program data). In May 2012, CDC issued guidance to state and local programs on methods for collecting data on transgender persons and working with transgender-specific data. However, characterization of HIV infection among transgender persons may require supplemental data from special studies.

\section{Race and Ethnicity}

In the Federal Register [4] for October 30, 1997, the Office of Management and Budget (OMB) announced the Revisions to the Standards for the Classification of Federal Data on Race and Ethnicity. Implementation by January 1, 2003, was mandated. At a minimum, data on the following race categories should be collected: 
- American Indian or Alaska Native

- Asian

- black or African American

- Native Hawaiian or other Pacific Islander

- white

Additionally, systems must be able to retain information when multiple race categories are reported. In addition to data on race, data on 2 categories of ethnicity should be collected:

- Hispanic or Latino

- not Hispanic or Latino

The Asian or Pacific Islander category displayed in annual surveillance reports published prior to the 2007 surveillance report was split into 2 categories:

(1) Asian and (2) Native Hawaiian or other Pacific Islander. The Asian category (in tables where footnoted) includes the cases in Asians/Pacific Islanders (referred to as legacy cases) that were reported before the implementation of the new race categories in 2003 (e.g., cases of HIV infection that were diagnosed and reported to CDC before 2003 but that were classified as stage 3 [AIDS] after 2003) and a small percentage of cases that were reported after 2003 but that were reported according to the old race category (Asian/ Pacific Islander). In tables of diagnoses of HIV infection during 2011-2016, the Asian category does not include Asian/Pacific Islander cases because these cases were diagnosed after 2003 and were reported to CDC in accordance with OMB's Revisions to the Standards for the Classification of Federal Data on Race and Ethnicity [4].

This report also presents data for persons for whom multiple race categories are reported. In this report, persons categorized by race were not Hispanic or Latino. The number of persons reported in each race category may, however, include persons whose ethnicity was not reported.

\section{Geographic Designations}

Data by region or area of residence reflect the address at the time of diagnosis of HIV infection or at the time of stage 3 (AIDS) classification for tables that present number of diagnoses (Tables $1 \mathrm{a} / \mathrm{b}-15 \mathrm{a} / \mathrm{b}$, $24,25,28,29)$. For tables presenting prevalence data $(20 \mathrm{a} / \mathrm{b}-23 \mathrm{a} / \mathrm{b}, 26-29)$, region or area of residence is based on most recent known address as of the end of the specified year. For the death tables (16a/b-19a/ b), region or area of residence is based on residence at death. When information on residence at death is not available, the state where a person's death occurred is used.

The 4 regions of residence used in this report are defined by the U.S. Census Bureau as follows:

Northeast: Connecticut, Maine, Massachusetts, New Hampshire, New Jersey, New York, Pennsylvania, Rhode Island, and Vermont

Midwest: Illinois, Indiana, Iowa, Kansas, Michigan, Minnesota, Missouri, Nebraska, North Dakota, Ohio, South Dakota, and Wisconsin

South: Alabama, Arkansas, Delaware, District of Columbia, Florida, Georgia, Kentucky, Louisiana, Maryland, Mississippi, North Carolina, Oklahoma, South Carolina, Tennessee, Texas, Virginia, and West Virginia

West: Alaska, Arizona, California, Colorado, Hawaii, Idaho, Montana, Nevada, New Mexico, Oregon, Utah, Washington, and Wyoming

U.S. dependent areas: American Samoa, Guam, the Northern Mariana Islands, Puerto Rico, the Republic of Palau, and the U.S. Virgin Islands

\section{Metropolitan Statistical Areas}

In the Federal Register for June 28, 2010, OMB published revised standards for defining MSAs in federal statistical activities [5]. These standards, which provided for the identification of MSAs in the United States and Puerto Rico, replaced the 2000 standards. The adoption of the new standards was effective as of June 28, 2010. On February 28, 2013, OMB

announced new MSA delineations based on the new standards and Census 2010 data [6]. Tables 28 (data on diagnosed HIV infection) and 29 (data on stage 3 [AIDS]) present numbers and rates of diagnoses and prevalence, by MSA, for areas with populations of 500,000 or more. The MSAs listed in these tables were defined according to OMB's most recent update (February 2013) of statistical areas [6].

\section{Transmission Categories}

Transmission category is the term for the classification of cases that summarizes a person's possible HIV risk factors; the summary classification results from selecting, from the presumed hierarchical order of probability, the 1 (single) risk factor most likely to 
have been responsible for transmission. For surveillance purposes, a diagnosis of HIV infection is counted only once in the hierarchy of transmission categories. Persons with more than 1 reported risk factor for HIV infection are classified in the transmission category listed first in the hierarchy. The exception is men who had sexual contact with other men and injected drugs; this group makes up a separate transmission category.

Persons whose transmission category is classified as male-to-male sexual contact include men who have ever had sexual contact with other men (i.e., homosexual contact) and men who have ever had sexual contact with both men and women (i.e., bisexual contact). Persons whose transmission category is classified as heterosexual contact are persons who have ever had heterosexual contact with a person known to have, or to be at high risk for, HIV infection (e.g., a person who injects drugs). The heterosexual contact category excludes men who have ever had sexual contact with both men and women.

Tables of prevalence data and tables of death data include a separate transmission category of adults and adolescents with HIV infection attributed to perinatal transmission. The category includes persons infected through perinatal transmission but aged 13 years and older during the specified year or at death.

Cases of HIV infection reported without a risk factor listed in the hierarchy of transmission categories are classified as "no identified risk (NIR)." Cases classified as NIR include cases that are being followed up by local health department staff; cases in persons whose risk-factor information is missing because they died, declined to be interviewed, or were lost to follow-up; and cases in persons who were interviewed or for whom other follow-up information was available but for whom no risk factor was identified.

Because a substantial proportion of cases of HIV infection are reported to CDC without an identified risk factor, multiple imputation is used to assign a transmission category [7]. Multiple imputation is a statistical approach in which each missing transmission category is replaced with a set of plausible values that represent the uncertainty about the true, but missing, value [8]. Each resulting data set containing the plausible values is analyzed by using standard procedures, and the results from these analyses are then combined to produce the final results. In tables displaying transmission categories, multiple imputation was used for adults and adolescents, but not for children (because the number of cases in children is small, missing transmission categories were not imputed).

\section{Rates}

Rates per 100,000 population were calculated for (1) the numbers of diagnoses of HIV infection and the numbers of infections classified as stage 3 (AIDS), (2) the numbers of deaths of persons with diagnosed HIV infection and deaths of persons with infection ever classified as stage 3 (AIDS), and

(3) the numbers of persons living with diagnosed HIV infection and persons living with infection ever classified as stage 3 (AIDS).

The population denominators used to compute the rates for the 50 states, the District of Columbia, and Puerto Rico were based on the Vintage 2016 postcensal estimates file (for years 2011-2016) from the U.S. Census Bureau [9]. The population denominators for American Samoa, Guam, the Northern Mariana Islands, the Republic of Palau, and the U.S. Virgin Islands were based on estimates and projections from the U.S. Census Bureau's International Data Base [10]. Each rate was calculated by dividing the total number of diagnoses (or deaths or prevalence) for the calendar year by the population for that calendar year and then multiplying the result by 100,000 . The denominators used for calculating the rates specific to age, sex, and race/ethnicity were computed by applying the appropriate vintage estimates for age, sex, and race/ethnicity for the 50 states and the District of Columbia [9]. The same method was used to calculate the denominators for Puerto Rico, with the exception of race/ethnicity estimates; these data are not available for Puerto Rico (see next paragraph). For the other 5 U.S. dependent areas, estimates from the U.S. Census Bureau's International Data Base were used for ageand sex-specific population denominators [10].

CDC currently does not provide subpopulation rates by race/ethnicity for the 6 U.S. dependent areas because the U.S. Census Bureau does not collect information from all dependent areas. Rates for transmission categories are not provided in this report because of the absence of denominator data from the U.S. Census Bureau, the source of data used for calculating all rates in this report. 


\section{REFERENCES}

1. Karch DL, Chen M, Tang T. Evaluation of the National Human Immunodeficiency Virus Surveillance System for the 2011 diagnosis year. J Public Health Manag Pract 2014;20(6):598-607. doi: 10.1097/ PHH.0000000000000033.

2. CDC. Revised surveillance case definitions for HIV infection among adults, adolescents, and children aged $<18$ months and for HIV infection and AIDS among children aged 18 months to $<13$ years - United States, 2008. MMWR 2008;57(RR-10):1-12.

3. CDC [Selik RM, Mokotoff ED, Branson B, Owen SM, Whitmore S, Hall HI]. Revised surveillance case definition for HIV infection-United States, 2014. MMWR 2014;63(RR-03):1-10.

4. Office of Management and Budget. Revisions to the standards for the classification of federal data on race and ethnicity. Federal Register 1997;62:58782-58790. http://go.usa.gov/vSdR. Accessed October 27, 2017.

5. Office of Management and Budget. 2010 Standards for delineating metropolitan and micropolitan statistical areas. Federal Register 2010;75(123):37246-39052. https://go.usa.gov/xn43u. Accessed October 27, 2017.

6. Office of Management and Budget. Revised delineations of metropolitan statistical areas, new definitions of micropolitan statistical areas and combined statistical areas, and guidance on uses of the delineations of these areas. OMB Bulletin 13-01. https://go.usa.gov/xn43h. Published February 28, 2013. Accessed October 27, 2017.

7. Harrison KM, Kajese T, Hall HI, Song R. Risk factor redistribution of the national HIV/AIDS surveillance data: an alternative approach. Public Health Rep 2008;123(5):618-627.

8. Rubin DB. Multiple Imputation for Nonresponse in Surveys. New York: John Wiley \& Sons Inc; 1987.

9. U.S. Census Bureau. Population and Housing Unit Estimates Datasets. https://go.usa.gov/xn4cc Accessed October 27, 2017.

10. U.S. Census Bureau. International Data Base. http://go.usa.gov/vSUj. Updated August 2017. Accessed October 27, 2017. 
Table 1a. Diagnoses of HIV infection, by year of diagnosis and selected characteristics, 2011-2016-United States

\begin{tabular}{|c|c|c|c|c|c|c|c|c|c|c|c|c|}
\hline & \multicolumn{2}{|c|}{2011} & \multicolumn{2}{|c|}{2012} & \multicolumn{2}{|c|}{2013} & \multicolumn{2}{|c|}{2014} & \multicolumn{2}{|c|}{2015} & \multicolumn{2}{|c|}{2016} \\
\hline & No. & Rate $^{a}$ & No. & Rate $^{a}$ & No. & Rate $^{a}$ & No. & Rate $^{a}$ & No. & Rate $^{a}$ & No. & Rate $^{a}$ \\
\hline \multicolumn{13}{|l|}{ Age at diagnosis (yr) } \\
\hline$<13$ & 198 & 0.4 & 240 & 0.5 & 186 & 0.4 & 180 & 0.3 & 135 & 0.3 & 122 & 0.2 \\
\hline $13-14$ & 42 & 0.5 & 47 & 0.6 & 41 & 0.5 & 35 & 0.4 & 26 & 0.3 & 23 & 0.3 \\
\hline $15-19$ & 1,999 & 9.2 & 1,882 & 8.8 & 1,692 & 8.0 & 1,731 & 8.2 & 1,721 & 8.2 & 1,652 & 7.8 \\
\hline $20-24$ & 7,072 & 31.9 & 7,141 & 31.6 & 7,058 & 30.9 & 7,349 & 32.1 & 7,228 & 31.9 & 6,776 & 30.3 \\
\hline $25-29$ & 6,364 & 29.9 & 6,469 & 30.2 & 6,661 & 30.9 & 7,188 & 32.7 & 7,600 & 33.9 & 7,964 & 34.8 \\
\hline $30-34$ & 5,251 & 25.6 & 5,468 & 26.1 & 5,214 & 24.5 & 5,460 & 25.4 & 5,461 & 25.3 & 5,701 & 26.2 \\
\hline 35-39 & 4,463 & 22.8 & 4,158 & 21.3 & 3,985 & 20.3 & 4,266 & 21.5 & 4,253 & 20.9 & 4,242 & 20.4 \\
\hline 40-44 & 4,791 & 22.8 & 4,449 & 21.1 & 3,945 & 18.9 & 3,789 & 18.4 & 3,416 & 16.9 & 3,334 & 16.9 \\
\hline $45-49$ & 4,573 & 20.6 & 4,302 & 19.8 & 3,934 & 18.5 & 3,627 & 17.4 & 3,319 & 16.0 & 3,156 & 15.1 \\
\hline 50-54 & 3,350 & 14.8 & 3,199 & 14.2 & 2,982 & 13.2 & 2,907 & 12.9 & 2,987 & 13.4 & 2,959 & 13.5 \\
\hline $55-59$ & 1,990 & 9.8 & 1,928 & 9.3 & 2,022 & 9.5 & 1,938 & 9.0 & 1,886 & 8.7 & 1,923 & 8.7 \\
\hline 60-64 & 1,068 & 6.0 & 1,059 & 5.9 & 1,074 & 5.9 & 975 & 5.3 & 997 & 5.2 & 1,089 & 5.6 \\
\hline$\geq 65$ & 823 & 2.0 & 826 & 1.9 & 858 & 1.9 & 831 & 1.8 & 847 & 1.8 & 841 & 1.7 \\
\hline \multicolumn{13}{|l|}{ Race/ethnicity } \\
\hline American Indian/Alaska Native & 143 & 6.2 & 176 & 7.6 & 154 & 6.6 & 187 & 8.0 & 197 & 8.3 & 243 & 10.2 \\
\hline Asian & 744 & 4.9 & 784 & 5.0 & 810 & 5.0 & 938 & 5.6 & 947 & 5.5 & 977 & 5.5 \\
\hline Black/African American & 18,992 & 49.5 & 18,258 & 47.1 & 17,470 & 44.6 & 17,530 & 44.4 & 17,432 & 43.7 & 17,528 & 43.6 \\
\hline Hispanic/Latino ${ }^{b}$ & 9,292 & 17.9 & 9,291 & 17.5 & 9,097 & 16.8 & 9,710 & 17.6 & 9,695 & 17.2 & 9,766 & 17.0 \\
\hline Native Hawaiian/Other Pacific Islander & 55 & 10.8 & 54 & 10.4 & 49 & 9.2 & 44 & 8.1 & 80 & 14.4 & 48 & 8.5 \\
\hline White & 11,146 & 5.6 & 11,039 & 5.6 & 10,636 & 5.4 & 10,618 & 5.4 & 10,465 & 5.3 & 10,345 & 5.2 \\
\hline Multiple races & 1,612 & 27.7 & 1,566 & 26.1 & 1,436 & 23.2 & 1,249 & 19.6 & 1,060 & 16.1 & 875 & 12.9 \\
\hline \multicolumn{13}{|l|}{$\begin{array}{l}\text { Transmission category }{ }^{\mathrm{c}} \\
\text { Male adult or adolescent }\end{array}$} \\
\hline Male-to-male sexual contact & 26,052 & - & 26,254 & - & 25,719 & - & 26,642 & - & 26,459 & - & 26,570 & - \\
\hline Injection drug use & 1,666 & - & 1,489 & - & 1,396 & - & 1,297 & - & 1,343 & - & 1,285 & - \\
\hline $\begin{array}{l}\text { Male-to-male sexual contact and } \\
\text { injection drug use }\end{array}$ & 1,455 & - & 1,407 & - & 1,288 & - & 1,271 & - & 1,270 & - & 1,201 & - \\
\hline Heterosexual contact ${ }^{d}$ & 3,745 & - & 3,461 & - & 3,323 & - & 3,223 & - & 3,187 & - & 3,037 & - \\
\hline Othere ${ }^{e}$ & 46 & - & 59 & - & 46 & - & 44 & - & 46 & - & 38 & - \\
\hline Subtotal & 32,964 & 26.1 & 32,671 & 25.6 & 31,772 & 24.7 & 32,476 & 25.0 & 32,306 & 24.7 & 32,131 & 24.3 \\
\hline \multicolumn{13}{|l|}{ Female adult or adolescent } \\
\hline Injection drug use & 1,262 & - & 1,147 & - & 1,002 & - & 933 & - & 1,004 & - & 939 & - \\
\hline Heterosexual contact ${ }^{d}$ & 7,517 & - & 7,071 & - & 6,640 & - & 6,653 & - & 6,401 & - & 6,541 & - \\
\hline Othere & 43 & - & 40 & - & 52 & - & 35 & - & 30 & - & 49 & - \\
\hline Subtotal & 8,822 & 6.7 & 8,257 & 6.2 & 7,694 & 5.7 & 7,620 & 5.6 & 7,435 & 5.4 & 7,529 & 5.4 \\
\hline \multicolumn{13}{|l|}{ Child ( $<13$ yrs at diagnosis) } \\
\hline Perinatal & 146 & - & 173 & - & 122 & - & 137 & - & 100 & - & 99 & - \\
\hline Other ${ }^{f}$ & 52 & - & 67 & - & 64 & - & 43 & - & 35 & - & 23 & - \\
\hline Subtotal & 198 & 0.4 & 240 & 0.5 & 186 & 0.4 & 180 & 0.3 & 135 & 0.3 & 122 & 0.2 \\
\hline \multicolumn{13}{|l|}{ Region of residenceg } \\
\hline Northeast & 7,755 & 13.9 & 7,551 & 13.5 & 7,013 & 12.5 & 6,994 & 12.5 & 6,502 & 11.6 & 6,309 & 11.2 \\
\hline Midwest & 5,391 & 8.0 & 5,484 & 8.1 & 5,327 & 7.9 & 5,106 & 7.5 & 5,224 & 7.7 & 5,068 & 7.5 \\
\hline South & 21,291 & 18.3 & 20,493 & 17.5 & 20,020 & 16.9 & 20,345 & 17.0 & 20,442 & 16.9 & 20,588 & 16.8 \\
\hline West & 7,547 & 10.4 & 7,640 & 10.4 & 7,292 & 9.8 & 7,831 & 10.4 & 7,708 & 10.2 & 7,817 & 10.2 \\
\hline Total & 41,984 & 13.5 & 41,168 & 13.1 & 39,652 & 12.5 & 40,276 & 12.6 & 39,876 & 12.4 & 39,782 & 12.3 \\
\hline
\end{tabular}

Note. Data for the year 2016 are preliminary (subject to change) because they are based on only a 6-month reporting delay. Data for the year 2016 should not be used when assessing trends.

a Rates are per 100,000 population. Rates are not calculated by transmission category because of the lack of denominator data.

$\mathrm{b}_{\text {Hispanics/Latinos can be of any race. }}$

${ }^{\mathrm{C}}$ Data have been statistically adjusted to account for missing transmission category; therefore, values may not sum to column subtotals and total.

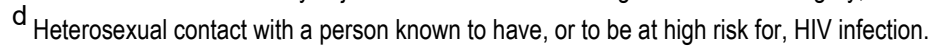

e Includes hemophilia, blood transfusion, perinatal exposure, and risk factor not reported or not identified.

f Includes hemophilia, blood transfusion, and risk factor not reported or not identified.

$\mathrm{g}$ Data are based on residence at time of diagnosis of HIV infection. 
Table 1b. Diagnoses of HIV infection, by year of diagnosis and selected characteristics, 2011-2016-United States and 6 dependent areas




Table 2a. Stage 3 (AIDS), by year of diagnosis and selected characteristics, 2011-2016 and cumulative-United States

\begin{tabular}{|c|c|c|c|c|c|c|c|c|c|c|c|c|c|}
\hline & \multicolumn{2}{|c|}{2011} & \multicolumn{2}{|c|}{2012} & \multicolumn{2}{|c|}{2013} & \multicolumn{2}{|c|}{2014} & \multicolumn{2}{|c|}{2015} & \multicolumn{2}{|c|}{2016} & \multirow{2}{*}{$\begin{array}{c}\text { Cumulative }^{\mathrm{a}} \\
\text { No. }\end{array}$} \\
\hline & No. & Rate $^{b}$ & No. & Rate $^{b}$ & No. & Rate $^{b}$ & No. & Rate $^{b}$ & No. & Rate $^{b}$ & No. & Rate $^{b}$ & \\
\hline \multicolumn{14}{|l|}{ Age at diagnosis (yr) } \\
\hline$<13^{\mathrm{c}}$ & 18 & 0.0 & 11 & 0.0 & 9 & 0.0 & 71 & 0.1 & 40 & 0.1 & 38 & 0.1 & 9,573 \\
\hline $13-14$ & 41 & 0.5 & 28 & 0.3 & 28 & 0.3 & 15 & 0.2 & 9 & 0.1 & 12 & 0.1 & 1,478 \\
\hline $15-19$ & 434 & 2.0 & 356 & 1.7 & 401 & 1.9 & 188 & 0.9 & 191 & 0.9 & 209 & 1.0 & 9,298 \\
\hline $20-24$ & 1,986 & 9.0 & 1,914 & 8.5 & 2,009 & 8.8 & 1,351 & 5.9 & 1,295 & 5.7 & 1,252 & 5.6 & 53,874 \\
\hline $25-29$ & 2,799 & 13.1 & 2,776 & 13.0 & 2,788 & 12.9 & 2,341 & 10.7 & 2,370 & 10.6 & 2,426 & 10.6 & 145,223 \\
\hline $30-34$ & 3,164 & 15.4 & 3,258 & 15.6 & 2,927 & 13.8 & 2,407 & 11.2 & 2,412 & 11.2 & 2,384 & 10.9 & 228,528 \\
\hline $35-39$ & 3,226 & 16.5 & 2,961 & 15.2 & 2,826 & 14.4 & 2,404 & 12.1 & 2,241 & 11.0 & 2,308 & 11.1 & 246,794 \\
\hline $40-44$ & 3,810 & 18.1 & 3,478 & 16.5 & 3,086 & 14.8 & 2,482 & 12.1 & 2,209 & 11.0 & 2,049 & 10.4 & 207,611 \\
\hline $45-49$ & 3,987 & 18.0 & 3,686 & 17.0 & 3,324 & 15.7 & 2,677 & 12.8 & 2,411 & 11.6 & 2,152 & 10.3 & 143,912 \\
\hline $50-54$ & 2,905 & 12.9 & 2,906 & 12.9 & 2,780 & 12.3 & 2,300 & 10.2 & 2,263 & 10.2 & 2,165 & 9.9 & 87,792 \\
\hline $55-59$ & 1,814 & 9.0 & 1,786 & 8.6 & 1,786 & 8.4 & 1,585 & 7.4 & 1,480 & 6.8 & 1,499 & 6.8 & 49,416 \\
\hline $60-64$ & 915 & 5.1 & 1,011 & 5.7 & 1,031 & 5.7 & 850 & 4.6 & 837 & 4.4 & 913 & 4.7 & 26,487 \\
\hline$\geq 65$ & 730 & 1.8 & 729 & 1.7 & 763 & 1.7 & 738 & 1.6 & 722 & 1.5 & 753 & 1.5 & 22,360 \\
\hline \multicolumn{14}{|l|}{ Race/ethnicity } \\
\hline American Indian/Alaska Native & 99 & 4.3 & 91 & 3.9 & 85 & 3.6 & 86 & 3.7 & 93 & 3.9 & 102 & 4.3 & 3,580 \\
\hline Asiand & 348 & 2.3 & 355 & 2.3 & 345 & 2.1 & 322 & 1.9 & 320 & 1.9 & 335 & 1.9 & 10,067 \\
\hline Black/African American & 12,269 & 32.0 & 11,965 & 30.9 & 11,214 & 28.7 & 9,071 & 23.0 & 8,623 & 21.6 & 8,501 & 21.1 & 508,711 \\
\hline Hispanic/Latino ${ }^{\mathrm{e}}$ & 5,483 & 10.6 & 5,247 & 9.9 & 5,102 & 9.4 & 4,228 & 7.7 & 4,061 & 7.2 & 4,111 & 7.2 & 231,473 \\
\hline $\begin{array}{l}\text { Native Hawaiian/ } \\
\text { Other Pacific Islander }\end{array}$ & 30 & 5.9 & 24 & 4.6 & 22 & 4.1 & 17 & 3.1 & 24 & 4.3 & 15 & 2.6 & 837 \\
\hline White & 6,379 & 3.2 & 6,092 & 3.1 & 5,858 & 3.0 & 4,826 & 2.4 & 4,629 & 2.3 & 4,442 & 2.2 & 439,998 \\
\hline Multiple races & 1,221 & 21.0 & 1,126 & 18.7 & 1,132 & 18.3 & 859 & 13.5 & 730 & 11.1 & 654 & 9.7 & 37,588 \\
\hline \multicolumn{14}{|l|}{$\begin{array}{l}\text { Transmission category } \\
\text { Male adult or adolescent }\end{array}$} \\
\hline Male-to-male sexual contact & 13,493 & - & 13,212 & - & 12,997 & - & 10,413 & - & 10,067 & - & 10,075 & - & 599,230 \\
\hline Injection drug use & 1,657 & - & 1,557 & - & 1,348 & - & 1,159 & - & 1,010 & - & 952 & - & 185,414 \\
\hline $\begin{array}{l}\text { Male-to-male sexual contact } \\
\text { and injection drug use }\end{array}$ & 1,256 & - & 1,185 & - & 1,006 & - & 782 & - & 786 & - & 751 & - & 87,872 \\
\hline Heterosexual contact ${ }^{9}$ & 2,822 & - & 2,666 & - & 2,550 & - & 2,157 & - & 1,989 & - & 1,992 & - & 86,911 \\
\hline Other ${ }^{h}$ & 123 & - & 109 & - & 127 & - & 80 & - & 99 & - & 80 & - & 11,694 \\
\hline Subtotal & 19,350 & 15.3 & 18,729 & 14.7 & 18,028 & 14.0 & 14,591 & 11.2 & 13,951 & 10.6 & 13,851 & 10.5 & 971,120 \\
\hline \multicolumn{14}{|l|}{ Female adult or adolescent } \\
\hline Injection drug use & 1,285 & - & 1,176 & - & 1,023 & - & 840 & - & 786 & - & 728 & - & 91,021 \\
\hline Heterosexual contact ${ }^{g}$ & 5,049 & - & 4,882 & - & 4,560 & - & 3,820 & - & 3,625 & - & 3,434 & - & 154,584 \\
\hline Other ${ }^{h}$ & 127 & - & 103 & - & 139 & - & 87 & - & 78 & - & 109 & - & 6,048 \\
\hline Subtotal & 6,461 & 4.9 & 6,160 & 4.6 & 5,721 & 4.2 & 4,747 & 3.5 & 4,489 & 3.3 & 4,271 & 3.1 & 251,653 \\
\hline \multicolumn{14}{|l|}{ Child (<13 yrs at diagnosis) } \\
\hline Perinatal & 14 & - & 9 & - & 8 & - & 61 & - & 31 & - & 31 & - & 8,697 \\
\hline Other $^{i}$ & 4 & - & 2 & - & 1 & - & 10 & - & 9 & - & 7 & - & 876 \\
\hline Subtotal & 18 & 0.0 & 11 & 0.0 & 9 & 0.0 & 71 & 0.1 & 40 & 0.1 & 38 & 0.1 & 9,573 \\
\hline \multicolumn{14}{|l|}{ Region of residence } \\
\hline Northeast & 5,106 & 9.2 & 4,744 & 8.5 & 4,329 & 7.7 & 3,508 & 6.3 & 3,303 & 5.9 & 3,088 & 5.5 & 354,229 \\
\hline Midwest & 3,250 & 4.8 & 3,115 & 4.6 & 2,957 & 4.4 & 2,476 & 3.7 & 2,329 & 3.4 & 2,359 & 3.5 & 132,116 \\
\hline South & 13,163 & 11.3 & 12,847 & 11.0 & 12,544 & 10.6 & 10,134 & 8.5 & 9,742 & 8.0 & 9,584 & 7.8 & 501,523 \\
\hline West & 4,310 & 5.9 & 4,194 & 5.7 & 3,928 & 5.3 & 3,291 & 4.4 & 3,106 & 4.1 & 3,129 & 4.1 & 244,478 \\
\hline Total $^{k}$ & 25,829 & 8.3 & 24,900 & 7.9 & 23,758 & 7.5 & 19,409 & 6.1 & 18,480 & 5.8 & 18,160 & 5.6 & $1,232,346$ \\
\hline $\begin{array}{l}\text { Note. Data for the year } 2016 \text { are pr } \\
\text { Numbers less than } 12 \text {, and rates ar }\end{array}$ & $\begin{array}{l}\text { iminary (su } \\
\text { l trends bas }\end{array}$ & $\begin{array}{l}\text { bject to } \\
\text { eed on tl }\end{array}$ & e) beca & ey & sed on & a $6-m$ & reporting & lay. D & or the ye & 016 & not be & whe & ssing trends. \\
\hline$a_{\text {From the beginning of the epiden }}$ & c through 2 & 016. & & & & & & & & & & & \\
\hline${ }^{b}$ Rates are per 100,000 population & Rates are & not calcul & $d$ by trans & hission ca & gory becau & e of the & of denomi & nator da & & & & & \\
\hline${ }^{\mathrm{c}}$ The criteria for stage 3 (AIDS) cla & sification a & mong pec & ric cases $\mathrm{V}$ & ere expar & d in 2014 & see Tech & al Notes). & & & & & & \\
\hline $\mathrm{d}_{\text {Includes Asian/Pacific Islander le }}$ & acy cases & see Tech & al Notes). & & & & & & & & & & \\
\hline $\mathrm{e}_{\text {Hispanics/Latinos can be of any } r}$ & & & & & & & & & & & & & \\
\hline${ }^{\mathrm{f}}$ Data have been statistically adjus & to acco & nt for mis & transmis & sion categ & therefore & values & not sum to & column & otals ar & & & & \\
\hline$g_{\text {Heterosexual contact with a pers }}$ & known to & have, or $\mathrm{t}$ & e at high $r$ & sk for, HI & fection. & & & & & & & & \\
\hline $\mathrm{h}_{\text {Includes hemophilia, blood transf }}$ & sion, perinz & tal expos & , and risk & actor not & rted or 1 & denti & & & & & & & \\
\hline I Includes hemophilia, blood transf & sion, and ri & sk factor & reported o & not iden & & & & & & & & & \\
\hline $\mathrm{j}$ Data are based on residence at ti & e of stage & 3 (AIDS) & ssification. & & & & & & & & & & \\
\hline $\mathrm{k}$ Cumulative total includes $92 \mathrm{pe}$ & swhos & celeth & sunkng & & & & & & & & & & \\
\hline
\end{tabular}


Table 2b. Stage 3 (AIDS), by year of diagnosis and selected characteristics, 2011-2016 and cumulative-United States and 6 dependent areas

\begin{tabular}{|c|c|c|c|c|c|c|c|c|c|c|c|c|c|}
\hline & \multicolumn{2}{|c|}{2011} & \multicolumn{2}{|c|}{2012} & \multicolumn{2}{|c|}{2013} & \multicolumn{2}{|c|}{2014} & \multicolumn{2}{|c|}{2015} & \multicolumn{2}{|c|}{2016} & \multirow{2}{*}{$\begin{array}{c}\text { Cumulative }^{a} \\
\text { No. }\end{array}$} \\
\hline & No. & Rate $^{b}$ & No. & Rate $^{b}$ & No. & Rate $^{b}$ & No. & Rate $^{b}$ & No. & Rate $^{b}$ & No. & Rate $^{b}$ & \\
\hline \multicolumn{14}{|l|}{ Age at diagnosis (yr) } \\
\hline$<13^{c}$ & 19 & 0.0 & 11 & 0.0 & 9 & 0.0 & 71 & 0.1 & 40 & 0.1 & 39 & 0.1 & 10,000 \\
\hline $13-14$ & 43 & 0.5 & 29 & 0.3 & 29 & 0.3 & 15 & 0.2 & 9 & 0.1 & 12 & 0.1 & 1,538 \\
\hline 15-19 & 442 & 2.0 & 357 & 1.6 & 406 & 1.9 & 193 & 0.9 & 191 & 0.9 & 212 & 1.0 & 9,542 \\
\hline 20-24 & 2,002 & 8.9 & 1,933 & 8.4 & 2,031 & 8.8 & 1,371 & 5.9 & 1,307 & 5.7 & 1,266 & 5.6 & 55,326 \\
\hline $25-29$ & 2,833 & 13.1 & 2,809 & 13.0 & 2,816 & 12.9 & 2,360 & 10.6 & 2,392 & 10.6 & 2,438 & 10.5 & 149,471 \\
\hline 30-34 & 3,210 & 15.4 & 3,299 & 15.6 & 2,973 & 13.8 & 2,446 & 11.2 & 2,437 & 11.2 & 2,405 & 10.9 & 235,471 \\
\hline $35-39$ & 3,298 & 16.6 & 3,017 & 15.3 & 2,878 & 14.5 & 2,453 & 12.2 & 2,273 & 11.1 & 2,347 & 11.2 & 254,080 \\
\hline $40-44$ & 3,881 & 18.2 & 3,551 & 16.7 & 3,144 & 14.9 & 2,538 & 12.2 & 2,243 & 11.0 & 2,077 & 10.4 & 213,477 \\
\hline $45-49$ & 4,081 & 18.2 & 3,767 & 17.1 & 3,395 & 15.8 & 2,730 & 12.9 & 2,450 & 11.6 & 2,194 & 10.4 & 147,932 \\
\hline $50-54$ & 2,985 & 13.1 & 2,965 & 13.0 & 2,846 & 12.5 & 2,347 & 10.3 & 2,308 & 10.2 & 2,197 & 9.9 & 90,220 \\
\hline $55-59$ & 1,846 & 9.0 & 1,825 & 8.7 & 1,834 & 8.6 & 1,623 & 7.5 & 1,503 & 6.8 & 1,529 & 6.9 & 50,882 \\
\hline $60-64$ & 932 & 5.2 & 1,032 & 5.7 & 1,057 & 5.8 & 872 & 4.6 & 845 & 4.4 & 926 & 4.7 & 27,308 \\
\hline$\geq 65$ & 761 & 1.8 & 760 & 1.7 & 785 & 1.7 & 754 & 1.6 & 740 & 1.5 & 767 & 1.5 & 23,348 \\
\hline \multicolumn{14}{|l|}{ Race/ethnicity } \\
\hline American Indian/Alaska Native & 99 & - & 91 & - & 85 & - & 86 & - & 93 & - & 102 & - & 3,581 \\
\hline Asian ${ }^{d}$ & 350 & - & 355 & - & 345 & - & 323 & - & 321 & - & 336 & - & 10,124 \\
\hline Black/African American & 12,275 & - & 11,975 & - & 11,219 & - & 9,079 & - & 8,632 & - & 8,504 & - & 509,200 \\
\hline Hispanic/Latino ${ }^{\mathrm{e}}$ & 5,976 & - & 5,689 & - & 5,541 & - & 4,581 & - & 4,305 & - & 4,350 & - & 267,013 \\
\hline $\begin{array}{l}\text { Native Hawaiian/ } \\
\text { Other Pacific Islander }\end{array}$ & 31 & - & 25 & - & 22 & - & 17 & - & 26 & - & 20 & - & 864 \\
\hline White & 6,379 & - & 6,094 & - & 5,859 & - & 4,828 & - & 4,631 & - & 4,443 & - & 440,117 \\
\hline Multiple races & 1,223 & - & 1,126 & - & 1,132 & - & 859 & - & 730 & - & 654 & - & 37,604 \\
\hline \multicolumn{14}{|l|}{$\begin{array}{l}\text { Transmission category } \\
\text { Male adult or adolescent }\end{array}$} \\
\hline Male-to-male sexual contact & 13,612 & - & 13,332 & - & 13,134 & - & 10,513 & - & 10,155 & - & 10,157 & - & 605,555 \\
\hline Injection drug use & 1,759 & - & 1,652 & - & 1,424 & - & 1,229 & - & 1,057 & - & 993 & - & 198,978 \\
\hline $\begin{array}{l}\text { Male-to-male sexual contact } \\
\text { and injection drug use }\end{array}$ & 1,279 & - & 1,206 & - & 1,018 & - & 791 & - & 800 & - & 769 & - & 90,494 \\
\hline Heterosexual contact ${ }^{9}$ & 2,928 & - & 2,743 & - & 2,638 & - & 2,232 & - & 2,038 & - & 2,045 & - & 91,070 \\
\hline Other ${ }^{h}$ & 126 & - & 112 & - & 129 & - & 81 & - & 101 & - & 82 & - & 11,900 \\
\hline Subtotal & 19,704 & 15.4 & 19,045 & 14.8 & 18,344 & 14.1 & 14,845 & 11.3 & 14,150 & 10.7 & 14,047 & 10.5 & 997,997 \\
\hline \multicolumn{14}{|l|}{ Female adult or adolescent } \\
\hline Injection drug use & 1,311 & - & 1,200 & - & 1,051 & - & 869 & - & 794 & - & 736 & - & 94,118 \\
\hline Heterosexual contact ${ }^{9}$ & 5,168 & - & 4,994 & - & 4,659 & - & 3,896 & - & 3,675 & - & 3,478 & - & 160,285 \\
\hline Other ${ }^{h}$ & 131 & - & 105 & - & 141 & - & 92 & - & 79 & - & 109 & - & 6,195 \\
\hline Subtotal & 6,610 & 4.9 & 6,299 & 4.7 & 5,850 & 4.3 & 4,857 & 3.5 & 4,548 & 3.3 & 4,323 & 3.1 & 260,598 \\
\hline \multicolumn{14}{|l|}{ Child (<13 yrs at diagnosis) } \\
\hline Perinatal & 15 & - & 9 & - & 8 & - & 61 & - & 31 & - & 32 & - & 9,099 \\
\hline Other $^{i}$ & 4 & - & 2 & - & 1 & - & 10 & - & 9 & - & 7 & - & 901 \\
\hline Subtotal & 19 & 0.0 & 11 & 0.0 & 9 & 0.0 & 71 & 0.1 & 40 & 0.1 & 39 & 0.1 & 10,000 \\
\hline \multicolumn{14}{|l|}{ Region of residence } \\
\hline Northeast & 5,106 & 9.2 & 4,744 & 8.5 & 4,329 & 7.7 & 3,508 & 6.3 & 3,303 & 5.9 & 3,088 & 5.5 & 354,229 \\
\hline Midwest & 3,250 & 4.8 & 3,115 & 4.6 & 2,957 & 4.4 & 2,476 & 3.7 & 2,329 & 3.4 & 2,359 & 3.5 & 132,116 \\
\hline South & 13,163 & 11.3 & 12,847 & 11.0 & 12,544 & 10.6 & 10,134 & 8.5 & 9,742 & 8.0 & 9,584 & 7.8 & 501,523 \\
\hline West & 4,310 & 5.9 & 4,194 & 5.7 & 3,928 & 5.3 & 3,291 & 4.4 & 3,106 & 4.1 & 3,129 & 4.1 & 244,478 \\
\hline U.S. dependent areas & 504 & 12.4 & 455 & 11.3 & 445 & 11.2 & 364 & 9.3 & 258 & 6.7 & 249 & 6.5 & 36,249 \\
\hline Total $^{k}$ & 26,333 & 8.3 & 25,355 & 8.0 & 24,203 & 7.6 & 19,773 & 6.1 & 18,738 & 5.8 & 18,409 & 5.6 & $1,268,595$ \\
\hline $\begin{array}{l}\text { Note. Data for the year } 2016 \text { are pr } \\
\text { Numbers less than } 12 \text {, and rates a }\end{array}$ & iminary (s) & ect to & e) becau & they & $\begin{array}{l}\text { sed on } \\
\text { rpreted }\end{array}$ & a 6-m & eporti & ay. D & for the $y$ &  & not be & whe & essing trends \\
\hline$a$ From the beginning of the epidem & $c$ through 2 & 016. & & & & & & & & & & & \\
\hline $\begin{array}{l}\mathrm{b} \text { Rates are per } 100,000 \text { population } \\
\text { category because of the lack of } d\end{array}$ & $\begin{array}{l}\text { Rates by ra } \\
\text { nominator } \mathrm{C}\end{array}$ & $\begin{array}{l}\text { ace/ethn } \\
\text { data. }\end{array}$ & re not $p$ & ded bec & U.S. C & us info & in for & teper & reas is & ted. & re not & dlatec & ansmission \\
\hline${ }^{\mathrm{C}}$ The criteria for stage 3 (AIDS) cla & sification ar & mong ped & ric cases $n$ & ere expar & in 2014 & see Tech & I Notes). & & & & & & \\
\hline $\mathrm{d}_{\text {Includes Asian/Pacific Islander leg }}$ & acy cases & see Techr & I Notes). & & & & & & & & & & \\
\hline${ }^{e}{ }_{\text {Hispanics/Latinos can be of any } r a}$ & & & & & & & & & & & & & \\
\hline${ }^{f}$ Data have been statistically adjust & ed to accou & nt for mis & transmis & sion categ & therefor & values & lot sum & olumn & totals an & & & & \\
\hline$g_{\text {Heterosexual contact with a perso }}$ & known to & have, or t & e at high $r$ & sk for, HI & fection. & & & & & & & & \\
\hline${ }^{\mathrm{h}}$ Includes hemophilia, blood transfu & sion, perina & tal expos & , and risk & actor not & orted or $n$ & identifi & & & & & & & \\
\hline i Includes hemophilia, blood transfu & sion, and ris & sk factor $n$ & reported o & not iden & & & & & & & & & \\
\hline $\mathrm{j}$ Data are based on residence at tir & e of stage & 3 (AIDS) & issification. & & & & & & & & & & \\
\hline umulative total includes 92 pers & ns whose $\mathrm{r}$ & ace/ethni & is unkn & & & & & & & & & & \\
\hline
\end{tabular}




\begin{tabular}{|c|c|c|c|c|c|c|c|c|c|c|c|c|c|c|c|c|}
\hline & \multicolumn{2}{|c|}{$\begin{array}{c}\text { American } \\
\text { Indian/ } \\
\text { Alaska Native }\end{array}$} & \multicolumn{2}{|c|}{ Asian } & \multicolumn{2}{|c|}{$\begin{array}{l}\text { Black/African } \\
\text { American }\end{array}$} & \multicolumn{2}{|c|}{$\begin{array}{l}\text { Hispanicl } \\
\text { Latino }^{a}\end{array}$} & \multicolumn{2}{|c|}{$\begin{array}{c}\text { Native } \\
\text { Hawaiian/ } \\
\text { Other Pacific } \\
\text { Islander }\end{array}$} & \multicolumn{2}{|c|}{ White } & \multicolumn{2}{|c|}{ Multiple races } & \multicolumn{2}{|c|}{ Total } \\
\hline & No. & Rate $^{b}$ & No. & Rate $^{b}$ & No. & Rate $^{b}$ & No. & Rate $^{b}$ & No. & Rate $^{b}$ & No. & Rate $^{b}$ & No. & Rate $^{b}$ & No. & Rate $^{b}$ \\
\hline \multicolumn{17}{|l|}{ Age at diagnosis (yr) } \\
\hline$<13$ & 0 & 0.0 & 8 & 0.3 & 78 & 1.1 & 16 & 0.1 & 0 & 0.0 & 16 & 0.1 & 4 & 0.2 & 122 & 0.2 \\
\hline $13-14$ & 0 & 0.0 & 3 & 0.7 & 15 & 1.3 & 3 & 0.2 & 0 & 0.0 & 0 & 0.0 & 2 & 0.7 & 23 & 0.3 \\
\hline $15-19$ & 3 & 1.6 & 19 & 1.8 & 1,029 & 34.5 & 334 & 7.0 & 2 & 4.9 & 222 & 2.0 & 43 & 6.1 & 1,652 & 7.8 \\
\hline 20-24 & 36 & 18.6 & 128 & 10.1 & 3,584 & 108.7 & 1,623 & 33.8 & 3 & 6.7 & 1,213 & 10.0 & 189 & 30.5 & 6,776 & 30.3 \\
\hline $25-29$ & 61 & 32.0 & 197 & 13.0 & 3,661 & 112.4 & 2,085 & 45.2 & 10 & 19.2 & 1,773 & 13.9 & 177 & 35.9 & 7,964 & 34.8 \\
\hline $30-34$ & 42 & 25.5 & 175 & 11.4 & 2,201 & 78.6 & 1,689 & 37.9 & 9 & 18.1 & 1,465 & 11.8 & 120 & 30.5 & 5,701 & 26.2 \\
\hline $35-39$ & 24 & 15.9 & 129 & 9.0 & 1,680 & 63.0 & 1,205 & 28.1 & 8 & 18.3 & 1,108 & 9.4 & 88 & 26.0 & 4,242 & 20.4 \\
\hline $40-44$ & 28 & 19.9 & 113 & 8.0 & 1,264 & 50.8 & 896 & 22.6 & 5 & 13.3 & 967 & 8.5 & 61 & 21.7 & 3,334 & 16.9 \\
\hline $45-49$ & 17 & 11.6 & 80 & 6.2 & 1,180 & 45.5 & 722 & 20.2 & 2 & 5.6 & 1,085 & 8.3 & 70 & 27.1 & 3,156 & 15.1 \\
\hline $50-54$ & 12 & 7.6 & 50 & 4.3 & 1,135 & 42.3 & 590 & 19.0 & 6 & 17.2 & 1,116 & 7.7 & 50 & 20.9 & 2,959 & 13.5 \\
\hline $55-59$ & 9 & 5.8 & 34 & 3.3 & 832 & 32.2 & 300 & 11.8 & 0 & 0.0 & 709 & 4.6 & 39 & 17.4 & 1,923 & 8.7 \\
\hline $60-64$ & 7 & 5.4 & 20 & 2.2 & 506 & 23.8 & 164 & 8.5 & 0 & 0.0 & 374 & 2.6 & 18 & 10.0 & 1,089 & 5.6 \\
\hline$\geq 65$ & 4 & 1.5 & 21 & 1.0 & 363 & 8.3 & 139 & 3.5 & 3 & 6.1 & 297 & 0.8 & 14 & 3.9 & 841 & 1.7 \\
\hline \multicolumn{17}{|l|}{ Transmission category $^{c}$} \\
\hline \multicolumn{17}{|l|}{ Male adult or adolescent } \\
\hline Male-to-male sexual contact & 152 & - & 739 & - & 10,223 & - & 7,425 & - & 31 & - & 7,390 & - & 610 & - & 26,569 & - \\
\hline Injection drug use & 17 & - & 17 & - & 471 & - & 299 & - & 0 & - & 458 & - & 23 & - & 1,285 & - \\
\hline $\begin{array}{l}\text { Male-to-male sexual contact } \\
\text { and injection drug use }\end{array}$ & 21 & - & 9 & - & 254 & - & 270 & - & 5 & - & 597 & - & 46 & - & 1,201 & - \\
\hline Heterosexual contact ${ }^{d}$ & 8 & - & 56 & - & 1,926 & - & 581 & - & 3 & - & 424 & - & 40 & - & 3,037 & - \\
\hline Other ${ }^{\mathrm{e}}$ & 1 & - & 3 & - & 16 & - & 7 & - & 0 & - & 10 & - & 0 & - & 38 & - \\
\hline Subtotal & 198 & 20.9 & 824 & 11.6 & 12,890 & 82.8 & 8,582 & 38.8 & 39 & 16.8 & 8,879 & 10.6 & 719 & 33.8 & 32,131 & 24.3 \\
\hline \multicolumn{17}{|l|}{ Female adult or adolescent } \\
\hline Injection drug use & 14 & - & 7 & - & 338 & - & 139 & - & 2 & - & 411 & - & 29 & - & 939 & - \\
\hline Heterosexual contact ${ }^{d}$ & 31 & - & 136 & - & 4,189 & - & 1,025 & - & 7 & - & 1,032 & - & 121 & - & 6,541 & - \\
\hline Other ${ }^{\mathrm{e}}$ & 0 & - & 2 & - & 33 & - & 4 & - & 0 & - & 8 & - & 3 & - & 49 & - \\
\hline Subtotal & 45 & 4.5 & 145 & 1.8 & 4,560 & 26.2 & 1,168 & 5.3 & 9 & 3.9 & 1,450 & 1.7 & 152 & 6.7 & 7,529 & 5.4 \\
\hline \multicolumn{17}{|l|}{ Child (<13 yrs at diagnosis) } \\
\hline Perinatal & 0 & - & 3 & - & 64 & - & 15 & - & 0 & - & 13 & - & 4 & - & 99 & - \\
\hline Other ${ }^{f}$ & 0 & - & 5 & - & 14 & - & 1 & - & 0 & - & 3 & - & 0 & - & 23 & - \\
\hline Subtotal & 0 & 0.0 & 8 & 0.3 & 78 & 1.1 & 16 & 0.1 & 0 & 0.0 & 16 & 0.1 & 4 & 0.2 & 122 & 0.2 \\
\hline \multicolumn{17}{|l|}{ Region of residence $^{\mathrm{g}}$} \\
\hline Northeast & 9 & 7.1 & 201 & 5.4 & 2,479 & 39.0 & 1,935 & 24.1 & 5 & 23.5 & 1,475 & 4.0 & 205 & 22.1 & 6,309 & 11.2 \\
\hline Midwest & 20 & 4.9 & 103 & 4.7 & 2,511 & 35.6 & 591 & 11.2 & 4 & 12.4 & 1,718 & 3.3 & 121 & 9.4 & 5,068 & 7.5 \\
\hline South & 66 & 8.3 & 233 & 5.6 & 11,062 & 47.4 & 4,194 & 19.6 & 9 & 10.3 & 4,634 & 6.6 & 390 & 17.5 & 20,588 & 16.8 \\
\hline West & 148 & 13.9 & 440 & 5.7 & 1,476 & 42.3 & 3,046 & 13.4 & 30 & 7.0 & 2,518 & 6.5 & 159 & 6.9 & 7,817 & 10.2 \\
\hline Total & 243 & 10.2 & 977 & 5.5 & 17,528 & 43.6 & 9,766 & 17.0 & 48 & 8.5 & 10,345 & 5.2 & 875 & 12.9 & 39,782 & 12.3 \\
\hline \multicolumn{17}{|c|}{$\begin{array}{l}\text { Note. Data for the year } 2016 \text { are prelimina } \\
\text { be interpreted with caution. }\end{array}$} \\
\hline \multicolumn{17}{|c|}{$a_{\text {Hispanics/Latinos can be of any race. }}$} \\
\hline \multicolumn{17}{|c|}{ b Rates are per 100,000 population. Rates are not calculated by transmission category because of the lack of denominator data. } \\
\hline \multirow{2}{*}{\multicolumn{17}{|c|}{$\begin{array}{l}c^{c} \text { Data have been statistically adjusted to account for missing transmission category; therefor } \\
d^{d} \text { Heterosexual contact with a person known to have, or to be at high risk for, HIV infection. }\end{array}$}} \\
\hline & & & & & & & & & & & & & & & & \\
\hline e Includes hemophilia, blood trans & sfusion, & erinatal ex & osure, & nd risk fa & tor not rep & orted or & ident & & & & & & & & & \\
\hline f Includes hemophilia, blood trans & sfusion & d rick f & &  & & & & & & & & & & & & \\
\hline
\end{tabular}


Table 3b. Diagnoses of HIV infection, by race/ethnicity and selected characteristics, 2016-United States and 6 dependent areas

\begin{tabular}{|c|c|c|c|c|c|c|c|c|}
\hline & $\begin{array}{c}\text { American } \\
\text { Indian/ } \\
\text { Alaska Native } \\
\text { No. }\end{array}$ & $\begin{array}{l}\text { Asian } \\
\text { No. }\end{array}$ & $\begin{array}{c}\text { Black/African } \\
\text { American } \\
\text { No. }\end{array}$ & $\begin{array}{c}\text { Hispanic/ } \\
\text { Latino }^{a} \\
\text { No. }\end{array}$ & $\begin{array}{c}\text { Native } \\
\text { Hawaiian/ } \\
\text { Other Pacific } \\
\text { Islander } \\
\text { No. }\end{array}$ & $\begin{array}{l}\text { White } \\
\text { No. }\end{array}$ & $\begin{array}{c}\text { Multiple races } \\
\text { No. }\end{array}$ & $\begin{array}{l}\text { Total } \\
\text { No. }\end{array}$ \\
\hline \multicolumn{9}{|l|}{ Age at diagnosis (yr) } \\
\hline$<13$ & 0 & 8 & 78 & 16 & 0 & 16 & 4 & 122 \\
\hline $13-14$ & 0 & 3 & 15 & 3 & 0 & 0 & 2 & 23 \\
\hline $15-19$ & 3 & 19 & 1,029 & 347 & 2 & 222 & 43 & 1,665 \\
\hline $20-24$ & 36 & 128 & 3,585 & 1,690 & 5 & 1,215 & 189 & 6,848 \\
\hline $25-29$ & 61 & 197 & 3,661 & 2,150 & 11 & 1,773 & 177 & 8,030 \\
\hline $30-34$ & 42 & 175 & 2,202 & 1,751 & 11 & 1,465 & 120 & 5,766 \\
\hline $35-39$ & 24 & 130 & 1,680 & 1,274 & 8 & 1,108 & 88 & 4,312 \\
\hline $40-44$ & 28 & 113 & 1,265 & 952 & 5 & 967 & 61 & 3,391 \\
\hline $45-49$ & 17 & 80 & 1,181 & 778 & 2 & 1,086 & 70 & 3,214 \\
\hline $50-54$ & 12 & 50 & 1,135 & 640 & 6 & 1,116 & 50 & 3,009 \\
\hline $55-59$ & 9 & 34 & 832 & 346 & 1 & 710 & 39 & 1,971 \\
\hline $60-64$ & 7 & 20 & 507 & 188 & 0 & 374 & 18 & 1,114 \\
\hline$\geq 65$ & 4 & 21 & 363 & 157 & 3 & 297 & 14 & 859 \\
\hline \multicolumn{9}{|l|}{ Transmission category } \\
\hline \multicolumn{9}{|l|}{ Male adult or adolescent } \\
\hline Male-to-male sexual contact & 152 & 740 & 10,226 & 7,689 & 35 & 7,392 & 610 & 26,844 \\
\hline Injection drug use & 17 & 17 & 471 & 345 & 1 & 459 & 23 & 1,333 \\
\hline $\begin{array}{l}\text { Male-to-male sexual contact } \\
\text { and injection drug use }\end{array}$ & 21 & 9 & 254 & 278 & 6 & 597 & 46 & 1,210 \\
\hline Heterosexual contact ${ }^{c}$ & 8 & 56 & 1,927 & 680 & 3 & 424 & 40 & 3,138 \\
\hline Other ${ }^{d}$ & 1 & 3 & 16 & 7 & 0 & 10 & 0 & 38 \\
\hline Subtotal & 198 & 825 & 12,895 & 8,999 & 45 & 8,882 & 719 & 32,563 \\
\hline \multicolumn{9}{|l|}{ Female adult or adolescent } \\
\hline Injection drug use & 14 & 7 & 338 & 152 & 2 & 411 & 29 & 952 \\
\hline Heterosexual $\operatorname{contact}^{\mathrm{C}}$ & 31 & 136 & 4,189 & 1,121 & 7 & 1,033 & 121 & 6,638 \\
\hline Other $^{d}$ & 0 & 2 & 33 & 4 & 0 & 8 & 3 & 49 \\
\hline Subtotal & 45 & 145 & 4,560 & 1,277 & 9 & 1,451 & 152 & 7,639 \\
\hline \multicolumn{9}{|l|}{ Child (<13 yrs at diagnosis) } \\
\hline Perinatal & 0 & 3 & 64 & 15 & 0 & 13 & 4 & 99 \\
\hline Other ${ }^{\mathrm{e}}$ & 0 & 5 & 14 & 1 & 0 & 3 & 0 & 23 \\
\hline Subtotal & 0 & 8 & 78 & 16 & 0 & 16 & 4 & 122 \\
\hline \multicolumn{9}{|l|}{ Region of residence ${ }^{f}$} \\
\hline Northeast & 9 & 201 & 2,479 & 1,935 & 5 & 1,475 & 205 & 6,309 \\
\hline Midwest & 20 & 103 & 2,511 & 591 & 4 & 1,718 & 121 & 5,068 \\
\hline South & 66 & 233 & 11,062 & 4,194 & 9 & 4,634 & 390 & 20,588 \\
\hline West & 148 & 440 & 1,476 & 3,046 & 30 & 2,518 & 159 & 7,817 \\
\hline U.S. dependent areas & 0 & 1 & 5 & 526 & 6 & 4 & 0 & 542 \\
\hline Total & 243 & 978 & 17,533 & 10,292 & 54 & 10,349 & 875 & 40,324 \\
\hline
\end{tabular}







Table 4b. Stage 3 (AIDS), by race/ethnicity and selected characteristics, 2016-United States and 6 dependent areas




Table 5a. Diagnoses of HIV infection attributed to male-to-male sexual contact and male-to-male sexual contact and injection drug use, by selected characteristics, 2011-2016-United States

\begin{tabular}{|c|c|c|c|c|c|c|c|c|c|c|c|c|}
\hline & \multicolumn{6}{|c|}{ Male-to-male sexual contact } & \multicolumn{6}{|c|}{ Male-to-male sexual contact and injection drug use } \\
\hline & 2011 & 2012 & 2013 & 2014 & 2015 & 2016 & 2011 & 2012 & 2013 & 2014 & 2015 & 2016 \\
\hline & No. & No. & No. & No. & No. & No. & No. & No. & No. & No. & No. & No. \\
\hline & \multicolumn{12}{|c|}{ American Indian/Alaska Native } \\
\hline \multicolumn{13}{|c|}{ Age at diagnosis (yr) } \\
\hline $13-14$ & 0 & 0 & 0 & 0 & 0 & 0 & 0 & 0 & 0 & 0 & 0 & 0 \\
\hline $15-19$ & 6 & 4 & 1 & 4 & 6 & 3 & 0 & 1 & 0 & 0 & 1 & 0 \\
\hline $20-24$ & 13 & 21 & 17 & 32 & 31 & 27 & 4 & 1 & 1 & 1 & 4 & 4 \\
\hline $25-29$ & 14 & 15 & 23 & 28 & 24 & 42 & 1 & 5 & 1 & 1 & 1 & 8 \\
\hline $30-34$ & 7 & 15 & 13 & 18 & 17 & 31 & 1 & 3 & 2 & 0 & 0 & 4 \\
\hline $35-39$ & 14 & 10 & 7 & 6 & 14 & 12 & 0 & 3 & 1 & 0 & 4 & 2 \\
\hline $40-44$ & 6 & 14 & 8 & 12 & 6 & 15 & 1 & 1 & 0 & 1 & 0 & 0 \\
\hline $45-49$ & 9 & 11 & 7 & 10 & 8 & 10 & 2 & 1 & 1 & 2 & 0 & 0 \\
\hline $50-54$ & 2 & 6 & 7 & 8 & 5 & 6 & 0 & 1 & 1 & 2 & 0 & 1 \\
\hline $55-59$ & 2 & 1 & 0 & 2 & 4 & 3 & 1 & 0 & 0 & 0 & 0 & 1 \\
\hline $60-64$ & 2 & 0 & 1 & 0 & 0 & 4 & 0 & 0 & 0 & 0 & 0 & 0 \\
\hline$\geq 65$ & 1 & 0 & 2 & 1 & 0 & 1 & 0 & 0 & 0 & 0 & 0 & 0 \\
\hline \multicolumn{13}{|c|}{ Region of residence ${ }^{a}$} \\
\hline Northeast & 0 & 5 & 1 & 4 & 3 & 6 & 0 & 1 & 0 & 0 & 0 & 0 \\
\hline Midwest & 6 & 6 & 6 & 10 & 10 & 14 & 2 & 2 & 1 & 3 & 1 & 2 \\
\hline South & 22 & 30 & 22 & 30 & 36 & 42 & 0 & 2 & 1 & 0 & 3 & 8 \\
\hline West & 46 & 55 & 56 & 77 & 65 & 89 & 8 & 11 & 5 & 4 & 7 & 12 \\
\hline \multirow[t]{2}{*}{ Total } & 74 & 96 & 85 & 121 & 114 & 151 & 10 & 16 & 7 & 7 & 11 & 22 \\
\hline & \multicolumn{12}{|c|}{ Asian } \\
\hline \multicolumn{13}{|c|}{ Age at diagnosis (yr) } \\
\hline $13-14$ & 0 & 0 & 0 & 1 & 1 & 0 & 0 & 0 & 0 & 0 & 0 & 0 \\
\hline $15-19$ & 12 & 11 & 8 & 15 & 22 & 19 & 0 & 0 & 0 & 1 & 0 & 0 \\
\hline $20-24$ & 85 & 77 & 100 & 127 & 127 & 108 & 4 & 0 & 1 & 3 & 4 & 3 \\
\hline $25-29$ & 94 & 127 & 110 & 143 & 173 & 172 & 1 & 2 & 3 & 3 & 2 & 1 \\
\hline $30-34$ & 86 & 85 & 99 & 119 & 108 & 131 & 3 & 5 & 1 & 2 & 2 & 1 \\
\hline $35-39$ & 92 & 96 & 81 & 99 & 75 & 85 & 5 & 1 & 2 & 3 & 3 & 2 \\
\hline $40-44$ & 70 & 70 & 89 & 77 & 83 & 89 & 4 & 5 & 2 & 5 & 3 & 0 \\
\hline $45-49$ & 51 & 51 & 55 & 62 & 64 & 58 & 2 & 1 & 1 & 1 & 2 & 1 \\
\hline $50-54$ & 23 & 25 & 20 & 33 & 38 & 33 & 1 & 0 & 0 & 1 & 1 & 1 \\
\hline $55-59$ & 10 & 9 & 12 & 10 & 14 & 20 & 1 & 0 & 0 & 0 & 0 & 0 \\
\hline $60-64$ & 8 & 7 & 6 & 6 & 4 & 13 & 0 & 0 & 0 & 0 & 0 & 0 \\
\hline$\geq 65$ & 2 & 6 & 4 & 8 & 6 & 12 & 0 & 0 & 0 & 0 & 0 & 0 \\
\hline \multicolumn{13}{|c|}{ Region of residence $^{a}$} \\
\hline Northeast & 93 & 103 & 92 & 124 & 108 & 146 & 3 & 5 & 4 & 1 & 3 & 1 \\
\hline Midwest & 42 & 39 & 49 & 53 & 85 & 70 & 0 & 2 & 0 & 6 & 2 & 3 \\
\hline South & 128 & 111 & 140 & 146 & 164 & 160 & 4 & 2 & 1 & 3 & 6 & 0 \\
\hline West & 269 & 311 & 302 & 377 & 358 & 363 & 15 & 7 & 6 & 10 & 8 & 5 \\
\hline Total & 532 & 564 & 583 & 700 & 715 & 739 & 22 & 16 & 11 & 20 & 19 & 9 \\
\hline
\end{tabular}


Table 5a. Diagnoses of HIV infection attributed to male-to-male sexual contact and male-to-male sexual contact and injection drug use, by selected characteristics, 2011-2016-United States (cont)

\begin{tabular}{|c|c|c|c|c|c|c|c|c|c|c|c|c|}
\hline & \multicolumn{6}{|c|}{ Male-to-male sexual contact } & \multicolumn{6}{|c|}{ Male-to-male sexual contact and injection drug use } \\
\hline & 2011 & 2012 & 2013 & 2014 & 2015 & 2016 & 2011 & 2012 & 2013 & 2014 & 2015 & 2016 \\
\hline & No. & No. & No. & No. & No. & No. & No. & No. & No. & No. & No. & No. \\
\hline & \multicolumn{12}{|c|}{ Black/African American } \\
\hline \multicolumn{13}{|c|}{ Age at diagnosis (yr) } \\
\hline $13-14$ & 5 & 9 & 11 & 5 & 8 & 4 & 0 & 1 & 0 & 0 & 0 & 0 \\
\hline 15-19 & 896 & 880 & 811 & 803 & 866 & 801 & 17 & 14 & 14 & 15 & 12 & 16 \\
\hline 20-24 & 2,992 & 3,018 & 3,075 & 3,185 & 3,023 & 2,914 & 82 & 53 & 68 & 53 & 57 & 47 \\
\hline $25-29$ & 1,857 & 1,904 & 2,080 & 2,340 & 2,529 & 2,700 & 62 & 40 & 57 & 51 & 41 & 59 \\
\hline $30-34$ & 1,044 & 1,020 & 1,078 & 1,204 & 1,244 & 1,293 & 35 & 44 & 37 & 40 & 36 & 40 \\
\hline $35-39$ & 682 & 690 & 671 & 693 & 751 & 777 & 42 & 32 & 20 & 24 & 35 & 30 \\
\hline 40-44 & 738 & 686 & 606 & 530 & 493 & 513 & 32 & 23 & 26 & 22 & 18 & 14 \\
\hline $45-49$ & 639 & 596 & 537 & 483 & 471 & 420 & 34 & 33 & 28 & 16 & 17 & 15 \\
\hline 50-54 & 464 & 407 & 381 & 346 & 360 & 388 & 37 & 25 & 21 & 21 & 10 & 14 \\
\hline $55-59$ & 237 & 215 & 200 & 235 & 215 & 231 & 25 & 10 & 15 & 15 & 14 & 7 \\
\hline $60-64$ & 90 & 116 & 103 & 105 & 89 & 114 & 7 & 13 & 8 & 10 & 4 & 8 \\
\hline$\geq 65$ & 76 & 67 & 66 & 80 & 82 & 68 & 3 & 3 & 3 & 3 & 4 & 4 \\
\hline \multicolumn{13}{|c|}{ Region of residence ${ }^{a}$} \\
\hline Northeast & 1,327 & 1,270 & 1,236 & 1,275 & 1,236 & 1,190 & 50 & 50 & 42 & 42 & 27 & 30 \\
\hline Midwest & 1,535 & 1,620 & 1,619 & 1,620 & 1,581 & 1,627 & 62 & 51 & 46 & 42 & 37 & 52 \\
\hline South & 6,049 & 5,955 & 6,000 & 6,304 & 6,460 & 6,478 & 222 & 151 & 170 & 141 & 144 & 134 \\
\hline West & 808 & 763 & 764 & 810 & 853 & 928 & 41 & 38 & 41 & 44 & 40 & 38 \\
\hline Total & 9,719 & 9,608 & 9,619 & 10,009 & 10,130 & 10,223 & 375 & 290 & 299 & 269 & 248 & 254 \\
\hline
\end{tabular}

Age at diagnosis (yr)

\begin{tabular}{|c|c|c|c|c|c|c|c|c|c|c|c|c|}
\hline $13-14$ & 6 & 2 & 2 & 5 & 1 & 1 & 0 & 1 & 0 & 0 & 0 & 0 \\
\hline $15-19$ & 289 & 303 & 261 & 288 & 284 & 285 & 16 & 13 & 9 & 17 & 11 & 11 \\
\hline $20-24$ & 1,182 & 1,333 & 1,283 & 1,457 & 1,478 & 1,401 & 60 & 66 & 55 & 40 & 56 & 46 \\
\hline 25-29 & 1,262 & 1,345 & 1,397 & 1,509 & 1,575 & 1,771 & 76 & 64 & 69 & 56 & 69 & 55 \\
\hline $30-34$ & 1,030 & 1,171 & 1,107 & 1,220 & 1,200 & 1,324 & 72 & 71 & 54 & 60 & 56 & 58 \\
\hline 35-39 & 801 & 753 & 767 & 888 & 929 & 908 & 44 & 43 & 41 & 43 & 34 & 39 \\
\hline $40-44$ & 735 & 686 & 653 & 665 & 614 & 615 & 33 & 42 & 32 & 28 & 27 & 28 \\
\hline $45-49$ & 524 & 540 & 516 & 552 & 544 & 481 & 30 & 27 & 16 & 24 & 18 & 8 \\
\hline $50-54$ & 300 & 292 & 306 & 293 & 355 & 368 & 10 & 14 & 13 & 10 & 9 & 14 \\
\hline $55-59$ & 133 & 129 & 161 & 140 & 169 & 148 & 9 & 5 & 7 & 8 & 6 & 6 \\
\hline $60-64$ & 57 & 75 & 64 & 75 & 59 & 74 & 3 & 4 & 2 & 2 & 2 & 4 \\
\hline$\geq 65$ & 59 & 48 & 50 & 54 & 57 & 52 & 2 & 0 & 3 & 2 & 2 & 2 \\
\hline \multicolumn{13}{|c|}{ Region of residence ${ }^{a}$} \\
\hline Northeast & 1,335 & 1,372 & 1,362 & 1,420 & 1,353 & 1,256 & 76 & 81 & 71 & 51 & 46 & 39 \\
\hline Midwest & 434 & 502 & 481 & 501 & 477 & 456 & 24 & 26 & 24 & 15 & 25 & 23 \\
\hline South & 2,446 & 2,548 & 2,573 & 2,802 & 3,025 & 3,223 & 113 & 104 & 90 & 102 & 99 & 94 \\
\hline West & 2,161 & 2,255 & 2,151 & 2,423 & 2,409 & 2,491 & 142 & 139 & 114 & 122 & 119 & 113 \\
\hline Total & 6,376 & 6,677 & 6,567 & 7,146 & 7,264 & 7,426 & 355 & 350 & 299 & 290 & 289 & 269 \\
\hline
\end{tabular}


Table 5a. Diagnoses of HIV infection attributed to male-to-male sexual contact and male-to-male sexual contact and injection drug use, by selected characteristics, 2011-2016-United States (cont)

\begin{tabular}{|c|c|c|c|c|c|c|c|c|c|c|c|}
\hline \multicolumn{6}{|c|}{ Male-to-male sexual contact } & \multicolumn{6}{|c|}{ Male-to-male sexual contact and injection drug use } \\
\hline 2011 & 2012 & 2013 & 2014 & 2015 & 2016 & 2011 & 2012 & 2013 & 2014 & 2015 & 2016 \\
\hline No. & No. & No. & No. & No. & No. & No. & No. & No. & No. & No. & No. \\
\hline
\end{tabular}

Age at diagnosis (yr)

$13-14$
$15-19$
$20-24$
$25-29$
$30-34$
$35-39$
$40-44$
$45-49$
$50-54$
$55-59$
$60-64$
$\geq 65$

Native Hawaiian/Other Pacific Islander

Region of residence ${ }^{a}$

\begin{tabular}{|c|c|c|c|c|c|c|c|c|c|c|c|c|}
\hline Northeast & 2 & 3 & 2 & 0 & 2 & 4 & 0 & 0 & 0 & 0 & 0 & 1 \\
\hline Midwest & 0 & 1 & 2 & 1 & 1 & 3 & 0 & 1 & 0 & 0 & 0 & 0 \\
\hline South & 8 & 7 & 3 & 7 & 19 & 5 & 0 & 0 & 1 & 0 & 1 & 0 \\
\hline West & 32 & 31 & 28 & 24 & 38 & 19 & 2 & 3 & 1 & 1 & 1 & 4 \\
\hline Total & 42 & 42 & 35 & 32 & 60 & 31 & 2 & 4 & 2 & 1 & 2 & 5 \\
\hline
\end{tabular}

Age at diagnosis (yr)

\begin{tabular}{|c|c|c|c|c|c|c|c|c|c|c|c|c|}
\hline $13-14$ & 1 & 2 & 1 & 3 & 0 & 0 & 0 & 0 & 0 & 0 & 0 & 0 \\
\hline $15-19$ & 182 & 144 & 137 & 143 & 154 & 165 & 8 & 10 & 11 & 10 & 15 & 11 \\
\hline $20-24$ & 983 & 983 & 981 & 992 & 988 & 929 & 88 & 86 & 98 & 71 & 92 & 79 \\
\hline $25-29$ & 1,141 & 1,192 & 1,133 & 1,292 & 1,309 & 1,305 & 117 & 108 & 123 & 142 & 141 & 124 \\
\hline 30-34 & 1,035 & 1,122 & 1,043 & 1,026 & 1,044 & 1,035 & 89 & 113 & 96 & 111 & 114 & 113 \\
\hline $35-39$ & 894 & 835 & 794 & 841 & 766 & 745 & 89 & 85 & 71 & 75 & 91 & 76 \\
\hline $40-44$ & 1,141 & 1,055 & 932 & 819 & 716 & 669 & 68 & 77 & 74 & 74 & 47 & 60 \\
\hline $45-49$ & 1,152 & 1,150 & 1,044 & 954 & 776 & 786 & 66 & 78 & 48 & 59 & 47 & 52 \\
\hline $50-54$ & 809 & 838 & 775 & 830 & 814 & 811 & 47 & 48 & 36 & 46 & 58 & 38 \\
\hline $55-59$ & 473 & 472 & 509 & 462 & 428 & 489 & 15 & 25 & 23 & 20 & 30 & 26 \\
\hline $60-64$ & 250 & 232 & 298 & 237 & 257 & 249 & 13 & 8 & 13 & 10 & 10 & 11 \\
\hline$\geq 65$ & 183 & 203 & 230 & 204 & 195 & 208 & 7 & 6 & 8 & 3 & 4 & 8 \\
\hline \multicolumn{13}{|c|}{ Region of residence $^{a}$} \\
\hline Northeast & 1,295 & 1,265 & 1,164 & 1,155 & 1,036 & 1,028 & 73 & 80 & 69 & 59 & 71 & 64 \\
\hline Midwest & 1,424 & 1,413 & 1,383 & 1,322 & 1,276 & 1,224 & 99 & 104 & 77 & 99 & 112 & 107 \\
\hline South & 3,430 & 3,451 & 3,380 & 3,364 & 3,176 & 3,303 & 201 & 212 & 240 & 243 & 242 & 222 \\
\hline West & 2,095 & 2,100 & 1,952 & 1,961 & 1,959 & 1,835 & 235 & 247 & 215 & 218 & 225 & 203 \\
\hline Total & 8,244 & 8,229 & 7,879 & 7,802 & 7,447 & 7,390 & 608 & 643 & 601 & 619 & 650 & 596 \\
\hline
\end{tabular}


Table 5a. Diagnoses of HIV infection attributed to male-to-male sexual contact and male-to-male sexual contact and injection drug use, by selected characteristics, 2011-2016-United States (cont)

\begin{tabular}{|c|c|c|c|c|c|c|c|c|c|c|c|c|}
\hline & \multicolumn{6}{|c|}{ Male-to-male sexual contact } & \multicolumn{6}{|c|}{ Male-to-male sexual contact and injection drug use } \\
\hline & 2011 & 2012 & 2013 & 2014 & 2015 & 2016 & 2011 & 2012 & 2013 & 2014 & 2015 & 2016 \\
\hline & No. & No. & No. & No. & No. & No. & No. & No. & No. & No. & No. & No. \\
\hline & \multicolumn{12}{|c|}{ Multiple races } \\
\hline \multicolumn{13}{|c|}{ Age at diagnosis (yr) } \\
\hline $13-14$ & 1 & 2 & 0 & 0 & 0 & 0 & 0 & 0 & 0 & 0 & 0 & 0 \\
\hline $15-19$ & 77 & 70 & 62 & 62 & 41 & 33 & 0 & 3 & 6 & 3 & 2 & 3 \\
\hline 20-24 & 263 & 285 & 264 & 207 & 187 & 155 & 19 & 19 & 18 & 18 & 10 & 9 \\
\hline 25-29 & 191 & 197 & 198 & 177 & 187 & 145 & 18 & 10 & 11 & 14 & 12 & 10 \\
\hline $30-34$ & 128 & 133 & 121 & 108 & 92 & 90 & 12 & 17 & 12 & 5 & 18 & 7 \\
\hline $35-39$ & 96 & 97 & 91 & 80 & 68 & 48 & 5 & 10 & 7 & 6 & 2 & 7 \\
\hline $40-44$ & 108 & 90 & 62 & 65 & 43 & 39 & 10 & 7 & 3 & 10 & 4 & 2 \\
\hline $45-49$ & 95 & 70 & 70 & 58 & 40 & 36 & 10 & 9 & 5 & 6 & 2 & 4 \\
\hline $50-54$ & 49 & 49 & 35 & 42 & 38 & 26 & 6 & 3 & 5 & 2 & 0 & 3 \\
\hline $55-59$ & 27 & 29 & 27 & 23 & 16 & 22 & 4 & 6 & 1 & 2 & 1 & 2 \\
\hline $60-64$ & 20 & 8 & 15 & 5 & 11 & 11 & 1 & 4 & 0 & 0 & 1 & 1 \\
\hline$\geq 65$ & 11 & 10 & 10 & 7 & 8 & 5 & 0 & 0 & 1 & 0 & 0 & 0 \\
\hline \multicolumn{13}{|c|}{ Region of residence ${ }^{a}$} \\
\hline Northeast & 189 & 214 & 248 & 175 & 172 & 147 & 19 & 13 & 10 & 12 & 12 & 7 \\
\hline Midwest & 179 & 183 & 126 & 116 & 104 & 72 & 11 & 15 & 13 & 10 & 10 & 10 \\
\hline South & 516 & 476 & 409 & 385 & 307 & 265 & 29 & 33 & 29 & 22 & 15 & 20 \\
\hline West & 179 & 165 & 170 & 157 & 147 & 127 & 27 & 27 & 17 & 22 & 16 & 9 \\
\hline \multirow[t]{2}{*}{ Total } & 1,063 & 1,038 & 953 & 833 & 730 & 611 & 86 & 88 & 69 & 66 & 53 & 46 \\
\hline & \multicolumn{12}{|c|}{ Total } \\
\hline \multicolumn{13}{|c|}{ Age at diagnosis (yr) } \\
\hline $13-14$ & 12 & 15 & 14 & 14 & 10 & 5 & 1 & 2 & 0 & 0 & 0 & 0 \\
\hline $15-19$ & 1,463 & 1,412 & 1,282 & 1,315 & 1,375 & 1,308 & 42 & 41 & 40 & 46 & 43 & 40 \\
\hline $20-24$ & 5,528 & 5,727 & 5,723 & 6,006 & 5,846 & 5,535 & 258 & 225 & 242 & 185 & 223 & 188 \\
\hline $25-29$ & 4,567 & 4,784 & 4,950 & 5,491 & 5,808 & 6,143 & 275 & 231 & 266 & 266 & 265 & 259 \\
\hline $30-34$ & 3,335 & 3,558 & 3,470 & 3,700 & 3,717 & 3,908 & 213 & 254 & 202 & 220 & 226 & 225 \\
\hline 35-39 & 2,588 & 2,485 & 2,413 & 2,615 & 2,609 & 2,580 & 184 & 174 & 141 & 150 & 170 & 155 \\
\hline $40-44$ & 2,803 & 2,603 & 2,352 & 2,171 & 1,957 & 1,943 & 148 & 156 & 137 & 140 & 99 & 103 \\
\hline $45-49$ & 2,469 & 2,421 & 2,233 & 2,122 & 1,907 & 1,790 & 143 & 150 & 100 & 107 & 86 & 80 \\
\hline $50-54$ & 1,649 & 1,620 & 1,526 & 1,553 & 1,614 & 1,634 & 102 & 91 & 76 & 82 & 79 & 70 \\
\hline $55-59$ & 883 & 858 & 909 & 872 & 849 & 913 & 55 & 46 & 46 & 45 & 51 & 42 \\
\hline $60-64$ & 426 & 438 & 487 & 429 & 420 & 464 & 23 & 28 & 23 & 22 & 17 & 25 \\
\hline$\geq 65$ & 332 & 334 & 361 & 354 & 347 & 347 & 12 & 10 & 15 & 7 & 10 & 14 \\
\hline \multicolumn{13}{|c|}{ Region of residence $^{a}$} \\
\hline Northeast & 4,241 & 4,232 & 4,105 & 4,154 & 3,910 & 3,776 & 221 & 230 & 196 & 165 & 158 & 143 \\
\hline Midwest & 3,620 & 3,764 & 3,664 & 3,622 & 3,533 & 3,466 & 197 & 201 & 160 & 175 & 186 & 196 \\
\hline South & 12,600 & 12,578 & 12,527 & 13,038 & 13,186 & 13,477 & 568 & 503 & 533 & 511 & 509 & 478 \\
\hline West & 5,591 & 5,680 & 5,422 & 5,828 & 5,830 & 5,851 & 469 & 473 & 399 & 420 & 416 & 385 \\
\hline Total & 26,052 & 26,254 & 25,719 & 26,642 & 26,459 & 26,570 & 1,455 & 1,407 & 1,288 & 1,271 & 1,270 & 1,201 \\
\hline
\end{tabular}

Note. Data for the year 2016 are preliminary (subject to change) because they are based on only a 6-month reporting delay. Data for the year 2016 should not be used when assessing trends. Numbers less than 12, and trends based on these numbers, should be interpreted with caution. Data have been statistically adjusted to account for missing transmission category; therefore, values may not sum to column total.

${ }^{a}$ Data are based on residence at time of diagnosis of HIV infection.

$\mathrm{b}_{\text {Hispanics/Latinos can be of any race. }}$ 
Table 5b. Diagnoses of HIV infection attributed to male-to-male sexual contact and male-to-male sexual contact and injection drug use, by selected characteristics, 2011-2016-United States and 6 dependent areas

\begin{tabular}{|c|c|c|c|c|c|c|c|c|c|c|c|}
\hline \multicolumn{6}{|c|}{ Male-to-male sexual contact } & \multicolumn{6}{|c|}{ Male-to-male sexual contact and injection drug use } \\
\hline 2011 & 2012 & 2013 & 2014 & 2015 & 2016 & 2011 & 2012 & 2013 & 2014 & 2015 & 2016 \\
\hline No. & No. & No. & No. & No. & No. & No. & No. & No. & No. & No. & No. \\
\hline
\end{tabular}

American Indian/Alaska Native

Age at diagnosis (yr)

$13-14$
$15-19$
$20-24$
$25-29$
$30-34$
$35-39$
$40-44$
$45-49$
$50-54$
$55-59$
$60-64$
$\geq 65$

$\begin{array}{rrrr}0 & 0 & 0 & 0 \\ 6 & 4 & 1 & 4 \\ 13 & 21 & 17 & 32 \\ 14 & 15 & 23 & 28 \\ 7 & 15 & 13 & 18 \\ 14 & 10 & 7 & 6 \\ 6 & 14 & 8 & 12 \\ 9 & 11 & 7 & 10 \\ 2 & 6 & 7 & 8 \\ 2 & 1 & 0 & 2 \\ 2 & 0 & 1 & 0 \\ 1 & 0 & 2 & 1\end{array}$

$\begin{array}{rr}0 & 0 \\ 6 & 3 \\ 31 & 27 \\ 24 & 42 \\ 17 & 31 \\ 14 & 12 \\ 6 & 15 \\ 8 & 10 \\ 5 & 6 \\ 4 & 3 \\ 0 & 4 \\ 0 & 1\end{array}$

Region of residence ${ }^{a}$

Northeast

Midwest

South

0

$5 \quad 1$

4
10
30
77
0

West

$\begin{array}{rr}6 & 6 \\ 22 & 30\end{array}$

6

$\begin{array}{rr}3 & 6 \\ 10 & 14 \\ 36 & 42 \\ 65 & 89 \\ 0 & 0\end{array}$

Total

$74 \quad 96$

85

\section{Age at diagnosis (yr)}

$13-14$
$15-19$
$20-24$
$25-29$
$30-34$
$35-39$
$40-44$
$45-49$
$50-54$
$55-59$
$60-64$
$\geq 65$

$\begin{array}{rr}0 & 0 \\ 12 & 11 \\ 85 & 77 \\ 94 & 127 \\ 86 & 85 \\ 92 & 96 \\ 70 & 70 \\ 51 & 51 \\ 23 & 25 \\ 10 & 9 \\ 8 & 7 \\ 2 & 6\end{array}$

$\begin{array}{rr}0 & 1 \\ 8 & 15 \\ 100 & 127 \\ 110 & 143 \\ 99 & 119 \\ 81 & 99 \\ 89 & 77 \\ 55 & 62 \\ 20 & 34 \\ 12 & 10 \\ 6 & 6 \\ 4 & 8\end{array}$

$\begin{array}{rr}1 & 0 \\ 22 & 19 \\ 127 & 108 \\ 175 & 172 \\ 109 & 131 \\ 76 & 86 \\ 83 & 89 \\ 64 & 58 \\ 38 & 33 \\ 14 & 20 \\ 4 & 13 \\ 6 & 12\end{array}$

Asian

Region of residence ${ }^{a}$

Northeast

Midwest

South

West

U.S. dependent areas

Total

$\begin{array}{rrrrrrr}93 & 103 & 92 & 124 & 108 & 146 & \\ 42 & 39 & 49 & 53 & 85 & 70 & \\ 128 & 111 & 140 & 146 & 164 & 160 & \\ 269 & 311 & 302 & 377 & 358 & 363 & 15 \\ 0 & 0 & 0 & 1 & 4 & 1 & \\ 532 & \mathbf{5 6 4} & \mathbf{5 8 3} & \mathbf{7 0 1} & \mathbf{7 1 9} & \mathbf{7 4 0} & \mathbf{2}\end{array}$

0
0
4
1
1
0
1
2
0
1
0
0

$\begin{array}{ll}0 & 0 \\ 1 & 0 \\ 1 & 1 \\ 5 & 1 \\ 3 & 2 \\ 3 & 1 \\ 1 & 0 \\ 1 & 1 \\ 1 & 1 \\ 0 & 0 \\ 0 & 0 \\ 0 & 0\end{array}$

$\begin{array}{lll}0 & 0 & 0 \\ 0 & 1 & 0 \\ 1 & 4 & 4 \\ 1 & 1 & 8 \\ 0 & 0 & 4 \\ 0 & 4 & 2 \\ 1 & 0 & 0 \\ 2 & 0 & 0 \\ 2 & 0 & 1 \\ 0 & 0 & 1 \\ 0 & 0 & 0 \\ 0 & 0 & 0\end{array}$


Table 5b. Diagnoses of HIV infection attributed to male-to-male sexual contact and male-to-male sexual contact and injection drug use, by selected characteristics, 2011-2016-United States and 6 dependent areas (cont)

\begin{tabular}{|c|c|c|c|c|c|c|c|c|c|c|c|c|}
\hline & \multicolumn{6}{|c|}{ Male-to-male sexual contact } & \multicolumn{6}{|c|}{ Male-to-male sexual contact and injection drug use } \\
\hline & 2011 & 2012 & 2013 & 2014 & 2015 & 2016 & 2011 & 2012 & 2013 & 2014 & 2015 & 2016 \\
\hline & No. & No. & No. & No. & No. & No. & No. & No. & No. & No. & No. & No. \\
\hline & \multicolumn{12}{|c|}{ Black/African American } \\
\hline \multicolumn{13}{|l|}{ Age at diagnosis (yr) } \\
\hline $13-14$ & 5 & 9 & 11 & 5 & 8 & 4 & 0 & 1 & 0 & 0 & 0 & 0 \\
\hline 15-19 & 896 & 880 & 811 & 803 & 866 & 801 & 17 & 14 & 14 & 15 & 12 & 16 \\
\hline 20-24 & 2,993 & 3,018 & 3,076 & 3,185 & 3,024 & 2,915 & 82 & 53 & 68 & 53 & 57 & 47 \\
\hline $25-29$ & 1,858 & 1,904 & 2,081 & 2,340 & 2,530 & 2,700 & 62 & 40 & 57 & 51 & 41 & 59 \\
\hline $30-34$ & 1,044 & 1,020 & 1,078 & 1,205 & 1,244 & 1,294 & 35 & 44 & 37 & 40 & 36 & 40 \\
\hline $35-39$ & 683 & 690 & 672 & 694 & 751 & 777 & 42 & 32 & 20 & 24 & 35 & 30 \\
\hline 40-44 & 738 & 687 & 607 & 530 & 494 & 514 & 32 & 23 & 26 & 22 & 18 & 14 \\
\hline $45-49$ & 639 & 596 & 537 & 484 & 472 & 420 & 34 & 33 & 28 & 16 & 17 & 15 \\
\hline $50-54$ & 464 & 407 & 381 & 346 & 360 & 388 & 37 & 25 & 21 & 21 & 10 & 14 \\
\hline $55-59$ & 238 & 215 & 200 & 236 & 215 & 231 & 25 & 10 & 15 & 15 & 14 & 7 \\
\hline $60-64$ & 90 & 116 & 103 & 105 & 89 & 114 & 8 & 13 & 8 & 10 & 4 & 8 \\
\hline$\geq 65$ & 76 & 67 & 66 & 80 & 82 & 68 & 3 & 3 & 4 & 3 & 4 & 4 \\
\hline \multicolumn{13}{|l|}{ Region of residence ${ }^{a}$} \\
\hline Northeast & 1,327 & 1,270 & 1,236 & 1,275 & 1,236 & 1,190 & 50 & 50 & 42 & 42 & 27 & 30 \\
\hline Midwest & 1,535 & 1,620 & 1,619 & 1,620 & 1,581 & 1,627 & 62 & 51 & 46 & 42 & 37 & 52 \\
\hline South & 6,049 & 5,955 & 6,000 & 6,304 & 6,460 & 6,478 & 222 & 151 & 170 & 141 & 144 & 134 \\
\hline West & 808 & 763 & 764 & 810 & 853 & 928 & 41 & 38 & 41 & 44 & 40 & 38 \\
\hline U.S. dependent areas & 4 & 1 & 4 & 4 & 5 & 3 & 1 & 0 & 0 & 0 & 0 & 0 \\
\hline Total & 9,723 & 9,609 & 9,623 & 10,013 & 10,135 & 10,226 & 376 & 290 & 299 & 269 & 248 & 254 \\
\hline
\end{tabular}

Age at diagnosis (yr)

\begin{tabular}{|c|c|c|c|c|c|c|c|c|c|c|c|c|}
\hline $13-14$ & 7 & 2 & 2 & 5 & 1 & 1 & 0 & 1 & 0 & 0 & 0 & 0 \\
\hline $15-19$ & 297 & 314 & 276 & 305 & 287 & 293 & 18 & 13 & 9 & 17 & 11 & 11 \\
\hline 20-24 & 1,234 & 1,406 & 1,348 & 1,541 & 1,535 & 1,457 & 64 & 69 & 56 & 43 & 59 & 46 \\
\hline $25-29$ & 1,313 & 1,409 & 1,452 & 1,573 & 1,640 & 1,817 & 77 & 70 & 70 & 57 & 71 & 57 \\
\hline $30-34$ & 1,065 & 1,216 & 1,157 & 1,251 & 1,233 & 1,364 & 72 & 76 & 59 & 61 & 57 & 60 \\
\hline $35-39$ & 835 & 773 & 788 & 923 & 962 & 943 & 49 & 48 & 41 & 45 & 38 & 40 \\
\hline $40-44$ & 761 & 714 & 682 & 697 & 642 & 635 & 34 & 44 & 36 & 29 & 30 & 29 \\
\hline $45-49$ & 554 & 567 & 532 & 570 & 570 & 494 & 34 & 28 & 18 & 24 & 19 & 10 \\
\hline $50-54$ & 319 & 307 & 321 & 305 & 367 & 391 & 11 & 19 & 17 & 11 & 10 & 14 \\
\hline $55-59$ & 145 & 135 & 164 & 147 & 178 & 163 & 9 & 6 & 7 & 8 & 6 & 6 \\
\hline $60-64$ & 62 & 80 & 74 & 80 & 66 & 78 & 4 & 4 & 2 & 2 & 2 & 4 \\
\hline$\geq 65$ & 63 & 53 & 54 & 58 & 65 & 52 & 2 & 0 & 3 & 3 & 4 & 2 \\
\hline \multicolumn{13}{|l|}{ Region of residence ${ }^{a}$} \\
\hline Northeast & 1,335 & 1,372 & 1,362 & 1,420 & 1,353 & 1,256 & 76 & 81 & 71 & 51 & 46 & 39 \\
\hline Midwest & 434 & 502 & 481 & 501 & 477 & 456 & 24 & 26 & 24 & 15 & 25 & 23 \\
\hline South & 2,446 & 2,548 & 2,573 & 2,802 & 3,025 & 3,223 & 113 & 104 & 90 & 102 & 99 & 94 \\
\hline West & 2,161 & 2,255 & 2,151 & 2,423 & 2,409 & 2,491 & 142 & 139 & 114 & 122 & 119 & 113 \\
\hline U.S. dependent areas & 275 & 298 & 284 & 309 & 281 & 264 & 19 & 26 & 17 & 9 & 18 & 8 \\
\hline Total & 6,651 & 6,975 & 6,851 & 7,455 & 7,545 & 7,690 & 374 & 376 & 316 & 299 & 307 & 277 \\
\hline
\end{tabular}


Table 5b. Diagnoses of HIV infection attributed to male-to-male sexual contact and male-to-male sexual contact and injection drug use, by selected characteristics, 2011-2016-United States and 6 dependent areas (cont)

\begin{tabular}{|c|c|c|c|c|c|c|c|c|c|c|c|c|}
\hline & \multicolumn{6}{|c|}{ Male-to-male sexual contact } & \multicolumn{6}{|c|}{ Male-to-male sexual contact and injection drug use } \\
\hline & 2011 & 2012 & 2013 & 2014 & 2015 & 2016 & 2011 & 2012 & 2013 & 2014 & 2015 & 2016 \\
\hline & No. & No. & No. & No. & No. & No. & No. & No. & No. & No. & No. & No. \\
\hline & \multicolumn{12}{|c|}{ Native Hawaiian/Other Pacific Islander } \\
\hline \multicolumn{13}{|l|}{ Age at diagnosis (yr) } \\
\hline $13-14$ & 0 & 0 & 0 & 0 & 0 & 0 & 0 & 0 & 0 & 0 & 0 & 0 \\
\hline $15-19$ & 1 & 1 & 1 & 1 & 2 & 2 & 1 & 0 & 0 & 0 & 1 & 0 \\
\hline $20-24$ & 10 & 12 & 3 & 6 & 12 & 4 & 1 & 0 & 0 & 0 & 0 & 0 \\
\hline $25-29$ & 9 & 4 & 9 & 3 & 13 & 8 & 0 & 1 & 2 & 0 & 0 & 3 \\
\hline $30-34$ & 6 & 12 & 11 & 7 & 12 & 7 & 0 & 2 & 0 & 1 & 0 & 2 \\
\hline $35-39$ & 9 & 4 & 3 & 7 & 6 & 5 & 0 & 0 & 0 & 0 & 1 & 0 \\
\hline $40-44$ & 5 & 1 & 3 & 4 & 3 & 5 & 0 & 0 & 0 & 0 & 0 & 0 \\
\hline $45-49$ & 0 & 3 & 4 & 3 & 6 & 1 & 0 & 1 & 0 & 0 & 0 & 0 \\
\hline $50-54$ & 1 & 3 & 2 & 1 & 5 & 2 & 0 & 0 & 0 & 0 & 0 & 0 \\
\hline $55-59$ & 1 & 3 & 0 & 0 & 3 & 1 & 0 & 0 & 0 & 0 & 0 & 0 \\
\hline $60-64$ & 0 & 1 & 0 & 1 & 0 & 0 & 0 & 0 & 0 & 0 & 0 & 0 \\
\hline$\geq 65$ & 0 & 0 & 0 & 1 & 0 & 2 & 0 & 0 & 0 & 0 & 0 & 1 \\
\hline \multicolumn{13}{|l|}{ Region of residence ${ }^{a}$} \\
\hline Northeast & 2 & 3 & 2 & 0 & 2 & 4 & 0 & 0 & 0 & 0 & 0 & 1 \\
\hline Midwest & 0 & 1 & 2 & 1 & 1 & 3 & 0 & 1 & 0 & 0 & 0 & 0 \\
\hline South & 8 & 7 & 3 & 7 & 19 & 5 & 0 & 0 & 1 & 0 & 1 & 0 \\
\hline West & 32 & 31 & 28 & 24 & 38 & 19 & 2 & 3 & 1 & 1 & 1 & 4 \\
\hline U.S. dependent areas & 0 & 1 & 1 & 2 & 3 & 5 & 0 & 0 & 0 & 0 & 0 & 1 \\
\hline \multirow[t]{2}{*}{ Total } & 42 & 43 & 36 & 34 & 63 & 36 & 2 & 4 & 2 & 1 & 2 & 6 \\
\hline & \multicolumn{12}{|c|}{ White } \\
\hline \multicolumn{13}{|l|}{ Age at diagnosis (yr) } \\
\hline $13-14$ & 1 & 2 & 1 & 3 & 0 & 0 & 0 & 0 & 0 & 0 & 0 & 0 \\
\hline $15-19$ & 182 & 144 & 137 & 143 & 154 & 165 & 8 & 10 & 11 & 10 & 15 & 11 \\
\hline $20-24$ & 983 & 983 & 981 & 993 & 988 & 930 & 88 & 86 & 98 & 71 & 92 & 79 \\
\hline $25-29$ & 1,141 & 1,192 & 1,133 & 1,293 & 1,309 & 1,305 & 117 & 108 & 123 & 142 & 141 & 124 \\
\hline $30-34$ & 1,035 & 1,122 & 1,044 & 1,027 & 1,044 & 1,035 & 89 & 113 & 96 & 111 & 114 & 113 \\
\hline $35-39$ & 894 & 835 & 794 & 841 & 766 & 745 & 89 & 85 & 71 & 75 & 91 & 76 \\
\hline $40-44$ & 1,141 & 1,056 & 932 & 819 & 716 & 669 & 68 & 77 & 74 & 74 & 47 & 60 \\
\hline $45-49$ & 1,152 & 1,150 & 1,044 & 954 & 776 & 787 & 66 & 78 & 48 & 59 & 47 & 52 \\
\hline $50-54$ & 809 & 838 & 775 & 830 & 815 & 811 & 47 & 48 & 36 & 46 & 58 & 38 \\
\hline $55-59$ & 473 & 472 & 509 & 463 & 428 & 489 & 15 & 25 & 23 & 20 & 30 & 26 \\
\hline $60-64$ & 250 & 232 & 298 & 237 & 257 & 249 & 13 & 8 & 13 & 10 & 10 & 11 \\
\hline$\geq 65$ & 183 & 203 & 230 & 204 & 195 & 208 & 7 & 6 & 8 & 3 & 4 & 8 \\
\hline \multicolumn{13}{|l|}{ Region of residence ${ }^{a}$} \\
\hline Northeast & 1,295 & 1,265 & 1,164 & 1,155 & 1,036 & 1,028 & 73 & 80 & 69 & 59 & 71 & 64 \\
\hline Midwest & 1,424 & 1,413 & 1,383 & 1,322 & 1,276 & 1,224 & 99 & 104 & 77 & 99 & 112 & 107 \\
\hline South & 3,430 & 3,451 & 3,380 & 3,364 & 3,176 & 3,303 & 201 & 212 & 240 & 243 & 242 & 222 \\
\hline West & 2,095 & 2,100 & 1,952 & 1,961 & 1,959 & 1,835 & 235 & 247 & 215 & 218 & 225 & 203 \\
\hline U.S. dependent areas & 0 & 1 & 1 & 4 & 1 & 2 & 0 & 0 & 0 & 0 & 0 & 0 \\
\hline Total & 8,244 & 8,230 & 7,880 & 7,806 & 7,448 & 7,392 & 608 & 643 & 601 & 619 & 650 & 596 \\
\hline
\end{tabular}


Table 5b. Diagnoses of HIV infection attributed to male-to-male sexual contact and male-to-male sexual contact and injection drug use, by selected characteristics, 2011-2016-United States and 6 dependent areas (cont)

\begin{tabular}{|c|c|c|c|c|c|c|c|c|c|c|c|c|}
\hline & \multicolumn{6}{|c|}{ Male-to-male sexual contact } & \multicolumn{6}{|c|}{ Male-to-male sexual contact and injection drug use } \\
\hline & 2011 & 2012 & 2013 & 2014 & 2015 & 2016 & 2011 & 2012 & 2013 & 2014 & 2015 & 2016 \\
\hline & No. & No. & No. & No. & No. & No. & No. & No. & No. & No. & No. & No. \\
\hline & \multicolumn{12}{|c|}{ Multiple races } \\
\hline \multicolumn{13}{|l|}{ Age at diagnosis (yr) } \\
\hline $13-14$ & 1 & 2 & 0 & 0 & 0 & 0 & 0 & 0 & 0 & 0 & 0 & 0 \\
\hline $15-19$ & 77 & 70 & 62 & 63 & 41 & 33 & 0 & 3 & 6 & 3 & 2 & 3 \\
\hline 20-24 & 263 & 285 & 264 & 207 & 187 & 155 & 19 & 19 & 18 & 18 & 10 & 9 \\
\hline $25-29$ & 191 & 197 & 198 & 177 & 187 & 145 & 18 & 10 & 11 & 14 & 12 & 10 \\
\hline $30-34$ & 128 & 134 & 121 & 108 & 92 & 90 & 12 & 17 & 12 & 5 & 18 & 7 \\
\hline $35-39$ & 96 & 97 & 91 & 80 & 68 & 48 & 5 & 10 & 7 & 6 & 2 & 7 \\
\hline 40-44 & 108 & 90 & 62 & 65 & 43 & 39 & 10 & 7 & 3 & 10 & 4 & 2 \\
\hline $45-49$ & 95 & 70 & 70 & 58 & 40 & 36 & 10 & 9 & 5 & 6 & 2 & 4 \\
\hline $50-54$ & 49 & 49 & 35 & 42 & 38 & 26 & 6 & 3 & 5 & 2 & 0 & 3 \\
\hline $55-59$ & 27 & 29 & 27 & 23 & 16 & 22 & 4 & 6 & 1 & 2 & 1 & 2 \\
\hline $60-64$ & 20 & 8 & 15 & 5 & 11 & 11 & 1 & 4 & 0 & 0 & 1 & 1 \\
\hline$\geq 65$ & 11 & 10 & 10 & 7 & 8 & 5 & 0 & 0 & 1 & 0 & 0 & 0 \\
\hline \multicolumn{13}{|l|}{ Region of residence ${ }^{a}$} \\
\hline Northeast & 189 & 214 & 248 & 175 & 172 & 147 & 19 & 13 & 10 & 12 & 12 & 7 \\
\hline Midwest & 179 & 183 & 126 & 116 & 104 & 72 & 11 & 15 & 13 & 10 & 10 & 10 \\
\hline South & 516 & 476 & 409 & 385 & 307 & 265 & 29 & 33 & 29 & 22 & 15 & 20 \\
\hline West & 179 & 165 & 170 & 157 & 147 & 127 & 27 & 27 & 17 & 22 & 16 & 9 \\
\hline U.S. dependent areas & 0 & 1 & 0 & 1 & 0 & 0 & 0 & 0 & 0 & 0 & 0 & 0 \\
\hline \multirow[t]{2}{*}{ Total } & 1,063 & 1,039 & 953 & 834 & 730 & 611 & 86 & 88 & 69 & 66 & 53 & 46 \\
\hline & \multicolumn{12}{|c|}{ Total } \\
\hline \multicolumn{13}{|l|}{ Age at diagnosis (yr) } \\
\hline $13-14$ & 13 & 15 & 14 & 14 & 10 & 5 & 1 & 2 & 0 & 0 & 0 & 0 \\
\hline $15-19$ & 1,471 & 1,423 & 1,297 & 1,333 & 1,378 & 1,316 & 44 & 41 & 40 & 46 & 43 & 40 \\
\hline $20-24$ & 5,581 & 5,800 & 5,788 & 6,091 & 5,905 & 5,595 & 261 & 228 & 243 & 187 & 226 & 188 \\
\hline $25-29$ & 4,618 & 4,848 & 5,007 & 5,557 & 5,877 & 6,190 & 277 & 236 & 267 & 267 & 267 & 262 \\
\hline $30-34$ & 3,370 & 3,604 & 3,522 & 3,734 & 3,750 & 3,951 & 213 & 259 & 207 & 220 & 228 & 227 \\
\hline $35-39$ & 2,622 & 2,505 & 2,435 & 2,650 & 2,643 & 2,616 & 189 & 179 & 141 & 152 & 175 & 156 \\
\hline $40-44$ & 2,828 & 2,633 & 2,383 & 2,205 & 1,987 & 1,965 & 149 & 157 & 141 & 141 & 102 & 104 \\
\hline $45-49$ & 2,499 & 2,448 & 2,250 & 2,141 & 1,934 & 1,805 & 147 & 151 & 102 & 107 & 87 & 82 \\
\hline $50-54$ & 1,667 & 1,635 & 1,541 & 1,565 & 1,628 & 1,657 & 103 & 96 & 80 & 83 & 80 & 71 \\
\hline $55-59$ & 896 & 864 & 912 & 881 & 857 & 929 & 55 & 47 & 46 & 45 & 51 & 42 \\
\hline $60-64$ & 431 & 443 & 496 & 433 & 428 & 468 & 25 & 28 & 23 & 22 & 17 & 25 \\
\hline$\geq 65$ & 336 & 339 & 365 & 359 & 355 & 347 & 12 & 10 & 15 & 8 & 12 & 14 \\
\hline \multicolumn{13}{|l|}{ Region of residence ${ }^{a}$} \\
\hline Northeast & 4,241 & 4,232 & 4,105 & 4,154 & 3,910 & 3,776 & 221 & 230 & 196 & 165 & 158 & 143 \\
\hline Midwest & 3,620 & 3,764 & 3,664 & 3,622 & 3,533 & 3,466 & 197 & 201 & 160 & 175 & 186 & 196 \\
\hline South & 12,600 & 12,578 & 12,527 & 13,038 & 13,186 & 13,477 & 568 & 503 & 533 & 511 & 509 & 478 \\
\hline West & 5,591 & 5,680 & 5,422 & 5,828 & 5,830 & 5,851 & 469 & 473 & 399 & 420 & 416 & 385 \\
\hline U.S. dependent areas & 280 & 302 & 290 & 320 & 293 & 274 & 20 & 26 & 18 & 9 & 18 & 9 \\
\hline Total & 26,332 & 26,556 & 26,009 & 26,962 & 26,753 & 26,844 & 1,476 & 1,434 & 1,306 & 1,280 & 1,288 & 1,210 \\
\hline
\end{tabular}

Note. Data for the year 2016 are preliminary (subject to change) because they are based on only a 6-month reporting delay. Data for the year 2016 should not be used when assessing trends. Numbers less than 12 , and trends based on these numbers, should be interpreted with caution. Data have been statistically adjusted to account for missing transmission category; therefore, values may not sum to column total.



$\mathrm{b}_{\text {Hispanics/Latinos can be of any race. }}$ 
Table 6a. Diagnoses of HIV infection attributed to injection drug use, by selected characteristics, 2011-2016-United States

\begin{tabular}{|c|c|c|c|c|c|c|c|c|c|c|c|}
\hline \multicolumn{6}{|c|}{ Male } & \multicolumn{6}{|c|}{ Female } \\
\hline 2011 & 2012 & 2013 & 2014 & 2015 & 2016 & 2011 & 2012 & 2013 & 2014 & 2015 & 2016 \\
\hline No. & No. & No. & No. & No. & No. & No. & No. & No. & No. & No. & No. \\
\hline
\end{tabular}

American Indian/Alaska Native

Age at diagnosis (yr)

$13-14$
$15-19$
$20-24$
$25-29$
$30-34$
$35-39$
$40-44$
$45-49$
$50-54$
$55-59$
$60-64$
$\geq 65$

$\begin{array}{llll}0 & 0 & 0 & 0 \\ 0 & 0 & 0 & 0 \\ 0 & 2 & 2 & 1 \\ 2 & 2 & 1 & 2 \\ 0 & 1 & 2 & 0 \\ 3 & 0 & 1 & 0 \\ 0 & 5 & 3 & 3 \\ 2 & 0 & 3 & 2 \\ 1 & 0 & 1 & 3 \\ 2 & 2 & 1 & 0 \\ 0 & 0 & 0 & 0 \\ 0 & 0 & 1 & 0\end{array}$

$\begin{array}{ll}0 & 0 \\ 0 & 0 \\ 0 & 3 \\ 2 & 4 \\ 2 & 0 \\ 1 & 1 \\ 0 & 2 \\ 0 & 2 \\ 1 & 0 \\ 1 & 3 \\ 0 & 1 \\ 0 & 0\end{array}$

Region of residence ${ }^{a}$

\begin{tabular}{|c|c|c|c|c|c|c|c|c|c|c|c|c|}
\hline Northeast & 0 & 1 & 0 & 1 & 0 & 0 & 0 & 0 & 1 & 0 & 0 & 0 \\
\hline Midwest & 0 & 2 & 1 & 1 & 0 & 1 & 5 & 3 & 3 & 2 & 0 & 1 \\
\hline South & 2 & 1 & 4 & 4 & 2 & 4 & 4 & 1 & 2 & 4 & 4 & 1 \\
\hline West & 8 & 10 & 10 & 5 & 5 & 13 & 7 & 8 & 6 & 5 & 9 & 11 \\
\hline Total & 10 & 14 & 15 & 11 & 7 & 18 & 16 & 12 & 12 & 11 & 13 & 13 \\
\hline
\end{tabular}

Asian

Age at diagnosis (yr)

$13-14$
$15-19$
$20-24$
$25-29$
$30-34$
$35-39$
$40-44$
$45-49$
$50-54$
$55-59$
$60-64$
$\geq 65$

$\begin{array}{ll}0 & 0 \\ 0 & 1 \\ 0 & 0 \\ 0 & 1 \\ 3 & 2 \\ 4 & 6 \\ 3 & 5 \\ 3 & 1 \\ 1 & 1 \\ 1 & 1 \\ 1 & 1 \\ 0 & 1\end{array}$

$\begin{array}{ll}0 & 0 \\ 0 & 1 \\ 2 & 0 \\ 2 & 0 \\ 3 & 3 \\ 3 & 5 \\ 5 & 3 \\ 2 & 1 \\ 1 & 2 \\ 2 & 0 \\ 1 & 1 \\ 0 & 1\end{array}$

$\begin{array}{ll}0 & 0 \\ 0 & 0 \\ 0 & 0 \\ 0 & 2 \\ 6 & 2 \\ 1 & 4 \\ 3 & 1 \\ 1 & 3 \\ 2 & 2 \\ 1 & 1 \\ 1 & 1 \\ 1 & 1\end{array}$

0
0
1
2
5
4
0
0
1
1
0
0

$\begin{array}{lllll}0 & 0 & 0 & 0 & 0 \\ 0 & 0 & 0 & 0 & 0 \\ 1 & 1 & 0 & 2 & 1 \\ 0 & 0 & 1 & 2 & 2 \\ 4 & 2 & 2 & 2 & 2 \\ 2 & 0 & 2 & 1 & 2 \\ 2 & 3 & 2 & 2 & 2 \\ 3 & 1 & 1 & 2 & 1 \\ 0 & 2 & 0 & 0 & 4 \\ 1 & 0 & 0 & 1 & 0 \\ 0 & 1 & 1 & 1 & 0 \\ 0 & 0 & 0 & 0 & 0\end{array}$

Region of residence ${ }^{a}$ Northeast Midwest

South

West

Total

$\begin{array}{rr}7 & 5 \\ 2 & 0 \\ 2 & 4 \\ 4 & 9 \\ 15 & 18\end{array}$

\begin{tabular}{|c|c|}
\hline 7 & 6 \\
\hline 2 & 1 \\
\hline 6 & 7 \\
\hline 7 & 4 \\
\hline 22 & 18 \\
\hline
\end{tabular}

7
0
3
8
18

6
1
3
7
17

$\begin{array}{ll}0 & 0 \\ 1 & 0 \\ 1 & 1 \\ 1 & 3 \\ 0 & 0 \\ 2 \\ 1 \\ 0 \\ 1 \\ 0 \\ 0 \\ 0\end{array}$

$\begin{array}{lllll}0 & 0 & 0 & 0 & 0 \\ 0 & 0 & 0 & 0 & 0 \\ 1 & 0 & 0 & 1 & 2 \\ 3 & 0 & 1 & 0 & 1 \\ 0 & 1 & 0 & 1 & 1 \\ 2 & 0 & 2 & 0 & 2 \\ 0 & 1 & 1 & 1 & 0 \\ 0 & 2 & 1 & 0 & 1 \\ 0 & 1 & 1 & 0 & 1 \\ 0 & 3 & 1 & 0 & 0 \\ 0 & 0 & 1 & 1 & 0 \\ 0 & 0 & 0 & 0 & 0\end{array}$


Table 6a. Diagnoses of HIV infection attributed to injection drug use, by selected characteristics, 2011-2016-United States (cont)

\begin{tabular}{|c|c|c|c|c|c|c|c|c|c|c|c|c|}
\hline & \multicolumn{6}{|c|}{ Male } & \multicolumn{6}{|c|}{ Female } \\
\hline & 2011 & 2012 & 2013 & 2014 & 2015 & 2016 & 2011 & 2012 & 2013 & 2014 & 2015 & 2016 \\
\hline & No. & No. & No. & No. & No. & No. & No. & No. & No. & No. & No. & No. \\
\hline & \multicolumn{12}{|c|}{ Black/African American } \\
\hline \multicolumn{13}{|c|}{ Age at diagnosis (yr) } \\
\hline $13-14$ & 0 & 0 & 0 & 0 & 0 & 0 & 1 & 1 & 0 & 0 & 2 & 0 \\
\hline $15-19$ & 9 & 8 & 7 & 6 & 5 & 6 & 15 & 14 & 10 & 9 & 6 & 10 \\
\hline 20-24 & 28 & 35 & 30 & 37 & 35 & 29 & 41 & 46 & 31 & 30 & 25 & 25 \\
\hline $25-29$ & 39 & 43 & 43 & 42 & 43 & 46 & 51 & 42 & 32 & 40 & 44 & 36 \\
\hline $30-34$ & 54 & 56 & 48 & 41 & 44 & 38 & 52 & 57 & 45 & 36 & 33 & 39 \\
\hline $35-39$ & 64 & 53 & 50 & 39 & 41 & 55 & 59 & 56 & 37 & 42 & 33 & 36 \\
\hline $40-44$ & 100 & 70 & 52 & 48 & 47 & 48 & 78 & 66 & 48 & 45 & 35 & 32 \\
\hline $45-49$ & 131 & 99 & 102 & 70 & 49 & 53 & 108 & 73 & 61 & 50 & 44 & 30 \\
\hline $50-54$ & 126 & 122 & 92 & 65 & 70 & 68 & 83 & 89 & 59 & 48 & 45 & 52 \\
\hline $55-59$ & 115 & 91 & 88 & 79 & 62 & 55 & 62 & 54 & 59 & 45 & 33 & 39 \\
\hline $60-64$ & 54 & 52 & 47 & 44 & 37 & 42 & 23 & 29 & 25 & 19 & 24 & 25 \\
\hline$\geq 65$ & 40 & 40 & 31 & 41 & 32 & 32 & 16 & 20 & 13 & 17 & 15 & 14 \\
\hline \multicolumn{13}{|c|}{ Region of residence ${ }^{a}$} \\
\hline Northeast & 231 & 203 & 186 & 162 & 129 & 132 & 151 & 145 & 93 & 103 & 86 & 77 \\
\hline Midwest & 79 & 70 & 63 & 48 & 50 & 49 & 82 & 67 & 59 & 45 & 50 & 49 \\
\hline South & 401 & 349 & 293 & 273 & 239 & 238 & 312 & 293 & 237 & 197 & 169 & 174 \\
\hline West & 49 & 47 & 48 & 30 & 47 & 51 & 45 & 41 & 31 & 37 & 34 & 37 \\
\hline Total & 760 & 669 & 590 & 513 & 465 & 470 & 590 & 546 & 420 & 382 & 339 & 337 \\
\hline & \multicolumn{12}{|c|}{ Hispanic/Latino ${ }^{b}$} \\
\hline \multicolumn{13}{|c|}{ Age at diagnosis (yr) } \\
\hline $13-14$ & 0 & 0 & 0 & 0 & 0 & 0 & 0 & 0 & 0 & 0 & 0 & 0 \\
\hline $15-19$ & 2 & 3 & 3 & 2 & 1 & 2 & 7 & 5 & 5 & 5 & 4 & 2 \\
\hline $20-24$ & 25 & 12 & 20 & 16 & 26 & 18 & 13 & 18 & 14 & 17 & 22 & 11 \\
\hline $25-29$ & 47 & 35 & 37 & 32 & 35 & 34 & 24 & 19 & 23 & 15 & 14 & 28 \\
\hline 30-34 & 63 & 57 & 51 & 45 & 49 & 47 & 32 & 28 & 21 & 23 & 17 & 18 \\
\hline $35-39$ & 55 & 58 & 42 & 36 & 46 & 37 & 25 & 24 & 15 & 19 & 16 & 15 \\
\hline 40-44 & 68 & 55 & 44 & 50 & 41 & 31 & 25 & 19 & 22 & 27 & 15 & 16 \\
\hline $45-49$ & 75 & 52 & 60 & 50 & 37 & 31 & 38 & 24 & 19 & 16 & 17 & 13 \\
\hline $50-54$ & 46 & 51 & 35 & 36 & 39 & 41 & 17 & 17 & 18 & 17 & 16 & 16 \\
\hline $55-59$ & 36 & 27 & 37 & 28 & 30 & 26 & 9 & 12 & 10 & 14 & 8 & 11 \\
\hline $60-64$ & 12 & 23 & 11 & 12 & 11 & 13 & 6 & 6 & 6 & 7 & 4 & 5 \\
\hline$\geq 65$ & 14 & 18 & 13 & 9 & 14 & 18 & 5 & 3 & 6 & 6 & 6 & 5 \\
\hline \multicolumn{13}{|c|}{ Region of residence ${ }^{a}$} \\
\hline Northeast & 187 & 170 & 145 & 108 & 120 & 100 & 83 & 87 & 72 & 69 & 53 & 48 \\
\hline Midwest & 34 & 21 & 20 & 11 & 19 & 11 & 11 & 9 & 11 & 11 & 6 & 8 \\
\hline South & 123 & 119 & 95 & 95 & 101 & 94 & 66 & 43 & 41 & 44 & 46 & 45 \\
\hline West & 98 & 84 & 91 & 102 & 90 & 93 & 43 & 36 & 33 & 42 & 34 & 38 \\
\hline Total & 442 & 394 & 351 & 316 & 330 & 298 & 203 & 175 & 157 & 166 & 139 & 139 \\
\hline
\end{tabular}


Table 6a. Diagnoses of HIV infection attributed to injection drug use, by selected characteristics, 2011-2016-United States (cont)

\begin{tabular}{|c|c|c|c|c|c|c|c|c|c|c|c|}
\hline \multicolumn{6}{|c|}{ Male } & \multicolumn{6}{|c|}{ Female } \\
\hline 2011 & 2012 & 2013 & 2014 & 2015 & 2016 & 2011 & 2012 & 2013 & 2014 & 2015 & 2016 \\
\hline No. & No. & No. & No. & No. & No. & No. & No. & No. & No. & No. & No. \\
\hline
\end{tabular}

Age at diagnosis (yr)

\begin{tabular}{|c|c|c|c|c|c|c|c|c|c|c|c|}
\hline $13-14$ & 0 & 0 & 0 & 0 & 0 & 0 & 0 & 0 & 0 & 0 & 0 \\
\hline $15-19$ & 0 & 0 & 0 & 0 & 0 & 0 & 0 & 0 & 0 & 0 & 0 \\
\hline $20-24$ & 0 & 0 & 0 & 0 & 0 & 0 & 0 & 0 & 0 & 0 & 0 \\
\hline $25-29$ & 0 & 0 & 0 & 0 & 0 & 0 & 0 & 0 & 0 & 0 & 1 \\
\hline $30-34$ & 0 & 0 & 0 & 0 & 0 & 0 & 0 & 0 & 0 & 0 & 0 \\
\hline $35-39$ & 1 & 0 & 0 & 0 & 1 & 0 & 0 & 0 & 0 & 0 & 0 \\
\hline 40-44 & 0 & 0 & 0 & 0 & 1 & 0 & 0 & 0 & 0 & 0 & 0 \\
\hline $45-49$ & 1 & 0 & 1 & 0 & 0 & 0 & 0 & 0 & 0 & 0 & 0 \\
\hline $50-54$ & 0 & 0 & 0 & 0 & 0 & 0 & 0 & 0 & 0 & 0 & 0 \\
\hline $55-59$ & 0 & 0 & 0 & 0 & 1 & 0 & 0 & 0 & 0 & 0 & 1 \\
\hline $60-64$ & 1 & 0 & 0 & 1 & 0 & 0 & 0 & 0 & 0 & 0 & 0 \\
\hline$\geq 65$ & 0 & 0 & 0 & 0 & 0 & 0 & 0 & 0 & 0 & 0 & 0 \\
\hline
\end{tabular}

Region of residence ${ }^{a}$

Northeast

Midwest

South

West

0

\section{0}

Native Hawaiian/Other Pacific Islander

\section{Total}

0

3

\section{Age at diagnosis (yr)}

13-14

15-19

20-24

25-29

$30-34$

35-39

$40-44$

$45-49$

$50-54$

$55-59$

60-64

$\geq 65$

Region of residence ${ }^{a}$

Northeast

Midwest

South

West

Total

0
2
21
32
45
42
59
62
58
35
21
10

$\begin{array}{rr}0 & 0 \\ 7 & 2 \\ 31 & 28 \\ 38 & 36 \\ 47 & 48 \\ 26 & 37 \\ 46 & 47 \\ 56 & 60 \\ 57 & 50 \\ 24 & 38 \\ 13 & 17 \\ 7 & 13\end{array}$

$\begin{array}{rrr}0 & 0 & 0 \\ 3 & 2 & 4 \\ 29 & 47 & 32 \\ 37 & 82 & 77 \\ 55 & 75 & 65 \\ 47 & 51 & 69 \\ 62 & 52 & 49 \\ 52 & 67 & 50 \\ 55 & 52 & 49 \\ 31 & 34 & 33 \\ 21 & 18 & 16 \\ 11 & 10 & 13\end{array}$

\section{White}

$\begin{array}{rrrrrrrrrrrr}77 & 66 & 64 & 67 & 73 & 84 & 85 & 73 & 73 & 56 & 69 & 75 \\ 41 & 45 & 52 & 52 & 139 & 86 & 50 & 45 & 61 & 38 & 131 & 79 \\ 135 & 137 & 152 & 153 & 156 & 161 & 169 & 163 & 161 & 162 & 194 & 179 \\ 131 & 105 & 107 & 129 & 124 & 127 & 90 & 69 & 72 & 76 & 82 & 78 \\ 384 & 353 & \mathbf{3 7 5} & \mathbf{4 0 1} & \mathbf{4 9 2} & \mathbf{4 5 8} & \mathbf{3 9 4} & \mathbf{3 5 0} & \mathbf{3 6 7} & \mathbf{3 3 2} & \mathbf{4 7 6} & \mathbf{4 1 1}\end{array}$


Table 6a. Diagnoses of HIV infection attributed to injection drug use, by selected characteristics, 2011-2016-United States (cont)

\begin{tabular}{|c|c|c|c|c|c|c|c|c|c|c|c|}
\hline \multicolumn{6}{|c|}{ Male } & \multicolumn{6}{|c|}{ Female } \\
\hline 2011 & 2012 & 2013 & 2014 & 2015 & 2016 & 2011 & 2012 & 2013 & 2014 & 2015 & 2016 \\
\hline No. & No. & No. & No. & No. & No. & No. & No. & No. & No. & No. & No. \\
\hline
\end{tabular}

Age at diagnosis (yr)

\begin{tabular}{|c|c|c|c|c|c|c|c|c|c|c|c|}
\hline $13-14$ & 0 & 0 & 0 & 0 & 0 & 0 & 0 & 0 & 0 & 0 & 0 \\
\hline $15-19$ & 0 & 0 & 0 & 0 & 1 & 0 & 1 & 3 & 1 & 2 & 1 \\
\hline $20-24$ & 2 & 4 & 0 & 1 & 1 & 2 & 5 & 3 & 3 & 2 & 1 \\
\hline $25-29$ & 7 & 2 & 4 & 8 & 6 & 2 & 5 & 7 & 5 & 2 & 2 \\
\hline $30-34$ & 3 & 3 & 6 & 7 & 4 & 1 & 12 & 6 & 4 & 6 & 8 \\
\hline $35-39$ & 6 & 4 & 2 & 4 & 0 & 4 & 5 & 7 & 4 & 5 & 3 \\
\hline $40-44$ & 5 & 4 & 8 & 2 & 2 & 4 & 5 & 9 & 6 & 5 & 2 \\
\hline $45-49$ & 6 & 5 & 5 & 3 & 2 & 2 & 8 & 10 & 4 & 5 & 3 \\
\hline $50-54$ & 6 & 8 & 8 & 4 & 5 & 5 & 5 & 5 & 6 & 6 & 4 \\
\hline $55-59$ & 11 & 3 & 3 & 4 & 3 & 2 & 5 & 5 & 3 & 2 & 5 \\
\hline $60-64$ & 3 & 5 & 4 & 2 & 1 & 1 & 1 & 2 & 3 & 1 & 2 \\
\hline$\geq 65$ & 3 & 2 & 2 & 2 & 3 & 1 & 1 & 1 & 1 & 1 & 1 \\
\hline
\end{tabular}

Region of residence ${ }^{\mathrm{a}}$

Northeast

Midwest

South

$17 \quad 15$

\section{$9 \quad 9$}

7
6
11

West

$\begin{array}{rr}5 & 5 \\ 22 & 10\end{array}$

Total

$50 \quad 40 \quad 41$

29

$\begin{array}{rr}6 & 18 \\ 3 & 5 \\ 10 & 25 \\ 5 & 4\end{array}$

$\begin{array}{rr}18 & 21 \\ 5 & \\ 25 & 26 \\ 4 & \end{array}$

$\begin{array}{rrrrr}21 & 12 & 11 & 7 & 6 \\ 6 & 7 & 1 & 5 & 4 \\ 26 & 17 & 18 & 13 & 13 \\ 4 & 5 & 7 & 6 & 6 \\ 57 & 41 & 37 & 31 & \mathbf{2 9}\end{array}$

\section{Age at diagnosis (yr)}

13-14

$15-19$

20-24

25-29

30-34

$35-39$

$40-44$

$45-49$

$50-54$

$55-59$

$60-64$

$\geq 65$

Region of residence ${ }^{\mathrm{a}}$

Northeast

Midwest

South

West

Total

$\begin{array}{rrr}0 & 0 & \\ 13 & 20 & \\ 75 & 84 & \\ 126 & 122 & 123 \\ 168 & 166 & 158 \\ 175 & 148 & 1 \\ 235 & 184 & 160 \\ 278 & 213 & 233 \\ 239 & 241 & 186 \\ 200 & 149 & 168 \\ 91 & 94 & \\ 67 & 68 & \end{array}$

0
12
82
123
158
135
160
233
186
168
80
61

$\begin{array}{rr}0 & 0 \\ 11 & 9 \\ 84 & 109\end{array}$

$\begin{array}{rr}0 & 1 \\ 13 & 30\end{array}$

1

$84 \quad 95$

$122 \quad 168$

$152 \quad 181$

$164 \quad 144$

$154 \quad 153$

$\begin{array}{llll}130 & 141 & 170 & 141\end{array}$

$168 \quad 147$

$178 \quad 157$

$165 \quad 170$

$136 \quad 160$

$140 \quad 210$

$165 \quad 160$

$142 \quad 132$

$119 \quad 102$

$\begin{array}{ll}73 & 38\end{array}$

$64 \quad 60$

$65 \quad 28$

32
97

97
120

145

130

141

155

149

100

49

$\begin{array}{rrrr}1 & 0 & 3 & 1 \\ 26 & 28 & 19 & 16 \\ 86 & 86 & 100 & 75 \\ 111 & 112 & 149 & 153 \\ 130 & 127 & 144 & 133 \\ 93 & 114 & 108 & 114 \\ 128 & 113 & 119 & 93 \\ 134 & 109 & 112 & 93 \\ 126 & 97 & 106 & 116 \\ 96 & 81 & 72 & 75 \\ 47 & 35 & 43 & 44 \\ 25 & 30 & 28 & 27\end{array}$

$\begin{array}{rrr}520 & 460 & 412 \\ 162 & 143 & 140 \\ 685 & 621 & 571 \\ 300 & 265 & 274 \\ 1,666 & 1,489 & 1,39\end{array}$

$\begin{array}{rrr}412 & 352 & 337 \\ 140 & 121 & 213 \\ 571 & 542 & 513 \\ 274 & 281 & 280 \\ 1,396 & 1,297 & 1,343\end{array}$

$\begin{array}{rr}328 & 340 \\ 152 & 153 \\ 509 & 577 \\ 296 & 192 \\ 1,285 & 1,262\end{array}$

$\begin{array}{rrrrrr}340 & 328 & 251 & 241 & 216 & 208 \\ 153 & 130 & 141 & 99 & 192 & 141 \\ 577 & 528 & 461 & 425 & 427 & 415 \\ 192 & 160 & 150 & 168 & 169 & 175 \\ \mathbf{1 , 2 6 2} & \mathbf{1 , 1 4 7} & \mathbf{1 , 0 0 2} & \mathbf{9 3 3} & \mathbf{1 , 0 0 4} & \mathbf{9 3 9}\end{array}$

Note. Data for the year 2016 are preliminary (subject to change) because they are based on only a 6-month reporting delay. Data for the year 2016 should not be used when assessing trends. Numbers less than 12 , and trends based on these numbers, should be interpreted with caution. Data have been statistically adjusted to account for missing transmission category; therefore, values may not sum to column total.

${ }^{a}$ Data are based on residence at time of diagnosis of HIV infection.

$\mathrm{b}$ Hispanics/Latinos can be of any race. 
Table 6b. Diagnoses of HIV infection attributed to injection drug use, by selected characteristics, 2011-2016-United States and 6 dependent areas



\section{American Indian/Alaska Native}

\section{Age at diagnosis (yr)}

13-14

15-19

20-24

25-29

$30-34$

35-39

40-44

$45-49$

50-54

$55-59$

60-64

$\geq 65$

Region of residence ${ }^{a}$

Northeast

Midwest

South

West

U.S. dependent areas

$\begin{array}{ll}0 & 0 \\ 0 & 0 \\ 0 & 2 \\ 2 & 2 \\ 0 & 1 \\ 3 & 0 \\ 0 & 5 \\ 2 & 0 \\ 1 & 0 \\ 2 & 2 \\ 0 & 0 \\ 0 & 0\end{array}$

$\begin{array}{lll}0 & 0 & 0 \\ 0 & 0 & 0 \\ 2 & 1 & 0 \\ 1 & 2 & 2 \\ 2 & 0 & 2 \\ 1 & 0 & 1 \\ 3 & 3 & 0 \\ 3 & 2 & 0 \\ 1 & 3 & 1 \\ 1 & 0 & 1 \\ 0 & 0 & 0 \\ 1 & 0 & 0\end{array}$

$\begin{array}{ll}0 & 0 \\ 0 & 0 \\ 0 & 3 \\ 2 & 4 \\ 2 & 0 \\ 1 & 1 \\ 0 & 2 \\ 0 & 2 \\ 1 & 0 \\ 1 & 3 \\ 0 & 1 \\ 0 & 0\end{array}$

$\begin{array}{ll}0 & 0 \\ 0 & 0 \\ 3 & 1 \\ 4 & 2 \\ 0 & 5 \\ 1 & 4 \\ 2 & 0 \\ 2 & 0 \\ 0 & 1 \\ 3 & 1 \\ 1 & 0 \\ 0 & 0\end{array}$

0
0
1
0
4
2
2
3
0
1
0
0

$\begin{array}{llll}0 & 0 & 0 & 0 \\ 0 & 0 & 0 & 0 \\ 1 & 0 & 2 & 1 \\ 0 & 1 & 2 & 2 \\ 2 & 2 & 2 & 2 \\ 0 & 2 & 1 & 2 \\ 3 & 2 & 2 & 2 \\ 1 & 1 & 2 & 1 \\ 2 & 0 & 0 & 4 \\ 0 & 0 & 1 & 0 \\ 1 & 1 & 1 & 0 \\ 0 & 0 & 0 & 0\end{array}$

Total

1
2
1
10
0

$\begin{array}{rl}0 & 1 \\ 1 & 1 \\ 4 & 4 \\ 10 & 5 \\ 0 & 0\end{array}$

0
0
2
5
0

0
1
4
13
0

\begin{tabular}{rrrrrr}
0 & 0 & 1 & 0 & 0 & 0 \\
5 & 3 & 3 & 2 & 0 & 1 \\
4 & 1 & 2 & 4 & 4 & 1 \\
7 & 8 & 6 & 5 & 9 & 11 \\
0 & 0 & 0 & 0 & 0 & 0 \\
16 & 12 & 12 & 11 & 13 & 13 \\
\hline
\end{tabular}

Asian

Age at diagnosis (yr)

$13-14$
$15-19$
$20-24$
$25-29$
$30-34$
$35-39$
$40-44$
$45-49$
$50-54$
$55-59$
$60-64$
$\geq 65$

Region of residence ${ }^{a}$ Northeast

Midwest

South

West

U.S. dependent areas

Total

$\begin{array}{ll}0 & 0 \\ 0 & 1 \\ 0 & 0 \\ 0 & 1 \\ 3 & 2 \\ 4 & 6 \\ 3 & 5 \\ 3 & 1 \\ 1 & 1 \\ 1 & 1 \\ 1 & 1 \\ 0 & 1\end{array}$

$\begin{array}{ll}0 & 0 \\ 0 & 1 \\ 2 & 0 \\ 2 & 0 \\ 3 & 3 \\ 3 & 5 \\ 5 & 3 \\ 2 & 1 \\ 1 & 2 \\ 2 & 0 \\ 1 & 1 \\ 0 & 1\end{array}$

0
0
0
0
6
2
3
1
2
1
1
1

$\begin{array}{ll}0 & 0 \\ 0 & 1 \\ 0 & 1 \\ 2 & 1 \\ 2 & 0 \\ 4 & 2 \\ 1 & 1 \\ 3 & 0 \\ 2 & 1 \\ 1 & 0 \\ 1 & 0 \\ 1 & 0\end{array}$

$\begin{array}{ll}0 & 0 \\ 0 & 0 \\ 1 & 0 \\ 3 & 0 \\ 0 & 1 \\ 2 & 0 \\ 0 & 1 \\ 0 & 2 \\ 0 & 1 \\ 0 & 3 \\ 0 & 0 \\ 0 & 0\end{array}$

$\begin{array}{lll}0 & 0 & 0 \\ 0 & 0 & 0 \\ 0 & 1 & 2 \\ 1 & 0 & 1 \\ 0 & 1 & 1 \\ 2 & 0 & 2 \\ 1 & 1 & 0 \\ 1 & 0 & 1 \\ 1 & 0 & 1 \\ 1 & 0 & 0 \\ 1 & 1 & 0 \\ 0 & 0 & 0\end{array}$

$\begin{array}{rr}7 & 5 \\ 2 & 0 \\ 2 & 4 \\ 4 & 9 \\ 0 & 0 \\ 15 & 18\end{array}$

$\begin{array}{rr}7 & 6 \\ 2 & 1 \\ 6 & 7 \\ 7 & 4 \\ 0 & 0 \\ 22 & 18\end{array}$

$\begin{array}{rr}7 & 6 \\ 0 & 1 \\ 3 & 3 \\ 8 & 7 \\ 1 & 0 \\ 19 & 17\end{array}$

2
1
1
3
0
7

2
1
1
3
0
7

$\begin{array}{llll}1 & 2 & 1 & 2 \\ 1 & 2 & 1 & 0 \\ 2 & 0 & 2 & 2 \\ 4 & 3 & 2 & 2 \\ 0 & 0 & 0 & 0 \\ 8 & 7 & 6 & 6\end{array}$


Table 6b. Diagnoses of HIV infection attributed to injection drug use, by selected characteristics, 2011-2016-United States and 6 dependent areas (cont)

\begin{tabular}{|c|c|c|c|c|c|c|c|c|c|c|c|}
\hline \multicolumn{6}{|c|}{ Male } & \multicolumn{6}{|c|}{ Female } \\
\hline 2011 & 2012 & 2013 & 2014 & 2015 & 2016 & 2011 & 2012 & 2013 & 2014 & 2015 & 2016 \\
\hline No. & No. & No. & No. & No. & No. & No. & No. & No. & No. & No. & No. \\
\hline
\end{tabular}

Age at diagnosis (yr)

\begin{tabular}{|c|c|c|c|c|c|c|c|c|c|c|c|c|}
\hline $13-14$ & 0 & 0 & 0 & 0 & 0 & 0 & 1 & 1 & 0 & 0 & 2 & 0 \\
\hline $15-19$ & 9 & 8 & 7 & 6 & 5 & 6 & 15 & 14 & 10 & 9 & 6 & 10 \\
\hline $20-24$ & 28 & 35 & 30 & 37 & 35 & 29 & 41 & 46 & 31 & 30 & 25 & 25 \\
\hline $25-29$ & 39 & 43 & 43 & 42 & 43 & 46 & 51 & 42 & 32 & 40 & 44 & 36 \\
\hline 30-34 & 54 & 56 & 48 & 41 & 44 & 38 & 52 & 57 & 45 & 36 & 33 & 39 \\
\hline $35-39$ & 64 & 53 & 51 & 39 & 41 & 55 & 60 & 56 & 37 & 43 & 33 & 36 \\
\hline $40-44$ & 100 & 70 & 52 & 49 & 47 & 48 & 78 & 66 & 48 & 45 & 35 & 32 \\
\hline $45-49$ & 131 & 99 & 102 & 70 & 49 & 53 & 108 & 73 & 61 & 50 & 44 & 30 \\
\hline $50-54$ & 127 & 122 & 92 & 66 & 70 & 68 & 83 & 89 & 59 & 48 & 45 & 52 \\
\hline $55-59$ & 115 & 91 & 88 & 79 & 62 & 55 & 62 & 54 & 59 & 45 & 33 & 39 \\
\hline $60-64$ & 54 & 52 & 47 & 46 & 37 & 42 & 23 & 29 & 25 & 19 & 24 & 25 \\
\hline$\geq 65$ & 40 & 40 & 31 & 41 & 32 & 32 & 16 & 20 & 13 & 17 & 15 & 14 \\
\hline \multicolumn{13}{|l|}{ Region of residence ${ }^{a}$} \\
\hline Northeast & 231 & 203 & 186 & 162 & 129 & 132 & 151 & 145 & 93 & 103 & 86 & 77 \\
\hline Midwest & 79 & 70 & 63 & 48 & 50 & 49 & 82 & 67 & 59 & 45 & 50 & 49 \\
\hline South & 401 & 349 & 293 & 273 & 239 & 238 & 312 & 293 & 237 & 197 & 169 & 174 \\
\hline West & 49 & 47 & 48 & 30 & 47 & 51 & 45 & 41 & 31 & 37 & 34 & 37 \\
\hline U.S. dependent areas & 1 & 1 & 1 & 2 & 0 & 0 & 0 & 0 & 1 & 0 & 0 & 0 \\
\hline Total & 761 & 670 & 591 & 515 & 465 & 470 & 590 & 546 & 421 & 382 & 339 & 337 \\
\hline
\end{tabular}

Age at diagnosis (yr)

\begin{tabular}{|c|c|c|c|c|c|c|c|c|c|c|c|c|}
\hline $13-14$ & 0 & 0 & 0 & 0 & 0 & 0 & 0 & 0 & 0 & 0 & 0 & 0 \\
\hline $15-19$ & 4 & 4 & 3 & 2 & 2 & 4 & 7 & 5 & 5 & 6 & 4 & 3 \\
\hline $20-24$ & 31 & 18 & 23 & 20 & 29 & 21 & 15 & 22 & 14 & 18 & 22 & 12 \\
\hline $25-29$ & 54 & 43 & 41 & 41 & 38 & 36 & 28 & 20 & 25 & 15 & 14 & 29 \\
\hline $30-34$ & 79 & 69 & 58 & 50 & 58 & 49 & 37 & 31 & 23 & 25 & 18 & 20 \\
\hline 35-39 & 73 & 66 & 53 & 43 & 51 & 45 & 32 & 27 & 21 & 22 & 18 & 17 \\
\hline $40-44$ & 86 & 72 & 51 & 55 & 48 & 39 & 33 & 20 & 24 & 30 & 16 & 17 \\
\hline $45-49$ & 92 & 69 & 74 & 62 & 44 & 36 & 41 & 28 & 24 & 17 & 17 & 15 \\
\hline $50-54$ & 64 & 59 & 46 & 41 & 48 & 44 & 19 & 19 & 21 & 18 & 17 & 17 \\
\hline $55-59$ & 43 & 34 & 49 & 39 & 39 & 32 & 11 & 14 & 11 & 15 & 8 & 11 \\
\hline $60-64$ & 15 & 25 & 15 & 17 & 15 & 17 & 7 & 8 & 8 & 7 & 4 & 6 \\
\hline$\geq 65$ & 14 & 24 & 17 & 12 & 16 & 23 & 5 & 3 & 8 & 7 & 6 & 5 \\
\hline \multicolumn{13}{|l|}{ Region of residence ${ }^{a}$} \\
\hline Northeast & 187 & 170 & 145 & 108 & 120 & 100 & 83 & 87 & 72 & 69 & 53 & 48 \\
\hline Midwest & 34 & 21 & 20 & 11 & 19 & 11 & 11 & 9 & 11 & 11 & 6 & 8 \\
\hline South & 123 & 119 & 95 & 95 & 101 & 94 & 66 & 43 & 41 & 44 & 46 & 45 \\
\hline West & 98 & 84 & 91 & 102 & 90 & 93 & 43 & 36 & 33 & 42 & 34 & 38 \\
\hline U.S. dependent areas & 110 & 89 & 80 & 65 & 58 & 46 & 33 & 23 & 25 & 14 & 6 & 13 \\
\hline Total & 552 & 483 & 431 & 381 & 388 & 344 & 236 & 198 & 182 & 180 & 145 & 152 \\
\hline
\end{tabular}


Table 6b. Diagnoses of HIV infection attributed to injection drug use, by selected characteristics, 2011-2016-United States and 6 dependent areas (cont)

\begin{tabular}{|c|c|c|c|c|c|c|c|c|c|c|c|}
\hline \multicolumn{6}{|c|}{ Male } & \multicolumn{6}{|c|}{ Female } \\
\hline 2011 & 2012 & 2013 & 2014 & 2015 & 2016 & 2011 & 2012 & 2013 & 2014 & 2015 & 2016 \\
\hline No. & No. & No. & No. & No. & No. & No. & No. & No. & No. & No. & No. \\
\hline
\end{tabular}

Age at diagnosis (yr)

13-14

$15-19$

20-24

$25-29$

30-34

$35-39$

40-44

$45-49$

$50-54$

$55-59$

60-64

$\geq 65$

\section{Region of residence ${ }^{a}$}

Northeast

Midwest

South

West

U.S. dependent areas

Total

\section{Age at diagnosis (yr)}

13-14

15-19

20-24

25-29

30-34

35-39

40-44

45-49

50-54

55-59

60-64

$\geq 65$

Region of residence ${ }^{a}$ Northeast

Midwest

South

West

U.S. dependent areas

Total

0
0
0
0
0
1
0
1
0
0
1
0

Native Hawaiian/Other Pacific Islander

$\begin{array}{lllllllllll}0 & 0 & 0 & 0 & 0 & 0 & 0 & 0 & 0 & 0 & 0 \\ 0 & 0 & 0 & 0 & 0 & 0 & 0 & 0 & 0 & 0 & 0 \\ 0 & 0 & 0 & 0 & 0 & 0 & 0 & 0 & 0 & 0 & 0 \\ 0 & 0 & 0 & 0 & 0 & 0 & 0 & 0 & 0 & 1 & 0 \\ 0 & 0 & 0 & 0 & 0 & 0 & 0 & 0 & 0 & 0 & 0 \\ 0 & 0 & 0 & 1 & 0 & 0 & 0 & 0 & 0 & 0 & 0 \\ 0 & 0 & 0 & 1 & 0 & 0 & 0 & 0 & 0 & 0 & 0 \\ 0 & 1 & 0 & 0 & 0 & 0 & 0 & 0 & 0 & 0 & 0 \\ 0 & 0 & 0 & 0 & 0 & 0 & 0 & 0 & 0 & 0 & 2 \\ 0 & 0 & 0 & 1 & 0 & 0 & 0 & 0 & 0 & 1 & 0 \\ 0 & 0 & 1 & 0 & 0 & 0 & 0 & 0 & 0 & 0 & 0 \\ 0 & 0 & 0 & 0 & 0 & 0 & 0 & 0 & 0 & 0 & 0\end{array}$

\begin{tabular}{lllllllllllll}
0 & 0 & 0 & 1 & 1 & 0 & 0 & 0 & 0 & 0 & 0 & 0 \\
0 & 0 & 0 & 0 & 0 & 0 & 0 & 0 & 0 & 0 & 0 & 0 \\
0 & 0 & 0 & 0 & 1 & 0 & 0 & 0 & 0 & 0 & 0 & 0 \\
3 & 0 & 1 & 0 & 1 & 0 & 0 & 0 & 0 & 0 & 2 & 2 \\
0 & 0 & 0 & 0 & 0 & 0 & 0 & 0 & 0 & 0 & 0 & 0 \\
3 & 0 & 1 & 1 & 3 & 0 & 0 & 0 & 0 & 0 & 2 & $\mathbf{2}$ \\
\hline
\end{tabular}

White

$\begin{array}{rrrrrrrrrrrr}0 & 0 & 0 & 0 & 0 & 0 & 0 & 0 & 0 & 0 & 1 & 0 \\ 2 & 7 & 2 & 3 & 2 & 4 & 6 & 9 & 9 & 11 & 9 & 4 \\ 21 & 31 & 28 & 29 & 47 & 32 & 34 & 28 & 37 & 37 & 48 & 35 \\ 32 & 38 & 36 & 37 & 82 & 77 & 60 & 50 & 50 & 52 & 86 & 83 \\ 45 & 47 & 48 & 55 & 75 & 65 & 52 & 51 & 57 & 60 & 84 & 71 \\ 42 & 26 & 37 & 47 & 51 & 69 & 47 & 40 & 36 & 45 & 54 & 54 \\ 59 & 46 & 47 & 62 & 52 & 49 & 51 & 46 & 49 & 34 & 65 & 41 \\ 62 & 56 & 60 & 52 & 67 & 50 & 56 & 45 & 48 & 36 & 45 & 42 \\ 58 & 57 & 50 & 55 & 53 & 49 & 54 & 37 & 41 & 27 & 42 & 38 \\ 35 & 24 & 38 & 31 & 34 & 33 & 25 & 29 & 22 & 19 & 24 & 22 \\ 21 & 13 & 17 & 21 & 18 & 16 & 6 & 11 & 12 & 6 & 12 & 13 \\ 10 & 7 & 13 & 11 & 10 & 13 & 6 & 4 & 5 & 5 & 6\end{array}$

$\begin{array}{rrrrrrrrrrrr}77 & 66 & 64 & 67 & 73 & 84 & 85 & 73 & 73 & 56 & 69 & 75 \\ 41 & 45 & 52 & 52 & 139 & 86 & 50 & 45 & 61 & 38 & 131 & 79 \\ 135 & 137 & 152 & 153 & 156 & 161 & 169 & 163 & 161 & 162 & 194 & 179 \\ 131 & 105 & 107 & 129 & 124 & 127 & 90 & 69 & 72 & 76 & 82 & 78 \\ 1 & 0 & 0 & 0 & 0 & 1 & 0 & 0 & 0 & 0 & 0 & 0 \\ 385 & 353 & 375 & 401 & 492 & 459 & 394 & 350 & 367 & 332 & 476 & 411\end{array}$


Table 6b. Diagnoses of HIV infection attributed to injection drug use, by selected characteristics, 2011-2016-United States and 6 dependent areas (cont)

\begin{tabular}{|c|c|c|c|c|c|c|c|c|c|c|c|}
\hline \multicolumn{6}{|c|}{ Male } & \multicolumn{6}{|c|}{ Female } \\
\hline 2011 & 2012 & 2013 & 2014 & 2015 & 2016 & 2011 & 2012 & 2013 & 2014 & 2015 & 2016 \\
\hline No. & No. & No. & No. & No. & No. & No. & No. & No. & No. & No. & No. \\
\hline
\end{tabular}

Age at diagnosis (yr)

13-14

$15-19$

20-24

$25-29$

30-34

$35-39$

$40-44$

$45-49$

$50-54$

$55-59$

$60-64$

$\geq 65$

\section{Region of residence ${ }^{a}$}

Northeast

Midwest

South

West

U.S. dependent areas

Total

$\begin{array}{rl}0 & 0 \\ 0 & 0 \\ 2 & 4 \\ 7 & 2 \\ 3 & 3 \\ 6 & 4 \\ 5 & 4 \\ 6 & 5 \\ 6 & 8 \\ 11 & 3 \\ 3 & 5 \\ 3 & 2\end{array}$

Multiple races

$\begin{array}{rrrrrrrrr}0 & 0 & 0 & 0 & 0 & 0 & 0 & 0 & 0 \\ 0 & 1 & 0 & 1 & 3 & 1 & 2 & 1 & 0 \\ 1 & 1 & 2 & 5 & 3 & 3 & 2 & 1 & 2 \\ 8 & 6 & 2 & 5 & 7 & 5 & 2 & 2 & 2 \\ 7 & 4 & 1 & 12 & 6 & 4 & 6 & 8 & 2 \\ 4 & 0 & 4 & 5 & 7 & 4 & 5 & 3 & 6 \\ 2 & 2 & 4 & 5 & 9 & 6 & 5 & 2 & 3 \\ 3 & 2 & 2 & 8 & 10 & 4 & 5 & 3 & 6 \\ 4 & 5 & 5 & 5 & 5 & 6 & 6 & 4 & 4 \\ 4 & 3 & 2 & 5 & 5 & 3 & 2 & 5 & 3 \\ 2 & 1 & 1 & 1 & 2 & 3 & 1 & 2 & 1 \\ 2 & 3 & 1 & 1 & 1 & 1 & 1 & 1 & 0\end{array}$

Age at diagnosis (yr)

\begin{tabular}{|c|c|c|c|c|c|c|c|c|c|c|c|c|}
\hline $13-14$ & 0 & 0 & 0 & 0 & 0 & 0 & 1 & 1 & 1 & 0 & 3 & 1 \\
\hline $15-19$ & 15 & 21 & 12 & 12 & 10 & 15 & 30 & 32 & 26 & 29 & 19 & 17 \\
\hline $20-24$ & 81 & 90 & 85 & 88 & 111 & 88 & 97 & 101 & 86 & 87 & 100 & 76 \\
\hline $25-29$ & 133 & 130 & 127 & 130 & 171 & 165 & 148 & 121 & 113 & 112 & 149 & 154 \\
\hline $30-34$ & 184 & 178 & 165 & 156 & 189 & 156 & 158 & 148 & 132 & 130 & 145 & 135 \\
\hline $35-39$ & 192 & 155 & 146 & 137 & 148 & 178 & 148 & 133 & 99 & 118 & 110 & 116 \\
\hline $40-44$ & 253 & 201 & 167 & 173 & 154 & 144 & 168 & 143 & 130 & 116 & 120 & 94 \\
\hline $45-49$ & 297 & 230 & 247 & 190 & 164 & 146 & 213 & 159 & 139 & 110 & 112 & 95 \\
\hline $50-54$ & 257 & 248 & 198 & 171 & 179 & 168 & 162 & 151 & 129 & 98 & 107 & 117 \\
\hline $55-59$ & 207 & 156 & 181 & 152 & 141 & 126 & 104 & 102 & 97 & 82 & 72 & 76 \\
\hline $60-64$ & 93 & 96 & 84 & 87 & 72 & 77 & 38 & 51 & 49 & 35 & 44 & 45 \\
\hline$\geq 65$ & 67 & 74 & 65 & 67 & 62 & 70 & 28 & 28 & 27 & 31 & 28 & 27 \\
\hline \multicolumn{13}{|l|}{ Region of residence ${ }^{a}$} \\
\hline Northeast & 520 & 460 & 412 & 352 & 337 & 328 & 340 & 328 & 251 & 241 & 216 & 208 \\
\hline Midwest & 162 & 143 & 140 & 121 & 213 & 152 & 153 & 130 & 141 & 99 & 192 & 141 \\
\hline South & 685 & 621 & 571 & 542 & 513 & 509 & 577 & 528 & 461 & 425 & 427 & 415 \\
\hline West & 300 & 265 & 274 & 281 & 280 & 296 & 192 & 160 & 150 & 168 & 169 & 175 \\
\hline U.S. dependent areas & 112 & 90 & 82 & 67 & 60 & 48 & 33 & 23 & 25 & 14 & 6 & 13 \\
\hline Total & 1,778 & 1,579 & 1,478 & 1,364 & 1,403 & 1,333 & 1,296 & 1,169 & 1,028 & 947 & 1,009 & 952 \\
\hline
\end{tabular}

Note. Data for the year 2016 are preliminary (subject to change) because they are based on only a 6-month reporting delay. Data for the year 2016 should not be used when assessing trends. Numbers less than 12 , and trends based on these numbers, should be interpreted with caution. Data have been statistically adjusted to account for missing transmission category; therefore, values may not sum to column total.

${ }^{\mathrm{a}}$ Data are based on residence at time of diagnosis of HIV infection.

$\mathrm{b}_{\text {Hispanics/Latinos can be of any race. }}$ 
Table 7a. Diagnoses of HIV infection attributed to heterosexual contact, by selected characteristics, 2011-2016-United States

\begin{tabular}{|c|c|c|c|c|c|c|c|c|c|c|c|}
\hline \multicolumn{6}{|c|}{ Male } & \multicolumn{6}{|c|}{ Female } \\
\hline 2011 & 2012 & 2013 & 2014 & 2015 & 2016 & 2011 & 2012 & 2013 & 2014 & 2015 & 2016 \\
\hline No. & No. & No. & No. & No. & No. & No. & No. & No. & No. & No. & No. \\
\hline
\end{tabular}

American Indian/Alaska Native

Age at diagnosis (yr)

\begin{tabular}{|c|c|c|c|c|c|c|c|c|c|c|c|}
\hline $13-14$ & 0 & 0 & 0 & 0 & 0 & 0 & 0 & 0 & 0 & 0 & 0 \\
\hline $15-19$ & 1 & 1 & 0 & 0 & 0 & 0 & 2 & 0 & 1 & 3 & 2 \\
\hline $20-24$ & 2 & 1 & 1 & 0 & 1 & 0 & 1 & 3 & 3 & 1 & 2 \\
\hline $25-29$ & 0 & 0 & 2 & 0 & 0 & 0 & 2 & 2 & 1 & 7 & 7 \\
\hline 30-34 & 1 & 2 & 3 & 3 & 1 & 1 & 4 & 6 & 2 & 2 & 8 \\
\hline $35-39$ & 1 & 0 & 1 & 1 & 1 & 1 & 2 & 3 & 4 & 2 & 5 \\
\hline 40-44 & 3 & 0 & 0 & 1 & 0 & 2 & 3 & 4 & 2 & 2 & 6 \\
\hline $45-49$ & 0 & 4 & 4 & 3 & 3 & 1 & 4 & 4 & 2 & 1 & 3 \\
\hline $50-54$ & 1 & 0 & 1 & 0 & 2 & 0 & 2 & 1 & 6 & 5 & 4 \\
\hline $55-59$ & 0 & 1 & 0 & 1 & 1 & 0 & 3 & 1 & 1 & 3 & 4 \\
\hline $60-64$ & 0 & 2 & 0 & 0 & 0 & 0 & 0 & 3 & 0 & 0 & 0 \\
\hline$\geq 65$ & 0 & 0 & 0 & 0 & 0 & 2 & 0 & 1 & 2 & 0 & 1 \\
\hline
\end{tabular}

Region of residence ${ }^{a}$



Asian

Age at diagnosis (yr)

$13-14$
$15-19$
$20-24$
$25-29$
$30-34$
$35-39$
$40-44$
$45-49$
$50-54$
$55-59$
$60-64$
$\geq 65$

$\begin{array}{rrrrrr}0 & 0 & 0 & 0 & 0 & 0 \\ 0 & 0 & 0 & 1 & 0 & 0 \\ 0 & 0 & 1 & 1 & 0 & 4 \\ 2 & 3 & 7 & 7 & 6 & 3 \\ 6 & 7 & 6 & 13 & 3 & 10 \\ 8 & 7 & 5 & 8 & 19 & 11 \\ 10 & 9 & 9 & 6 & 9 & 6 \\ 7 & 6 & 10 & 6 & 7 & 7 \\ 5 & 7 & 5 & 7 & 5 & 5 \\ 1 & 1 & 4 & 2 & 4 & 3 \\ 5 & 5 & 2 & 1 & 0 & 4 \\ 4 & 2 & 1 & 6 & 4 & 4\end{array}$

$\begin{array}{rr}0 & 0 \\ 6 & 6 \\ 7 & 10 \\ 23 & 24 \\ 13 & 20 \\ 19 & 22 \\ 12 & 14 \\ 7 & 7 \\ 9 & 7 \\ 5 & 4 \\ 6 & 7 \\ 2 & 0\end{array}$

$\begin{array}{rrrr}1 & 0 & 0 & 1 \\ 2 & 1 & 3 & 0 \\ 8 & 10 & 13 & 11 \\ 20 & 20 & 19 & 18 \\ 22 & 20 & 21 & 30 \\ 24 & 23 & 18 & 25 \\ 15 & 18 & 16 & 16 \\ 11 & 9 & 9 & 11 \\ 6 & 8 & 7 & 8 \\ 7 & 8 & 4 & 9 \\ 2 & 5 & 7 & 3 \\ 3 & 1 & 5 & 3\end{array}$

Region of residence ${ }^{a}$

Northeast

Midwest

South

$\begin{array}{rrrrr}7 & 12 & 10 & 15 & 10 \\ 8 & 7 & 6 & 10 & 15 \\ 16 & 13 & 16 & 15 & 23 \\ 18 & 14 & 18 & 16 & 18 \\ 49 & 46 & 50 & 56 & 59\end{array}$

10
8
23
18
59

$\begin{array}{ll}10 & \\ 10 & 20 \\ 22 & 30 \\ 14 & 39 \\ 56 & 108\end{array}$

$\begin{array}{rrr}19 & 20 & 21 \\ 20 & 8 & 19 \\ 30 & 33 & 37 \\ 39 & 56 & 44 \\ 108 & 117 & 12\end{array}$

21
19
37
44
121

22

$19 \quad 35$

West

Total

49


Table 7a. Diagnoses of HIV infection attributed to heterosexual contact, by selected characteristics, 2011-2016-United States (cont)

\begin{tabular}{|c|c|c|c|c|c|c|c|c|c|c|c|c|}
\hline & \multicolumn{6}{|c|}{ Male } & \multicolumn{6}{|c|}{ Female } \\
\hline & 2011 & 2012 & 2013 & 2014 & 2015 & 2016 & 2011 & 2012 & 2013 & 2014 & 2015 & 2016 \\
\hline & No. & No. & No. & No. & No. & No. & No. & No. & No. & No. & No. & No. \\
\hline & \multicolumn{12}{|c|}{ Black/African American } \\
\hline \multicolumn{13}{|c|}{ Age at diagnosis (yr) } \\
\hline $13-14$ & 0 & 0 & 1 & 1 & 1 & 0 & 7 & 13 & 6 & 5 & 8 & 6 \\
\hline $15-19$ & 34 & 36 & 35 & 29 & 34 & 33 & 258 & 213 & 189 & 186 & 144 & 146 \\
\hline $20-24$ & 151 & 166 & 141 & 158 & 161 & 145 & 616 & 491 & 463 & 476 & 473 & 414 \\
\hline $25-29$ & 198 & 175 & 194 & 197 & 249 & 232 & 605 & 604 & 565 & 565 & 531 & 583 \\
\hline $30-34$ & 236 & 234 & 217 & 209 & 207 & 216 & 634 & 648 & 566 & 541 & 530 & 573 \\
\hline $35-39$ & 277 & 233 & 223 & 211 & 215 & 216 & 609 & 552 & 518 & 553 & 543 & 565 \\
\hline $40-44$ & 355 & 305 & 234 & 256 & 248 & 215 & 555 & 549 & 489 & 476 & 437 & 440 \\
\hline $45-49$ & 395 & 350 & 296 & 251 & 244 & 223 & 586 & 564 & 497 & 436 & 404 & 439 \\
\hline $50-54$ & 341 & 295 & 267 & 252 & 255 & 221 & 466 & 442 & 424 & 366 & 394 & 390 \\
\hline $55-59$ & 206 & 212 & 200 & 193 & 208 & 179 & 297 & 309 & 323 & 326 & 298 & 319 \\
\hline $60-64$ & 136 & 123 & 126 & 114 & 122 & 128 & 182 & 173 & 143 & 152 & 180 & 188 \\
\hline$\geq 65$ & 120 & 127 & 126 & 126 & 104 & 117 & 116 & 109 & 122 & 126 & 132 & 126 \\
\hline \multicolumn{13}{|c|}{ Region of residence ${ }^{a}$} \\
\hline Northeast & 487 & 469 & 415 & 420 & 365 & 326 & 867 & 864 & 694 & 761 & 705 & 710 \\
\hline Midwest & 204 & 197 & 215 & 172 & 187 & 189 & 558 & 564 & 537 & 485 & 498 & 508 \\
\hline South & 1,680 & 1,528 & 1,338 & 1,311 & 1,398 & 1,315 & 3,248 & 2,984 & 2,845 & 2,714 & 2,612 & 2,658 \\
\hline West & 78 & 63 & 91 & 94 & 98 & 96 & 258 & 254 & 231 & 248 & 260 & 313 \\
\hline Total & 2,449 & 2,257 & 2,059 & 1,997 & 2,048 & 1,926 & 4,931 & 4,666 & 4,307 & 4,208 & 4,075 & 4,189 \\
\hline & \multicolumn{12}{|c|}{ Hispanic/Latino ${ }^{b}$} \\
\hline \multicolumn{13}{|c|}{ Age at diagnosis (yr) } \\
\hline $13-14$ & 0 & 0 & 0 & 0 & 0 & 0 & 5 & 0 & 2 & 1 & 0 & 2 \\
\hline $15-19$ & 9 & 6 & 7 & 3 & 6 & 5 & 58 & 45 & 43 & 45 & 36 & 26 \\
\hline $20-24$ & 36 & 41 & 37 & 39 & 32 & 50 & 132 & 108 & 91 & 124 & 120 & 96 \\
\hline $25-29$ & 74 & 53 & 79 & 62 & 53 & 63 & 145 & 144 & 150 & 136 & 137 & 132 \\
\hline 30-34 & 111 & 99 & 113 & 97 & 85 & 81 & 177 & 136 & 138 & 162 & 137 & 159 \\
\hline $35-39$ & 114 & 81 & 98 & 105 & 97 & 77 & 152 & 137 & 133 & 161 & 149 & 128 \\
\hline $40-44$ & 100 & 91 & 83 & 101 & 81 & 82 & 146 & 130 & 126 & 126 & 127 & 124 \\
\hline $45-49$ & 85 & 79 & 74 & 86 & 74 & 82 & 143 & 123 & 122 & 116 & 111 & 107 \\
\hline $50-54$ & 63 & 65 & 60 & 71 & 63 & 52 & 100 & 88 & 115 & 93 & 110 & 99 \\
\hline $55-59$ & 41 & 42 & 53 & 43 & 44 & 38 & 64 & 64 & 62 & 77 & 62 & 72 \\
\hline $60-64$ & 30 & 26 & 22 & 22 & 25 & 25 & 40 & 35 & 39 & 40 & 37 & 42 \\
\hline$\geq 65$ & 30 & 29 & 21 & 21 & 36 & 24 & 30 & 44 & 29 & 29 & 28 & 38 \\
\hline \multicolumn{13}{|c|}{ Region of residence $^{a}$} \\
\hline Northeast & 217 & 190 & 176 & 172 & 159 & 149 & 419 & 362 & 353 & 347 & 316 & 338 \\
\hline Midwest & 51 & 37 & 39 & 35 & 39 & 36 & 86 & 62 & 63 & 69 & 49 & 52 \\
\hline South & 337 & 291 & 324 & 335 & 307 & 297 & 471 & 398 & 407 & 424 & 463 & 432 \\
\hline West & 87 & 94 & 107 & 107 & 90 & 99 & 213 & 232 & 227 & 271 & 224 & 203 \\
\hline Total & 692 & 612 & 646 & 649 & 595 & 581 & 1,189 & 1,054 & 1,050 & 1,111 & 1,052 & 1,025 \\
\hline
\end{tabular}


Table 7a. Diagnoses of HIV infection attributed to heterosexual contact, by selected characteristics, 2011-2016-United States (cont)

\begin{tabular}{|c|c|c|c|c|c|c|c|c|c|c|c|}
\hline \multicolumn{6}{|c|}{ Male } & \multicolumn{6}{|c|}{ Female } \\
\hline 2011 & 2012 & 2013 & 2014 & 2015 & 2016 & 2011 & 2012 & 2013 & 2014 & 2015 & 2016 \\
\hline No. & No. & No. & No. & No. & No. & No. & No. & No. & No. & No. & No. \\
\hline
\end{tabular}

Native Hawaiian/Other Pacific Islander

Age at diagnosis (yr)

\begin{tabular}{|c|c|c|c|c|c|c|c|c|c|c|c|}
\hline $13-14$ & 0 & 0 & 0 & 0 & 0 & 0 & 0 & 0 & 0 & 0 & 0 \\
\hline $15-19$ & 0 & 0 & 0 & 0 & 0 & 0 & 0 & 0 & 0 & 1 & 1 \\
\hline 20-24 & 0 & 0 & 0 & 0 & 0 & 0 & 0 & 0 & 4 & 1 & 2 \\
\hline $25-29$ & 0 & 0 & 0 & 0 & 1 & 0 & 1 & 1 & 0 & 0 & 0 \\
\hline $30-34$ & 0 & 0 & 0 & 0 & 0 & 0 & 0 & 3 & 1 & 1 & 1 \\
\hline $35-39$ & 1 & 0 & 0 & 0 & 2 & 1 & 1 & 1 & 0 & 1 & 0 \\
\hline $40-44$ & 0 & 0 & 0 & 1 & 0 & 0 & 1 & 0 & 1 & 1 & 1 \\
\hline $45-49$ & 0 & 0 & 0 & 0 & 1 & 1 & 2 & 0 & 1 & 0 & 0 \\
\hline $50-54$ & 0 & 0 & 0 & 0 & 1 & 0 & 0 & 1 & 3 & 1 & 1 \\
\hline $55-59$ & 0 & 0 & 0 & 0 & 1 & 0 & 2 & 0 & 0 & 1 & 1 \\
\hline $60-64$ & 0 & 0 & 0 & 0 & 0 & 0 & 0 & 0 & 1 & 1 & 0 \\
\hline$\geq 65$ & 0 & 1 & 0 & 0 & 0 & 0 & 0 & 0 & 0 & 0 & 0 \\
\hline
\end{tabular}

Region of residence ${ }^{a}$

Northeast

Midwest

South

West

0

0

0
0
0
0
0

Total

$\begin{array}{lll}0 & 0 & 0 \\ 1 & 2 & 0 \\ 1 & 2 & 0\end{array}$

$\begin{array}{ll}1 & 0 \\ 0 & 0 \\ 0 & 1 \\ 0 & 4 \\ 1 & 5\end{array}$

$\begin{array}{lll}0 & 0 & 1 \\ 1 & 0 & 0 \\ 2 & 1 & 1 \\ 0 & 6 & 4 \\ 3 & 7 & 6\end{array}$

\section{White}

Age at diagnosis (yr)

13-14

15-19

20-24

25-29

30-34

35-39

40-44

45-49

50-54

55-59

60-64

$\geq 65$

Region of residence ${ }^{a}$

Northeast

Midwest

South

West

Total

$\begin{array}{rrr}0 & 0 & 0 \\ 2 & 2 & 3 \\ 17 & 19 & 13 \\ 38 & 41 & 38 \\ 28 & 36 & 31 \\ 33 & 38 & 47 \\ 66 & 70 & 75 \\ 71 & 70 & 64 \\ 66 & 63 & 56 \\ 48 & 42 & 54 \\ 40 & 26 & 32 \\ 39 & 30 & 43\end{array}$

\section{0}

$109 \quad 89$

$64 \quad 70$

90

$$
64
$$

0
3
16
40
42
29
57
61
74
41
29
38

$\begin{array}{rr}0 & \\ 3 & \\ 13 & 29 \\ 41 & 36 \\ 38 & 38 \\ 37 & 42 \\ 50 & 4 \\ 61 & 45 \\ 39 & 74 \\ 47 & 50 \\ 35 & 28 \\ 44 & 35\end{array}$

$3 \quad 40$

29

36

38

42

44

45

74

50

28

93

$74 \quad 74$

$52 \quad 45$

$221 \quad 235$

202

60

70

$\begin{array}{rr}3 & 2 \\ 40 & 34 \\ 105 & 113 \\ 130 & 124 \\ 127 & 112 \\ 121 & 104 \\ 142 & 142 \\ 125 & 118 \\ 108 & 99 \\ 55 & 69 \\ 38 & 30 \\ 19 & 22\end{array}$

3

$$
20
$$

$$
106
$$$$
108
$$$$
116
$$$$
111
$$$$
96
$$$$
111
$$$$
93
$$$$
71
$$$$
53
$$$$
26
$$

$\begin{array}{rr}148 & 141 \\ 183 & 163 \\ 536 & 498 \\ 147 & 168 \\ \mathbf{1 , 0 1 4} & \mathbf{9 7 0}\end{array}$

\section{6}

$$
154
$$$$
459
$$$$
\begin{aligned}
& 459 \\
& 154
\end{aligned}
$$$$
\begin{aligned}
& 154 \\
& 913
\end{aligned}
$$

407

424

$447 \quad 437$

456

\begin{abstract}
430
\end{abstract}

\begin{abstract}
1,01
\end{abstract}

\begin{abstract}
970
\end{abstract}

$\begin{array}{rrr}3 & 0 & 0 \\ 30 & 23 & 32 \\ 119 & 99 & 106 \\ 127 & 127 & 146 \\ 134 & 124 & 143 \\ 120 & 107 & 122 \\ 112 & 106 & 104 \\ 105 & 116 & 109 \\ 110 & 111 & 106 \\ 82 & 90 & 88 \\ 30 & 32 & 55 \\ 15 & 27 & 23 \\ & & \\ 148 & 132 & 148 \\ 156 & 163 & 165 \\ 513 & 519 & 519 \\ 171 & 148 & 200 \\ 988 & 962 & 1,032\end{array}$


Table 7a. Diagnoses of HIV infection attributed to heterosexual contact, by selected characteristics, 2011-2016-United States (cont)

\begin{tabular}{|c|c|c|c|c|c|c|c|c|c|c|c|c|}
\hline & \multicolumn{6}{|c|}{ Male } & \multicolumn{6}{|c|}{ Female } \\
\hline & 2011 & 2012 & 2013 & 2014 & 2015 & 2016 & 2011 & 2012 & 2013 & 2014 & 2015 & 2016 \\
\hline & No. & No. & No. & No. & No. & No. & No. & No. & No. & No. & No. & No. \\
\hline & \multicolumn{12}{|c|}{ Multiple races } \\
\hline \multicolumn{13}{|c|}{ Age at diagnosis (yr) } \\
\hline $13-14$ & 0 & 0 & 0 & 0 & 0 & 0 & 0 & 1 & 0 & 0 & 0 & 1 \\
\hline $15-19$ & 1 & 2 & 2 & 1 & 0 & 0 & 18 & 14 & 15 & 10 & 7 & 6 \\
\hline 20-24 & 8 & 7 & 8 & 4 & 9 & 4 & 31 & 35 & 27 & 27 & 13 & 16 \\
\hline $25-29$ & 8 & 9 & 11 & 7 & 10 & 6 & 25 & 23 & 28 & 24 & 19 & 12 \\
\hline $30-34$ & 11 & 9 & 11 & 8 & 8 & 2 & 31 & 31 & 27 & 25 & 25 & 19 \\
\hline $35-39$ & 10 & 16 & 9 & 16 & 8 & 6 & 26 & 24 & 27 & 23 & 21 & 17 \\
\hline $40-44$ & 19 & 16 & 14 & 9 & 3 & 5 & 31 & 28 & 19 & 29 & 8 & 9 \\
\hline $45-49$ & 14 & 11 & 14 & 17 & 4 & 6 & 31 & 25 & 24 & 16 & 16 & 17 \\
\hline $50-54$ & 12 & 8 & 11 & 4 & 7 & 3 & 22 & 15 & 18 & 14 & 16 & 9 \\
\hline $55-59$ & 8 & 8 & 8 & 9 & 5 & 4 & 17 & 16 & 15 & 9 & 12 & 6 \\
\hline $60-64$ & 3 & 7 & 7 & 2 & 1 & 3 & 8 & 11 & 10 & 9 & 5 & 3 \\
\hline$\geq 65$ & 5 & 2 & 5 & 4 & 8 & 1 & 8 & 8 & 7 & 4 & 3 & 7 \\
\hline \multicolumn{13}{|c|}{ Region of residence ${ }^{a}$} \\
\hline Northeast & 39 & 35 & 37 & 26 & 17 & 15 & 73 & 67 & 64 & 45 & 42 & 25 \\
\hline Midwest & 7 & 13 & 8 & 8 & 7 & 5 & 27 & 27 & 33 & 22 & 15 & 26 \\
\hline South & 48 & 38 & 47 & 35 & 31 & 17 & 129 & 120 & 104 & 105 & 75 & 61 \\
\hline West & 3 & 10 & 7 & 12 & 7 & 3 & 19 & 18 & 16 & 17 & 14 & 8 \\
\hline \multirow[t]{2}{*}{ Total } & 97 & 96 & 99 & 81 & 62 & 40 & 248 & 232 & 217 & 189 & 146 & 120 \\
\hline & \multicolumn{12}{|c|}{ Total } \\
\hline \multicolumn{13}{|c|}{ Age at diagnosis (yr) } \\
\hline $13-14$ & 0 & 0 & 1 & 1 & 1 & 0 & 14 & 16 & 12 & 8 & 8 & 10 \\
\hline $15-19$ & 47 & 47 & 48 & 36 & 43 & 42 & 381 & 311 & 269 & 276 & 216 & 210 \\
\hline $20-24$ & 214 & 235 & 201 & 218 & 217 & 232 & 892 & 759 & 702 & 757 & 720 & 645 \\
\hline $25-29$ & 319 & 280 & 330 & 313 & 360 & 340 & 930 & 923 & 872 & 878 & 839 & 895 \\
\hline $30-34$ & 393 & 386 & 381 & 373 & 342 & 348 & 987 & 954 & 871 & 884 & 847 & 929 \\
\hline $35-39$ & 444 & 376 & 383 & 371 & 379 & 355 & 929 & 844 & 815 & 884 & 842 & 865 \\
\hline $40-44$ & 553 & 492 & 415 & 429 & 391 & 355 & 889 & 867 & 749 & 765 & 700 & 700 \\
\hline $45-49$ & 571 & 519 & 462 & 424 & 394 & 364 & 897 & 841 & 769 & 684 & 658 & 685 \\
\hline $50-54$ & 489 & 439 & 399 & 408 & 373 & 354 & 707 & 652 & 664 & 598 & 642 & 616 \\
\hline $55-59$ & 303 & 307 & 319 & 288 & 309 & 274 & 442 & 463 & 479 & 505 & 471 & 495 \\
\hline $60-64$ & 213 & 188 & 188 & 168 & 183 & 188 & 272 & 258 & 247 & 238 & 262 & 292 \\
\hline$\geq 65$ & 198 & 192 & 196 & 195 & 196 & 184 & 176 & 183 & 189 & 174 & 196 & 198 \\
\hline \multicolumn{13}{|c|}{ Region of residence ${ }^{a}$} \\
\hline Northeast & 859 & 797 & 731 & 729 & 627 & 574 & 1,527 & 1,457 & 1,278 & 1,324 & 1,214 & 1,258 \\
\hline Midwest & 334 & 326 & 337 & 280 & 295 & 288 & 875 & 831 & 811 & 749 & 751 & 764 \\
\hline South & 2,296 & 2,072 & 1,946 & 1,899 & 1,984 & 1,888 & 4,420 & 4,039 & 3,863 & 3,803 & 3,734 & 3,727 \\
\hline West & 255 & 267 & 307 & 315 & 282 & 287 & 695 & 744 & 688 & 777 & 703 & 792 \\
\hline Total & 3,745 & 3,461 & 3,323 & 3,223 & 3,187 & 3,037 & 7,517 & 7,071 & 6,640 & 6,653 & 6,401 & 6,541 \\
\hline
\end{tabular}

Note. Data for the year 2016 are preliminary (subject to change) because they are based on only a 6-month reporting delay. Data for the year 2016 should not be used when assessing trends. Numbers less than 12 , and trends based on these numbers, should be interpreted with caution. Data have been statistically adjusted to account for missing transmission category; therefore, values may not sum to column total. Data include persons with HIV infection attributed to heterosexual contact with a person known to have, or to be at high risk for, HIV infection.

a Data are based on residence at time of diagnosis of HIV infection.

$\mathrm{b}_{\text {Hispanics/Latinos can be of any race. }}$ 
Table 7b. Diagnoses of HIV infection attributed to heterosexual contact, by selected characteristics, 2011-2016-United States and 6 dependent areas



\section{American Indian/Alaska Native}

\section{Age at diagnosis (yr)}

13-14

$15-19$

$20-24$

25-29

30-34

35-39

40-44

$45-49$

$50-54$

55-59

60-64

$\geq 65$

Region of residence ${ }^{a}$

Northeast

Midwest

South

West

U.S. dependent areas

Total

0
1
2
0
1
1
3
0
1
0
0
0

0
1
1
0
2
0
0
4
0
1
2
0

0
0
0
0
3
1
1
3
0
1
0
0

0
0
1
0
1
1
0
3
2
1
0
0

0
0
0
0
1
1
2
1
0
0
0
2

0
2
1
2
4
2
3
4
2
3
0
0

0
0
3
2
6
3
4
4
1
1
3
1

$\begin{array}{llll}0 & 0 & 0 & 0 \\ 1 & 3 & 2 & 0 \\ 3 & 1 & 2 & 1 \\ 1 & 7 & 7 & 5 \\ 2 & 2 & 8 & 3 \\ 4 & 2 & 5 & 6 \\ 2 & 2 & 6 & 8 \\ 2 & 1 & 3 & 3 \\ 6 & 5 & 4 & 1 \\ 1 & 3 & 4 & 2 \\ 0 & 0 & 0 & 2 \\ 2 & 0 & 1 & 1\end{array}$

\section{Age at diagnosis (yr)}

$13-14$
$15-19$
$20-24$
$25-29$
$30-34$
$35-39$
$40-44$
$45-49$
$50-54$
$55-59$
$60-64$
$\geq 65$

$\begin{array}{rl}0 & 0 \\ 0 & 0 \\ 0 & 0 \\ 2 & 3 \\ 6 & 7 \\ 8 & 7 \\ 10 & 9 \\ 7 & 6 \\ 5 & 7 \\ 1 & 1 \\ 5 & 5 \\ 4 & 2\end{array}$

Region of residence ${ }^{a}$

Northeast

Midwest

South

West

U.S. dependent areas

Total

$\begin{array}{rrrrrr}7 & 12 & 10 & 15 & 10 & 10 \\ 8 & 7 & 6 & 10 & 8 & 10 \\ 16 & 13 & 16 & 15 & 23 & 22 \\ 18 & 14 & 18 & 16 & 18 & 14 \\ 0 & 0 & 0 & 0 & 0 & 0 \\ 49 & 46 & 50 & 56 & 59 & 56\end{array}$

0
0
1
7
6
5
9
10
5
4
2
1

$\begin{array}{rr}0 & 0 \\ 1 & 0 \\ 1 & 0 \\ 7 & 6 \\ 13 & 3 \\ 8 & 19 \\ 6 & 9 \\ 6 & 7 \\ 7 & 5 \\ 2 & 4 \\ 1 & 0 \\ 6 & 4\end{array}$

$\begin{array}{rr}0 & 0 \\ 0 & 6 \\ 4 & 7 \\ 3 & 23 \\ 10 & 14 \\ 11 & 19 \\ 6 & 12 \\ 7 & 7 \\ 5 & 9 \\ 3 & 5 \\ 4 & 6 \\ 4 & 2\end{array}$

0
6
10
24
20
22
14
7
7
4
7
0

1
2
8
20
22
24
15
11
6
7
2
3

$\begin{array}{rrr}0 & 0 & 1 \\ 1 & 3 & 0 \\ 10 & 13 & 11 \\ 20 & 19 & 18 \\ 20 & 21 & 30 \\ 23 & 18 & 25 \\ 18 & 16 & 16 \\ 9 & 9 & 11 \\ 8 & 7 & 8 \\ 8 & 4 & 9 \\ 5 & 7 & 3 \\ 1 & 5 & 3\end{array}$

$\begin{array}{rrrrrr}19 & 20 & 21 & 22 & 19 & 35 \\ 20 & 8 & 19 & 13 & 16 & 12 \\ 30 & 33 & 37 & 37 & 45 & 44 \\ 39 & 56 & 44 & 52 & 41 & 46 \\ 1 & 0 & 0 & 0 & 0 & 0 \\ 109 & 117 & 121 & 124 & 121 & 137\end{array}$


Table 7b. Diagnoses of HIV infection attributed to heterosexual contact, by selected characteristics, 2011-2016-United States and 6 dependent areas (cont)

\begin{tabular}{|c|c|c|c|c|c|c|c|c|c|c|c|}
\hline \multicolumn{6}{|c|}{ Male } & \multicolumn{6}{|c|}{ Female } \\
\hline 2011 & 2012 & 2013 & 2014 & 2015 & 2016 & 2011 & 2012 & 2013 & 2014 & 2015 & 2016 \\
\hline No. & No. & No. & No. & No. & No. & No. & No. & No. & No. & No. & No. \\
\hline
\end{tabular}

Black/African American

Age at diagnosis (yr)

13-14

$15-19$

20-24

$25-29$

30-34

35-39

40-44

45-49

50-54

55-59

60-64

$\geq 65$

Region of residence ${ }^{a}$

Northeast

Midwest

South

West

U.S. dependent areas

Total

454

\section{0}

$34 \quad 36$

$152 \quad 166$

$198 \quad 175$

$236 \quad 234$

$278 \quad 233$

$355 \quad 305$

$395 \quad 350$

$341 \quad 295$

$206 \quad 212$

$138 \quad 123$

$120 \quad 127$

$\begin{array}{rrr}1 & 1 & 1 \\ 35 & 29 & 34 \\ 141 & 158 & 161 \\ 195 & 197 & 249 \\ 217 & 209 & 207 \\ 223 & 211 & 215 \\ 234 & 256 & 248 \\ 296 & 251 & 244 \\ 269 & 255 & 255 \\ 200 & 193 & 208 \\ 127 & 115 & 122 \\ 126 & 127 & 104\end{array}$

$\begin{array}{rr}0 & 7 \\ 33 & 258 \\ 145 & 616 \\ 232 & 605 \\ 216 & 634 \\ 216 & 612 \\ 215 & 556 \\ 224 & 587 \\ 221 & 466 \\ 179 & 297 \\ 128 & 182 \\ 117 & 116\end{array}$

$$
\begin{array}{r}
13 \\
213
\end{array}
$$

6
189

5
186

$8 \quad 6$

616

491

464

$\begin{array}{ll}604 & 566 \\ 649 & 566\end{array}$

$552 \quad 518$

$550 \quad 489$

$564 \quad 499$

442

424

325

325
144

173

123

109

\begin{tabular}{|c|c|c|c|c|c|c|c|c|c|c|c|}
\hline 487 & 469 & 415 & 420 & 365 & 326 & 867 & 864 & 694 & 761 & 705 & 710 \\
\hline 204 & 197 & 215 & 172 & 187 & 189 & 558 & 564 & 537 & 485 & 498 & 508 \\
\hline 1,680 & 1,528 & 1,338 & 1,311 & 1,398 & 1,315 & 3,248 & 2,984 & 2,845 & 2,714 & 2,612 & 2,658 \\
\hline 78 & 63 & 91 & 94 & 98 & 96 & 258 & 254 & 231 & 248 & 260 & 313 \\
\hline 5 & 1 & 4 & 5 & 0 & 2 & 5 & 2 & 7 & 5 & 4 & 0 \\
\hline 2,454 & 2,258 & 2,063 & 2,002 & 2,048 & 1,928 & 4,936 & 4,668 & 4,314 & 4,213 & 4,079 & 4,189 \\
\hline
\end{tabular}

Hispanic/Latino ${ }^{b}$

Age at diagnosis (yr)

\begin{tabular}{|c|c|c|c|c|c|c|c|c|c|c|c|c|}
\hline $13-14$ & 0 & 0 & 0 & 0 & 0 & 0 & 5 & 1 & 3 & 1 & 0 & 2 \\
\hline $15-19$ & 10 & 7 & 9 & 3 & 6 & 5 & 65 & 50 & 46 & 53 & 39 & 29 \\
\hline 20-24 & 41 & 45 & 44 & 46 & 39 & 52 & 145 & 122 & 103 & 134 & 129 & 100 \\
\hline $25-29$ & 88 & 61 & 85 & 73 & 59 & 73 & 162 & 157 & 159 & 143 & 146 & 138 \\
\hline $30-34$ & 122 & 113 & 122 & 105 & 96 & 89 & 198 & 152 & 150 & 176 & 143 & 167 \\
\hline $35-39$ & 122 & 94 & 108 & 113 & 105 & 85 & 163 & 157 & 149 & 180 & 158 & 143 \\
\hline $40-44$ & 123 & 114 & 91 & 113 & 87 & 95 & 173 & 152 & 140 & 147 & 140 & 137 \\
\hline $45-49$ & 111 & 103 & 96 & 98 & 89 & 100 & 169 & 150 & 142 & 131 & 126 & 122 \\
\hline $50-54$ & 87 & 84 & 75 & 84 & 77 & 65 & 121 & 110 & 137 & 104 & 122 & 109 \\
\hline $55-59$ & 55 & 53 & 62 & 56 & 50 & 47 & 76 & 75 & 77 & 88 & 74 & 87 \\
\hline 60-64 & 40 & 32 & 32 & 36 & 28 & 34 & 45 & 43 & 44 & 44 & 38 & 49 \\
\hline$\geq 65$ & 38 & 44 & 34 & 29 & 46 & 35 & 41 & 60 & 33 & 34 & 35 & 40 \\
\hline \multicolumn{13}{|l|}{ Region of residence ${ }^{a}$} \\
\hline Northeast & 217 & 190 & 176 & 172 & 159 & 149 & 419 & 362 & 353 & 347 & 316 & 338 \\
\hline Midwest & 51 & 37 & 39 & 35 & 39 & 36 & 86 & 62 & 63 & 69 & 49 & 52 \\
\hline South & 337 & 291 & 324 & 335 & 307 & 297 & 471 & 398 & 407 & 424 & 463 & 432 \\
\hline West & 87 & 94 & 107 & 107 & 90 & 99 & 213 & 232 & 227 & 271 & 224 & 203 \\
\hline U.S. dependent areas & 144 & 136 & 112 & 106 & 86 & 99 & 172 & 175 & 132 & 124 & 97 & 96 \\
\hline Total & 836 & 748 & 758 & 755 & 681 & 680 & 1,361 & 1,229 & 1,182 & 1,235 & 1,149 & 1,121 \\
\hline
\end{tabular}


Table 7b. Diagnoses of HIV infection attributed to heterosexual contact, by selected characteristics, 2011-2016-United States and 6 dependent areas (cont)

\begin{tabular}{|c|c|c|c|c|c|c|c|c|c|c|c|}
\hline \multicolumn{6}{|c|}{ Male } & \multicolumn{6}{|c|}{ Female } \\
\hline 2011 & 2012 & 2013 & 2014 & 2015 & 2016 & 2011 & 2012 & 2013 & 2014 & 2015 & 2016 \\
\hline No. & No. & No. & No. & No. & No. & No. & No. & No. & No. & No. & No. \\
\hline
\end{tabular}

Age at diagnosis (yr)

13-14

$15-19$

20-24

$25-29$

$30-34$

$35-39$

40-44

$45-49$

$50-54$

$55-59$

60-64

$\geq 65$

\section{Region of residence ${ }^{a}$}

Northeast

Midwest

South

West

U.S. dependent areas

Total

Age at diagnosis (yr)

\begin{tabular}{|c|c|c|c|c|c|c|c|c|c|c|c|c|}
\hline $13-14$ & 0 & 0 & 0 & 0 & 0 & 0 & 3 & 2 & 3 & 3 & 0 & 0 \\
\hline $15-19$ & 2 & 2 & 3 & 3 & 3 & 3 & 40 & 34 & 20 & 30 & 23 & 32 \\
\hline $20-24$ & 17 & 19 & 13 & 16 & 13 & 29 & 105 & 113 & 106 & 119 & 99 & 107 \\
\hline $25-29$ & 38 & 41 & 38 & 40 & 41 & 36 & 130 & 124 & 108 & 127 & 127 & 146 \\
\hline $30-34$ & 28 & 36 & 31 & 42 & 38 & 38 & 127 & 112 & 116 & 134 & 124 & 143 \\
\hline $35-39$ & 33 & 38 & 47 & 29 & 37 & 42 & 121 & 104 & 111 & 120 & 107 & 122 \\
\hline $40-44$ & 67 & 70 & 75 & 57 & 50 & 44 & 142 & 142 & 96 & 112 & 106 & 104 \\
\hline $45-49$ & 71 & 70 & 64 & 61 & 61 & 45 & 125 & 118 & 111 & 105 & 116 & 109 \\
\hline $50-54$ & 66 & 63 & 56 & 74 & 39 & 74 & 108 & 99 & 93 & 110 & 111 & 106 \\
\hline $55-59$ & 48 & 42 & 54 & 41 & 47 & 50 & 55 & 69 & 71 & 82 & 90 & 88 \\
\hline $60-64$ & 40 & 26 & 32 & 29 & 35 & 28 & 38 & 30 & 54 & 30 & 32 & 55 \\
\hline$\geq 65$ & 39 & 30 & 43 & 38 & 44 & 35 & 19 & 22 & 26 & 16 & 27 & 23 \\
\hline \multicolumn{13}{|l|}{ Region of residence ${ }^{a}$} \\
\hline Northeast & 109 & 89 & 90 & 93 & 74 & 74 & 148 & 141 & 146 & 148 & 132 & 148 \\
\hline Midwest & 64 & 70 & 64 & 55 & 52 & 45 & 183 & 163 & 154 & 156 & 163 & 165 \\
\hline South & 214 & 201 & 221 & 202 & 221 & 235 & 536 & 498 & 459 & 513 & 519 & 519 \\
\hline West & 60 & 77 & 81 & 80 & 60 & 70 & 147 & 168 & 154 & 171 & 148 & 200 \\
\hline U.S. dependent areas & 1 & 0 & 0 & 0 & 0 & 0 & 0 & 0 & 1 & 1 & 0 & 1 \\
\hline Total & 448 & 437 & 456 & 430 & 407 & 424 & 1,014 & 970 & 914 & 989 & 962 & 1,033 \\
\hline
\end{tabular}

$\begin{array}{llllllllllll}0 & 0 & 0 & 0 & 0 & 0 & 0 & 0 & 0 & 0 & 0 & 0 \\ 0 & 0 & 0 & 0 & 0 & 0 & 0 & 0 & 0 & 1 & 1 & 0 \\ 0 & 0 & 0 & 0 & 0 & 0 & 0 & 0 & 4 & 1 & 2 & 1 \\ 0 & 0 & 0 & 0 & 1 & 0 & 1 & 1 & 0 & 0 & 0 & 0 \\ 0 & 0 & 0 & 0 & 0 & 0 & 0 & 3 & 1 & 1 & 1 & 2 \\ 1 & 0 & 0 & 0 & 2 & 1 & 1 & 1 & 0 & 1 & 0 & 2 \\ 0 & 0 & 0 & 1 & 0 & 0 & 1 & 0 & 1 & 1 & 1 & 0 \\ 0 & 0 & 0 & 0 & 1 & 1 & 2 & 0 & 1 & 0 & 0 & 0 \\ 0 & 0 & 0 & 0 & 2 & 0 & 0 & 1 & 3 & 1 & 1 & \\ 0 & 0 & 0 & 0 & 1 & 0 & 2 & 0 & 0 & 1 & 1 & \\ 0 & 0 & 0 & 0 & 0 & 0 & 0 & 0 & 1 & 1 & 0 & 0 \\ 0 & 1 & 0 & 0 & 0 & 0 & 0 & 0 & 0 & 0 & 0 & 0\end{array}$

Native Hawaiian/Other Pacific Islander 
Table 7b. Diagnoses of HIV infection attributed to heterosexual contact, by selected characteristics, 2011-2016-United States and 6 dependent areas (cont)

\begin{tabular}{|c|c|c|c|c|c|c|c|c|c|c|c|}
\hline \multicolumn{6}{|c|}{ Male } & \multicolumn{6}{|c|}{ Female } \\
\hline 2011 & 2012 & 2013 & 2014 & 2015 & 2016 & 2011 & 2012 & 2013 & 2014 & 2015 & 2016 \\
\hline No. & No. & No. & No. & No. & No. & No. & No. & No. & No. & No. & No. \\
\hline
\end{tabular}

Age at diagnosis (yr)

\begin{tabular}{|c|c|c|c|c|c|c|c|c|c|c|c|c|}
\hline $13-14$ & 0 & 0 & 0 & 0 & 0 & 0 & 0 & 1 & 0 & 0 & 0 & 1 \\
\hline $15-19$ & 1 & 2 & 2 & 1 & 0 & 0 & 18 & 14 & 15 & 10 & 7 & 6 \\
\hline 20-24 & 8 & 7 & 8 & 4 & 9 & 4 & 31 & 35 & 27 & 27 & 14 & 16 \\
\hline 25-29 & 8 & 9 & 11 & 7 & 10 & 6 & 25 & 23 & 28 & 24 & 19 & 12 \\
\hline 30-34 & 11 & 9 & 11 & 8 & 8 & 2 & 31 & 31 & 27 & 25 & 25 & 19 \\
\hline 35-39 & 10 & 16 & 9 & 16 & 8 & 6 & 27 & 24 & 27 & 23 & 21 & 17 \\
\hline $40-44$ & 19 & 16 & 14 & 9 & 3 & 5 & 31 & 28 & 19 & 29 & 8 & 9 \\
\hline $45-49$ & 14 & 11 & 14 & 17 & 4 & 6 & 31 & 25 & 24 & 16 & 16 & 17 \\
\hline $50-54$ & 12 & 8 & 11 & 4 & 7 & 3 & 22 & 15 & 18 & 14 & 16 & 9 \\
\hline $55-59$ & 8 & 8 & 8 & 9 & 5 & 4 & 17 & 16 & 15 & 9 & 12 & 6 \\
\hline $60-64$ & 3 & 7 & 7 & 2 & 1 & 3 & 8 & 11 & 10 & 9 & 5 & 3 \\
\hline$\geq 65$ & 5 & 2 & 5 & 4 & 8 & 1 & 8 & 8 & 7 & 4 & 3 & 7 \\
\hline \multicolumn{13}{|l|}{ Region of residence ${ }^{a}$} \\
\hline Northeast & 39 & 35 & 37 & 26 & 17 & 15 & 73 & 67 & 64 & 45 & 42 & 25 \\
\hline Midwest & 7 & 13 & 8 & 8 & 7 & 5 & 27 & 27 & 33 & 22 & 15 & 26 \\
\hline South & 48 & 38 & 47 & 35 & 31 & 17 & 129 & 120 & 104 & 105 & 75 & 61 \\
\hline West & 3 & 10 & 7 & 12 & 7 & 3 & 19 & 18 & 16 & 17 & 14 & 8 \\
\hline U.S. dependent areas & 0 & 0 & 0 & 0 & 0 & 0 & 1 & 0 & 0 & 0 & 1 & 0 \\
\hline \multirow[t]{2}{*}{ Total } & 97 & 96 & 99 & 81 & 62 & 40 & 249 & 232 & 217 & 189 & 147 & 120 \\
\hline & \multicolumn{12}{|c|}{ Total } \\
\hline \multicolumn{13}{|l|}{ Age at diagnosis (yr) } \\
\hline $13-14$ & 0 & 0 & 1 & 1 & 1 & 0 & 14 & 17 & 13 & 8 & 8 & 10 \\
\hline $15-19$ & 48 & 48 & 50 & 36 & 43 & 42 & 388 & 316 & 272 & 284 & 219 & 213 \\
\hline 20-24 & 220 & 239 & 209 & 225 & 224 & 234 & 905 & 773 & 714 & 767 & 730 & 650 \\
\hline $25-29$ & 333 & 289 & 337 & 323 & 367 & 350 & 947 & 936 & 882 & 885 & 850 & 901 \\
\hline $30-34$ & 404 & 400 & 390 & 381 & 353 & 356 & 1,009 & 972 & 883 & 899 & 853 & 937 \\
\hline $35-39$ & 453 & 388 & 393 & 378 & 388 & 363 & 944 & 864 & 831 & 904 & 851 & 880 \\
\hline $40-44$ & 577 & 515 & 422 & 442 & 397 & 368 & 918 & 890 & 763 & 786 & 713 & 713 \\
\hline $45-49$ & 598 & 543 & 484 & 436 & 409 & 384 & 924 & 867 & 791 & 700 & 673 & 700 \\
\hline $50-54$ & 513 & 457 & 416 & 424 & 388 & 367 & 728 & 674 & 686 & 610 & 655 & 626 \\
\hline $55-59$ & 318 & 318 & 328 & 302 & 314 & 284 & 454 & 474 & 496 & 517 & 483 & 510 \\
\hline $60-64$ & 224 & 194 & 199 & 182 & 187 & 197 & 278 & 266 & 254 & 242 & 263 & 299 \\
\hline$\geq 65$ & 206 & 206 & 210 & 204 & 206 & 194 & 187 & 199 & 194 & 180 & 205 & 200 \\
\hline \multicolumn{13}{|l|}{ Region of residence ${ }^{a}$} \\
\hline Northeast & 859 & 797 & 731 & 729 & 627 & 574 & 1,527 & 1,457 & 1,278 & 1,324 & 1,214 & 1,258 \\
\hline Midwest & 334 & 326 & 337 & 280 & 295 & 288 & 875 & 831 & 811 & 749 & 751 & 764 \\
\hline South & 2,296 & 2,072 & 1,946 & 1,899 & 1,984 & 1,888 & 4,420 & 4,039 & 3,863 & 3,803 & 3,734 & 3,727 \\
\hline West & 255 & 267 & 307 & 315 & 282 & 287 & 695 & 744 & 688 & 777 & 703 & 792 \\
\hline U.S. dependent areas & 150 & 137 & 117 & 111 & 87 & 101 & 178 & 177 & 140 & 130 & 101 & 97 \\
\hline Total & 3,894 & 3,598 & 3,439 & 3,334 & 3,274 & 3,138 & 7,696 & 7,248 & 6,781 & 6,782 & 6,502 & 6,638 \\
\hline
\end{tabular}

Note. Data for the year 2016 are preliminary (subject to change) because they are based on only a 6-month reporting delay. Data for the year 2016 should not be used when assessing trends. Numbers less than 12, and trends based on these numbers, should be interpreted with caution. Data have been statistically adjusted to account for missing transmission category; therefore, values may not sum to column total. Data include persons with HIV infection attributed to heterosexual contact with a person known to have, or to be at high risk for, HIV infection.

a Data are based on residence at time of diagnosis of HIV infection.

$\mathrm{b}_{\text {Hispanics/Latinos can be of any race. }}$ 
Table 8a. Stage 3 (AIDS) attributed to male-to-male sexual contact and male-to-male sexual contact and injection drug use, by selected characteristics, 2011-2016-United States

\begin{tabular}{|c|c|c|c|c|c|c|c|c|c|c|c|}
\hline \multicolumn{6}{|c|}{ Male-to-male sexual contact } & \multicolumn{6}{|c|}{ Male-to-male sexual contact and injection drug use } \\
\hline 2011 & 2012 & 2013 & 2014 & 2015 & 2016 & 2011 & 2012 & 2013 & 2014 & 2015 & 2016 \\
\hline No. & No. & No. & No. & No. & No. & No. & No. & No. & No. & No. & No. \\
\hline
\end{tabular}

\section{American Indian/Alaska Native}

\section{Age at diagnosis (yr)}

$13-14$
$15-19$
$20-24$
$25-29$
$30-34$
$35-39$
$40-44$
$45-49$
$50-54$
$55-59$
$60-64$
$\geq 65$

0
0
3
4
8
9
9
6
2
1
2
0

$\begin{array}{rr}0 & 0 \\ 1 & 0 \\ 3 & 3 \\ 6 & 5 \\ 14 & 9 \\ 2 & 3 \\ 9 & 10 \\ 3 & 3 \\ 6 & 7 \\ 1 & 0 \\ 0 & 0 \\ 1 & 1\end{array}$

0
0
3
5
8
5
9
6
6
2
0
1

0
1
6
5
6
5
4
3
4
2
0
1

0
0
3
12
11
9
8
6
3
3
1
2

0
0
1
1
1
0
3
2
2
1
0
0

0
0
2
2
2
1
1
1
0
0
0
0

Region of residence ${ }^{a}$

Northeast

Midwest

South

West

Total

2
3
10
30
45

$\begin{array}{rr}3 & 1 \\ 2 & 3 \\ 14 & 9 \\ 26 & 29 \\ 45 & 42\end{array}$

1
4
9
30
44

$\begin{array}{rr}0 & 3 \\ 3 & 8 \\ 15 & 16 \\ 18 & 30 \\ 36 & 57\end{array}$

\begin{tabular}{rrrrrrr}
3 & 0 & 0 & 1 & 0 & 0 & 0 \\
8 & 2 & 1 & 0 & 2 & 0 & 1 \\
16 & 0 & 1 & 0 & 1 & 2 & 2 \\
30 & 10 & 9 & 5 & 3 & 4 & 4 \\
57 & 12 & 11 & 6 & 6 & 6 & 7 \\
\hline
\end{tabular}

Asian $^{b}$

Age at diagnosis (yr)

$13-14$
$15-19$
$20-24$
$25-29$
$30-34$
$35-39$
$40-44$
$45-49$
$50-54$
$55-59$
$60-64$
$\geq 65$

0
1
11
33
35
46
42
26
12
9
6
3

Region of residence ${ }^{a}$ Northeas

Midwest

South

West

33
22
52

$\begin{array}{rrr}0 & 0 & \\ 2 & 1 & \\ 9 & 21 & 16 \\ 30 & 34 & 26 \\ 31 & 39 & 32 \\ 45 & 32 & 39 \\ 41 & 51 & 37 \\ 39 & 35 & 39 \\ 20 & 20 & 13 \\ 11 & 8 & \\ 6 & 4 & 3 \\ 4 & 4 & 5\end{array}$

$\begin{array}{rrr}0 & 0 & 0 \\ 0 & 2 & 2 \\ 16 & 14 & 18 \\ 26 & 36 & 46 \\ 32 & 32 & 38 \\ 39 & 25 & 24 \\ 37 & 41 & 37 \\ 39 & 28 & 29 \\ 13 & 30 & 19 \\ 4 & 12 & 11 \\ 3 & 1 & 3 \\ 5 & 3 & 6\end{array}$

0
2
18
46
38
24
37
29
19
11
3
6

$\begin{array}{ll}0 & 0 \\ 0 & 0 \\ 0 & 1 \\ 1 & 2 \\ 1 & 0 \\ 4 & 2 \\ 1 & 4 \\ 2 & 0 \\ 0 & 1 \\ 0 & 0 \\ 0 & 1 \\ 0 & 0\end{array}$

$\begin{array}{llll}0 & 0 & 0 & 0 \\ 0 & 0 & 1 & 0 \\ 1 & 0 & 2 & 3 \\ 0 & 0 & 1 & 2 \\ 2 & 1 & 0 & 0 \\ 0 & 0 & 0 & 1 \\ 0 & 0 & 0 & 1 \\ 0 & 2 & 1 & 0 \\ 1 & 2 & 0 & 0 \\ 2 & 0 & 0 & 0 \\ 0 & 0 & 0 & 0 \\ 0 & 0 & 0 & 0\end{array}$

Total

$114 \quad 138$

$\begin{array}{rrrrr}35 & 35 & 40 & 46 & 38 \\ 16 & 23 & 25 & 29 & 23 \\ 49 & 51 & 48 & 32 & 56 \\ 138 & 139 & 101 & 118 & 115 \\ 238 & \mathbf{2 4 8} & \mathbf{2 1 4} & \mathbf{2 2 5} & \mathbf{2 3 2}\end{array}$

38
23
56
115
232

$\begin{array}{lr}0 & 1 \\ 0 & 1 \\ 1 & 2 \\ 8 & 6 \\ 9 & 10\end{array}$

2

6


Table 8a. Stage 3 (AIDS) attributed to male-to-male sexual contact and male-to-male sexual contact and injection drug use, by selected characteristics, 2011-2016-United States (cont)

\begin{tabular}{|c|c|c|c|c|c|c|c|c|c|c|c|c|}
\hline & \multicolumn{6}{|c|}{ Male-to-male sexual contact } & \multicolumn{6}{|c|}{ Male-to-male sexual contact and injection drug use } \\
\hline & 2011 & 2012 & 2013 & 2014 & 2015 & 2016 & 2011 & 2012 & 2013 & 2014 & 2015 & 2016 \\
\hline & No. & No. & No. & No. & No. & No. & No. & No. & No. & No. & No. & No. \\
\hline & \multicolumn{12}{|c|}{ Black/African American } \\
\hline \multicolumn{13}{|c|}{ Age at diagnosis (yr) } \\
\hline $13-14$ & 3 & 3 & 2 & 1 & 0 & 0 & 0 & 0 & 0 & 0 & 0 & 0 \\
\hline $15-19$ & 163 & 148 & 155 & 85 & 84 & 88 & 2 & 4 & 2 & 3 & 1 & 2 \\
\hline $20-24$ & 896 & 852 & 924 & 593 & 560 & 537 & 27 & 20 & 22 & 13 & 10 & 9 \\
\hline $25-29$ & 866 & 924 & 997 & 855 & 861 & 950 & 35 & 28 & 33 & 21 & 27 & 21 \\
\hline $30-34$ & 708 & 742 & 704 & 580 & 625 & 617 & 37 & 47 & 30 & 26 & 18 & 30 \\
\hline $35-39$ & 523 & 527 & 495 & 413 & 413 & 439 & 44 & 35 & 29 & 14 & 26 & 26 \\
\hline $40-44$ & 586 & 564 & 475 & 383 & 347 & 335 & 45 & 40 & 40 & 26 & 24 & 21 \\
\hline $45-49$ & 550 & 528 & 482 & 370 & 358 & 316 & 66 & 69 & 49 & 34 & 31 & 27 \\
\hline $50-54$ & 389 & 350 & 372 & 292 & 307 & 300 & 54 & 61 & 41 & 28 & 29 & 29 \\
\hline $55-59$ & 198 & 212 & 197 & 187 & 163 & 181 & 47 & 22 & 37 & 28 & 18 & 19 \\
\hline $60-64$ & 66 & 95 & 104 & 91 & 75 & 95 & 14 & 17 & 17 & 12 & 15 & 24 \\
\hline$\geq 65$ & 61 & 66 & 61 & 59 & 70 & 64 & 7 & 6 & 4 & 7 & 9 & 8 \\
\hline \multicolumn{13}{|c|}{ Region of residence ${ }^{a}$} \\
\hline Northeast & 683 & 637 & 655 & 486 & 471 & 466 & 58 & 59 & 39 & 24 & 28 & 24 \\
\hline Midwest & 788 & 784 & 798 & 624 & 582 & 642 & 54 & 54 & 50 & 36 & 31 & 32 \\
\hline South & 3,144 & 3,167 & 3,143 & 2,487 & 2,520 & 2,485 & 229 & 193 & 179 & 121 & 129 & 133 \\
\hline West & 394 & 423 & 372 & 310 & 290 & 329 & 39 & 42 & 35 & 31 & 19 & 27 \\
\hline Total & 5,009 & 5,011 & 4,968 & 3,907 & 3,863 & 3,922 & 380 & 348 & 303 & 212 & 207 & 216 \\
\hline
\end{tabular}

Age at diagnosis (yr)

\begin{tabular}{|c|c|c|c|c|c|c|c|c|c|c|c|c|}
\hline $13-14$ & 1 & 0 & 1 & 0 & 0 & 1 & 0 & 1 & 0 & 0 & 0 & 0 \\
\hline 15-19 & 46 & 42 & 51 & 17 & 15 & 19 & 3 & 2 & 3 & 0 & 1 & 1 \\
\hline $20-24$ & 298 & 350 & 293 & 234 & 213 & 219 & 26 & 20 & 13 & 9 & 9 & 12 \\
\hline $25-29$ & 536 & 519 & 516 & 428 & 414 & 460 & 37 & 22 & 29 & 24 & 32 & 16 \\
\hline 30-34 & 484 & 579 & 520 & 453 & 447 & 472 & 54 & 39 & 34 & 33 & 28 & 26 \\
\hline $35-39$ & 517 & 432 & 492 & 432 & 407 & 428 & 44 & 43 & 42 & 20 & 26 & 30 \\
\hline 40-44 & 488 & 428 & 424 & 334 & 317 & 292 & 41 & 43 & 29 & 21 & 22 & 23 \\
\hline $45-49$ & 398 & 390 & 391 & 320 & 295 & 278 & 37 & 44 & 29 & 17 & 26 & 19 \\
\hline $50-54$ & 234 & 216 & 239 & 183 & 206 & 226 & 22 & 28 & 25 & 14 & 17 & 20 \\
\hline $55-59$ & 114 & 120 & 112 & 104 & 111 & 111 & 15 & 8 & 9 & 12 & 12 & 4 \\
\hline $60-64$ & 55 & 60 & 51 & 54 & 50 & 55 & 4 & 5 & 5 & 7 & 5 & 4 \\
\hline$\geq 65$ & 51 & 39 & 46 & 41 & 34 & 50 & 2 & 1 & 3 & 3 & 2 & 1 \\
\hline \multicolumn{13}{|c|}{ Region of residence ${ }^{a}$} \\
\hline Northeast & 663 & 615 & 624 & 498 & 493 & 446 & 94 & 70 & 46 & 30 & 30 & 28 \\
\hline Midwest & 243 & 237 & 231 & 200 & 200 & 191 & 16 & 18 & 19 & 11 & 12 & 10 \\
\hline South & 1,243 & 1,192 & 1,217 & 1,017 & 1,016 & 1,115 & 71 & 73 & 79 & 58 & 63 & 58 \\
\hline West & 1,073 & 1,132 & 1,064 & 885 & 800 & 861 & 102 & 94 & 77 & 60 & 74 & 61 \\
\hline Total & 3,222 & 3,176 & 3,136 & 2,600 & 2,509 & 2,613 & 283 & 255 & 221 & 159 & 179 & 157 \\
\hline
\end{tabular}


Table 8a. Stage 3 (AIDS) attributed to male-to-male sexual contact and male-to-male sexual contact and injection drug use, by selected characteristics, 2011-2016-United States (cont)

\begin{tabular}{|c|c|c|c|c|c|c|c|c|c|c|c|}
\hline \multicolumn{6}{|c|}{ Male-to-male sexual contact } & \multicolumn{6}{|c|}{ Male-to-male sexual contact and injection drug use } \\
\hline 2011 & 2012 & 2013 & 2014 & 2015 & 2016 & 2011 & 2012 & 2013 & 2014 & 2015 & 2016 \\
\hline No. & No. & No. & No. & No. & No. & No. & No. & No. & No. & No. & No. \\
\hline
\end{tabular}

Age at diagnosis (yr)

$13-14$
$15-19$
$20-24$
$25-29$
$30-34$
$35-39$
$40-44$
$45-49$
$50-54$
$55-59$
$60-64$
$\geq 65$

$\begin{array}{lllllllllllll}0 & 0 & 0 & 0 & 0 & 0 & 0 & 0 & 0 & 0 & 0 & 0 \\ 0 & 0 & 0 & 0 & 0 & 0 & 0 & 0 & 0 & 0 & 0 & 0 \\ 4 & 2 & 0 & 0 & 3 & 1 & 1 & 0 & 0 & 0 & 1 & 0 \\ 4 & 3 & 5 & 0 & 3 & 1 & 0 & 0 & 0 & 0 & 0 & 1 \\ 3 & 3 & 2 & 3 & 1 & 2 & 0 & 0 & 0 & 1 & 0 & 0 \\ 3 & 3 & 2 & 3 & 2 & 4 & 0 & 0 & 0 & 0 & 0 & 0 \\ 3 & 1 & 2 & 1 & 1 & 0 & 0 & 0 & 0 & 0 & 0 & 0 \\ 2 & 3 & 2 & 2 & 3 & 0 & 0 & 1 & 0 & 0 & 0 & 0 \\ 1 & 3 & 2 & 1 & 4 & 0 & 0 & 0 & 0 & 0 & 0 & 0 \\ 1 & 1 & 0 & 0 & 0 & 0 & 0 & 0 & 0 & 0 & 0 & 0 \\ 0 & 1 & 0 & 1 & 0 & 0 & 0 & 0 & 0 & 0 & 0 & 0 \\ 1 & 0 & 0 & 0 & 0 & 0 & 0 & 0 & 0 & 0 & 0 & 0\end{array}$

Region of residence ${ }^{a}$

\begin{tabular}{|c|c|c|c|c|c|c|c|c|c|c|c|c|}
\hline Northeast & 2 & 1 & 0 & 0 & 2 & 0 & 0 & 0 & 0 & 0 & 0 & 1 \\
\hline Midwest & 0 & 2 & 0 & 1 & 0 & 0 & 0 & 0 & 0 & 0 & 0 & 0 \\
\hline South & 1 & 2 & 0 & 2 & 3 & 0 & 0 & 0 & 0 & 0 & 0 & 0 \\
\hline West & 19 & 14 & 15 & 8 & 12 & 8 & 1 & 1 & 0 & 1 & 1 & 0 \\
\hline Total & 22 & 19 & 15 & 11 & 17 & 8 & 1 & 1 & 0 & 1 & 1 & 1 \\
\hline
\end{tabular}

\section{Age at diagnosis (yr)}

\begin{tabular}{|c|c|c|c|c|c|c|c|c|c|c|c|c|}
\hline & & & & & & & & & & & & \\
\hline $13-14$ & 1 & 0 & 0 & 0 & 0 & 0 & 0 & 0 & 0 & 0 & 0 & 0 \\
\hline $15-19$ & 29 & 22 & 18 & 6 & 12 & 10 & 1 & 1 & 0 & 1 & 2 & 1 \\
\hline 20-24 & 177 & 160 & 177 & 113 & 107 & 91 & 19 & 17 & 15 & 18 & 12 & 13 \\
\hline $25-29$ & 318 & 368 & 334 & 264 & 276 & 249 & 45 & 40 & 36 & 30 & 28 & 28 \\
\hline 30-34 & 449 & 462 & 401 & 337 & 328 & 317 & 66 & 61 & 53 & 37 & 46 & 44 \\
\hline $35-39$ & 473 & 418 & 425 & 330 & 318 & 288 & 70 & 63 & 49 & 36 & 51 & 45 \\
\hline 40-44 & 763 & 619 & 585 & 400 & 351 & 324 & 77 & 82 & 61 & 52 & 44 & 39 \\
\hline $45-49$ & 851 & 734 & 663 & 561 & 466 & 437 & 85 & 104 & 66 & 58 & 42 & 45 \\
\hline $50-54$ & 597 & 631 & 620 & 519 & 536 & 504 & 53 & 57 & 57 & 57 & 51 & 54 \\
\hline $55-59$ & 362 & 339 & 381 & 315 & 312 & 334 & 28 & 32 & 33 & 25 & 32 & 28 \\
\hline $60-64$ & 186 & 213 & 226 & 182 & 171 & 198 & 15 & 10 & 18 & 11 & 17 & 14 \\
\hline$\geq 65$ & 134 & 160 & 167 & 166 & 166 & 158 & 6 & 10 & 9 & 5 & 7 & 5 \\
\hline \multicolumn{13}{|c|}{ Region of residence $^{a}$} \\
\hline Northeast & 607 & 590 & 512 & 424 & 422 & 398 & 47 & 56 & 31 & 37 & 32 & 31 \\
\hline Midwest & 782 & 768 & 739 & 597 & 586 & 540 & 67 & 56 & 66 & 49 & 51 & 48 \\
\hline South & 1,789 & 1,706 & 1,736 & 1,381 & 1,281 & 1,254 & 144 & 180 & 157 & 121 & 124 & 111 \\
\hline West & 1,161 & 1,061 & 1,009 & 790 & 755 & 718 & 207 & 184 & 143 & 123 & 125 & 124 \\
\hline Total & 4,339 & 4,125 & 3,996 & 3,192 & 3,044 & 2,910 & 465 & 476 & 397 & 330 & 332 & 314 \\
\hline
\end{tabular}


Table 8a. Stage 3 (AIDS) attributed to male-to-male sexual contact and male-to-male sexual contact and injection drug use, by selected characteristics, 2011-2016-United States (cont)



Note. Data for the year 2016 are preliminary (subject to change) because they are based on only a 6-month reporting delay. Data for the year 2016 should not be used when assessing trends. Numbers less than 12, and trends based on these numbers, should be interpreted with caution. Data have been statistically adjusted to account for missing transmission category; therefore, values may not sum to column total.

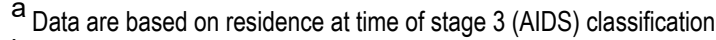



${ }^{\mathrm{C}}$ Hispanics/Latinos can be of any race. 
Table 8b. Stage 3 (AIDS) attributed to male-to-male sexual contact and male-to-male sexual contact and injection drug use, by selected characteristics, 2011-2016-United States and 6 dependent areas

\begin{tabular}{|c|c|c|c|c|c|c|c|c|c|c|c|}
\hline \multicolumn{6}{|c|}{ Male-to-male sexual contact } & \multicolumn{6}{|c|}{ Male-to-male sexual contact and injection drug use } \\
\hline 2011 & 2012 & 2013 & 2014 & 2015 & 2016 & 2011 & 2012 & 2013 & 2014 & 2015 & 2016 \\
\hline No. & No. & No. & No. & No. & No. & No. & No. & No. & No. & No. & No. \\
\hline
\end{tabular}

\section{American Indian/Alaska Native}

Age at diagnosis (yr)

\begin{tabular}{|c|c|c|c|c|c|c|c|c|c|c|c|}
\hline $13-14$ & 0 & 0 & 0 & 0 & 0 & 0 & 0 & 0 & 0 & 0 & 0 \\
\hline $15-19$ & 0 & 1 & 0 & 0 & 1 & 0 & 0 & 0 & 0 & 0 & 1 \\
\hline $20-24$ & 3 & 3 & 3 & 3 & 6 & 3 & 1 & 2 & 1 & 0 & 2 \\
\hline $25-29$ & 4 & 6 & 5 & 5 & 5 & 12 & 1 & 2 & 0 & 0 & 1 \\
\hline $30-34$ & 8 & 14 & 9 & 8 & 6 & 11 & 1 & 2 & 2 & 1 & 0 \\
\hline $35-39$ & 9 & 2 & 3 & 5 & 5 & 9 & 0 & 1 & 0 & 0 & 0 \\
\hline $40-44$ & 9 & 9 & 10 & 9 & 4 & 8 & 3 & 1 & 0 & 0 & 0 \\
\hline $45-49$ & 6 & 3 & 3 & 6 & 3 & 6 & 2 & 1 & 0 & 2 & 1 \\
\hline $50-54$ & 2 & 6 & 7 & 6 & 4 & 3 & 2 & 0 & 1 & 2 & 0 \\
\hline $55-59$ & 1 & 1 & 0 & 2 & 2 & 3 & 1 & 0 & 2 & 0 & 0 \\
\hline $60-64$ & 2 & 0 & 0 & 0 & 0 & 1 & 0 & 0 & 0 & 0 & 0 \\
\hline$\geq 65$ & 0 & 1 & 1 & 1 & 1 & 2 & 0 & 0 & 0 & 0 & 0 \\
\hline
\end{tabular}

Region of residence ${ }^{a}$

Northeast

Midwest

South

West

U.S. dependent areas

Total

Age at diagnosis $(\mathrm{yr})$

13-14

15-19

20-24

25-29

30-34

35-39

40-44

$45-49$

50-54

$55-59$

60-64

$\geq 65$

Region of residence ${ }^{a}$ Northeast

Midwest

South

West

U.S. dependent areas

Total

$\begin{array}{rr}2 & \\ 3 & \\ 10 & 14 \\ 30 & 26 \\ 0 & \end{array}$

$\begin{array}{rr}3 & 1 \\ 2 & 3 \\ 14 & 9 \\ 26 & 29 \\ 0 & 0 \\ 45 & 42\end{array}$

\begin{tabular}{rrrrrrrrr}
1 & 0 & 3 & 0 & 0 & 1 & 0 & 0 & 0 \\
4 & 3 & 8 & 2 & 1 & 0 & 2 & 0 & 1 \\
9 & 15 & 16 & 0 & 1 & 0 & 1 & 2 & 2 \\
30 & 18 & 30 & 10 & 9 & 5 & 3 & 4 & 4 \\
0 & 0 & 0 & 0 & 0 & 0 & 0 & 0 & 0 \\
44 & 36 & 57 & 12 & 11 & 6 & 6 & 6 & 7 \\
\hline
\end{tabular}

\section{Asian $^{\mathrm{b}}$}

$\begin{array}{rrrrrrrrrrrrr}0 & 0 & 0 & 0 & 0 & 0 & 0 & 0 & 0 & 0 & 0 & 0 \\ 1 & 2 & 1 & 0 & 2 & 2 & 0 & 0 & 0 & 0 & 0 & 0 \\ 11 & 9 & 21 & 16 & 14 & 18 & 0 & 1 & 0 & 0 & 0 & 0 \\ 33 & 30 & 34 & 26 & 37 & 46 & 1 & 2 & 1 & 3 & 0 & 0 \\ 35 & 31 & 39 & 32 & 32 & 38 & 1 & 0 & 1 & 0 & 1 & 1 \\ 47 & 45 & 32 & 39 & 25 & 24 & 4 & 2 & 1 & 2 & 0 & 0 \\ 42 & 41 & 51 & 37 & 41 & 37 & 1 & 4 & 4 & 6 & 2 & 0 \\ 26 & 39 & 35 & 39 & 28 & 29 & 2 & 0 & 0 & 0 & 1 & 0 \\ 12 & 20 & 20 & 14 & 30 & 19 & 0 & 1 & 0 & 0 & 1 & 1 \\ 9 & 11 & 8 & 4 & 12 & 11 & 0 & 0 & 0 & 0 & 0 & 0 \\ 6 & 6 & 4 & 3 & 1 & 3 & 0 & 1 & 0 & 0 & 0 & 0 \\ 3 & 4 & 4 & 5 & 3 & 6 & 0 & 0 & 0 & 0 & 0 & 0\end{array}$


Table 8b. Stage 3 (AIDS) attributed to male-to-male sexual contact and male-to-male sexual contact and injection drug use, by selected characteristics, 2011-2016-United States and 6 dependent areas (cont)

\begin{tabular}{|c|c|c|c|c|c|c|c|c|c|c|c|c|}
\hline & \multicolumn{6}{|c|}{ Male-to-male sexual contact } & \multicolumn{6}{|c|}{ Male-to-male sexual contact and injection drug use } \\
\hline & 2011 & 2012 & 2013 & 2014 & 2015 & 2016 & 2011 & 2012 & 2013 & 2014 & 2015 & 2016 \\
\hline & No. & No. & No. & No. & No. & No. & No. & No. & No. & No. & No. & No. \\
\hline & \multicolumn{12}{|c|}{ Black/African American } \\
\hline \multicolumn{13}{|l|}{ Age at diagnosis (yr) } \\
\hline $13-14$ & 3 & 3 & 2 & 1 & 0 & 0 & 0 & 0 & 0 & 0 & 0 & 0 \\
\hline $15-19$ & 163 & 148 & 155 & 85 & 84 & 88 & 2 & 4 & 2 & 3 & 1 & 2 \\
\hline $20-24$ & 896 & 852 & 924 & 593 & 561 & 537 & 27 & 20 & 22 & 13 & 10 & 9 \\
\hline $25-29$ & 866 & 925 & 997 & 855 & 861 & 950 & 35 & 28 & 33 & 21 & 27 & 21 \\
\hline 30-34 & 708 & 742 & 704 & 580 & 625 & 617 & 37 & 47 & 30 & 26 & 18 & 30 \\
\hline $35-39$ & 523 & 528 & 495 & 413 & 414 & 439 & 44 & 35 & 29 & 14 & 26 & 26 \\
\hline $40-44$ & 586 & 565 & 475 & 384 & 347 & 335 & 45 & 40 & 40 & 26 & 24 & 21 \\
\hline $45-49$ & 550 & 528 & 482 & 371 & 359 & 316 & 66 & 69 & 49 & 34 & 31 & 27 \\
\hline $50-54$ & 390 & 350 & 372 & 292 & 307 & 300 & 54 & 61 & 41 & 28 & 29 & 29 \\
\hline $55-59$ & 199 & 212 & 197 & 187 & 163 & 181 & 47 & 22 & 37 & 28 & 18 & 19 \\
\hline $60-64$ & 66 & 95 & 104 & 91 & 75 & 95 & 14 & 17 & 17 & 12 & 15 & 24 \\
\hline$\geq 65$ & 61 & 66 & 61 & 59 & 70 & 64 & 7 & 6 & 4 & 7 & 9 & 8 \\
\hline \multicolumn{13}{|l|}{ Region of residence ${ }^{a}$} \\
\hline Northeast & 683 & 637 & 655 & 486 & 471 & 466 & 58 & 59 & 39 & 24 & 28 & 24 \\
\hline Midwest & 788 & 784 & 798 & 624 & 582 & 642 & 54 & 54 & 50 & 36 & 31 & 32 \\
\hline South & 3,144 & 3,167 & 3,143 & 2,487 & 2,520 & 2,485 & 229 & 193 & 179 & 121 & 129 & 133 \\
\hline West & 394 & 423 & 372 & 310 & 290 & 329 & 39 & 42 & 35 & 31 & 19 & 27 \\
\hline U.S. dependent areas & 1 & 3 & 0 & 1 & 3 & 0 & 0 & 0 & 0 & 0 & 0 & 0 \\
\hline Total & 5,010 & 5,014 & 4,968 & 3,908 & 3,866 & 3,922 & 380 & 348 & 303 & 212 & 207 & 216 \\
\hline
\end{tabular}

\section{Age at diagnosis (yr)}

\begin{tabular}{|c|c|c|c|c|c|c|c|c|c|c|c|c|}
\hline $13-14$ & 2 & 0 & 1 & 0 & 0 & 1 & 0 & 1 & 0 & 0 & 0 & 0 \\
\hline $15-19$ & 47 & 42 & 52 & 19 & 15 & 22 & 4 & 2 & 3 & 0 & 1 & 1 \\
\hline $20-24$ & 307 & 361 & 309 & 249 & 221 & 227 & 26 & 20 & 14 & 9 & 9 & 12 \\
\hline $25-29$ & 549 & 539 & 534 & 438 & 429 & 467 & 39 & 24 & 29 & 24 & 32 & 16 \\
\hline $30-34$ & 496 & 589 & 544 & 467 & 457 & 483 & 57 & 40 & 36 & 33 & 29 & 27 \\
\hline 35-39 & 537 & 443 & 507 & 443 & 418 & 446 & 48 & 50 & 45 & 22 & 26 & 32 \\
\hline 40-44 & 503 & 447 & 445 & 346 & 329 & 299 & 45 & 46 & 32 & 25 & 24 & 30 \\
\hline $45-49$ & 420 & 410 & 404 & 333 & 304 & 288 & 40 & 47 & 30 & 19 & 26 & 21 \\
\hline 50-54 & 245 & 228 & 256 & 190 & 214 & 235 & 26 & 29 & 27 & 16 & 23 & 22 \\
\hline $55-59$ & 122 & 126 & 115 & 107 & 117 & 115 & 16 & 10 & 9 & 12 & 15 & 7 \\
\hline $60-64$ & 57 & 62 & 58 & 61 & 52 & 57 & 5 & 5 & 5 & 7 & 5 & 5 \\
\hline$\geq 65$ & 55 & 43 & 50 & 44 & 36 & 51 & 2 & 3 & 3 & 3 & 3 & 1 \\
\hline \multicolumn{13}{|l|}{ Region of residence ${ }^{a}$} \\
\hline Northeast & 663 & 615 & 624 & 498 & 493 & 446 & 94 & 70 & 46 & 30 & 30 & 28 \\
\hline Midwest & 243 & 237 & 231 & 200 & 200 & 191 & 16 & 18 & 19 & 11 & 12 & 10 \\
\hline South & 1,243 & 1,192 & 1,217 & 1,017 & 1,016 & 1,115 & 71 & 73 & 79 & 58 & 63 & 58 \\
\hline West & 1,073 & 1,132 & 1,064 & 885 & 800 & 861 & 102 & 94 & 77 & 60 & 74 & 61 \\
\hline U.S. dependent areas & 117 & 114 & 137 & 96 & 82 & 79 & 23 & 21 & 12 & 9 & 13 & 18 \\
\hline Total & 3,339 & 3,290 & 3,273 & 2,696 & 2,591 & 2,692 & 306 & 276 & 233 & 168 & 192 & 175 \\
\hline
\end{tabular}


Table 8b. Stage 3 (AIDS) attributed to male-to-male sexual contact and male-to-male sexual contact and injection drug use, by selected characteristics, 2011-2016-United States and 6 dependent areas (cont)

\begin{tabular}{|c|c|c|c|c|c|c|c|c|c|c|c|}
\hline \multicolumn{6}{|c|}{ Male-to-male sexual contact } & \multicolumn{6}{|c|}{ Male-to-male sexual contact and injection drug use } \\
\hline 2011 & 2012 & 2013 & 2014 & 2015 & 2016 & 2011 & 2012 & 2013 & 2014 & 2015 & 2016 \\
\hline No. & No. & No. & No. & No. & No. & No. & No. & No. & No. & No. & No. \\
\hline
\end{tabular}

Age at diagnosis (yr)

13-14

15-19

20-24

25-29

30-34

$35-39$

40-44

45-49

50-54

$55-59$

$60-64$

$\geq 65$

Region of residence ${ }^{a}$

Northeast

Midwest

South

West

U.S. dependent areas

Total

$\begin{array}{lll}0 & 0 & 0 \\ 0 & 0 & 0 \\ 4 & 2 & 0 \\ 4 & 3 & 5 \\ 3 & 4 & 2 \\ 3 & 3 & 2 \\ 3 & 1 & 2 \\ 2 & 3 & 2 \\ 1 & 3 & 2 \\ 1 & 1 & 0 \\ 0 & 1 & 0 \\ 1 & 0 & 0\end{array}$

\section{Native Hawaiian/Other Pacific Islander}

0
0
0
0
3
3
1
2
1
0
1
0

$\begin{array}{llll}0 & 0 & 0 & 0 \\ 0 & 0 & 0 & 0 \\ 3 & 2 & 1 & 0 \\ 4 & 1 & 0 & 0 \\ 1 & 4 & 0 & 0 \\ 2 & 4 & 0 & 0 \\ 1 & 0 & 0 & 0 \\ 3 & 0 & 0 & 1 \\ 4 & 0 & 0 & 0 \\ 0 & 1 & 0 & 0 \\ 0 & 0 & 0 & 0 \\ 0 & 0 & 0 & 0\end{array}$

$\begin{array}{llll}0 & 0 & 0 & 0 \\ 0 & 0 & 0 & 0 \\ 0 & 0 & 1 & 0 \\ 0 & 0 & 0 & 1 \\ 0 & 1 & 0 & 0 \\ 0 & 0 & 0 & 0 \\ 0 & 0 & 0 & 0 \\ 0 & 0 & 0 & 0 \\ 0 & 0 & 0 & 0 \\ 0 & 0 & 0 & 0 \\ 0 & 0 & 0 & 0 \\ 0 & 0 & 0 & 0\end{array}$

Age at diagnosis (yr)

$13-14$
$15-19$
$20-24$
$25-29$
$30-34$
$35-39$
$40-44$
$45-49$
$50-54$
$55-59$
$60-64$
$\geq 65$

$\begin{array}{rrrrrr}1 & 0 & 0 & 0 & 0 & 0 \\ 29 & 22 & 18 & 6 & 12 & 10 \\ 177 & 160 & 177 & 113 & 107 & 91 \\ 318 & 368 & 334 & 264 & 276 & 249 \\ 449 & 462 & 401 & 337 & 328 & 317 \\ 473 & 418 & 425 & 330 & 318 & 288 \\ 763 & 620 & 585 & 400 & 351 & 324 \\ 851 & 734 & 663 & 561 & 466 & 437 \\ 597 & 631 & 620 & 519 & 537 & 504 \\ 362 & 339 & 381 & 316 & 312 & 334 \\ 186 & 214 & 226 & 182 & 171 & 198 \\ 134 & 160 & 167 & 166 & 166 & 158\end{array}$

Region of residence ${ }^{a}$ Northeast

Midwest

South

West

U.S. dependent areas

Total

$\begin{array}{rr}2 & 1 \\ 0 & 2 \\ 1 & 2 \\ 19 & 14 \\ 0 & 1 \\ 22 & 20\end{array}$

$\begin{array}{rr}0 & 0 \\ 0 & 1 \\ 0 & 2 \\ 15 & 8 \\ 0 & 0 \\ 15 & 11\end{array}$

2
0
3
12
2
19

\begin{tabular}{rrrrrrr}
0 & 0 & 0 & 0 & 0 & 0 & 1 \\
0 & 0 & 0 & 0 & 0 & 0 & 0 \\
0 & 0 & 0 & 0 & 0 & 0 & 0 \\
8 & 1 & 1 & 0 & 1 & 1 & 0 \\
4 & 0 & 0 & 0 & 0 & 0 & 0 \\
12 & 1 & 1 & 0 & 1 & 1 & 1 \\
\hline
\end{tabular}

\section{White}

\begin{tabular}{|c|c|c|c|c|c|c|c|c|c|c|c|}
\hline 607 & 590 & 512 & 424 & 422 & 398 & 47 & 56 & 31 & 37 & 32 & 31 \\
\hline 782 & 768 & 739 & 597 & 586 & 540 & 67 & 56 & 66 & 49 & 51 & 48 \\
\hline 1,789 & 1,706 & 1,736 & 1,381 & 1,281 & 1,254 & 144 & 180 & 157 & 121 & 124 & 111 \\
\hline 1,161 & 1,061 & 1,009 & 790 & 755 & 718 & 207 & 184 & 143 & 123 & 125 & 124 \\
\hline 0 & 2 & 0 & 1 & 1 & 0 & 0 & 0 & 0 & 0 & 0 & 0 \\
\hline 4,339 & 4,127 & 3,996 & 3,193 & 3,045 & 2,910 & 465 & 476 & 397 & 330 & 332 & 314 \\
\hline
\end{tabular}


Table 8b. Stage 3 (AIDS) attributed to male-to-male sexual contact and male-to-male sexual contact and injection drug use, by selected characteristics, 2011-2016-United States and 6 dependent areas (cont)



Total

Age at diagnosis (yr)

\begin{tabular}{|c|c|c|c|c|c|c|c|c|c|c|c|c|}
\hline $13-14$ & 7 & 3 & 2 & 1 & 0 & 1 & 0 & 1 & 0 & 0 & 0 & 0 \\
\hline $15-19$ & 255 & 218 & 249 & 114 & 116 & 126 & 8 & 8 & 6 & 4 & 6 & 6 \\
\hline $20-24$ & 1,473 & 1,463 & 1,537 & 1,023 & 955 & 904 & 84 & 67 & 55 & 43 & 38 & 40 \\
\hline $25-29$ & 1,866 & 1,968 & 1,993 & 1,668 & 1,689 & 1,787 & 131 & 100 & 104 & 87 & 95 & 72 \\
\hline 30-34 & 1,782 & 1,937 & 1,774 & 1,479 & 1,490 & 1,525 & 177 & 161 & 130 & 107 & 102 & 111 \\
\hline 35-39 & 1,656 & 1,508 & 1,544 & 1,281 & 1,233 & 1,251 & 178 & 161 & 134 & 81 & 106 & 110 \\
\hline 40-44 & 2,003 & 1,761 & 1,626 & 1,233 & 1,110 & 1,028 & 188 & 184 & 147 & 115 & 100 & 97 \\
\hline $45-49$ & 1,937 & 1,789 & 1,658 & 1,367 & 1,204 & 1,113 & 212 & 242 & 159 & 122 & 108 & 96 \\
\hline 50-54 & 1,306 & 1,287 & 1,317 & 1,068 & 1,132 & 1,103 & 147 & 157 & 135 & 111 & 113 & 113 \\
\hline $55-59$ & 725 & 727 & 732 & 645 & 629 & 669 & 103 & 68 & 86 & 73 & 70 & 58 \\
\hline 60-64 & 335 & 390 & 407 & 348 & 312 & 362 & 34 & 35 & 43 & 33 & 41 & 49 \\
\hline$\geq 65$ & 267 & 280 & 293 & 287 & 285 & 288 & 16 & 20 & 19 & 15 & 20 & 15 \\
\hline \multicolumn{13}{|l|}{ Region of residence ${ }^{\mathrm{a}}$} \\
\hline Northeast & 2,095 & 2,028 & 1,946 & 1,528 & 1,526 & 1,435 & 224 & 204 & 130 & 102 & 99 & 95 \\
\hline Midwest & 1,953 & 1,881 & 1,877 & 1,522 & 1,453 & 1,443 & 151 & 139 & 144 & 110 & 105 & 101 \\
\hline South & 6,546 & 6,418 & 6,438 & 5,161 & 5,031 & 5,070 & 489 & 485 & 449 & 326 & 337 & 326 \\
\hline West & 2,899 & 2,886 & 2,737 & 2,203 & 2,057 & 2,127 & 391 & 357 & 282 & 244 & 245 & 229 \\
\hline U.S. dependent areas & 119 & 120 & 138 & 100 & 89 & 83 & 23 & 21 & 12 & 9 & 13 & 18 \\
\hline Total & 13,612 & 13,332 & 13,134 & 10,513 & 10,155 & 10,157 & 1,279 & 1,206 & 1,018 & 791 & 800 & 769 \\
\hline
\end{tabular}

Note. Data for the year 2016 are preliminary (subject to change) because they are based on only a 6-month reporting delay. Data for the year 2016 should not be used when assessing trends. Numbers less than 12 , and trends based on these numbers, should be interpreted with caution. Data have been statistically adjusted to account for missing transmission category; therefore, values may not sum to column total.

$\mathrm{a}$ Data are based on residence at time of stage 3 (AIDS) classification.



${ }^{\mathrm{C}}$ Hispanics/Latinos can be of any race. 
Table 9a. Stage 3 (AIDS) attributed to injection drug use, by selected characteristics, 2011-2016-United States

\begin{tabular}{|c|c|c|c|c|c|c|c|c|c|c|c|}
\hline \multicolumn{6}{|c|}{ Male } & \multicolumn{6}{|c|}{ Female } \\
\hline 2011 & 2012 & 2013 & 2014 & 2015 & 2016 & 2011 & 2012 & 2013 & 2014 & 2015 & 2016 \\
\hline No. & No. & No. & No. & No. & No. & No. & No. & No. & No. & No. & No. \\
\hline
\end{tabular}

American Indian/Alaska Native

Age at diagnosis (yr)

$13-14$
$15-19$
$20-24$
$25-29$
$30-34$
$35-39$
$40-44$
$45-49$
$50-54$
$55-59$
$60-64$
$\geq 65$

$\begin{array}{llll}0 & 0 & 0 & 0 \\ 0 & 0 & 0 & 0 \\ 0 & 0 & 0 & 0 \\ 0 & 2 & 0 & 2 \\ 1 & 1 & 1 & 1 \\ 2 & 0 & 0 & 0 \\ 0 & 0 & 0 & 4 \\ 3 & 1 & 2 & 1 \\ 1 & 1 & 1 & 2 \\ 2 & 1 & 0 & 1 \\ 0 & 0 & 0 & 0 \\ 0 & 0 & 1 & 0\end{array}$

$\begin{array}{ll}0 & 0 \\ 0 & 0 \\ 0 & 0 \\ 0 & 0 \\ 2 & 0 \\ 0 & 0 \\ 1 & 1 \\ 0 & 3 \\ 2 & 0 \\ 1 & 1 \\ 0 & 0 \\ 0 & 0\end{array}$

0
0
0
0
0
0
1
3
0
1
0
0

Region of residence ${ }^{a}$ Northeast Midwest

South

West

$\begin{array}{rrrr}0 & 0 & 0 & 0 \\ 0 & 2 & 0 & 1 \\ 3 & 1 & 2 & 4 \\ 6 & 4 & 3 & 6 \\ 9 & 7 & 5 & 11\end{array}$

Total

(a)

Age at diagnosis $(\mathbf{y r})$
$13-14$
$15-19$
$20-24$
$25-29$
$30-34$
$35-39$
$40-44$
$45-49$
$50-54$
$55-59$
$60-64$
$\geq 65$

\section{Region of residence ${ }^{a}$}

Northeast

Midwest

South

West

Total

$\begin{array}{lll}0 & 0 & 0 \\ 0 & 0 & 0 \\ 0 & 0 & 0 \\ 1 & 0 & 0 \\ 3 & 2 & 2 \\ 1 & 5 & 1 \\ 4 & 4 & 1 \\ 2 & 2 & 1 \\ 1 & 1 & 0 \\ 0 & 1 & 2 \\ 0 & 0 & 1 \\ 0 & 0 & 0\end{array}$

$\begin{array}{ll}0 & 0 \\ 0 & 0 \\ 0 & 1 \\ 0 & 0 \\ 1 & 4 \\ 2 & 3 \\ 2 & 1 \\ 0 & 1 \\ 1 & 1 \\ 0 & 1 \\ 0 & 1 \\ 1 & 1\end{array}$

\begin{tabular}{rrrrrrrr}
0 & 1 & 0 & 1 & 0 & 0 & 0 & 0 \\
0 & 0 & 4 & 3 & 0 & 0 & 2 & 1 \\
0 & 1 & 1 & 3 & 2 & 3 & 5 & 1 \\
5 & 5 & 6 & 3 & 4 & 5 & 4 & 6 \\
5 & 7 & 11 & 10 & 6 & 8 & 11 & 8 \\
\hline
\end{tabular}

\section{Asian $^{b}$}

$\begin{array}{rrrrrrrrrrrrr}4 & 3 & 3 & 3 & 5 & 4 & 1 & 3 & 0 & 0 & 1 & 1 \\ 2 & 0 & 0 & 0 & 1 & 2 & 0 & 0 & 0 & 0 & 0 & 1 \\ 3 & 5 & 3 & 5 & 2 & 2 & 0 & 0 & 1 & 0 & 1 & 1 \\ 5 & 7 & 3 & 1 & 8 & 4 & 4 & 1 & 2 & 2 & 1 & 1 \\ 14 & 15 & 9 & 9 & 16 & 12 & 5 & 4 & 3 & 2 & 3 & 4\end{array}$


Table 9a. Stage 3 (AIDS) attributed to injection drug use, by selected characteristics, 2011-2016-United States (cont)

\begin{tabular}{|c|c|c|c|c|c|c|c|c|c|c|c|c|}
\hline & \multicolumn{6}{|c|}{ Male } & \multicolumn{6}{|c|}{ Female } \\
\hline & 2011 & 2012 & 2013 & 2014 & 2015 & 2016 & 2011 & 2012 & 2013 & 2014 & 2015 & 2016 \\
\hline & No. & No. & No. & No. & No. & No. & No. & No. & No. & No. & No. & No. \\
\hline & \multicolumn{12}{|c|}{ Black/African American } \\
\hline \multicolumn{13}{|c|}{ Age at diagnosis (yr) } \\
\hline $13-14$ & 0 & 0 & 0 & 0 & 0 & 0 & 0 & 0 & 0 & 0 & 0 & 0 \\
\hline $15-19$ & 1 & 3 & 2 & 1 & 0 & 1 & 4 & 3 & 1 & 2 & 1 & 1 \\
\hline $20-24$ & 7 & 12 & 8 & 5 & 4 & 4 & 17 & 14 & 7 & 9 & 9 & 6 \\
\hline $25-29$ & 23 & 25 & 21 & 12 & 20 & 12 & 39 & 27 & 25 & 17 & 17 & 13 \\
\hline $30-34$ & 41 & 38 & 38 & 22 & 19 & 20 & 54 & 49 & 37 & 24 & 27 & 25 \\
\hline $35-39$ & 57 & 56 & 42 & 46 & 28 & 42 & 79 & 68 & 59 & 37 & 34 & 41 \\
\hline $40-44$ & 99 & 91 & 55 & 49 & 38 & 38 & 106 & 97 & 64 & 65 & 39 & 42 \\
\hline $45-49$ & 139 & 118 & 118 & 80 & 60 & 47 & 145 & 110 & 83 & 74 & 54 & 45 \\
\hline $50-54$ & 169 & 169 & 141 & 87 & 91 & 67 & 121 & 114 & 89 & 80 & 55 & 50 \\
\hline $55-59$ & 135 & 141 & 109 & 90 & 84 & 76 & 85 & 75 & 80 & 57 & 43 & 49 \\
\hline $60-64$ & 67 & 71 & 71 & 64 & 62 & 58 & 29 & 37 & 35 & 20 & 34 & 34 \\
\hline$\geq 65$ & 40 & 55 & 50 & 51 & 40 & 41 & 20 & 25 & 21 & 20 & 15 & 20 \\
\hline \multicolumn{13}{|c|}{ Region of residence ${ }^{a}$} \\
\hline Northeast & 238 & 217 & 197 & 139 & 124 & 117 & 190 & 162 & 112 & 113 & 93 & 70 \\
\hline Midwest & 74 & 82 & 68 & 39 & 51 & 41 & 81 & 79 & 69 & 60 & 29 & 46 \\
\hline South & 423 & 440 & 351 & 302 & 251 & 220 & 383 & 338 & 293 & 212 & 179 & 187 \\
\hline West & 42 & 38 & 39 & 26 & 21 & 27 & 46 & 38 & 27 & 21 & 27 & 23 \\
\hline \multirow[t]{2}{*}{ Total } & 777 & 777 & 655 & 506 & 447 & 405 & 700 & 617 & 501 & 406 & 328 & 326 \\
\hline & \multicolumn{12}{|c|}{ Hispanic/Latino ${ }^{C}$} \\
\hline \multicolumn{13}{|c|}{ Age at diagnosis (yr) } \\
\hline $13-14$ & 0 & 0 & 0 & 0 & 0 & 0 & 0 & 0 & 0 & 0 & 0 & 0 \\
\hline $15-19$ & 0 & 0 & 0 & 0 & 0 & 0 & 0 & 0 & 2 & 0 & 0 & 0 \\
\hline $20-24$ & 10 & 4 & 8 & 2 & 6 & 5 & 3 & 4 & 5 & 5 & 6 & 3 \\
\hline $25-29$ & 33 & 19 & 13 & 16 & 13 & 17 & 14 & 12 & 10 & 13 & 8 & 3 \\
\hline $30-34$ & 38 & 43 & 34 & 25 & 29 & 25 & 26 & 17 & 20 & 14 & 13 & 12 \\
\hline $35-39$ & 46 & 45 & 36 & 44 & 36 & 31 & 22 & 24 & 18 & 19 & 17 & 23 \\
\hline $40-44$ & 75 & 48 & 47 & 41 & 37 & 30 & 35 & 30 & 28 & 19 & 18 & 13 \\
\hline $45-49$ & 79 & 80 & 70 & 45 & 38 & 32 & 35 & 35 & 29 & 24 & 25 & 20 \\
\hline $50-54$ & 67 & 70 & 49 & 51 & 39 & 31 & 38 & 26 & 24 & 25 & 18 & 19 \\
\hline $55-59$ & 49 & 40 & 37 & 43 & 29 & 33 & 21 & 17 & 15 & 17 & 9 & 9 \\
\hline $60-64$ & 21 & 30 & 25 & 15 & 16 & 20 & 8 & 12 & 12 & 6 & 8 & 5 \\
\hline$\geq 65$ & 17 & 16 & 13 & 14 & 15 & 25 & 5 & 3 & 9 & 3 & 8 & 6 \\
\hline \multicolumn{13}{|c|}{ Region of residence $^{a}$} \\
\hline Northeast & 225 & 193 & 170 & 132 & 111 & 108 & 113 & 90 & 96 & 71 & 71 & 51 \\
\hline Midwest & 30 & 20 & 16 & 13 & 18 & 10 & 10 & 12 & 6 & 7 & 6 & 7 \\
\hline South & 104 & 115 & 82 & 84 & 71 & 78 & 58 & 47 & 47 & 39 & 40 & 32 \\
\hline West & 75 & 66 & 64 & 67 & 60 & 52 & 27 & 31 & 23 & 27 & 15 & 23 \\
\hline Total & 434 & 394 & 332 & 296 & 260 & 248 & 208 & 180 & 172 & 144 & 132 & 113 \\
\hline
\end{tabular}


Table 9a. Stage 3 (AIDS) attributed to injection drug use, by selected characteristics, 2011-2016-United States (cont)

\begin{tabular}{|c|c|c|c|c|c|c|c|c|c|c|c|c|}
\hline & \multicolumn{6}{|c|}{ Male } & \multicolumn{6}{|c|}{ Female } \\
\hline & 2011 & 2012 & 2013 & 2014 & 2015 & 2016 & 2011 & 2012 & 2013 & 2014 & 2015 & 2016 \\
\hline & No. & No. & No. & No. & No. & No. & No. & No. & No. & No. & No. & No. \\
\hline & \multicolumn{12}{|c|}{ Native Hawaiian/Other Pacific Islander } \\
\hline \multicolumn{13}{|c|}{ Age at diagnosis (yr) } \\
\hline $13-14$ & 0 & 0 & 0 & 0 & 0 & 0 & 0 & 0 & 0 & 0 & 0 & 0 \\
\hline $15-19$ & 0 & 0 & 0 & 0 & 0 & 0 & 0 & 0 & 0 & 0 & 0 & 0 \\
\hline $20-24$ & 0 & 0 & 0 & 0 & 0 & 0 & 0 & 0 & 0 & 0 & 0 & 0 \\
\hline $25-29$ & 0 & 0 & 0 & 0 & 0 & 0 & 0 & 0 & 0 & 0 & 0 & 0 \\
\hline $30-34$ & 0 & 0 & 0 & 0 & 0 & 0 & 0 & 0 & 0 & 0 & 0 & 0 \\
\hline $35-39$ & 1 & 0 & 0 & 0 & 0 & 1 & 1 & 0 & 0 & 0 & 0 & 0 \\
\hline $40-44$ & 0 & 0 & 0 & 0 & 0 & 0 & 0 & 0 & 0 & 0 & 0 & 0 \\
\hline $45-49$ & 0 & 0 & 1 & 0 & 0 & 0 & 0 & 0 & 0 & 0 & 0 & 0 \\
\hline $50-54$ & 0 & 0 & 0 & 0 & 0 & 0 & 0 & 0 & 0 & 0 & 0 & 0 \\
\hline $55-59$ & 0 & 0 & 0 & 0 & 0 & 0 & 0 & 0 & 0 & 0 & 1 & 0 \\
\hline $60-64$ & 0 & 0 & 0 & 0 & 0 & 0 & 0 & 0 & 0 & 0 & 0 & 0 \\
\hline$\geq 65$ & 0 & 0 & 0 & 0 & 0 & 0 & 0 & 0 & 0 & 0 & 0 & 0 \\
\hline \multicolumn{13}{|c|}{ Region of residence ${ }^{a}$} \\
\hline Northeast & 0 & 0 & 0 & 0 & 0 & 1 & 0 & 0 & 0 & 0 & 0 & 0 \\
\hline Midwest & 0 & 0 & 0 & 0 & 0 & 0 & 0 & 0 & 0 & 0 & 0 & 0 \\
\hline South & 0 & 0 & 0 & 0 & 0 & 0 & 0 & 0 & 0 & 0 & 0 & 0 \\
\hline West & 1 & 0 & 1 & 0 & 0 & 0 & 1 & 0 & 0 & 0 & 1 & 0 \\
\hline \multirow[t]{2}{*}{ Total } & 1 & 0 & 1 & 0 & 0 & 1 & 1 & 0 & 0 & 0 & 1 & 0 \\
\hline & \multicolumn{12}{|c|}{ White } \\
\hline \multicolumn{13}{|c|}{ Age at diagnosis (yr) } \\
\hline $13-14$ & 0 & 0 & 0 & 0 & 0 & 0 & 0 & 0 & 0 & 0 & 0 & 0 \\
\hline $15-19$ & 0 & 0 & 0 & 1 & 0 & 0 & 0 & 1 & 0 & 0 & 2 & 0 \\
\hline $20-24$ & 7 & 9 & 2 & 7 & 7 & 5 & 8 & 2 & 3 & 12 & 6 & 4 \\
\hline $25-29$ & 13 & 17 & 8 & 11 & 18 & 20 & 23 & 20 & 15 & 22 & 22 & 23 \\
\hline 30-34 & 20 & 18 & 26 & 21 & 19 & 21 & 38 & 30 & 31 & 14 & 27 & 38 \\
\hline $35-39$ & 48 & 21 & 25 & 31 & 16 & 20 & 29 & 42 & 32 & 27 & 39 & 36 \\
\hline $40-44$ & 49 & 43 & 35 & 46 & 36 & 35 & 48 & 45 & 45 & 38 & 40 & 34 \\
\hline $45-49$ & 73 & 61 & 59 & 54 & 47 & 39 & 59 & 55 & 55 & 37 & 39 & 32 \\
\hline $50-54$ & 71 & 64 & 60 & 44 & 50 & 40 & 44 & 50 & 52 & 38 & 36 & 31 \\
\hline $55-59$ & 37 & 32 & 45 & 39 & 24 & 33 & 22 & 29 & 24 & 22 & 29 & 19 \\
\hline $60-64$ & 20 & 16 & 20 & 17 & 19 & 17 & 6 & 16 & 16 & 14 & 9 & 12 \\
\hline$\geq 65$ & 7 & 7 & 13 & 16 & 9 & 11 & 7 & 4 & 6 & 5 & 7 & 6 \\
\hline \multicolumn{13}{|c|}{ Region of residence ${ }^{a}$} \\
\hline Northeast & 79 & 47 & 60 & 48 & 45 & 48 & 65 & 72 & 54 & 43 & 42 & 41 \\
\hline Midwest & 37 & 38 & 25 & 34 & 30 & 28 & 38 & 34 & 39 & 21 & 39 & 36 \\
\hline South & 127 & 117 & 123 & 114 & 101 & 97 & 123 & 132 & 133 & 108 & 114 & 115 \\
\hline West & 102 & 85 & 85 & 90 & 67 & 67 & 58 & 54 & 52 & 55 & 61 & 44 \\
\hline Total & 345 & 287 & 293 & 286 & 243 & 240 & 284 & 292 & 278 & 227 & 256 & 236 \\
\hline
\end{tabular}


Table 9a. Stage 3 (AIDS) attributed to injection drug use, by selected characteristics, 2011-2016-United States (cont)

\begin{tabular}{|c|c|c|c|c|c|c|c|c|c|c|c|}
\hline \multicolumn{6}{|c|}{ Male } & \multicolumn{6}{|c|}{ Female } \\
\hline 2011 & 2012 & 2013 & 2014 & 2015 & 2016 & 2011 & 2012 & 2013 & 2014 & 2015 & 2016 \\
\hline No. & No. & No. & No. & No. & No. & No. & No. & No. & No. & No. & No. \\
\hline
\end{tabular}

Age at diagnosis (yr)

\begin{tabular}{|c|c|c|c|c|c|c|c|c|c|c|c|c|}
\hline $13-14$ & 0 & 0 & 0 & 0 & 0 & 0 & 0 & 0 & 0 & 0 & 0 & 0 \\
\hline 15-19 & 0 & 0 & 0 & 0 & 0 & 0 & 0 & 1 & 1 & 0 & 0 & 0 \\
\hline $20-24$ & 1 & 1 & 0 & 1 & 0 & 0 & 2 & 2 & 2 & 1 & 0 & 0 \\
\hline $25-29$ & 8 & 2 & 0 & 2 & 4 & 2 & 5 & 7 & 4 & 4 & 3 & 3 \\
\hline $30-34$ & 5 & 6 & 3 & 5 & 1 & 1 & 8 & 7 & 7 & 4 & 11 & 7 \\
\hline $35-39$ & 8 & 3 & 2 & 4 & 1 & 3 & 7 & 10 & 8 & 5 & 4 & 3 \\
\hline 40-44 & 5 & 7 & 11 & 2 & 2 & 3 & 11 & 13 & 12 & 6 & 9 & 2 \\
\hline $45-49$ & 9 & 15 & 7 & 6 & 9 & 4 & 19 & 16 & 8 & 9 & 7 & 8 \\
\hline $50-54$ & 18 & 14 & 13 & 9 & 7 & 9 & 14 & 9 & 9 & 10 & 11 & 10 \\
\hline $55-59$ & 15 & 13 & 4 & 14 & 6 & 5 & 6 & 5 & 9 & 4 & 7 & 7 \\
\hline $60-64$ & 2 & 10 & 6 & 4 & 4 & 6 & 2 & 2 & 4 & 4 & 4 & 2 \\
\hline$\geq 65$ & 4 & 5 & 7 & 4 & 5 & 8 & 2 & 2 & 2 & 3 & 1 & 0 \\
\hline \multicolumn{13}{|c|}{ Region of residence $\mathrm{a}^{\mathrm{a}}$} \\
\hline Northeast & 24 & 37 & 17 & 20 & 14 & 20 & 32 & 27 & 24 & 21 & 12 & 17 \\
\hline Midwest & 9 & 6 & 2 & 5 & 3 & 3 & 6 & 11 & 2 & 4 & 8 & 6 \\
\hline South & 32 & 24 & 26 & 17 & 18 & 14 & 34 & 26 & 34 & 17 & 27 & 16 \\
\hline West & 12 & 9 & 8 & 8 & 5 & 2 & 6 & 10 & 6 & 8 & 10 & 4 \\
\hline Total & 77 & 76 & 53 & 50 & 40 & 39 & 78 & 74 & 66 & 50 & 57 & 43 \\
\hline
\end{tabular}

Total

Age at diagnosis (yr)

\begin{tabular}{|c|c|c|c|c|c|c|c|c|c|c|c|c|}
\hline $13-14$ & 0 & 0 & 0 & 0 & 0 & 0 & 0 & 0 & 0 & 0 & 0 & 0 \\
\hline 15-19 & 1 & 3 & 2 & 2 & 0 & 2 & 5 & 5 & 3 & 3 & 3 & 1 \\
\hline $20-24$ & 26 & 27 & 19 & 16 & 18 & 14 & 33 & 21 & 16 & 27 & 21 & 14 \\
\hline $25-29$ & 78 & 64 & 43 & 43 & 55 & 53 & 81 & 66 & 55 & 56 & 52 & 43 \\
\hline 30-34 & 108 & 108 & 104 & 75 & 75 & 70 & 128 & 105 & 95 & 57 & 80 & 83 \\
\hline 35-39 & 163 & 130 & 106 & 127 & 85 & 98 & 141 & 148 & 118 & 91 & 96 & 105 \\
\hline 40-44 & 232 & 193 & 149 & 144 & 115 & 107 & 201 & 186 & 151 & 131 & 108 & 92 \\
\hline $45-49$ & 304 & 276 & 258 & 186 & 155 & 126 & 260 & 216 & 177 & 146 & 126 & 106 \\
\hline 50-54 & 327 & 319 & 265 & 194 & 190 & 149 & 219 & 198 & 175 & 154 & 123 & 110 \\
\hline $55-59$ & 238 & 227 & 196 & 187 & 144 & 148 & 135 & 129 & 127 & 101 & 91 & 87 \\
\hline 60-64 & 111 & 127 & 122 & 100 & 102 & 101 & 46 & 66 & 67 & 45 & 57 & 53 \\
\hline$\geq 65$ & 67 & 83 & 83 & 85 & 70 & 86 & 35 & 34 & 38 & 31 & 32 & 33 \\
\hline \multicolumn{13}{|c|}{ Region of residence ${ }^{a}$} \\
\hline Northeast & 569 & 498 & 446 & 342 & 298 & 299 & 400 & 354 & 285 & 248 & 217 & 180 \\
\hline Midwest & 152 & 148 & 111 & 92 & 102 & 85 & 139 & 138 & 116 & 93 & 85 & 97 \\
\hline South & 692 & 702 & 588 & 526 & 443 & 412 & 598 & 547 & 509 & 381 & 366 & 351 \\
\hline West & 243 & 209 & 203 & 200 & 166 & 157 & 148 & 136 & 113 & 118 & 118 & 100 \\
\hline Total & 1,657 & 1,557 & 1,348 & 1,159 & 1,010 & 952 & 1,285 & 1,176 & 1,023 & 840 & 786 & 728 \\
\hline
\end{tabular}

Note. Data for the year 2016 are preliminary (subject to change) because they are based on only a 6-month reporting delay. Data for the year 2016 should not be used when assessing trends. Numbers less than 12 , and trends based on these numbers, should be interpreted with caution. Data have been statistically adjusted to account for missing transmission category; therefore, values may not sum to column total.

Data are based on residence at time of stage 3 (AIDS) classification

${ }^{\mathrm{b}}$ Includes Asian/Pacific Islander legacy cases (see Technical Notes).

${ }^{\mathrm{C}}$ Hispanics/Latinos can be of any race. 
Table 9b. Stage 3 (AIDS) attributed to injection drug use, by selected characteristics, 2011-2016-United States and 6 dependent areas

\begin{tabular}{|c|c|c|c|c|c|c|c|c|c|c|c|}
\hline \multicolumn{6}{|c|}{ Male } & \multicolumn{6}{|c|}{ Female } \\
\hline 2011 & 2012 & 2013 & 2014 & 2015 & 2016 & 2011 & 2012 & 2013 & 2014 & 2015 & 2016 \\
\hline No. & No. & No. & No. & No. & No. & No. & No. & No. & No. & No. & No. \\
\hline
\end{tabular}

American Indian/Alaska Native

Age at diagnosis (yr)

$13-14$
$15-19$
$20-24$
$25-29$
$30-34$
$35-39$
$40-44$
$45-49$
$50-54$
$55-59$
$60-64$
$\geq 65$

$\begin{array}{llll}0 & 0 & 0 & 0 \\ 0 & 0 & 0 & 0 \\ 0 & 0 & 0 & 0 \\ 0 & 2 & 0 & 2 \\ 1 & 1 & 1 & 1 \\ 2 & 0 & 0 & 0 \\ 0 & 0 & 0 & 4 \\ 3 & 1 & 2 & 1 \\ 1 & 1 & 1 & 2 \\ 2 & 1 & 0 & 1 \\ 0 & 0 & 0 & 0 \\ 0 & 0 & 1 & 0\end{array}$

$\begin{array}{ll}0 & 0 \\ 0 & 0 \\ 0 & 0 \\ 0 & 0 \\ 2 & 0 \\ 0 & 0 \\ 1 & 1 \\ 0 & 3 \\ 2 & 0 \\ 1 & 1 \\ 0 & 0 \\ 0 & 0\end{array}$

$\begin{array}{ll}0 & 0 \\ 0 & 0 \\ 0 & 1 \\ 0 & 0 \\ 0 & 2 \\ 0 & 3 \\ 1 & 0 \\ 3 & 1 \\ 0 & 1 \\ 1 & 1 \\ 0 & 1 \\ 0 & 0\end{array}$

$\begin{array}{ll}0 & 0 \\ 0 & 0 \\ 0 & 0 \\ 0 & 1 \\ 2 & 1 \\ 2 & 1 \\ 0 & 0 \\ 1 & 1 \\ 0 & 1 \\ 4 & 0 \\ 0 & 1 \\ 0 & 0\end{array}$

$\begin{array}{lll}0 & 0 & 0 \\ 0 & 0 & 0 \\ 0 & 0 & 1 \\ 0 & 1 & 1 \\ 1 & 1 & 1 \\ 3 & 2 & 1 \\ 2 & 1 & 1 \\ 0 & 1 & 1 \\ 1 & 2 & 0 \\ 0 & 1 & 2 \\ 1 & 0 & 0 \\ 0 & 0 & 0\end{array}$

Region of residence ${ }^{a}$ Northeast

Midwest

South

West

U.S. dependent areas

$\begin{array}{llll}0 & 0 & 0 & 0 \\ 0 & 2 & 0 & 1 \\ 3 & 1 & 2 & 4 \\ 6 & 4 & 3 & 6 \\ 0 & 0 & 0 & 0 \\ 9 & 7 & 5 & 11\end{array}$

$\begin{array}{ll}0 & 1 \\ 0 & 0 \\ 0 & 1 \\ 5 & 5 \\ 0 & 0 \\ 5 & 7\end{array}$

\begin{tabular}{rrrrrr}
0 & 1 & 0 & 0 & 0 & 0 \\
4 & 3 & 0 & 0 & 2 & 1 \\
1 & 3 & 2 & 3 & 5 & 1 \\
6 & 3 & 4 & 5 & 4 & 6 \\
0 & 0 & 0 & 0 & 0 & 0 \\
11 & 10 & 6 & 8 & 11 & 8 \\
\hline
\end{tabular}

Total

Age at diagnosis (yr)

13-14

15-19

20-24

25-29

30-34

35-39

40-44

$45-49$

$50-54$

55-59

$60-64$

$\geq 65$

Region of residence ${ }^{a}$

Northeast

Midwest

South

West

U.S. dependent areas

Total

9

\section{$\begin{array}{llll}7 & 11 & 5 & 7 \\ & & \text { Asian } & \\ & & \end{array}$}

$\begin{array}{llllllllllllll}0 & 0 & 0 & 0 & 0 & 0 & 0 & 0 & 0 & 0 & 0 & 0 \\ 0 & 0 & 0 & 0 & 0 & 0 & 0 & 0 & 0 & 0 & 0 & 0 \\ 0 & 0 & 0 & 0 & 1 & 0 & 2 & 0 & 0 & 0 & 0 & 0 \\ 1 & 0 & 0 & 0 & 0 & 1 & 0 & 0 & 0 & 0 & 0 & 0 \\ 3 & 2 & 2 & 1 & 4 & 2 & 0 & 0 & 0 & 0 & 0 & 1 \\ 1 & 5 & 1 & 2 & 3 & 2 & 1 & 2 & 0 & 1 & 0 & 1 \\ 4 & 4 & 1 & 2 & 1 & 1 & 1 & 1 & 1 & 0 & 0 & 0 \\ 2 & 2 & 1 & 0 & 1 & 2 & 1 & 0 & 0 & 1 & 0 & 0 \\ 1 & 1 & 0 & 1 & 1 & 1 & 0 & 0 & 1 & 1 & 0 & 0 \\ 0 & 1 & 2 & 0 & 1 & 1 & 0 & 0 & 0 & 1 & 1 & 0 \\ 0 & 0 & 1 & 0 & 1 & 1 & 0 & 0 & 0 & 0 & 1 & 0 \\ 0 & 0 & 0 & 1 & 1 & 1 & 0 & 0 & 0 & 0 & 0 & 0\end{array}$


Table 9b. Stage 3 (AIDS) attributed to injection drug use, by selected characteristics, 2011-2016-United States and 6 dependent areas (cont)

\begin{tabular}{|c|c|c|c|c|c|c|c|c|c|c|c|c|}
\hline & \multicolumn{6}{|c|}{ Male } & \multicolumn{6}{|c|}{ Female } \\
\hline & 2011 & 2012 & 2013 & 2014 & 2015 & 2016 & 2011 & 2012 & 2013 & 2014 & 2015 & 2016 \\
\hline & No. & No. & No. & No. & No. & No. & No. & No. & No. & No. & No. & No. \\
\hline & \multicolumn{12}{|c|}{ Black/African American } \\
\hline \multicolumn{13}{|l|}{ Age at diagnosis (yr) } \\
\hline $13-14$ & 0 & 0 & 0 & 0 & 0 & 0 & 0 & 0 & 0 & 0 & 0 & 0 \\
\hline $15-19$ & 1 & 3 & 2 & 1 & 0 & 1 & 4 & 3 & 1 & 2 & 1 & 1 \\
\hline $20-24$ & 7 & 12 & 8 & 5 & 4 & 4 & 17 & 14 & 7 & 9 & 9 & 6 \\
\hline $25-29$ & 23 & 25 & 21 & 12 & 20 & 12 & 39 & 27 & 25 & 17 & 18 & 13 \\
\hline $30-34$ & 41 & 38 & 38 & 22 & 19 & 20 & 54 & 49 & 37 & 24 & 27 & 25 \\
\hline $35-39$ & 57 & 56 & 42 & 46 & 29 & 42 & 79 & 68 & 59 & 37 & 34 & 41 \\
\hline $40-44$ & 99 & 91 & 55 & 49 & 38 & 38 & 106 & 97 & 64 & 65 & 39 & 42 \\
\hline $45-49$ & 139 & 118 & 118 & 80 & 60 & 47 & 145 & 110 & 83 & 74 & 54 & 45 \\
\hline $50-54$ & 169 & 169 & 141 & 87 & 91 & 67 & 121 & 114 & 89 & 80 & 55 & 50 \\
\hline $55-59$ & 135 & 141 & 109 & 90 & 84 & 76 & 85 & 75 & 81 & 57 & 43 & 50 \\
\hline $60-64$ & 67 & 71 & 71 & 65 & 62 & 58 & 29 & 37 & 35 & 20 & 34 & 34 \\
\hline$\geq 65$ & 40 & 56 & 50 & 51 & 40 & 41 & 20 & 25 & 21 & 20 & 15 & 20 \\
\hline \multicolumn{13}{|l|}{ Region of residence ${ }^{a}$} \\
\hline Northeast & 238 & 217 & 197 & 139 & 124 & 117 & 190 & 162 & 112 & 113 & 93 & 70 \\
\hline Midwest & 74 & 82 & 68 & 39 & 51 & 41 & 81 & 79 & 69 & 60 & 29 & 46 \\
\hline South & 423 & 440 & 351 & 302 & 251 & 220 & 383 & 338 & 293 & 212 & 179 & 187 \\
\hline West & 42 & 38 & 39 & 26 & 21 & 27 & 46 & 38 & 27 & 21 & 27 & 23 \\
\hline U.S. dependent areas & 1 & 1 & 0 & 1 & 1 & 0 & 0 & 0 & 0 & 0 & 0 & 0 \\
\hline Total & 778 & 778 & 655 & 507 & 448 & 405 & 700 & 617 & 501 & 406 & 328 & 326 \\
\hline & \multicolumn{12}{|c|}{ Hispanic/Latino ${ }^{c}$} \\
\hline \multicolumn{13}{|l|}{ Age at diagnosis (yr) } \\
\hline $13-14$ & 0 & 0 & 0 & 0 & 0 & 0 & 0 & 0 & 0 & 0 & 0 & 0 \\
\hline $15-19$ & 0 & 0 & 0 & 0 & 0 & 1 & 0 & 0 & 2 & 0 & 0 & 0 \\
\hline $20-24$ & 12 & 5 & 11 & 2 & 6 & 6 & 3 & 4 & 5 & 5 & 6 & 3 \\
\hline $25-29$ & 38 & 21 & 17 & 17 & 14 & 18 & 15 & 12 & 12 & 14 & 8 & 3 \\
\hline $30-34$ & 48 & 53 & 42 & 28 & 34 & 28 & 31 & 20 & 20 & 21 & 14 & 13 \\
\hline $35-39$ & 63 & 58 & 47 & 59 & 43 & 33 & 27 & 27 & 22 & 22 & 20 & 25 \\
\hline $40-44$ & 90 & 66 & 54 & 49 & 42 & 34 & 39 & 33 & 29 & 24 & 18 & 13 \\
\hline $45-49$ & 105 & 98 & 83 & 59 & 47 & 39 & 38 & 42 & 42 & 28 & 26 & 23 \\
\hline $50-54$ & 82 & 84 & 62 & 64 & 48 & 40 & 44 & 29 & 28 & 29 & 19 & 19 \\
\hline $55-59$ & 56 & 52 & 49 & 54 & 36 & 38 & 22 & 20 & 18 & 21 & 9 & 11 \\
\hline $60-64$ & 22 & 30 & 28 & 17 & 19 & 23 & 8 & 14 & 13 & 6 & 9 & 5 \\
\hline$\geq 65$ & 21 & 22 & 15 & 15 & 16 & 28 & 6 & 3 & 9 & 3 & 9 & 7 \\
\hline \multicolumn{13}{|l|}{ Region of residence ${ }^{a}$} \\
\hline Northeast & 225 & 193 & 170 & 132 & 111 & 108 & 113 & 90 & 96 & 71 & 71 & 51 \\
\hline Midwest & 30 & 20 & 16 & 13 & 18 & 10 & 10 & 12 & 6 & 7 & 6 & 7 \\
\hline South & 104 & 115 & 82 & 84 & 71 & 78 & 58 & 47 & 47 & 39 & 40 & 32 \\
\hline West & 75 & 66 & 64 & 67 & 60 & 52 & 27 & 31 & 23 & 27 & 15 & 23 \\
\hline U.S. dependent areas & 102 & 94 & 76 & 68 & 45 & 40 & 26 & 24 & 28 & 28 & 8 & 8 \\
\hline Total & 536 & 488 & 408 & 364 & 305 & 288 & 234 & 204 & 200 & 172 & 140 & 121 \\
\hline
\end{tabular}


Table 9b. Stage 3 (AIDS) attributed to injection drug use, by selected characteristics, 2011-2016-United States and 6 dependent areas (cont)

\begin{tabular}{|c|c|c|c|c|c|c|c|c|c|c|c|}
\hline \multicolumn{6}{|c|}{ Male } & \multicolumn{6}{|c|}{ Female } \\
\hline 2011 & 2012 & 2013 & 2014 & 2015 & 2016 & 2011 & 2012 & 2013 & 2014 & 2015 & 2016 \\
\hline No. & No. & No. & No. & No. & No. & No. & No. & No. & No. & No. & No. \\
\hline
\end{tabular}

Age at diagnosis (yr)

$\begin{array}{lllllllllllll}13-14 & 0 & 0 & 0 & 0 & 0 & 0 & 0 & 0 & 0 & 0 & 0 & 0 \\ 15-19 & 0 & 0 & 0 & 0 & 0 & 0 & 0 & 0 & 0 & 0 & 0 & 0 \\ 20-24 & 0 & 0 & 0 & 0 & 0 & 0 & 0 & 0 & 0 & 0 & 0 & 0 \\ 25-29 & 0 & 0 & 0 & 0 & 0 & 0 & 0 & 0 & 0 & 0 & 0 & 0 \\ 30-34 & 0 & 0 & 0 & 0 & 0 & 0 & 0 & 0 & 0 & 0 & 0 & 0 \\ 35-39 & 1 & 0 & 0 & 0 & 0 & 1 & 1 & 0 & 0 & 0 & 0 & 0 \\ 40-44 & 0 & 0 & 0 & 0 & 0 & 0 & 0 & 0 & 0 & 0 & 0 & 0 \\ 45-49 & 0 & 0 & 1 & 0 & 0 & 0 & 0 & 0 & 0 & 0 & 0 & 0 \\ 50-54 & 0 & 0 & 0 & 0 & 0 & 0 & 0 & 0 & 0 & 0 & 0 & 0 \\ 55-59 & 0 & 0 & 0 & 0 & 0 & 0 & 0 & 0 & 0 & 0 & 1 & 0 \\ 60-64 & 0 & 0 & 0 & 0 & 0 & 0 & 0 & 0 & 0 & 0 \\ \geq 65 & 0 & 0 & 0 & 0 & 0 & 0 & 0 & 0 & 0 & 0\end{array}$

Region of residence ${ }^{a}$

Northeas

Midwest

South

West

U.S. dependent areas

Total

0
0
0
1
0
1

$\begin{array}{ll}0 & 0 \\ 0 & 0 \\ 0 & 0 \\ 0 & 1 \\ 0 & 0 \\ 0 & 1\end{array}$

0
0
0
0
0
0

$\begin{array}{llll}0 & 1 & 0 & \\ 0 & 0 & 0 & \\ 0 & 0 & 0 & 0 \\ 0 & 0 & 1 & 0 \\ 0 & 0 & 0 & 0 \\ 0 & 1 & 1 & 0\end{array}$

0
0
0
0
0
0

\section{White}

Age at diagnosis (yr)

\begin{tabular}{|c|c|c|c|c|c|c|c|c|c|c|c|c|}
\hline & & & & & & & & & & & & \\
\hline $13-14$ & 0 & 0 & 0 & 0 & 0 & 0 & 0 & 0 & 0 & 0 & 0 & 0 \\
\hline $15-19$ & 0 & 0 & 0 & 1 & 0 & 0 & 0 & 1 & 0 & 0 & 2 & 0 \\
\hline 20-24 & 7 & 9 & 2 & 7 & 7 & 5 & 8 & 2 & 3 & 12 & 6 & 4 \\
\hline $25-29$ & 13 & 17 & 8 & 11 & 18 & 20 & 23 & 20 & 15 & 22 & 22 & 23 \\
\hline 30-34 & 20 & 18 & 26 & 21 & 19 & 21 & 38 & 30 & 31 & 14 & 27 & 38 \\
\hline $35-39$ & 48 & 21 & 25 & 31 & 16 & 20 & 29 & 42 & 32 & 27 & 39 & 36 \\
\hline 40-44 & 49 & 43 & 35 & 46 & 36 & 35 & 48 & 45 & 45 & 38 & 40 & 34 \\
\hline $45-49$ & 73 & 61 & 59 & 54 & 47 & 39 & 59 & 55 & 55 & 37 & 39 & 32 \\
\hline $50-54$ & 71 & 64 & 60 & 44 & 50 & 40 & 44 & 50 & 52 & 38 & 36 & 31 \\
\hline $55-59$ & 37 & 32 & 45 & 39 & 24 & 33 & 22 & 29 & 24 & 22 & 29 & 19 \\
\hline $60-64$ & 20 & 16 & 20 & 17 & 19 & 17 & 6 & 16 & 16 & 14 & 9 & 12 \\
\hline$\geq 65$ & 7 & 7 & 13 & 16 & 9 & 11 & 7 & 4 & 6 & 5 & 7 & 6 \\
\hline \multicolumn{13}{|l|}{ Region of residence $^{a}$} \\
\hline Northeast & 79 & 47 & 60 & 48 & 45 & 48 & 65 & 72 & 54 & 43 & 42 & 41 \\
\hline Midwest & 37 & 38 & 25 & 34 & 30 & 28 & 38 & 34 & 39 & 21 & 39 & 36 \\
\hline South & 127 & 117 & 123 & 114 & 101 & 97 & 123 & 132 & 133 & 108 & 114 & 115 \\
\hline West & 102 & 85 & 85 & 90 & 67 & 67 & 58 & 54 & 52 & 55 & 61 & 44 \\
\hline U.S. dependent areas & 0 & 0 & 0 & 0 & 0 & 0 & 0 & 0 & 0 & 0 & 0 & 0 \\
\hline Total & 345 & 287 & 293 & 286 & 243 & 240 & 284 & 292 & 278 & 227 & 256 & 236 \\
\hline
\end{tabular}


Table 9b. Stage 3 (AIDS) attributed to injection drug use, by selected characteristics, 2011-2016-United States and 6 dependent areas (cont)

\begin{tabular}{|c|c|c|c|c|c|c|c|c|c|c|c|c|}
\hline & \multicolumn{6}{|c|}{ Male } & \multicolumn{6}{|c|}{ Female } \\
\hline & 2011 & 2012 & 2013 & 2014 & 2015 & 2016 & 2011 & 2012 & 2013 & 2014 & 2015 & 2016 \\
\hline & No. & No. & No. & No. & No. & No. & No. & No. & No. & No. & No. & No. \\
\hline & \multicolumn{12}{|c|}{ Multiple races } \\
\hline \multicolumn{13}{|l|}{ Age at diagnosis (yr) } \\
\hline $13-14$ & 0 & 0 & 0 & 0 & 0 & 0 & 0 & 0 & 0 & 0 & 0 & 0 \\
\hline $15-19$ & 0 & 0 & 0 & 0 & 0 & 0 & 0 & 1 & 1 & 0 & 0 & 0 \\
\hline $20-24$ & 1 & 1 & 0 & 1 & 0 & 0 & 2 & 2 & 2 & 1 & 0 & 0 \\
\hline $25-29$ & 8 & 2 & 0 & 2 & 4 & 2 & 5 & 7 & 4 & 4 & 3 & 3 \\
\hline $30-34$ & 5 & 6 & 3 & 5 & 1 & 1 & 8 & 7 & 7 & 4 & 11 & 7 \\
\hline $35-39$ & 8 & 3 & 2 & 4 & 1 & 3 & 7 & 10 & 8 & 5 & 4 & 3 \\
\hline $40-44$ & 5 & 7 & 11 & 2 & 2 & 3 & 11 & 13 & 12 & 6 & 9 & 2 \\
\hline $45-49$ & 9 & 15 & 7 & 6 & 9 & 4 & 19 & 16 & 8 & 9 & 7 & 8 \\
\hline $50-54$ & 18 & 14 & 13 & 9 & 7 & 9 & 14 & 9 & 9 & 10 & 11 & 10 \\
\hline $55-59$ & 15 & 13 & 4 & 14 & 6 & 5 & 6 & 5 & 9 & 4 & 7 & 7 \\
\hline $60-64$ & 2 & 10 & 6 & 4 & 4 & 6 & 2 & 2 & 4 & 4 & 4 & 2 \\
\hline$\geq 65$ & 4 & 5 & 7 & 4 & 5 & 8 & 2 & 2 & 2 & 3 & 1 & 0 \\
\hline \multicolumn{13}{|l|}{ Region of residence ${ }^{a}$} \\
\hline Northeast & 24 & 37 & 17 & 20 & 14 & 20 & 32 & 27 & 24 & 21 & 12 & 17 \\
\hline Midwest & 9 & 6 & 2 & 5 & 3 & 3 & 6 & 11 & 2 & 4 & 8 & 6 \\
\hline South & 32 & 24 & 26 & 17 & 18 & 14 & 34 & 26 & 34 & 17 & 27 & 16 \\
\hline West & 12 & 9 & 8 & 8 & 5 & 2 & 6 & 10 & 6 & 8 & 10 & 4 \\
\hline U.S. dependent areas & 0 & 0 & 0 & 0 & 0 & 0 & 0 & 0 & 0 & 0 & 0 & 0 \\
\hline \multirow[t]{2}{*}{ Total } & 77 & 76 & 53 & 50 & 40 & 39 & 78 & 74 & 66 & 50 & 57 & 43 \\
\hline & \multicolumn{12}{|c|}{ Total } \\
\hline \multicolumn{13}{|l|}{ Age at diagnosis (yr) } \\
\hline $13-14$ & 0 & 0 & 0 & 0 & 0 & 0 & 0 & 0 & 0 & 0 & 0 & 0 \\
\hline $15-19$ & 1 & 3 & 2 & 2 & 0 & 2 & 5 & 5 & 3 & 3 & 3 & 1 \\
\hline 20-24 & 28 & 28 & 22 & 16 & 18 & 15 & 33 & 21 & 16 & 27 & 21 & 14 \\
\hline 25-29 & 83 & 66 & 47 & 44 & 56 & 53 & 82 & 66 & 57 & 57 & 52 & 43 \\
\hline 30-34 & 118 & 118 & 112 & 78 & 79 & 73 & 133 & 108 & 95 & 64 & 81 & 84 \\
\hline $35-39$ & 180 & 143 & 117 & 142 & 92 & 102 & 146 & 151 & 122 & 94 & 99 & 107 \\
\hline $40-44$ & 247 & 210 & 156 & 152 & 120 & 111 & 205 & 189 & 152 & 136 & 108 & 92 \\
\hline $45-49$ & 331 & 295 & 271 & 200 & 164 & 132 & 263 & 223 & 190 & 150 & 127 & 110 \\
\hline $50-54$ & 342 & 333 & 278 & 208 & 199 & 158 & 225 & 201 & 179 & 158 & 124 & 110 \\
\hline $55-59$ & 245 & 239 & 208 & 198 & 151 & 154 & 136 & 132 & 130 & 105 & 91 & 89 \\
\hline $60-64$ & 112 & 127 & 125 & 103 & 105 & 105 & 46 & 68 & 68 & 45 & 58 & 53 \\
\hline$\geq 65$ & 71 & 90 & 85 & 87 & 71 & 89 & 36 & 34 & 38 & 31 & 33 & 33 \\
\hline \multicolumn{13}{|l|}{ Region of residence ${ }^{a}$} \\
\hline Northeast & 569 & 498 & 446 & 342 & 298 & 299 & 400 & 354 & 285 & 248 & 217 & 180 \\
\hline Midwest & 152 & 148 & 111 & 92 & 102 & 85 & 139 & 138 & 116 & 93 & 85 & 97 \\
\hline South & 692 & 702 & 588 & 526 & 443 & 412 & 598 & 547 & 509 & 381 & 366 & 351 \\
\hline West & 243 & 209 & 203 & 200 & 166 & 157 & 148 & 136 & 113 & 118 & 118 & 100 \\
\hline U.S. dependent areas & 103 & 95 & 76 & 70 & 46 & 41 & 26 & 24 & 28 & 29 & 8 & 8 \\
\hline Total & 1,759 & 1,652 & 1,424 & 1,229 & 1,057 & 993 & 1,311 & 1,200 & 1,051 & 869 & 794 & 736 \\
\hline
\end{tabular}

Note. Data for the year 2016 are preliminary (subject to change) because they are based on only a 6-month reporting delay. Data for the year 2016 should not be used when assessing trends. Numbers less than 12 , and trends based on these numbers, should be interpreted with caution. Data have been statistically adjusted to account for missing transmission category; therefore, values may not sum to column total.

a Data are based on residence at time of stage 3 (AIDS) classification.



${ }^{\mathrm{C}}$ Hispanics/Latinos can be of any race. 
Table 10a. Stage 3 (AIDS) attributed to heterosexual contact, by selected characteristics, 2011-2016-United States

\begin{tabular}{|c|c|c|c|c|c|c|c|c|c|c|c|}
\hline \multicolumn{6}{|c|}{ Male } & \multicolumn{6}{|c|}{ Female } \\
\hline 2011 & 2012 & 2013 & 2014 & 2015 & 2016 & 2011 & 2012 & 2013 & 2014 & 2015 & 2016 \\
\hline No. & No. & No. & No. & No. & No. & No. & No. & No. & No. & No. & No. \\
\hline
\end{tabular}

American Indian/Alaska Native

Age at diagnosis (yr)

$13-14$
$15-19$
$20-24$
$25-29$
$30-34$
$35-39$
$40-44$
$45-49$
$50-54$
$55-59$
$60-64$
$\geq 65$

$\begin{array}{llll}0 & 0 & 0 & 0 \\ 1 & 0 & 0 & 0 \\ 1 & 0 & 0 & 0 \\ 0 & 0 & 1 & 0 \\ 1 & 1 & 2 & 1 \\ 0 & 0 & 3 & 0 \\ 1 & 0 & 0 & 1 \\ 0 & 1 & 3 & 0 \\ 1 & 0 & 0 & 0 \\ 0 & 2 & 0 & 1 \\ 1 & 1 & 1 & 0 \\ 1 & 0 & 0 & 0\end{array}$

$\begin{array}{ll}0 & 0 \\ 0 & 0 \\ 0 & 0 \\ 1 & 0 \\ 2 & 1 \\ 0 & 1 \\ 0 & 1 \\ 1 & 1 \\ 1 & 0 \\ 0 & 0 \\ 0 & 0 \\ 0 & 1\end{array}$

$\begin{array}{ll}0 & 0 \\ 0 & 1 \\ 0 & 0 \\ 0 & 2 \\ 1 & 4 \\ 1 & 3 \\ 1 & 2 \\ 1 & 0 \\ 0 & 3 \\ 0 & 1 \\ 0 & 0 \\ 1 & 0\end{array}$

$\begin{array}{lllll}0 & 0 & 0 & 0 & 0 \\ 0 & 0 & 0 & 0 & 0 \\ 1 & 0 & 0 & 2 & 0 \\ 0 & 2 & 1 & 6 & 1 \\ 3 & 3 & 2 & 3 & 2 \\ 2 & 0 & 1 & 4 & 5 \\ 1 & 1 & 2 & 3 & 4 \\ 2 & 1 & 0 & 3 & 1 \\ 0 & 4 & 4 & 4 & 1 \\ 2 & 2 & 1 & 4 & 2 \\ 2 & 0 & 2 & 1 & 0 \\ 1 & 3 & 0 & 0 & 0\end{array}$

Region of residence ${ }^{a}$ Northeast Midwest

South

West

\begin{tabular}{rrrrrrrrrrrrr}
0 & 1 & 1 & 1 & 0 & 0 & 0 & 1 & 0 & 1 & 0 & 1 \\
1 & 1 & 6 & 0 & 0 & 1 & 4 & 2 & 4 & 2 & 6 & 6 \\
2 & 0 & 1 & 0 & 1 & 1 & 3 & 1 & 4 & 2 & 17 & 3 \\
4 & 4 & 4 & 2 & 5 & 4 & 8 & 8 & 6 & 8 & 5 & 6 \\
7 & 6 & 12 & 3 & 6 & 6 & 15 & 12 & 14 & 13 & 28 & 16 \\
\hline
\end{tabular}

Total

7

Age at diagnosis (yr)

$13-14$
$15-19$
$20-24$
$25-29$
$30-34$
$35-39$
$40-44$
$45-49$
$50-54$
$55-59$
$60-64$
$\geq 65$

$\begin{array}{ll}0 & 0 \\ 0 & 0 \\ 0 & 0 \\ 0 & 0 \\ 3 & 3 \\ 5 & 2 \\ 8 & 7 \\ 8 & 4 \\ 2 & 7 \\ 1 & 2 \\ 4 & 6 \\ 5 & 0\end{array}$

Region of residence ${ }^{a}$

Northeast

Midwest

South

West

$\begin{array}{rrrr}6 & 12 & 6 & 3 \\ 8 & 7 & 2 & 8 \\ 6 & 6 & 8 & 10 \\ 14 & 7 & 13 & 10 \\ 34 & 32 & 29 & 31\end{array}$

3
8
10
10
31

$\begin{array}{rr}5 & 5 \\ 6 & 5 \\ 4 & 10 \\ 5 & 5 \\ 20 & 25\end{array}$

Asian ${ }^{\mathrm{b}}$

Total

$\begin{array}{lllll}0 & 0 & 0 & 0 & \\ 0 & 0 & 0 & 0 & \\ 0 & 0 & 0 & 0 & \\ 4 & 2 & 2 & 1 & 1 \\ 3 & 5 & 2 & 2 & \\ 5 & 5 & 4 & 7 & 1 \\ 5 & 6 & 5 & 2 & \\ 4 & 5 & 2 & 4 & \\ 5 & 5 & 2 & 4 & \\ 2 & 2 & 1 & 3 & \\ 2 & 0 & 0 & 1 & \\ 0 & 2 & 2 & 2\end{array}$

0
1
2
10
6
12
9
5
6
5
3
1

$\begin{array}{rrrrr}0 & 0 & 0 & 0 & 0 \\ 2 & 1 & 0 & 0 & 0 \\ 3 & 0 & 5 & 2 & 2 \\ 6 & 5 & 8 & 4 & 3 \\ 8 & 6 & 5 & 7 & 9 \\ 12 & 14 & 12 & 6 & 18 \\ 12 & 9 & 5 & 8 & 8 \\ 5 & 5 & 7 & 8 & 4 \\ 4 & 1 & 3 & 1 & 3 \\ 0 & 2 & 6 & 5 & 6 \\ 4 & 2 & 2 & 4 & 3 \\ 0 & 3 & 1 & 3 & 2\end{array}$

$\begin{array}{rr}10 & 11 \\ 7 & 8 \\ 16 & 11 \\ 24 & 24 \\ 57 & 54\end{array}$

$\begin{array}{rr}11 & 11 \\ 8 & 9 \\ 11 & 11 \\ 24 & 16 \\ \mathbf{5 4} & 47\end{array}$

$\begin{array}{rr}11 & \\ 9 & \\ 11 & 17 \\ 16 & 24 \\ 47 & 55\end{array}$

$\begin{array}{rrr}7 & 6 & 16 \\ 7 & 9 & 8 \\ 17 & 14 & 13 \\ 24 & 17 & 19 \\ 55 & 46 & 56\end{array}$


Table 10a. Stage 3 (AIDS) attributed to heterosexual contact, by selected characteristics, 2011-2016-United States (cont)

\begin{tabular}{|c|c|c|c|c|c|c|c|c|c|c|c|c|}
\hline & \multicolumn{6}{|c|}{ Male } & \multicolumn{6}{|c|}{ Female } \\
\hline & 2011 & 2012 & 2013 & 2014 & 2015 & 2016 & 2011 & 2012 & 2013 & 2014 & 2015 & 2016 \\
\hline & No. & No. & No. & No. & No. & No. & No. & No. & No. & No. & No. & No. \\
\hline & \multicolumn{12}{|c|}{ Black/African American } \\
\hline \multicolumn{13}{|c|}{ Age at diagnosis (yr) } \\
\hline $13-14$ & 1 & 0 & 0 & 1 & 0 & 0 & 1 & 1 & 0 & 1 & 2 & 0 \\
\hline $15-19$ & 4 & 7 & 8 & 3 & 3 & 6 & 54 & 41 & 37 & 19 & 20 & 16 \\
\hline $20-24$ & 37 & 47 & 48 & 33 & 30 & 37 & 197 & 154 & 157 & 106 & 107 & 105 \\
\hline $25-29$ & 83 & 89 & 102 & 75 & 64 & 86 & 317 & 306 & 302 & 239 & 241 & 207 \\
\hline $30-34$ & 164 & 150 & 152 & 122 & 113 & 116 & 493 & 479 & 391 & 337 & 325 & 269 \\
\hline $35-39$ & 215 & 198 & 168 & 155 & 129 & 141 & 507 & 499 & 423 & 372 & 349 & 349 \\
\hline $40-44$ & 293 & 279 & 229 & 182 & 190 & 169 & 493 & 508 & 451 & 373 & 335 & 321 \\
\hline $45-49$ & 375 & 334 & 260 & 219 & 196 & 179 & 511 & 468 & 464 & 352 & 335 & 276 \\
\hline $50-54$ & 278 & 277 & 237 & 223 & 179 & 187 & 352 & 372 & 344 & 294 & 263 & 250 \\
\hline $55-59$ & 188 & 168 & 174 & 160 & 176 & 156 & 229 & 249 & 251 & 211 & 189 & 188 \\
\hline $60-64$ & 113 & 108 & 120 & 89 & 90 & 106 & 138 & 148 & 129 & 132 & 121 & 127 \\
\hline$\geq 65$ & 115 & 114 & 109 & 107 & 92 & 111 & 103 & 95 & 88 & 105 & 113 & 93 \\
\hline \multicolumn{13}{|c|}{ Region of residence ${ }^{a}$} \\
\hline Northeast & 362 & 324 & 296 & 262 & 244 & 217 & 603 & 576 & 500 & 430 & 386 & 307 \\
\hline Midwest & 164 & 159 & 157 & 112 & 112 & 129 & 362 & 325 & 287 & 291 & 227 & 257 \\
\hline South & 1,277 & 1,233 & 1,097 & 950 & 850 & 891 & 2,275 & 2,264 & 2,131 & 1,707 & 1,660 & 1,520 \\
\hline West & 63 & 53 & 57 & 44 & 54 & 56 & 155 & 156 & 119 & 113 & 125 & 119 \\
\hline \multirow[t]{2}{*}{ Total } & 1,866 & 1,769 & 1,607 & 1,368 & 1,260 & 1,293 & 3,395 & 3,321 & 3,037 & 2,541 & 2,398 & 2,203 \\
\hline & \multicolumn{12}{|c|}{ Hispanic/Latino $^{c}$} \\
\hline \multicolumn{13}{|c|}{ Age at diagnosis (yr) } \\
\hline $13-14$ & 0 & 0 & 0 & 0 & 0 & 0 & 1 & 0 & 0 & 1 & 0 & 1 \\
\hline $15-19$ & 0 & 1 & 0 & 0 & 0 & 0 & 9 & 2 & 8 & 7 & 2 & 5 \\
\hline $20-24$ & 9 & 11 & 16 & 8 & 10 & 16 & 35 & 33 & 36 & 15 & 22 & 28 \\
\hline $25-29$ & 51 & 27 & 42 & 40 & 27 & 30 & 87 & 67 & 58 & 46 & 50 & 50 \\
\hline $30-34$ & 71 & 72 & 86 & 48 & 64 & 46 & 108 & 91 & 93 & 79 & 65 & 70 \\
\hline $35-39$ & 79 & 72 & 77 & 64 & 47 & 55 & 109 & 99 & 102 & 97 & 86 & 81 \\
\hline $40-44$ & 73 & 74 & 69 & 63 & 59 & 51 & 131 & 103 & 100 & 86 & 79 & 71 \\
\hline $45-49$ & 74 & 77 & 71 & 64 & 60 & 66 & 124 & 99 & 93 & 77 & 74 & 63 \\
\hline 50-54 & 56 & 58 & 64 & 48 & 56 & 47 & 69 & 79 & 89 & 54 & 68 & 71 \\
\hline $55-59$ & 40 & 44 & 41 & 37 & 32 & 33 & 42 & 56 & 37 & 50 & 37 & 55 \\
\hline $60-64$ & 23 & 22 & 21 & 23 & 19 & 20 & 28 & 38 & 33 & 26 & 29 & 22 \\
\hline$\geq 65$ & 26 & 26 & 28 & 22 & 22 & 20 & 29 & 33 & 29 & 17 & 18 & 38 \\
\hline \multicolumn{13}{|c|}{ Region of residence $^{a}$} \\
\hline Northeast & 165 & 169 & 152 & 131 & 107 & 101 & 304 & 269 & 255 & 194 & 170 & 212 \\
\hline Midwest & 40 & 32 & 27 & 19 & 30 & 25 & 41 & 42 & 36 & 32 & 26 & 32 \\
\hline South & 238 & 219 & 255 & 202 & 205 & 202 & 310 & 278 & 276 & 229 & 233 & 213 \\
\hline West & 60 & 63 & 80 & 64 & 54 & 55 & 117 & 111 & 110 & 100 & 100 & 99 \\
\hline Total & 503 & 483 & 514 & 416 & 396 & 383 & 772 & 700 & 677 & 555 & 529 & 556 \\
\hline
\end{tabular}


Table 10a. Stage 3 (AIDS) attributed to heterosexual contact, by selected characteristics, 2011-2016-United States (cont)

\begin{tabular}{|c|c|c|c|c|c|c|c|c|c|c|c|c|}
\hline & \multicolumn{6}{|c|}{ Male } & \multicolumn{6}{|c|}{ Female } \\
\hline & 2011 & 2012 & 2013 & 2014 & 2015 & 2016 & 2011 & 2012 & 2013 & 2014 & 2015 & 2016 \\
\hline & No. & No. & No. & No. & No. & No. & No. & No. & No. & No. & No. & No. \\
\hline & \multicolumn{12}{|c|}{ Native Hawaiian/Other Pacific Islander } \\
\hline \multicolumn{13}{|c|}{ Age at diagnosis (yr) } \\
\hline $13-14$ & 0 & 0 & 0 & 0 & 0 & 0 & 0 & 0 & 0 & 0 & 0 & 0 \\
\hline $15-19$ & 0 & 0 & 0 & 0 & 0 & 0 & 0 & 0 & 0 & 0 & 0 & 0 \\
\hline $20-24$ & 0 & 0 & 0 & 0 & 0 & 0 & 0 & 0 & 1 & 0 & 0 & 0 \\
\hline $25-29$ & 0 & 0 & 0 & 0 & 0 & 0 & 1 & 0 & 0 & 0 & 0 & 0 \\
\hline $30-34$ & 0 & 0 & 0 & 0 & 0 & 0 & 0 & 1 & 0 & 1 & 1 & 1 \\
\hline $35-39$ & 1 & 0 & 0 & 0 & 1 & 1 & 1 & 0 & 0 & 0 & 1 & 1 \\
\hline 40-44 & 0 & 0 & 0 & 2 & 0 & 0 & 0 & 0 & 0 & 0 & 0 & 0 \\
\hline $45-49$ & 0 & 0 & 0 & 0 & 1 & 1 & 1 & 1 & 2 & 0 & 0 & 0 \\
\hline $50-54$ & 0 & 0 & 0 & 0 & 1 & 0 & 0 & 1 & 2 & 0 & 0 & 1 \\
\hline $55-59$ & 0 & 0 & 0 & 0 & 0 & 0 & 1 & 0 & 0 & 1 & 0 & 0 \\
\hline $60-64$ & 0 & 0 & 0 & 0 & 0 & 0 & 0 & 0 & 0 & 1 & 0 & 0 \\
\hline$\geq 65$ & 0 & 0 & 1 & 0 & 0 & 0 & 0 & 0 & 0 & 0 & 0 & 0 \\
\hline \multicolumn{13}{|c|}{ Region of residence ${ }^{a}$} \\
\hline Northeast & 0 & 0 & 0 & 0 & 0 & 0 & 0 & 0 & 0 & 0 & 0 & 0 \\
\hline Midwest & 0 & 0 & 0 & 0 & 0 & 1 & 0 & 0 & 0 & 0 & 1 & 0 \\
\hline South & 0 & 0 & 0 & 1 & 0 & 1 & 0 & 0 & 3 & 1 & 0 & 0 \\
\hline West & 1 & 1 & 1 & 1 & 3 & 0 & 4 & 3 & 2 & 2 & 1 & 3 \\
\hline \multirow[t]{2}{*}{ Total } & 1 & 1 & 1 & 2 & 3 & 2 & 4 & 3 & 5 & 3 & 2 & 3 \\
\hline & \multicolumn{12}{|c|}{ White } \\
\hline \multicolumn{13}{|c|}{ Age at diagnosis (yr) } \\
\hline $13-14$ & 0 & 0 & 0 & 0 & 0 & 0 & 0 & 0 & 0 & 0 & 0 & 0 \\
\hline $15-19$ & 0 & 0 & 0 & 0 & 0 & 0 & 3 & 5 & 2 & 2 & 0 & 2 \\
\hline $20-24$ & 5 & 6 & 4 & 3 & 1 & 3 & 20 & 13 & 21 & 16 & 14 & 20 \\
\hline $25-29$ & 14 & 14 & 14 & 14 & 17 & 13 & 49 & 39 & 41 & 41 & 37 & 37 \\
\hline 30-34 & 12 & 21 & 18 & 15 & 15 & 20 & 89 & 79 & 58 & 57 & 53 & 45 \\
\hline $35-39$ & 34 & 23 & 30 & 19 & 18 & 14 & 93 & 77 & 68 & 69 & 59 & 70 \\
\hline $40-44$ & 46 & 44 & 47 & 35 & 23 & 31 & 92 & 101 & 80 & 74 & 59 & 59 \\
\hline $45-49$ & 60 & 48 & 44 & 41 & 41 & 29 & 87 & 96 & 90 & 66 & 73 & 64 \\
\hline 50-54 & 48 & 59 & 45 & 52 & 37 & 48 & 63 & 71 & 75 & 68 & 84 & 64 \\
\hline $55-59$ & 33 & 30 & 40 & 32 & 33 & 28 & 45 & 64 & 59 & 60 & 58 & 61 \\
\hline $60-64$ & 34 & 22 & 26 & 26 & 27 & 25 & 33 & 29 & 44 & 15 & 28 & 31 \\
\hline$\geq 65$ & 35 & 20 & 31 & 25 & 26 & 28 & 12 & 14 & 16 & 23 & 26 & 25 \\
\hline \multicolumn{13}{|c|}{ Region of residence $^{a}$} \\
\hline Northeast & 79 & 65 & 63 & 64 & 49 & 42 & 94 & 99 & 86 & 71 & 58 & 68 \\
\hline Midwest & 56 & 49 & 35 & 34 & 34 & 31 & 101 & 100 & 90 & 84 & 80 & 79 \\
\hline South & 139 & 133 & 148 & 115 & 117 & 128 & 316 & 299 & 293 & 265 & 276 & 253 \\
\hline West & 46 & 42 & 51 & 47 & 38 & 37 & 76 & 90 & 86 & 71 & 76 & 79 \\
\hline Total & 320 & 289 & 297 & 260 & 238 & 238 & 587 & 588 & 555 & 491 & 490 & 479 \\
\hline
\end{tabular}


Table 10a. Stage 3 (AIDS) attributed to heterosexual contact, by selected characteristics, 2011-2016-United States (cont)

\begin{tabular}{|c|c|c|c|c|c|c|c|c|c|c|c|c|}
\hline & \multicolumn{6}{|c|}{ Male } & \multicolumn{6}{|c|}{ Female } \\
\hline & 2011 & 2012 & 2013 & 2014 & 2015 & 2016 & 2011 & 2012 & 2013 & 2014 & 2015 & 2016 \\
\hline & No. & No. & No. & No. & No. & No. & No. & No. & No. & No. & No. & No. \\
\hline & \multicolumn{12}{|c|}{ Multiple races } \\
\hline \multicolumn{13}{|c|}{ Age at diagnosis (yr) } \\
\hline $13-14$ & 0 & 0 & 0 & 0 & 0 & 0 & 0 & 0 & 0 & 0 & 0 & 0 \\
\hline $15-19$ & 0 & 1 & 0 & 0 & 0 & 0 & 2 & 4 & 5 & 1 & 0 & 0 \\
\hline $20-24$ & 3 & 3 & 4 & 1 & 2 & 2 & 15 & 13 & 28 & 9 & 8 & 6 \\
\hline $25-29$ & 2 & 8 & 4 & 3 & 6 & 2 & 26 & 25 & 16 & 13 & 14 & 13 \\
\hline $30-34$ & 2 & 7 & 10 & 8 & 7 & 2 & 28 & 38 & 19 & 19 & 15 & 16 \\
\hline $35-39$ & 14 & 15 & 8 & 8 & 4 & 5 & 31 & 30 & 34 & 32 & 22 & 15 \\
\hline $40-44$ & 14 & 13 & 9 & 13 & 6 & 4 & 34 & 28 & 33 & 26 & 21 & 16 \\
\hline $45-49$ & 19 & 12 & 16 & 17 & 12 & 9 & 24 & 30 & 27 & 22 & 20 & 23 \\
\hline $50-54$ & 17 & 12 & 14 & 6 & 11 & 8 & 22 & 13 & 22 & 18 & 12 & 16 \\
\hline $55-59$ & 14 & 9 & 11 & 12 & 7 & 3 & 21 & 12 & 22 & 6 & 9 & 7 \\
\hline $60-64$ & 2 & 5 & 5 & 2 & 2 & 5 & 9 & 6 & 12 & 11 & 6 & 7 \\
\hline$\geq 65$ & 5 & 2 & 9 & 5 & 9 & 4 & 9 & 7 & 8 & 8 & 4 & 5 \\
\hline \multicolumn{13}{|c|}{ Region of residence ${ }^{a}$} \\
\hline Northeast & 41 & 28 & 29 & 24 & 24 & 8 & 75 & 51 & 59 & 44 & 34 & 30 \\
\hline Midwest & 6 & 13 & 8 & 11 & 4 & 6 & 24 & 26 & 15 & 21 & 13 & 15 \\
\hline South & 41 & 44 & 50 & 36 & 32 & 25 & 103 & 107 & 136 & 89 & 72 & 64 \\
\hline West & 4 & 2 & 4 & 6 & 5 & 4 & 17 & 20 & 17 & 10 & 11 & 13 \\
\hline \multirow[t]{2}{*}{ Total } & 92 & 87 & 91 & 77 & 65 & 43 & 219 & 204 & 227 & 164 & 130 & 122 \\
\hline & \multicolumn{12}{|c|}{ Total } \\
\hline \multicolumn{13}{|c|}{ Age at diagnosis (yr) } \\
\hline $13-14$ & 1 & 0 & 0 & 1 & 0 & 0 & 1 & 1 & 0 & 2 & 2 & 1 \\
\hline $15-19$ & 5 & 9 & 9 & 3 & 3 & 6 & 69 & 53 & 54 & 28 & 21 & 23 \\
\hline $20-24$ & 56 & 67 & 72 & 45 & 43 & 58 & 268 & 218 & 242 & 151 & 155 & 161 \\
\hline $25-29$ & 149 & 138 & 167 & 134 & 117 & 131 & 491 & 443 & 423 & 347 & 352 & 312 \\
\hline 30-34 & 252 & 255 & 272 & 198 & 203 & 188 & 728 & 698 & 569 & 500 & 468 & 413 \\
\hline 35-39 & 349 & 310 & 290 & 250 & 204 & 224 & 756 & 719 & 641 & 584 & 527 & 538 \\
\hline 40-44 & 436 & 416 & 358 & 302 & 283 & 258 & 760 & 752 & 673 & 565 & 504 & 479 \\
\hline $45-49$ & 535 & 477 & 398 & 346 & 312 & 289 & 752 & 701 & 681 & 523 & 512 & 430 \\
\hline 50-54 & 401 & 412 & 365 & 334 & 286 & 294 & 515 & 539 & 538 & 441 & 431 & 405 \\
\hline 55-59 & 276 & 255 & 268 & 244 & 250 & 223 & 343 & 382 & 372 & 335 & 302 & 319 \\
\hline 60-64 & 176 & 164 & 174 & 140 & 138 & 156 & 211 & 226 & 221 & 189 & 187 & 191 \\
\hline$\geq 65$ & 186 & 162 & 178 & 161 & 150 & 164 & 154 & 150 & 146 & 154 & 164 & 162 \\
\hline \multicolumn{13}{|c|}{ Region of residence ${ }^{a}$} \\
\hline Northeast & 653 & 599 & 548 & 485 & 430 & 373 & 1,085 & 1,007 & 911 & 746 & 655 & 634 \\
\hline Midwest & 274 & 261 & 234 & 184 & 187 & 199 & 539 & 503 & 439 & 437 & 362 & 396 \\
\hline South & 1,703 & 1,635 & 1,559 & 1,315 & 1,208 & 1,259 & 3,024 & 2,960 & 2,855 & 2,309 & 2,273 & 2,066 \\
\hline West & 192 & 171 & 209 & 174 & 163 & 161 & 401 & 412 & 355 & 328 & 336 & 338 \\
\hline Total & 2,822 & 2,666 & 2,550 & 2,157 & 1,989 & 1,992 & 5,049 & 4,882 & 4,560 & 3,820 & 3,625 & 3,434 \\
\hline
\end{tabular}

Note. Data for the year 2016 are preliminary (subject to change) because they are based on only a 6-month reporting delay. Data for the year 2016 should not be used when assessing trends. Numbers less than 12 , and trends based on these numbers, should be interpreted with caution. Data have been statistically adjusted to account for missing transmission category; therefore, values may not sum to column total. Data include persons with HIV infection attributed to heterosexual contact with a person known to have, or to be at high risk for, HIV infection.





${ }^{\mathrm{C}}$ Hispanics/Latinos can be of any race. 
Table 10b. Stage 3 (AIDS) attributed to heterosexual contact, by selected characteristics, 2011-2016-United States and 6 dependent areas

\begin{tabular}{|c|c|c|c|c|c|c|c|c|c|c|c|}
\hline \multicolumn{6}{|c|}{ Male } & \multicolumn{6}{|c|}{ Female } \\
\hline 2011 & 2012 & 2013 & 2014 & 2015 & 2016 & 2011 & 2012 & 2013 & 2014 & 2015 & 2016 \\
\hline No. & No. & No. & No. & No. & No. & No. & No. & No. & No. & No. & No. \\
\hline
\end{tabular}

American Indian/Alaska Native

Age at diagnosis (yr)

$13-14$
$15-19$
$20-24$
$25-29$
$30-34$
$35-39$
$40-44$
$45-49$
$50-54$
$55-59$
$60-64$
$\geq 65$

$\begin{array}{llll}0 & 0 & 0 & 0 \\ 1 & 0 & 0 & 0 \\ 1 & 0 & 0 & 0 \\ 0 & 0 & 1 & 0 \\ 1 & 1 & 2 & 1 \\ 0 & 0 & 3 & 0 \\ 1 & 0 & 0 & 1 \\ 0 & 1 & 3 & 0 \\ 1 & 0 & 0 & 0 \\ 0 & 2 & 0 & 1 \\ 1 & 1 & 1 & 0 \\ 1 & 0 & 0 & 0\end{array}$

$\begin{array}{ll}0 & 0 \\ 0 & 0 \\ 0 & 0 \\ 1 & 0 \\ 2 & 1 \\ 0 & 1 \\ 0 & 1 \\ 1 & 1 \\ 1 & 0 \\ 0 & 0 \\ 0 & 0 \\ 0 & 1\end{array}$

$\begin{array}{ll}0 & 0 \\ 1 & 0 \\ 0 & 1 \\ 2 & 0 \\ 4 & 3 \\ 3 & 2 \\ 2 & 1 \\ 0 & 2 \\ 3 & 0 \\ 1 & 2 \\ 0 & 2 \\ 0 & 1\end{array}$

$\begin{array}{llll}0 & 0 & 0 & 0 \\ 0 & 0 & 0 & 0 \\ 0 & 0 & 2 & 0 \\ 2 & 1 & 6 & 1 \\ 3 & 2 & 3 & 2 \\ 0 & 1 & 4 & 5 \\ 1 & 2 & 3 & 4 \\ 1 & 0 & 3 & 1 \\ 4 & 4 & 4 & 1 \\ 2 & 1 & 4 & 2 \\ 0 & 2 & 1 & 0 \\ 3 & 0 & 0 & 0\end{array}$

Region of residence ${ }^{\mathrm{a}}$ Northeast

Midwest

South

West

U.S. dependent areas

Total

01

$\begin{array}{ll}1 & 1\end{array}$

0

0

\begin{abstract}
0
\end{abstract}

\begin{abstract}
1
\end{abstract}
0

\begin{tabular}{rrr}
1 & 0 & 1 \\
2 & 6 & 6 \\
2 & 17 & 3 \\
8 & 5 & 6 \\
0 & 0 & 0 \\
13 & 28 & 16 \\
\hline
\end{tabular}

Asian $^{b}$

Age at diagnosis (yr)

$13-14$
$15-19$
$20-24$
$25-29$
$30-34$
$35-39$
$40-44$
$45-49$
$50-54$
$55-59$
$60-64$
$\geq 65$

Region of residence ${ }^{a}$

\begin{tabular}{|c|c|c|c|c|c|c|c|c|c|c|c|c|}
\hline Northeast & 6 & 12 & 6 & 3 & 5 & 5 & 10 & 11 & 11 & 7 & 6 & 16 \\
\hline Midwest & 8 & 7 & 2 & 8 & 6 & 5 & 7 & 8 & 9 & 7 & 9 & 8 \\
\hline South & 6 & 6 & 8 & 10 & 4 & 10 & 16 & 11 & 11 & 17 & 14 & 13 \\
\hline West & 14 & 7 & 13 & 10 & 5 & 5 & 24 & 24 & 16 & 24 & 17 & 19 \\
\hline U.S. dependent areas & 0 & 0 & 0 & 0 & 0 & 0 & 1 & 0 & 0 & 0 & 0 & 0 \\
\hline Total & 34 & 32 & 29 & 31 & 20 & 25 & 58 & 54 & 47 & 55 & 46 & 56 \\
\hline
\end{tabular}


Table 10b. Stage 3 (AIDS) attributed to heterosexual contact, by selected characteristics, 2011-2016-United States and 6 dependent areas (cont)

\begin{tabular}{|c|c|c|c|c|c|c|c|c|c|c|c|c|}
\hline & \multicolumn{6}{|c|}{ Male } & \multicolumn{6}{|c|}{ Female } \\
\hline & 2011 & 2012 & 2013 & 2014 & 2015 & 2016 & 2011 & 2012 & 2013 & 2014 & 2015 & 2016 \\
\hline & No. & No. & No. & No. & No. & No. & No. & No. & No. & No. & No. & No. \\
\hline & \multicolumn{12}{|c|}{ Black/African American } \\
\hline \multicolumn{13}{|l|}{ Age at diagnosis (yr) } \\
\hline $13-14$ & 1 & 0 & 0 & 1 & 0 & 0 & 1 & 1 & 0 & 1 & 2 & 0 \\
\hline $15-19$ & 4 & 7 & 8 & 3 & 3 & 6 & 54 & 41 & 37 & 19 & 20 & 16 \\
\hline $20-24$ & 37 & 47 & 48 & 33 & 30 & 37 & 197 & 154 & 157 & 106 & 107 & 105 \\
\hline $25-29$ & 83 & 89 & 102 & 75 & 65 & 86 & 318 & 306 & 302 & 239 & 241 & 207 \\
\hline $30-34$ & 164 & 150 & 152 & 122 & 113 & 116 & 493 & 480 & 391 & 337 & 325 & 269 \\
\hline $35-39$ & 215 & 198 & 168 & 155 & 129 & 141 & 507 & 500 & 423 & 372 & 349 & 349 \\
\hline 40-44 & 294 & 280 & 229 & 183 & 191 & 169 & 493 & 508 & 451 & 373 & 335 & 321 \\
\hline $45-49$ & 376 & 334 & 260 & 219 & 196 & 180 & 511 & 468 & 466 & 352 & 336 & 276 \\
\hline 50-54 & 278 & 277 & 237 & 226 & 179 & 187 & 352 & 372 & 344 & 295 & 263 & 250 \\
\hline $55-59$ & 188 & 168 & 175 & 160 & 176 & 156 & 229 & 249 & 251 & 211 & 189 & 189 \\
\hline $60-64$ & 114 & 108 & 121 & 90 & 90 & 106 & 138 & 148 & 129 & 133 & 121 & 127 \\
\hline$\geq 65$ & 115 & 115 & 109 & 107 & 92 & 111 & 103 & 95 & 88 & 105 & 114 & 93 \\
\hline \multicolumn{13}{|l|}{ Region of residence ${ }^{a}$} \\
\hline Northeast & 362 & 324 & 296 & 262 & 244 & 217 & 603 & 576 & 500 & 430 & 386 & 307 \\
\hline Midwest & 164 & 159 & 157 & 112 & 112 & 129 & 362 & 325 & 287 & 291 & 227 & 257 \\
\hline South & 1,277 & 1,233 & 1,097 & 950 & 850 & 891 & 2,275 & 2,264 & 2,131 & 1,707 & 1,660 & 1,520 \\
\hline West & 63 & 53 & 57 & 44 & 54 & 56 & 155 & 156 & 119 & 113 & 125 & 119 \\
\hline U.S. dependent areas & 3 & 3 & 2 & 3 & 2 & 1 & 1 & 2 & 3 & 2 & 3 & 1 \\
\hline Total & 1,869 & 1,772 & 1,609 & 1,371 & 1,262 & 1,294 & 3,396 & 3,323 & 3,040 & 2,543 & 2,401 & 2,204 \\
\hline & \multicolumn{12}{|c|}{ Hispanic/Latino ${ }^{C}$} \\
\hline \multicolumn{13}{|l|}{ Age at diagnosis (yr) } \\
\hline $13-14$ & 0 & 0 & 0 & 0 & 0 & 0 & 1 & 0 & 0 & 1 & 0 & 1 \\
\hline $15-19$ & 0 & 1 & 0 & 0 & 0 & 0 & 11 & 2 & 10 & 9 & 2 & 5 \\
\hline $20-24$ & 10 & 12 & 17 & 8 & 12 & 16 & 37 & 36 & 37 & 16 & 23 & 29 \\
\hline $25-29$ & 56 & 29 & 43 & 44 & 27 & 32 & 94 & 73 & 60 & 48 & 50 & 52 \\
\hline $30-34$ & 76 & 76 & 91 & 50 & 66 & 49 & 118 & 102 & 101 & 92 & 71 & 70 \\
\hline $35-39$ & 90 & 82 & 83 & 72 & 53 & 60 & 121 & 109 & 115 & 106 & 91 & 89 \\
\hline $40-44$ & 91 & 84 & 79 & 75 & 65 & 55 & 145 & 120 & 116 & 102 & 87 & 75 \\
\hline $45-49$ & 92 & 92 & 82 & 77 & 69 & 78 & 145 & 116 & 109 & 83 & 83 & 71 \\
\hline $50-54$ & 74 & 70 & 79 & 55 & 67 & 54 & 94 & 96 & 104 & 63 & 75 & 76 \\
\hline $55-59$ & 47 & 51 & 56 & 46 & 34 & 40 & 49 & 66 & 50 & 60 & 42 & 62 \\
\hline $60-64$ & 29 & 28 & 31 & 32 & 21 & 23 & 34 & 48 & 37 & 28 & 29 & 26 \\
\hline$\geq 65$ & 39 & 33 & 38 & 30 & 28 & 28 & 38 & 43 & 34 & 21 & 24 & 38 \\
\hline \multicolumn{13}{|l|}{ Region of residence ${ }^{a}$} \\
\hline Northeast & 165 & 169 & 152 & 131 & 107 & 101 & 304 & 269 & 255 & 194 & 170 & 212 \\
\hline Midwest & 40 & 32 & 27 & 19 & 30 & 25 & 41 & 42 & 36 & 32 & 26 & 32 \\
\hline South & 238 & 219 & 255 & 202 & 205 & 202 & 310 & 278 & 276 & 229 & 233 & 213 \\
\hline West & 60 & 63 & 80 & 64 & 54 & 55 & 117 & 111 & 110 & 100 & 100 & 99 \\
\hline U.S. dependent areas & 103 & 74 & 86 & 71 & 46 & 52 & 115 & 111 & 96 & 74 & 47 & 41 \\
\hline Total & 606 & 557 & 600 & 487 & 442 & 435 & 887 & 811 & 773 & 629 & 576 & 597 \\
\hline
\end{tabular}


Table 10b. Stage 3 (AIDS) attributed to heterosexual contact, by selected characteristics, 2011-2016-United States and 6 dependent areas (cont)

\begin{tabular}{|c|c|c|c|c|c|c|c|c|c|c|c|}
\hline \multicolumn{6}{|c|}{ Male } & \multicolumn{6}{|c|}{ Female } \\
\hline 2011 & 2012 & 2013 & 2014 & 2015 & 2016 & 2011 & 2012 & 2013 & 2014 & 2015 & 2016 \\
\hline No. & No. & No. & No. & No. & No. & No. & No. & No. & No. & No. & No. \\
\hline
\end{tabular}

Age at diagnosis (yr)

\begin{tabular}{|c|c|c|c|c|c|c|c|c|c|c|c|c|}
\hline $13-14$ & 0 & 0 & 0 & 0 & 0 & 0 & 0 & 0 & 0 & 0 & 0 & 0 \\
\hline $15-19$ & 0 & 0 & 0 & 0 & 0 & 0 & 0 & 0 & 0 & 0 & 0 & 0 \\
\hline 20-24 & 0 & 0 & 0 & 0 & 0 & 0 & 0 & 0 & 1 & 0 & 0 & 0 \\
\hline $25-29$ & 0 & 0 & 0 & 0 & 0 & 0 & 1 & 0 & 0 & 0 & 0 & 0 \\
\hline $30-34$ & 0 & 0 & 0 & 0 & 0 & 0 & 0 & 1 & 0 & 1 & 1 & 1 \\
\hline $35-39$ & 1 & 0 & 0 & 0 & 1 & 1 & 1 & 0 & 0 & 0 & 1 & 1 \\
\hline $40-44$ & 0 & 0 & 0 & 2 & 0 & 0 & 0 & 0 & 0 & 0 & 0 & 1 \\
\hline $45-49$ & 0 & 0 & 0 & 0 & 1 & 1 & 1 & 1 & 2 & 0 & 0 & 0 \\
\hline $50-54$ & 0 & 0 & 0 & 0 & 2 & 0 & 0 & 1 & 2 & 0 & 0 & 1 \\
\hline $55-59$ & 0 & 0 & 0 & 0 & 0 & 0 & 1 & 0 & 0 & 1 & 0 & 0 \\
\hline $60-64$ & 0 & 0 & 0 & 0 & 0 & 0 & 0 & 0 & 0 & 1 & 0 & 0 \\
\hline$\geq 65$ & 0 & 0 & 1 & 0 & 0 & 0 & 0 & 0 & 0 & 0 & 0 & 0 \\
\hline
\end{tabular}

Region of residence ${ }^{a}$

Northeas

Midwest

South

West

U.S. dependent areas

Total

0
0
0
1
0
1

$\begin{array}{lll}0 & 0 & 0 \\ 0 & 0 & 0 \\ 0 & 0 & 1 \\ 1 & 1 & 1 \\ 0 & 0 & 0 \\ 1 & 1 & 2\end{array}$

0
0
0
3
0
3

\begin{tabular}{lllllll}
0 & 0 & 0 & 0 & 0 & 0 & 0 \\
1 & 0 & 0 & 0 & 0 & 1 & 0 \\
1 & 0 & 0 & 3 & 1 & 0 & 0 \\
0 & 4 & 3 & 2 & 2 & 1 & 3 \\
0 & 0 & 0 & 0 & 0 & 0 & 1 \\
$\mathbf{2}$ & $\mathbf{4}$ & $\mathbf{3}$ & $\mathbf{5}$ & $\mathbf{3}$ & $\mathbf{2}$ & $\mathbf{4}$ \\
\hline
\end{tabular}

White

Age at diagnosis (yr)




Table 10b. Stage 3 (AIDS) attributed to heterosexual contact, by selected characteristics, 2011-2016-United States and 6 dependent areas (cont)

\begin{tabular}{|c|c|c|c|c|c|c|c|c|c|c|c|c|}
\hline & \multicolumn{6}{|c|}{ Male } & \multicolumn{6}{|c|}{ Female } \\
\hline & 2011 & 2012 & 2013 & 2014 & 2015 & 2016 & 2011 & 2012 & 2013 & 2014 & 2015 & 2016 \\
\hline & No. & No. & No. & No. & No. & No. & No. & No. & No. & No. & No. & No. \\
\hline & \multicolumn{12}{|c|}{ Multiple races } \\
\hline \multicolumn{13}{|l|}{ Age at diagnosis (yr) } \\
\hline $13-14$ & 0 & 0 & 0 & 0 & 0 & 0 & 0 & 0 & 0 & 0 & 0 & 0 \\
\hline $15-19$ & 0 & 1 & 0 & 0 & 0 & 0 & 2 & 4 & 5 & 1 & 0 & 0 \\
\hline $20-24$ & 3 & 3 & 4 & 1 & 2 & 2 & 15 & 13 & 28 & 9 & 8 & 6 \\
\hline $25-29$ & 2 & 8 & 4 & 3 & 6 & 2 & 26 & 25 & 16 & 13 & 14 & 13 \\
\hline $30-34$ & 2 & 7 & 10 & 8 & 7 & 2 & 28 & 38 & 19 & 19 & 15 & 16 \\
\hline $35-39$ & 14 & 15 & 8 & 8 & 4 & 5 & 33 & 30 & 34 & 32 & 22 & 15 \\
\hline $40-44$ & 14 & 13 & 9 & 13 & 6 & 4 & 34 & 28 & 33 & 26 & 21 & 16 \\
\hline $45-49$ & 19 & 12 & 16 & 17 & 12 & 9 & 24 & 30 & 27 & 22 & 20 & 23 \\
\hline $50-54$ & 17 & 12 & 14 & 6 & 11 & 8 & 22 & 13 & 22 & 18 & 12 & 16 \\
\hline $55-59$ & 14 & 9 & 11 & 12 & 7 & 3 & 21 & 12 & 22 & 6 & 9 & 7 \\
\hline $60-64$ & 2 & 5 & 5 & 2 & 2 & 5 & 9 & 6 & 12 & 11 & 6 & 7 \\
\hline$\geq 65$ & 5 & 2 & 9 & 5 & 9 & 4 & 9 & 7 & 8 & 8 & 4 & 5 \\
\hline \multicolumn{13}{|l|}{ Region of residence $^{a}$} \\
\hline Northeast & 41 & 28 & 29 & 24 & 24 & 8 & 75 & 51 & 59 & 44 & 34 & 30 \\
\hline Midwest & 6 & 13 & 8 & 11 & 4 & 6 & 24 & 26 & 15 & 21 & 13 & 15 \\
\hline South & 41 & 44 & 50 & 36 & 32 & 25 & 103 & 107 & 136 & 89 & 72 & 64 \\
\hline West & 4 & 2 & 4 & 6 & 5 & 4 & 17 & 20 & 17 & 10 & 11 & 13 \\
\hline U.S. dependent areas & 0 & 0 & 0 & 0 & 0 & 0 & 2 & 0 & 0 & 0 & 0 & 0 \\
\hline \multirow[t]{2}{*}{ Total } & 92 & 87 & 91 & 77 & 65 & 43 & 221 & 204 & 227 & 164 & 130 & 122 \\
\hline & \multicolumn{12}{|c|}{ Total } \\
\hline \multicolumn{13}{|l|}{ Age at diagnosis (yr) } \\
\hline $13-14$ & 1 & 0 & 0 & 1 & 0 & 0 & 1 & 1 & 0 & 2 & 2 & 1 \\
\hline $15-19$ & 5 & 9 & 9 & 3 & 3 & 6 & 71 & 53 & 56 & 30 & 21 & 23 \\
\hline $20-24$ & 57 & 68 & 73 & 45 & 45 & 58 & 270 & 221 & 243 & 152 & 156 & 163 \\
\hline $25-29$ & 154 & 140 & 168 & 138 & 118 & 134 & 499 & 449 & 425 & 349 & 353 & 314 \\
\hline 30-34 & 257 & 259 & 277 & 200 & 205 & 191 & 739 & 710 & 577 & 513 & 474 & 413 \\
\hline $35-39$ & 360 & 320 & 296 & 258 & 209 & 229 & 770 & 730 & 654 & 593 & 532 & 546 \\
\hline $40-44$ & 455 & 428 & 368 & 314 & 290 & 263 & 774 & 769 & 689 & 581 & 512 & 484 \\
\hline $45-49$ & 555 & 492 & 410 & 359 & 321 & 302 & 773 & 718 & 699 & 529 & 522 & 439 \\
\hline $50-54$ & 419 & 424 & 380 & 344 & 299 & 301 & 540 & 556 & 553 & 451 & 438 & 409 \\
\hline $55-59$ & 283 & 262 & 284 & 253 & 252 & 230 & 350 & 392 & 386 & 345 & 307 & 327 \\
\hline $60-64$ & 183 & 170 & 185 & 150 & 140 & 159 & 217 & 236 & 225 & 192 & 187 & 195 \\
\hline$\geq 65$ & 199 & 171 & 188 & 168 & 157 & 173 & 163 & 160 & 152 & 158 & 171 & 162 \\
\hline \multicolumn{13}{|l|}{ Region of residence ${ }^{a}$} \\
\hline Northeast & 653 & 599 & 548 & 485 & 430 & 373 & 1,085 & 1,007 & 911 & 746 & 655 & 634 \\
\hline Midwest & 274 & 261 & 234 & 184 & 187 & 199 & 539 & 503 & 439 & 437 & 362 & 396 \\
\hline South & 1,703 & 1,635 & 1,559 & 1,315 & 1,208 & 1,259 & 3,024 & 2,960 & 2,855 & 2,309 & 2,273 & 2,066 \\
\hline West & 192 & 171 & 209 & 174 & 163 & 161 & 401 & 412 & 355 & 328 & 336 & 338 \\
\hline U.S. dependent areas & 106 & 77 & 88 & 75 & 49 & 53 & 119 & 113 & 99 & 76 & 50 & 44 \\
\hline Total & 2,928 & 2,743 & 2,638 & 2,232 & 2,038 & 2,045 & 5,168 & 4,994 & 4,659 & 3,896 & 3,675 & 3,478 \\
\hline
\end{tabular}

Note. Data for the year 2016 are preliminary (subject to change) because they are based on only a 6-month reporting delay. Data for the year 2016 should not be used when assessing trends. Numbers less than 12 , and trends based on these numbers, should be interpreted with caution. Data have been statistically adjusted to account for missing transmission category; therefore, values may not sum to column total. Data include persons with HIV infection attributed to heterosexual contact with a person known to have, or to be at high risk for, HIV infection.

a Data are based on residence at time of stage 3 (AIDS) classification.

${ }^{b}$ Includes Asian/Pacific Islander legacy cases (see Technical Notes).

$\mathrm{C}_{\text {Hispanics/Latinos can be of any race. }}$ 


\begin{tabular}{|c|c|c|c|c|c|c|c|c|c|c|c|c|}
\hline \multirow[b]{2}{*}{ Race/ethnicity } & \multicolumn{2}{|c|}{2011} & \multicolumn{2}{|c|}{2012} & \multicolumn{2}{|c|}{2013} & \multicolumn{2}{|c|}{2014} & \multicolumn{2}{|c|}{2015} & \multicolumn{2}{|c|}{2016} \\
\hline & No. & Rate $^{a}$ & No. & Rate $^{a}$ & No. & Rate $^{a}$ & No. & Rate $^{a}$ & No. & Rate $^{a}$ & No. & Rate $^{\mathrm{a}}$ \\
\hline American Indian/Alaska Native & 0 & 0.0 & 0 & 0.0 & 0 & 0.0 & 3 & 0.7 & 2 & 0.4 & 0 & 0.0 \\
\hline Asian & 8 & 0.3 & 13 & 0.5 & 16 & 0.6 & 12 & 0.5 & 9 & 0.4 & 8 & 0.3 \\
\hline Black/African American & 118 & 1.6 & 168 & 2.3 & 122 & 1.7 & 112 & 1.6 & 87 & 1.2 & 78 & 1.1 \\
\hline Hispanic/Latino ${ }^{b}$ & 24 & 0.2 & 16 & 0.1 & 11 & 0.1 & 18 & 0.1 & 13 & 0.1 & 16 & 0.1 \\
\hline Native Hawaiian/Other Pacific Islander & 0 & 0.0 & 0 & 0.0 & 0 & 0.0 & 1 & 1.0 & 0 & 0.0 & 0 & 0.0 \\
\hline White & 35 & 0.1 & 32 & 0.1 & 24 & 0.1 & 29 & 0.1 & 18 & 0.1 & 16 & 0.1 \\
\hline Multiple races & 13 & 0.6 & 11 & 0.5 & 13 & 0.6 & 5 & 0.2 & 6 & 0.3 & 4 & 0.2 \\
\hline Total & 198 & 0.4 & 240 & 0.5 & 186 & 0.4 & 180 & 0.3 & 135 & 0.3 & 122 & 0.2 \\
\hline
\end{tabular}

Note. Data for the year 2016 are preliminary (subject to change) because they are based on only a 6-month reporting delay. Data for the year 2016 should not be used when assessing trends. Numbers less than 12 , and rates and trends based on these numbers, should be interpreted with caution.

a Rates are per 100,000 population.

${ }^{b}$ Hispanics/Latinos can be of any race.

Table 11b.Diagnoses of HIV infection among children aged <13 years, by race/ethnicity, 2011-2016—United States and 6 dependent areas

\begin{tabular}{|c|c|c|c|c|c|c|}
\hline Race/ethnicity & $\begin{array}{c}2011 \\
\text { No. }\end{array}$ & $\begin{array}{c}2012 \\
\text { No. }\end{array}$ & $\begin{array}{c}2013 \\
\text { No. }\end{array}$ & $\begin{array}{c}2014 \\
\text { No. }\end{array}$ & $\begin{array}{c}2015 \\
\text { No. }\end{array}$ & $\begin{array}{c}2016 \\
\text { No. }\end{array}$ \\
\hline American Indian/Alaska Native & 0 & 0 & 0 & 3 & 2 & 0 \\
\hline Black/African American & 118 & 168 & 122 & 112 & 87 & 78 \\
\hline Hispanic/Latino ${ }^{a}$ & 26 & 16 & 11 & 18 & 13 & 16 \\
\hline White & 35 & 32 & 24 & 29 & 18 & 16 \\
\hline Multiple races & 13 & 11 & 13 & 5 & 6 & 4 \\
\hline Total & 200 & 240 & 186 & 180 & 135 & 122 \\
\hline
\end{tabular}

Note. Data for the year 2016 are preliminary (subject to change) because they are based on only a 6-month reporting delay. Data for the year 2016

should not be used when assessing trends. Numbers less than 12 , and trends based on these numbers, should be interpreted with caution.

${ }^{a}$ Hispanics/Latinos can be of any race. 
Table 12a.Stage 3 (AIDS) among children aged <13 years, by race/ethnicity, 2011-2016 and cumulative—United States

2011

2012

2013

2014

2015

2016

Cumulative $^{\mathrm{a}}$

Race/ethnicity

No. Rate

$\begin{array}{rc}\text { No. } & \text { Rate } \\ 0 & 0.0 \\ 0 & 0.0 \\ 9 & 0.1 \\ 1 & 0.0 \\ 0 & 0.0 \\ 1 & 0.0 \\ 0 & 0.0 \\ 11 & \mathbf{0 . 0}\end{array}$

No.

Rate $^{b}$

No. Rate $^{\mathrm{b}}$

No. Rate ${ }^{b}$

No.

No.

American Indian/Alaska Native

Asian $^{\mathrm{C}}$

Black/African American

Hispanic/Latino $^{d}$

Native Hawaiian/Other Pacific Islander

White

Multiple races

$\quad 0.0$

$\begin{array}{llrll}0 & 0.0 & 1 & 0.2 & \\ 0 & 0.0 & 0 & 0.0 & \\ 5 & 0.1 & 49 & 0.7 & 28 \\ 2 & 0.0 & 13 & 0.1 & \\ 0 & 0.0 & 0 & 0.0 & \\ 0 & 0.0 & 6 & 0.0 \\ 2 & 0.1 & 2 & 0.1 & \\ \mathbf{9} & \mathbf{0 . 0} & \mathbf{7 1} & \mathbf{0 . 1} & \mathbf{4 1}\end{array}$

Note. Data for the year 2016 are preliminary (subject to change) because they are based on only a 6-month reporting delay. Data for the year 2016 should not be used when assessing trends.


(see Technical Notes)

a From the beginning of the epidemic through 2016.

${ }^{b}$ Rates are per 100,000 population.



$\mathrm{d}_{\text {Hispanics/Latinos can be of any race. }}$

${ }^{\mathrm{e}}$ Cumulative total includes 2 persons whose race/ethnicity is unknown.

Table 12b.Stage 3 (AIDS) among children aged <13 years, by race/ethnicity, 2011-2016 and cumulative-United States and 6 dependent areas

\begin{tabular}{|c|c|c|c|c|c|c|c|}
\hline Race/ethnicity & $\begin{array}{c}2011 \\
\text { No. }\end{array}$ & $\begin{array}{c}2012 \\
\text { No. }\end{array}$ & $\begin{array}{c}2013 \\
\text { No. }\end{array}$ & $\begin{array}{c}2014 \\
\text { No. }\end{array}$ & $\begin{array}{c}2015 \\
\text { No. }\end{array}$ & $\begin{array}{c}2016 \\
\text { No. }\end{array}$ & $\begin{array}{c}\text { Cumulative }^{a} \\
\text { No. }\end{array}$ \\
\hline American Indian/Alaska Native & 0 & 0 & 0 & 1 & 0 & 1 & 28 \\
\hline Asian $^{\mathrm{b}}$ & 2 & 0 & 0 & 0 & 2 & 1 & 51 \\
\hline Black/African American & 12 & 9 & 5 & 49 & 28 & 26 & 5,646 \\
\hline Hispanic/Latino ${ }^{c}$ & 2 & 1 & 2 & 13 & 5 & 2 & 2,449 \\
\hline Native Hawaiian/Other Pacific Islander & 1 & 0 & 0 & 0 & 0 & 0 & 7 \\
\hline White & 2 & 1 & 0 & 6 & 3 & 5 & 1,536 \\
\hline Multiple races & 0 & 0 & 2 & 2 & 2 & 4 & 281 \\
\hline Total $^{d}$ & 19 & 11 & 9 & 71 & 40 & 39 & 10,000 \\
\hline
\end{tabular}

Note. Data for the year 2016 are preliminary (subject to change) because they are based on only a 6-month reporting delay. Data for the year 2016 should not be used when assessing trends. Numbers less than 12, and trends based on these numbers, should be interpreted with caution. The criteria for stage 3 (AIDS) classification among pediatric cases were trends. Numbers less than 12, and trends 2014 (see Technical Notes).




Table 13. Stage 3 (AIDS) among children aged $<13$ years, by year of diagnosis, 1992-2016-United States and 6 dependent areas

\begin{tabular}{|c|c|c|}
\hline Year of diagnosis & $\begin{array}{c}\text { United States } \\
\text { No. }\end{array}$ & $\begin{array}{c}\text { United States and } \\
\text { dependent areas } \\
\text { No. }\end{array}$ \\
\hline 1992 & 906 & 955 \\
\hline 1993 & 885 & 923 \\
\hline 1994 & 818 & 844 \\
\hline 1995 & 675 & 697 \\
\hline 1996 & 521 & 535 \\
\hline 1997 & 336 & 348 \\
\hline 1998 & 250 & 257 \\
\hline 1999 & 201 & 206 \\
\hline 2000 & 135 & 137 \\
\hline 2001 & 130 & 131 \\
\hline 2002 & 108 & 115 \\
\hline 2003 & 75 & 77 \\
\hline 2004 & 61 & 62 \\
\hline 2005 & 55 & 55 \\
\hline 2006 & 40 & 43 \\
\hline 2007 & 35 & 36 \\
\hline 2008 & 39 & 40 \\
\hline 2009 & 15 & 16 \\
\hline 2010 & 22 & 22 \\
\hline 2011 & 18 & 19 \\
\hline 2012 & 11 & 11 \\
\hline 2013 & 9 & 9 \\
\hline 2014 & 71 & 71 \\
\hline 2015 & 40 & 40 \\
\hline 2016 & 38 & 39 \\
\hline
\end{tabular}

Note. Data for the year 2016 are preliminary (subject to change) because they are based on only a 6 -month reporting delay. Data for the year 2016 should not be used when assessing trends.

Numbers less than 12, and trends based on these numbers, should be interpreted with caution.

The criteria for stage 3 (AIDS) classification among pediatric cases were expanded in 2014 (see

Technical Notes). 
Table 14. Diagnoses of HIV infection among adult and adolescent Hispanics/Latinos, by transmission category and place of birth, 2016-United States and 6 dependent areas

\begin{tabular}{|c|c|c|c|c|c|c|c|c|c|c|c|c|c|c|}
\hline \multirow[b]{2}{*}{ Transmission category $^{a}$} & \multicolumn{2}{|c|}{$\begin{array}{l}\text { Central } \\
\text { America }\end{array}$} & \multicolumn{2}{|c|}{ Cuba } & \multicolumn{2}{|c|}{ Mexico } & \multicolumn{2}{|c|}{ Puerto Rico } & \multicolumn{2}{|c|}{ South America } & \multicolumn{2}{|c|}{ United States } & \multicolumn{2}{|c|}{ Total } \\
\hline & No. & $\%$ & No. & $\%$ & No. & $\%$ & No. & $\%$ & No. & $\%$ & No. & $\%$ & No. ${ }^{b}$ & $\%$ \\
\hline \multicolumn{15}{|l|}{ Male adult or adolescent } \\
\hline Male-to-male sexual contact & 391 & 84.0 & 464 & 91.2 & 898 & 85.1 & 386 & 66.0 & 633 & 92.6 & 2,697 & 86.5 & 7,689 & 85.4 \\
\hline Injection drug use & 11 & 2.3 & 3 & 0.6 & 29 & 2.8 & 64 & 11.0 & 4 & 0.6 & 131 & 4.2 & 345 & 3.8 \\
\hline $\begin{array}{l}\text { Male-to-male sexual contact } \\
\text { and injection drug use }\end{array}$ & 7 & 1.4 & 6 & 1.3 & 25 & 2.4 & 23 & 3.9 & 6 & 0.9 & 132 & 4.2 & 278 & 3.1 \\
\hline Heterosexual contact ${ }^{c}$ & 56 & 12.0 & 35 & 6.9 & 102 & 9.7 & 112 & 19.2 & 40 & 5.8 & 154 & 4.9 & 680 & 7.6 \\
\hline Other ${ }^{d}$ & 2 & 0.3 & 0 & 0.0 & 1 & 0.1 & 0 & 0.0 & 0 & 0.0 & 3 & 0.1 & 7 & 0.1 \\
\hline Subtotal & 465 & 100 & 509 & 100 & 1,055 & 100 & 585 & 100 & 683 & 100 & 3,117 & 100 & 8,999 & 100 \\
\hline \multicolumn{15}{|l|}{ Female adult or adolescent } \\
\hline Injection drug use & 5 & 4.5 & 0 & 0.5 & 4 & 4.5 & 15 & 9.6 & 2 & 2.8 & 71 & 18 & 152 & 11.9 \\
\hline Heterosexual $\operatorname{contact}^{\mathrm{C}}$ & 98 & 95.1 & 42 & 99.3 & 91 & 95.2 & 137 & 90.4 & 56 & 97.1 & 322 & 81.6 & 1,121 & 87.8 \\
\hline Other $^{d}$ & 0 & 0.4 & 0 & 0.2 & 0 & 0.3 & 0 & 0.0 & 0 & 0.2 & 1 & 0.3 & 4 & 0.3 \\
\hline Subtotal & 103 & 100 & 42 & 100 & 96 & 100 & 152 & 100 & 58 & 100 & 394 & 100 & 1,277 & 100 \\
\hline Total & 568 & 100 & 551 & 100 & 1,151 & 100 & 737 & 100 & 741 & 100 & 3,511 & 100 & 10,276 & 100 \\
\hline
\end{tabular}


numbers, should be interpreted with caution.

a Data have been statistically adjusted to account for missing transmission category; therefore, values may not sum to column subtotals and total.

$\mathrm{b}$ Entries include persons whose place of birth is not among those listed and persons whose place of birth is unknown.

${ }^{c}$ Heterosexual contact with a person known to have, or to be at high risk for, HIV infection.




Table 15. Stage 3 (AIDS) among adult and adolescent Hispanics/Latinos, by transmission category and place of birth, 2016United States and 6 dependent areas

\begin{tabular}{|c|c|c|c|c|c|c|c|c|c|c|c|c|c|c|}
\hline \multirow[b]{2}{*}{ Transmission category ${ }^{\mathrm{a}}$} & \multicolumn{2}{|c|}{$\begin{array}{l}\text { Central } \\
\text { America }\end{array}$} & \multicolumn{2}{|c|}{ Cuba } & \multicolumn{2}{|c|}{ Mexico } & \multicolumn{2}{|c|}{ Puerto Rico } & \multicolumn{2}{|c|}{ South America } & \multicolumn{2}{|c|}{ United States } & \multicolumn{2}{|c|}{ Total } \\
\hline & No. & $\%$ & No. & $\%$ & No. & $\%$ & No. & $\%$ & No. & $\%$ & No. & $\%$ & No. ${ }^{b}$ & $\%$ \\
\hline \multicolumn{15}{|l|}{ Male adult or adolescent } \\
\hline Male-to-male sexual contact & 212 & 77.7 & 115 & 86.1 & 436 & 77.5 & 123 & 43.4 & 129 & 78.7 & 1,006 & 76.7 & 2,692 & 74.6 \\
\hline Injection drug use & 12 & 4.2 & 1 & 1.1 & 30 & 5.4 & 73 & 25.7 & 5 & 2.9 & 100 & 7.6 & 288 & 8.0 \\
\hline $\begin{array}{l}\text { Male-to-male sexual contact } \\
\text { and injection drug use }\end{array}$ & 5 & 1.6 & 1 & 1.0 & 14 & 2.6 & 24 & 8.3 & 3 & 2.0 & 98 & 7.5 & 174 & 4.8 \\
\hline Heterosexual contact ${ }^{\mathrm{c}}$ & 45 & 16.3 & 16 & 11.9 & 81 & 14.4 & 61 & 21.5 & 27 & 16.2 & 93 & 7.1 & 435 & 12.1 \\
\hline Other ${ }^{d}$ & 0 & 0.1 & 0 & 0.0 & 1 & 0.1 & 3 & 1.1 & 0 & 0.1 & 13 & 1.0 & 20 & 0.6 \\
\hline Subtotal & 273 & 100 & 133 & 100 & 563 & 100 & 284 & 100 & 164 & 100 & 1,311 & 100 & 3,609 & 100 \\
\hline \multicolumn{15}{|l|}{ Female adult or adolescent } \\
\hline Injection drug use & 4 & 5.5 & 1 & 7.9 & 4 & 6.1 & 13 & 15.3 & 4 & 13.7 & 67 & 22.6 & 121 & 16.4 \\
\hline Heterosexual contact ${ }^{c}$ & 62 & 93.8 & 13 & 91.4 & 57 & 93.6 & 74 & 84.7 & 23 & 85.9 & 209 & 71.1 & 596 & 80.7 \\
\hline Other ${ }^{d}$ & 1 & 0.8 & 0 & 0.7 & 0 & 0.3 & 0 & 0.0 & 0 & 0.4 & 19 & 6.3 & 22 & 2.9 \\
\hline Subtotal & 66 & 100 & 14 & 100 & 61 & 100 & 87 & 100 & 27 & 100 & 294 & 100 & 739 & 100 \\
\hline Total & 339 & 100 & 147 & 100 & 624 & 100 & 371 & 100 & 191 & 100 & 1,605 & 100 & 4,348 & 100 \\
\hline
\end{tabular}

Note. Data for the year 2016 are preliminary (subject to change) because they are based on only a 6 -month reporting delay. Numbers less than 12 , and percentages based on these numbers, should be interpreted with caution.

${ }^{a}$ Data have been statistically adjusted to account for missing transmission category; therefore, values may not sum to column subtotals and total.

${ }^{b}$ Entries include persons whose place of birth is not among those listed and persons whose place of birth is unknown.

${ }^{c}$ Heterosexual contact with a person known to have, or to be at high risk for, HIV infection.






\begin{tabular}{|c|c|c|c|c|c|c|c|c|c|c|}
\hline & \multicolumn{2}{|c|}{2011} & \multicolumn{2}{|c|}{2012} & \multicolumn{2}{|c|}{2013} & \multicolumn{2}{|c|}{2014} & \multicolumn{2}{|c|}{2015} \\
\hline & No. & Rate $^{a}$ & No. & Rate $^{a}$ & No. & Rate $^{a}$ & No. & Rate $^{a}$ & No. & Rate $^{a}$ \\
\hline \multicolumn{11}{|l|}{ Age at death (yr) } \\
\hline$<13$ & 1 & 0.0 & 4 & 0.0 & 1 & 0.0 & 1 & 0.0 & 4 & 0.0 \\
\hline 13-14 & 2 & 0.0 & 2 & 0.0 & 1 & 0.0 & 3 & 0.0 & 0 & 0.0 \\
\hline 15-19 & 25 & 0.1 & 23 & 0.1 & 9 & 0.0 & 13 & 0.1 & 12 & 0.1 \\
\hline $20-24$ & 203 & 0.9 & 174 & 0.8 & 170 & 0.7 & 171 & 0.7 & 143 & 0.6 \\
\hline $25-29$ & 420 & 2.0 & 417 & 1.9 & 426 & 2.0 & 366 & 1.7 & 391 & 1.7 \\
\hline 30-34 & 686 & 3.3 & 704 & 3.4 & 632 & 3.0 & 644 & 3.0 & 581 & 2.7 \\
\hline $35-39$ & 1,064 & 5.4 & 958 & 4.9 & 858 & 4.4 & 889 & 4.5 & 787 & 3.9 \\
\hline $40-44$ & 1,850 & 8.8 & 1,639 & 7.8 & 1,479 & 7.1 & 1,358 & 6.6 & 1,201 & 6.0 \\
\hline $45-49$ & 2,932 & 13.2 & 2,662 & 12.3 & 2,459 & 11.6 & 2,202 & 10.6 & 1,931 & 9.3 \\
\hline $50-54$ & 3,152 & 14.0 & 3,141 & 13.9 & 3,095 & 13.7 & 2,999 & 13.3 & 2,881 & 12.9 \\
\hline $55-59$ & 2,675 & 13.2 & 2,672 & 12.9 & 2,755 & 13.0 & 2,926 & 13.6 & 2,776 & 12.8 \\
\hline 60-64 & 1,745 & 9.8 & 1,877 & 10.5 & 1,961 & 10.8 & 2,225 & 12.0 & 2,109 & 11.1 \\
\hline$\geq 65$ & 1,842 & 4.5 & 2,011 & 4.7 & 2,257 & 5.1 & 2,457 & 5.3 & 2,642 & 5.5 \\
\hline \multicolumn{11}{|l|}{ Race/ethnicity } \\
\hline American Indian/Alaska Native & 58 & 2.5 & 54 & 2.3 & 64 & 2.7 & 61 & 2.6 & 58 & 2.4 \\
\hline Asian ${ }^{b}$ & 87 & 0.6 & 72 & 0.5 & 73 & 0.5 & 62 & 0.4 & 76 & 0.4 \\
\hline Black/African American & 7,914 & 20.6 & 7,682 & 19.8 & 7,486 & 19.1 & 7,420 & 18.8 & 6,960 & 17.5 \\
\hline Hispanic/Latino ${ }^{c}$ & 2,734 & 5.3 & 2,485 & 4.7 & 2,651 & 4.9 & 2,765 & 5.0 & 2,481 & 4.4 \\
\hline Native Hawaiian/Other Pacific Islander & 10 & 2.0 & 7 & 1.3 & 10 & 1.9 & 8 & 1.5 & 6 & 1.1 \\
\hline White & 4,991 & 2.5 & 5,122 & 2.6 & 4,909 & 2.5 & 5,017 & 2.5 & 4,915 & 2.5 \\
\hline Multiple races & 801 & 13.7 & 840 & 14.0 & 906 & 14.6 & 917 & 14.4 & 957 & 14.6 \\
\hline \multicolumn{11}{|l|}{ Transmission category ${ }^{d}$} \\
\hline \multicolumn{11}{|l|}{ Male adult or adolescent } \\
\hline Male-to-male sexual contact & 6,461 & - & 6,398 & - & 6,493 & - & 6,577 & - & 6,458 & - \\
\hline Injection drug use & 2,680 & - & 2,556 & - & 2,467 & - & 2,482 & - & 2,258 & - \\
\hline $\begin{array}{l}\text { Male-to-male sexual contact and } \\
\text { injection drug use }\end{array}$ & 1,316 & - & 1,291 & - & 1,311 & - & 1,323 & - & 1,325 & - \\
\hline Heterosexual contact ${ }^{\mathrm{e}}$ & 1,713 & - & 1,625 & - & 1,614 & - & 1,567 & - & 1,495 & - \\
\hline Perinatal & 37 & - & 43 & - & 37 & - & 32 & - & 28 & - \\
\hline Other $^{f}$ & 88 & - & 79 & - & 78 & - & 64 & - & 74 & - \\
\hline Subtotal & 12,296 & 9.7 & 11,992 & 9.4 & 12,000 & 9.3 & 12,045 & 9.3 & 11,638 & 8.9 \\
\hline
\end{tabular}


Table 16a. Deaths of persons with diagnosed HIV infection, by year of death and selected characteristics, 2011-2015-United States (cont)

\begin{tabular}{|c|c|c|c|c|c|c|c|c|c|c|}
\hline & \multicolumn{2}{|c|}{2011} & \multicolumn{2}{|c|}{2012} & \multicolumn{2}{|c|}{2013} & \multicolumn{2}{|c|}{2014} & \multicolumn{2}{|c|}{2015} \\
\hline & No. & Rate $^{a}$ & No. & Rate $^{a}$ & No. & Rate $^{a}$ & No. & Rate $^{a}$ & No. & Rate $^{a}$ \\
\hline \multicolumn{11}{|c|}{ Female adult or adolescent } \\
\hline Injection drug use & 1,612 & - & 1,510 & - & 1,518 & - & 1,470 & - & 1,337 & - \\
\hline Heterosexual contact $\mathrm{e}^{\mathrm{e}}$ & 2,606 & - & 2,698 & - & 2,520 & - & 2,674 & - & 2,407 & - \\
\hline Perinatal & 48 & - & 43 & - & 32 & - & 31 & - & 46 & - \\
\hline Other $^{f}$ & 33 & - & 37 & - & 32 & - & 33 & - & 26 & - \\
\hline Subtotal & 4,300 & 3.2 & 4,288 & 3.2 & 4,102 & 3.0 & 4,208 & 3.1 & 3,816 & 2.8 \\
\hline \multicolumn{11}{|c|}{ Child (<13 yrs at death) } \\
\hline Perinatal & 1 & - & 3 & - & 1 & - & 1 & - & 2 & - \\
\hline Other $^{f}$ & 0 & - & 1 & - & 0 & - & 0 & - & 2 & - \\
\hline Subtotal & 1 & 0.0 & 4 & 0.0 & 1 & 0.0 & 1 & 0.0 & 4 & 0.0 \\
\hline \multicolumn{11}{|l|}{ Region of residence ${ }^{g}$} \\
\hline Northeast & 4,248 & 7.6 & 3,915 & 7.0 & 3,950 & 7.1 & 3,805 & 6.8 & 3,585 & 6.4 \\
\hline Midwest & 1,887 & 2.8 & 1,906 & 2.8 & 1,775 & 2.6 & 1,790 & 2.6 & 1,741 & 2.6 \\
\hline South & 7,955 & 6.9 & 7,976 & 6.8 & 7,836 & 6.6 & 8,071 & 6.7 & 7,602 & 6.3 \\
\hline West & 2,507 & 3.4 & 2,487 & 3.4 & 2,542 & 3.4 & 2,588 & 3.4 & 2,530 & 3.3 \\
\hline Total $^{\mathrm{h}}$ & 16,597 & 5.3 & 16,284 & 5.2 & 16,103 & 5.1 & 16,254 & 5.1 & 15,458 & 4.8 \\
\hline
\end{tabular}



${ }^{a}$ Rates are per 100,000 population. Rates are not calculated by transmission category because of the lack of denominator data.

${ }^{b}$ Includes Asian/Pacific Islander legacy cases (see Technical Notes).

${ }^{\mathrm{C}}$ Hispanics/Latinos can be of any race.

${ }^{d}$ Data have been statistically adjusted to account for missing transmission category; therefore, values may not sum to column subtotals and total.

${ }^{\mathrm{e}}$ Heterosexual contact with a person known to have, or to be at high risk for, HIV infection.

$\mathrm{f}$ Includes hemophilia, blood transfusion, and risk factor not reported or not identified.

9 Data are based on residence at death. When information on residence at death was not available, state at death (where a person's death occurred) was used.

$\mathrm{h}$ Includes persons whose race/ethnicity is unknown. 


\begin{tabular}{|c|c|c|c|c|c|c|c|c|c|c|}
\hline & \multicolumn{2}{|c|}{2011} & \multicolumn{2}{|c|}{2012} & \multicolumn{2}{|c|}{2013} & \multicolumn{2}{|c|}{2014} & \multicolumn{2}{|c|}{2015} \\
\hline & No. & Rate $^{a}$ & No. & Rate $^{a}$ & No. & Rate $^{a}$ & No. & Rate $^{a}$ & No. & Rate $^{a}$ \\
\hline \multicolumn{11}{|l|}{ Age at death (yr) } \\
\hline$<13$ & 1 & 0.0 & 4 & 0.0 & 1 & 0.0 & 1 & 0.0 & 4 & 0.0 \\
\hline $13-14$ & 2 & 0.0 & 2 & 0.0 & 1 & 0.0 & 3 & 0.0 & 0 & 0.0 \\
\hline $15-19$ & 26 & 0.1 & 24 & 0.1 & 11 & 0.1 & 13 & 0.1 & 12 & 0.1 \\
\hline $20-24$ & 207 & 0.9 & 178 & 0.8 & 173 & 0.7 & 173 & 0.7 & 145 & 0.6 \\
\hline $25-29$ & 432 & 2.0 & 430 & 2.0 & 435 & 2.0 & 373 & 1.7 & 395 & 1.7 \\
\hline 30-34 & 708 & 3.4 & 734 & 3.5 & 656 & 3.0 & 660 & 3.0 & 601 & 2.8 \\
\hline 35-39 & 1,110 & 5.6 & 1,003 & 5.1 & 889 & 4.5 & 909 & 4.5 & 806 & 3.9 \\
\hline $40-44$ & 1,920 & 9.0 & 1,687 & 7.9 & 1,516 & 7.2 & 1,395 & 6.7 & 1,239 & 6.1 \\
\hline $45-49$ & 3,039 & 13.5 & 2,742 & 12.5 & 2,549 & 11.9 & 2,265 & 10.7 & 1,979 & 9.4 \\
\hline $50-54$ & 3,256 & 14.3 & 3,225 & 14.1 & 3,186 & 14.0 & 3,096 & 13.6 & 2,974 & 13.2 \\
\hline $55-59$ & 2,744 & 13.4 & 2,727 & 13.0 & 2,819 & 13.1 & 3,011 & 13.9 & 2,837 & 12.9 \\
\hline $60-64$ & 1,792 & 9.9 & 1,923 & 10.7 & 2,010 & 11.0 & 2,286 & 12.2 & 2,154 & 11.2 \\
\hline$\geq 65$ & 1,903 & 4.5 & 2,075 & 4.7 & 2,326 & 5.1 & 2,538 & 5.4 & 2,703 & 5.6 \\
\hline \multicolumn{11}{|l|}{ Race/ethnicity } \\
\hline American Indian/Alaska Native & 58 & - & 54 & - & 64 & - & 61 & - & 58 & - \\
\hline Asian ${ }^{b}$ & 89 & - & 74 & - & 73 & - & 64 & - & 77 & - \\
\hline Black/African American & 7,922 & - & 7,693 & - & 7,492 & - & 7,429 & - & 6,965 & - \\
\hline Hispanic/Latinoc & 3,264 & - & 2,940 & - & 3,108 & - & 3,223 & - & 2,863 & - \\
\hline Native Hawaiian/Other Pacific Islander & 12 & - & 7 & - & 12 & - & 8 & - & 7 & - \\
\hline White & 4,991 & - & 5,123 & - & 4,911 & - & 5,017 & - & 4,916 & - \\
\hline Multiple races & 802 & - & 841 & - & 908 & - & 917 & - & 958 & - \\
\hline \multicolumn{10}{|l|}{ Transmission category ${ }^{\mathrm{d}}$} & \\
\hline Male-to-male sexual contact & 6,546 & - & 6,456 & - & 6,577 & - & 6,663 & - & 6,531 & - \\
\hline Injection drug use & 2,892 & - & 2,724 & - & 2,613 & - & 2,624 & - & 2,383 & - \\
\hline $\begin{array}{l}\text { Male-to-male sexual contact and } \\
\text { injection drug use }\end{array}$ & 1,348 & - & 1,320 & - & 1,338 & - & 1,350 & - & 1,355 & - \\
\hline Heterosexual contact ${ }^{\mathrm{e}}$ & 1,792 & - & 1,714 & - & 1,700 & - & 1,648 & - & 1,555 & - \\
\hline Perinatal & 38 & - & 45 & - & 39 & - & 32 & - & 29 & - \\
\hline Other $^{f}$ & 88 & - & 80 & - & 78 & - & 66 & - & 75 & - \\
\hline Subtotal & 12,704 & 9.9 & 12,339 & 9.6 & 12,346 & 9.5 & 12,382 & 9.4 & 11,928 & 9.0 \\
\hline
\end{tabular}




\begin{tabular}{|c|c|c|c|c|c|c|c|c|c|c|}
\hline & \multicolumn{2}{|c|}{2011} & \multicolumn{2}{|c|}{2012} & \multicolumn{2}{|c|}{2013} & \multicolumn{2}{|c|}{2014} & \multicolumn{2}{|c|}{2015} \\
\hline & No. & Rate $^{a}$ & No. & Rate $^{a}$ & No. & Rate $^{a}$ & No. & Rate $^{a}$ & No. & Rate $^{\mathrm{a}}$ \\
\hline \multicolumn{11}{|c|}{ Female adult or adolescent } \\
\hline Injection drug use & 1,658 & - & 1,543 & - & 1,560 & - & 1,510 & - & 1,358 & - \\
\hline Heterosexual contact ${ }^{\mathrm{e}}$ & 2,693 & - & 2,783 & - & 2,600 & - & 2,765 & - & 2,486 & - \\
\hline Perinatal & 50 & - & 45 & - & 33 & - & 32 & - & 47 & - \\
\hline Other ${ }^{f}$ & 34 & - & 39 & - & 32 & - & 33 & - & 26 & - \\
\hline Subtotal & 4,435 & 3.3 & 4,411 & 3.3 & 4,225 & 3.1 & 4,340 & 3.2 & 3,917 & 2.8 \\
\hline \multicolumn{11}{|l|}{ Child (<13 yrs at death) } \\
\hline Perinatal & 1 & - & 3 & - & 1 & - & 1 & - & 2 & - \\
\hline Other ${ }^{f}$ & 0 & - & 1 & - & 0 & - & 0 & - & 2 & - \\
\hline Subtotal & 1 & 0.0 & 4 & 0.0 & 1 & 0.0 & 1 & 0.0 & 4 & 0.0 \\
\hline \multicolumn{11}{|l|}{ Region of residence $^{g}$} \\
\hline Northeast & 4,248 & 7.6 & 3,915 & 7.0 & 3,950 & 7.1 & 3,805 & 6.8 & 3,585 & 6.4 \\
\hline Midwest & 1,887 & 2.8 & 1,906 & 2.8 & 1,775 & 2.6 & 1,790 & 2.6 & 1,741 & 2.6 \\
\hline South & 7,955 & 6.9 & 7,976 & 6.8 & 7,836 & 6.6 & 8,071 & 6.7 & 7,602 & 6.3 \\
\hline West & 2,507 & 3.4 & 2,487 & 3.4 & 2,542 & 3.4 & 2,588 & 3.4 & 2,530 & 3.3 \\
\hline U.S. dependent areas & 543 & 13.3 & 470 & 11.7 & 469 & 11.8 & 469 & 11.9 & 391 & 10.1 \\
\hline Total $^{\mathrm{h}}$ & 17,140 & 5.4 & 16,754 & 5.3 & 16,572 & 5.2 & 16,723 & 5.2 & 15,849 & 4.9 \\
\hline
\end{tabular}




because of the lack of denominator data.

$\mathrm{b}$ Includes Asian/Pacific Islander legacy cases (see Technical Notes).

${ }^{C}$ Hispanics/Latinos can be of any race.

${ }^{d}$ Data have been statistically adjusted to account for missing transmission category; therefore, values may not sum to column subtotals and total.

${ }^{\text {e }}$ Heterosexual contact with a person known to have, or to be at high risk for, HIV infection.

$\mathrm{f}$ Includes hemophilia, blood transfusion, and risk factor not reported or not identified.

9 Data are based on residence at death. When information on residence at death was not available, state at death (where a person's death occurred) was used.

$\mathrm{h}$ Includes persons whose race/ethnicity is unknown. 


\begin{tabular}{|c|c|c|c|c|c|c|c|c|c|c|c|}
\hline & \multicolumn{2}{|c|}{2011} & \multicolumn{2}{|c|}{2012} & \multicolumn{2}{|c|}{2013} & \multicolumn{2}{|c|}{2014} & \multicolumn{2}{|c|}{2015} & \multirow{2}{*}{$\begin{array}{c}\text { Cumulative }^{\mathrm{a}} \\
\text { No. }\end{array}$} \\
\hline & No. & Rate $^{b}$ & No. & Rate $^{b}$ & No. & Rate $^{b}$ & No. & Rate $^{\mathrm{b}}$ & No. & Rate $^{\mathrm{b}}$ & \\
\hline \multicolumn{12}{|l|}{ Age at death (yr) } \\
\hline$<13$ & 0 & 0.0 & 1 & 0.0 & 1 & 0.0 & 0 & 0.0 & 2 & 0.0 & 4,968 \\
\hline $13-14$ & 1 & 0.0 & 1 & 0.0 & 0 & 0.0 & 1 & 0.0 & 0 & 0.0 & 296 \\
\hline 15-19 & 20 & 0.1 & 16 & 0.1 & 6 & 0.0 & 8 & 0.0 & 7 & 0.0 & 1,313 \\
\hline $20-24$ & 149 & 0.7 & 131 & 0.6 & 123 & 0.5 & 116 & 0.5 & 106 & 0.5 & 10,077 \\
\hline $25-29$ & 317 & 1.5 & 320 & 1.5 & 315 & 1.5 & 270 & 1.2 & 296 & 1.3 & 47,563 \\
\hline $30-34$ & 546 & 2.7 & 559 & 2.7 & 512 & 2.4 & 493 & 2.3 & 446 & 2.1 & 102,737 \\
\hline $35-39$ & 893 & 4.6 & 781 & 4.0 & 704 & 3.6 & 714 & 3.6 & 631 & 3.1 & 129,420 \\
\hline 40-44 & 1,545 & 7.3 & 1,355 & 6.4 & 1,224 & 5.9 & 1,115 & 5.4 & 976 & 4.8 & 124,221 \\
\hline $45-49$ & 2,467 & 11.1 & 2,249 & 10.4 & 2,050 & 9.7 & 1,820 & 8.7 & 1,602 & 7.7 & 98,819 \\
\hline $50-54$ & 2,622 & 11.6 & 2,592 & 11.5 & 2,570 & 11.4 & 2,511 & 11.1 & 2,422 & 10.9 & 70,633 \\
\hline $55-59$ & 2,183 & 10.8 & 2,190 & 10.5 & 2,265 & 10.7 & 2,368 & 11.0 & 2,286 & 10.5 & 46,000 \\
\hline $60-64$ & 1,404 & 7.9 & 1,509 & 8.5 & 1,592 & 8.8 & 1,805 & 9.7 & 1,698 & 8.9 & 27,909 \\
\hline$\geq 65$ & 1,409 & 3.4 & 1,542 & 3.6 & 1,750 & 3.9 & 1,908 & 4.1 & 2,025 & 4.2 & 28,833 \\
\hline \multicolumn{12}{|l|}{ Race/ethnicity } \\
\hline American Indian/Alaska Native & 48 & 2.1 & 41 & 1.8 & 52 & 2.2 & 43 & 1.8 & 49 & 2.1 & 1,988 \\
\hline Asian ${ }^{\mathrm{C}}$ & 69 & 0.5 & 61 & 0.4 & 64 & 0.4 & 47 & 0.3 & 62 & 0.4 & 3,542 \\
\hline Black/African American & 6,415 & 16.7 & 6,233 & 16.1 & 6,038 & 15.4 & 5,974 & 15.1 & 5,586 & 14.0 & 285,744 \\
\hline Hispanic/Latino $^{d}$ & 2,316 & 4.5 & 2,119 & 4.0 & 2,257 & 4.2 & 2,335 & 4.2 & 2,100 & 3.7 & 106,644 \\
\hline Native Hawaiian/Other Pacific Islander & 9 & 1.8 & 6 & 1.2 & 8 & 1.5 & 5 & 0.9 & 4 & 0.7 & 369 \\
\hline White & 3,993 & 2.0 & 4,065 & 2.1 & 3,904 & 2.0 & 3,950 & 2.0 & 3,879 & 2.0 & 279,807 \\
\hline Multiple races & 706 & 12.1 & 720 & 12.0 & 788 & 12.7 & 775 & 12.1 & 817 & 12.4 & 14,647 \\
\hline \multicolumn{12}{|l|}{ Transmission category ${ }^{\mathrm{e}}$} \\
\hline \multicolumn{12}{|l|}{ Male adult or adolescent } \\
\hline Male-to-male sexual contact & 5,248 & - & 5,188 & - & 5,228 & - & 5,269 & - & 5,180 & - & 325,330 \\
\hline Injection drug use & 2,179 & - & 2,097 & - & 2,032 & - & 2,032 & - & 1,869 & - & 134,466 \\
\hline $\begin{array}{l}\text { Male-to-male sexual contact and } \\
\text { injection drug use }\end{array}$ & 1,125 & - & 1,105 & - & 1,119 & - & 1,137 & - & 1,124 & - & 53,521 \\
\hline Heterosexual contact ${ }^{f}$ & 1,373 & - & 1,317 & - & 1,290 & - & 1,268 & - & 1,192 & - & 38,727 \\
\hline Perinatal & 34 & - & 40 & - & 32 & - & 28 & - & 26 & - & 463 \\
\hline Otherg & 74 & - & 65 & - & 60 & - & 55 & - & 62 & - & 8,789 \\
\hline Subtotal & 10,032 & 7.9 & 9,812 & 7.7 & 9,762 & 7.6 & 9,789 & 7.5 & 9,453 & 7.2 & 561,296 \\
\hline
\end{tabular}


Table 17a. Deaths of persons with diagnosed HIV infection ever classified as stage 3 (AIDS), by year of death and selected characteristics, 2011-2015 and cumulative-United States (cont)

\begin{tabular}{|c|c|c|c|c|c|c|c|c|c|c|c|}
\hline & \multicolumn{2}{|c|}{2011} & \multicolumn{2}{|c|}{2012} & \multicolumn{2}{|c|}{2013} & \multicolumn{2}{|c|}{2014} & \multicolumn{2}{|c|}{2015} & \multirow{2}{*}{$\begin{array}{c}\text { Cumulative }^{a} \\
\text { No. }\end{array}$} \\
\hline & No. & Rate $^{b}$ & No. & Rate $^{b}$ & No. & Rate $^{b}$ & No. & Rate $^{b}$ & No. & Rate $^{b}$ & \\
\hline \multicolumn{12}{|c|}{ Female adult or adolescent } \\
\hline Injection drug use & 1,341 & - & 1,243 & - & 1,267 & - & 1,198 & - & 1,090 & - & 59,438 \\
\hline Heterosexual contact $^{f}$ & 2,113 & - & 2,117 & - & 2,025 & - & 2,086 & - & 1,885 & - & 62,839 \\
\hline Perinatal & 44 & - & 43 & - & 31 & - & 30 & - & 45 & - & 592 \\
\hline Otherg & 26 & - & 30 & - & 26 & - & 25 & - & 22 & - & 3,655 \\
\hline Subtotal & 3,524 & 2.7 & 3,433 & 2.6 & 3,349 & 2.5 & 3,340 & 2.5 & 3,042 & 2.2 & 126,525 \\
\hline \multicolumn{12}{|c|}{ Child (<13 yrs at death) } \\
\hline Perinatal & 0 & - & 1 & - & 1 & - & 0 & - & 0 & - & 4,507 \\
\hline Otherg & 0 & - & 0 & - & 0 & - & 0 & - & 2 & - & 461 \\
\hline Subtotal & 0 & 0.0 & 1 & 0.0 & 1 & 0.0 & 0 & 0.0 & 2 & 0.0 & 4,968 \\
\hline \multicolumn{12}{|l|}{ Region of residence $^{h}$} \\
\hline Northeast & 3,474 & 6.2 & 3,237 & 5.8 & 3,253 & 5.8 & 3,057 & 5.4 & 2,883 & 5.1 & 213,084 \\
\hline Midwest & 1,521 & 2.3 & 1,495 & 2.2 & 1,415 & 2.1 & 1,414 & 2.1 & 1,365 & 2.0 & 73,965 \\
\hline South & 6,483 & 5.6 & 6,449 & 5.5 & 6,336 & 5.4 & 6,515 & 5.4 & 6,159 & 5.1 & 272,756 \\
\hline West & 2,078 & 2.9 & 2,065 & 2.8 & 2,108 & 2.8 & 2,143 & 2.9 & 2,090 & 2.8 & 132,984 \\
\hline Total $^{\mathrm{i}}$ & 13,556 & 4.3 & 13,246 & 4.2 & 13,112 & 4.1 & 13,129 & 4.1 & 12,497 & 3.9 & 692,789 \\
\hline
\end{tabular}

Note. Deaths of persons with a diagnosis of HIV infection may be due to any cause. Numbers less than 12, and rates and trends based on these numbers, should be interpreted with caution.

a From the beginning of the epidemic through 2015.

${ }^{b}$ Rates are per 100,000 population. Rates are not calculated by transmission category because of the lack of denominator data.

${ }^{\mathrm{C}}$ Includes Asian/Pacific Islander legacy cases (see Technical Notes).

$\mathrm{d}_{\text {Hispanics/Latinos can be of any race. }}$

${ }^{\mathrm{e}}$ Data have been statistically adjusted to account for missing transmission category; therefore, values may not sum to column subtotals and total.

${ }^{f}$ Heterosexual contact with a person known to have, or to be at high risk for, HIV infection.

$\mathrm{g}$ Includes hemophilia, blood transfusion, and risk factor not reported or not identified.



i Includes persons whose race/ethnicity is unknown. 


\begin{tabular}{|c|c|c|c|c|c|c|c|c|c|c|c|}
\hline & \multicolumn{2}{|c|}{2011} & \multicolumn{2}{|c|}{2012} & \multicolumn{2}{|c|}{2013} & \multicolumn{2}{|c|}{2014} & \multicolumn{2}{|c|}{2015} & \multirow{2}{*}{$\begin{array}{c}\text { Cumulative }^{\mathrm{a}} \\
\text { No. }\end{array}$} \\
\hline & No. & Rate $^{b}$ & No. & Rate $^{b}$ & No. & Rate $^{b}$ & No. & Rate $^{\mathrm{b}}$ & No. & Rate $^{b}$ & \\
\hline \multicolumn{12}{|l|}{ Age at death (yr) } \\
\hline$<13$ & 0 & 0.0 & 1 & 0.0 & 1 & 0.0 & 0 & 0.0 & 2 & 0.0 & 5,210 \\
\hline $13-14$ & 1 & 0.0 & 1 & 0.0 & 0 & 0.0 & 1 & 0.0 & 0 & 0.0 & 306 \\
\hline $15-19$ & 21 & 0.1 & 17 & 0.1 & 8 & 0.0 & 8 & 0.0 & 7 & 0.0 & 1,383 \\
\hline $20-24$ & 151 & 0.7 & 134 & 0.6 & 126 & 0.5 & 118 & 0.5 & 108 & 0.5 & 10,606 \\
\hline 25-29 & 327 & 1.5 & 327 & 1.5 & 317 & 1.5 & 273 & 1.2 & 298 & 1.3 & 49,797 \\
\hline $30-34$ & 560 & 2.7 & 577 & 2.7 & 527 & 2.4 & 506 & 2.3 & 462 & 2.1 & 107,006 \\
\hline $35-39$ & 925 & 4.7 & 807 & 4.1 & 723 & 3.6 & 730 & 3.6 & 644 & 3.1 & 134,641 \\
\hline $40-44$ & 1,597 & 7.5 & 1,394 & 6.5 & 1,249 & 5.9 & 1,138 & 5.5 & 1,000 & 4.9 & 128,802 \\
\hline $45-49$ & 2,561 & 11.4 & 2,310 & 10.5 & 2,115 & 9.8 & 1,864 & 8.8 & 1,640 & 7.8 & 102,184 \\
\hline $50-54$ & 2,703 & 11.8 & 2,659 & 11.6 & 2,636 & 11.5 & 2,586 & 11.3 & 2,490 & 11.0 & 72,907 \\
\hline $55-59$ & 2,234 & 10.9 & 2,231 & 10.6 & 2,313 & 10.8 & 2,436 & 11.2 & 2,331 & 10.6 & 47,297 \\
\hline 60-64 & 1,438 & 8.0 & 1,544 & 8.6 & 1,631 & 8.9 & 1,851 & 9.9 & 1,735 & 9.0 & 28,794 \\
\hline$\geq 65$ & 1,456 & 3.5 & 1,587 & 3.6 & 1,798 & 4.0 & 1,970 & 4.2 & 2,070 & 4.3 & 29,973 \\
\hline \multicolumn{12}{|l|}{ Race/ethnicity } \\
\hline American Indian/Alaska Native & 48 & - & 41 & - & 52 & - & 43 & - & 49 & - & 1,988 \\
\hline Asian ${ }^{c}$ & 71 & - & 62 & - & 64 & - & 48 & - & 62 & - & 3,587 \\
\hline Black/African American & 6,423 & - & 6,242 & - & 6,041 & - & 5,978 & - & 5,589 & - & 286,031 \\
\hline Hispanic/Latino $^{d}$ & 2,721 & - & 2,450 & - & 2,580 & - & 2,682 & - & 2,384 & - & 132,300 \\
\hline Native Hawaiian/Other Pacific Islander & 11 & - & 6 & - & 10 & - & 5 & - & 5 & - & 384 \\
\hline White & 3,993 & - & 4,066 & - & 3,906 & - & 3,950 & - & 3,880 & - & 279,910 \\
\hline Multiple races & 707 & - & 721 & - & 790 & - & 775 & - & 818 & - & 14,658 \\
\hline \multicolumn{12}{|l|}{ Transmission category ${ }^{\mathrm{e}}$} \\
\hline \multicolumn{12}{|l|}{ Male adult or adolescent } \\
\hline Male-to-male sexual contact & 5,318 & - & 5,228 & - & 5,293 & - & 5,330 & - & 5,234 & - & 329,540 \\
\hline Injection drug use & 2,333 & - & 2,221 & - & 2,128 & - & 2,141 & - & 1,963 & - & 145,608 \\
\hline $\begin{array}{l}\text { Male-to-male sexual contact and } \\
\text { injection drug use }\end{array}$ & 1,154 & - & 1,131 & - & 1,143 & - & 1,159 & - & 1,149 & - & 55,568 \\
\hline Heterosexual contact ${ }^{f}$ & 1,434 & - & 1,376 & - & 1,353 & - & 1,321 & - & 1,235 & - & 41,359 \\
\hline Perinatal & 35 & - & 42 & - & 34 & - & 28 & - & 27 & - & 491 \\
\hline Otherg & 74 & - & 67 & - & 60 & - & 57 & - & 62 & - & 8,923 \\
\hline Subtotal & 10,348 & 8.1 & 10,065 & 7.8 & 10,011 & 7.7 & 10,036 & 7.6 & 9,670 & 7.3 & 581,490 \\
\hline
\end{tabular}


Table 17b. Deaths of persons with diagnosed HIV infection ever classified as stage 3 (AIDS), by year of death and selected characteristics, $2011-2015$ and cumulative-United States and 6 dependent areas (cont)

\begin{tabular}{|c|c|c|c|c|c|c|c|c|c|c|c|}
\hline & \multicolumn{2}{|c|}{2011} & \multicolumn{2}{|c|}{2012} & \multicolumn{2}{|c|}{2013} & \multicolumn{2}{|c|}{2014} & \multicolumn{2}{|c|}{2015} & \multirow{2}{*}{$\begin{array}{c}\text { Cumulative }^{2} \\
\text { No. }\end{array}$} \\
\hline & No. & Rate $^{b}$ & No. & Rate $^{b}$ & No. & Rate $^{b}$ & No. & Rate $^{b}$ & No. & Rate $^{\mathrm{b}}$ & \\
\hline \multicolumn{12}{|c|}{ Female adult or adolescent } \\
\hline Injection drug use & 1,380 & - & 1,266 & - & 1,297 & - & 1,234 & - & 1,103 & - & 61,676 \\
\hline Heterosexual contact ${ }^{f}$ & 2,174 & - & 2,180 & - & 2,077 & - & 2,155 & - & 1,944 & - & 66,145 \\
\hline Perinatal & 45 & - & 45 & - & 32 & - & 31 & - & 46 & - & 625 \\
\hline Other ${ }^{g}$ & 27 & - & 32 & - & 26 & - & 25 & - & 22 & - & 3,760 \\
\hline Subtotal & 3,626 & 2.7 & 3,523 & 2.6 & 3,432 & 2.5 & 3,445 & 2.5 & 3,115 & 2.2 & 132,206 \\
\hline \multicolumn{12}{|l|}{ Child (<13 yrs at death) } \\
\hline Perinatal & 0 & - & 1 & - & 1 & - & 0 & - & 0 & - & 4,734 \\
\hline Other ${ }^{g}$ & 0 & - & 0 & - & 0 & - & 0 & - & 2 & - & 476 \\
\hline Subtotal & 0 & 0.0 & 1 & 0.0 & 1 & 0.0 & 0 & 0.0 & 2 & 0.0 & 5,210 \\
\hline \multicolumn{12}{|l|}{ Region of residence $^{h}$} \\
\hline Northeast & 3,474 & 6.2 & 3,237 & 5.8 & 3,253 & 5.8 & 3,057 & 5.4 & 2,883 & 5.1 & 213,084 \\
\hline Midwest & 1,521 & 2.3 & 1,495 & 2.2 & 1,415 & 2.1 & 1,414 & 2.1 & 1,365 & 2.0 & 73,965 \\
\hline South & 6,483 & 5.6 & 6,449 & 5.5 & 6,336 & 5.4 & 6,515 & 5.4 & 6,159 & 5.1 & 272,756 \\
\hline West & 2,078 & 2.9 & 2,065 & 2.8 & 2,108 & 2.8 & 2,143 & 2.9 & 2,090 & 2.8 & 132,984 \\
\hline U.S. dependent areas & 418 & 10.3 & 343 & 8.5 & 332 & 8.3 & 352 & 9.0 & 290 & 7.5 & 26,117 \\
\hline Total $^{\mathrm{i}}$ & 13,974 & 4.4 & 13,589 & 4.3 & 13,444 & 4.2 & 13,481 & 4.2 & 12,787 & 3.9 & 718,906 \\
\hline
\end{tabular}

Note. Deaths of persons with a diagnosis of HIV infection may be due to any cause. Numbers less than 12, and rates and trends based on these numbers, should be interpreted with caution.

${ }^{a}$ From the beginning of the epidemic through 2015.


because of the lack of denominator data.

${ }^{c}$ Includes Asian/Pacific Islander legacy cases (see Technical Notes).

$\mathrm{d}$ Hispanics/Latinos can be of any race.

e Data have been statistically adjusted to account for missing transmission category; therefore, values may not sum to column subtotals and total.

f Heterosexual contact with a person known to have, or to be at high risk for, HIV infection.

$\mathrm{g}$ Includes hemophilia, blood transfusion, and risk factor not reported or not identified.

${ }^{h}$ Data are based on residence at death. When information on residence at death was not available, state at death (where a person's death occurred) was used.

i Includes persons whose race/ethnicity is unknown. 


\begin{tabular}{|c|c|c|c|c|c|c|c|c|c|c|c|c|c|c|c|c|}
\hline & \multicolumn{2}{|c|}{$\begin{array}{l}\text { American Indian/ } \\
\text { Alaska Native }\end{array}$} & \multicolumn{2}{|c|}{ Asian $^{a}$} & \multicolumn{2}{|c|}{$\begin{array}{l}\text { Black/African } \\
\text { American }\end{array}$} & \multicolumn{2}{|c|}{ Hispanic/Latino $^{b}$} & \multicolumn{2}{|c|}{$\begin{array}{l}\text { Native Hawaiian/ } \\
\text { Other Pacific } \\
\text { Islander }\end{array}$} & \multicolumn{2}{|c|}{ White } & \multicolumn{2}{|c|}{ Multiple races } & \multicolumn{2}{|c|}{ Total $^{c}$} \\
\hline & No. & Rate $^{d}$ & No. & Rate $^{d}$ & No. & Rate $^{d}$ & No. & Rate $^{d}$ & No. & Rate $^{d}$ & No. & Rate $^{d}$ & No. & Rate $^{d}$ & No. & Rate $^{d}$ \\
\hline \multicolumn{17}{|l|}{ Age at death (yr) } \\
\hline$<13$ & 0 & 0.0 & 0 & 0.0 & 3 & 0.0 & 0 & 0.0 & 0 & 0.0 & 1 & 0.0 & 0 & 0.0 & 4 & 0.0 \\
\hline 13-14 & 0 & 0.0 & 0 & 0.0 & 0 & 0.0 & 0 & 0.0 & 0 & 0.0 & 0 & 0.0 & 0 & 0.0 & 0 & 0.0 \\
\hline 15-19 & 0 & 0.0 & 0 & 0.0 & 7 & 0.2 & 1 & 0.0 & 0 & 0.0 & 4 & 0.0 & 0 & 0.0 & 12 & 0.1 \\
\hline 20-24 & 0 & 0.0 & 1 & 0.1 & 98 & 2.9 & 18 & 0.4 & 0 & 0.0 & 16 & 0.1 & 10 & 1.7 & 143 & 0.6 \\
\hline $25-29$ & 1 & 0.5 & 3 & 0.2 & 225 & 7.2 & 74 & 1.6 & 1 & 1.9 & 63 & 0.5 & 24 & 5.2 & 391 & 1.7 \\
\hline 30-34 & 6 & 3.7 & 7 & 0.5 & 294 & 10.6 & 121 & 2.7 & 0 & 0.0 & 117 & 0.9 & 36 & 9.4 & 581 & 2.7 \\
\hline $35-39$ & 5 & 3.4 & 8 & 0.6 & 400 & 15.4 & 163 & 3.9 & 1 & 2.4 & 170 & 1.5 & 40 & 12.3 & 787 & 3.9 \\
\hline $40-44$ & 5 & 3.5 & 11 & 0.8 & 554 & 21.8 & 229 & 5.9 & 1 & 2.7 & 331 & 2.8 & 70 & 25.1 & 1,201 & 6.0 \\
\hline $45-49$ & 8 & 5.4 & 12 & 1.0 & 820 & 31.8 & 352 & 10.2 & 0 & 0.0 & 615 & 4.7 & 121 & 48.7 & 1,931 & 9.3 \\
\hline 50-54 & 13 & 8.0 & 13 & 1.2 & 1,185 & 43.7 & 473 & 15.7 & 1 & 2.9 & 1,014 & 6.8 & 181 & 75.1 & 2,881 & 12.9 \\
\hline $55-59$ & 10 & 6.5 & 7 & 0.7 & 1,229 & 48.1 & 383 & 15.8 & 1 & 3.3 & 956 & 6.2 & 189 & 86.5 & 2,776 & 12.8 \\
\hline $60-64$ & 4 & 3.2 & 5 & 0.6 & 1,011 & 49.1 & 299 & 16.4 & 0 & 0.0 & 644 & 4.6 & 146 & 85.2 & 2,109 & 11.1 \\
\hline$\geq 65$ & 6 & 2.4 & 9 & 0.5 & 1,134 & 27.0 & 368 & 9.8 & 1 & 2.2 & 984 & 2.6 & 140 & 40.8 & 2,642 & 5.5 \\
\hline \multicolumn{17}{|l|}{ Transmission category ${ }^{\mathrm{e}}$} \\
\hline \multicolumn{17}{|l|}{ Male adult or adolescent } \\
\hline Male-to-male sexual contact & 20 & - & 53 & - & 2,037 & - & 1,050 & - & 4 & - & 2,950 & - & 341 & - & 6,458 & - \\
\hline Injection drug use & 5 & - & 3 & - & 1,170 & - & 479 & - & 0 & - & 438 & - & 164 & - & 2,258 & - \\
\hline $\begin{array}{l}\text { Male-to-male sexual contact } \\
\text { and injection drug use }\end{array}$ & 10 & - & 4 & - & 407 & - & 221 & - & 0 & - & 543 & - & 138 & - & 1,325 & - \\
\hline Heterosexual contact ${ }^{f}$ & 7 & - & 9 & - & 973 & - & 215 & - & 2 & - & 218 & - & 70 & - & 1,495 & - \\
\hline Perinatal & 0 & - & 1 & - & 16 & - & 5 & - & 0 & - & 3 & - & 3 & - & 28 & - \\
\hline Other ${ }^{g}$ & 0 & - & 0 & - & 19 & - & 10 & - & 0 & - & 37 & - & 8 & - & 74 & - \\
\hline Subtotal & 41 & 4.4 & 70 & 1.0 & 4,622 & 30.0 & 1,980 & 9.1 & 6 & 2.6 & 4,190 & 5.0 & 724 & 35.3 & 11,638 & 8.9 \\
\hline
\end{tabular}


Table 18a. Deaths of persons with diagnosed HIV infection, by race/ethnicity and selected characteristics, 2015-United States (cont)

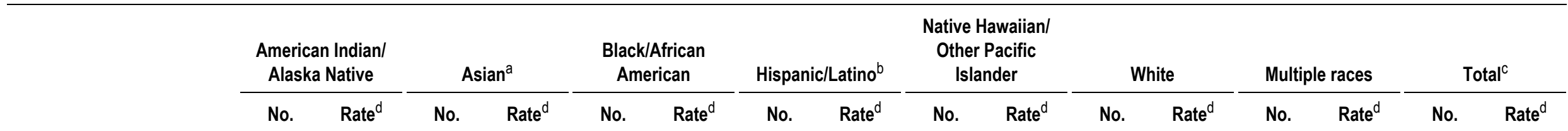

Female adult or adolescent

Injection drug use

Heterosexual contact ${ }^{f}$

Perinatal

Otherg

Subtotal

$\begin{array}{rrr}9 & - & 0 \\ 8 & - & 6 \\ 0 & - & 0 \\ 1 & - & 0 \\ 17 & 1.7 & 6\end{array}$

$\begin{array}{rr}- & 662 \\ - & 1,631 \\ - & 32 \\ - & 10 \\ 0.1 & 2,335\end{array}$

$\begin{array}{rrrr}- & 215 & - & 0 \\ - & 274 & - & 0 \\ - & 7 & - & 0 \\ - & 5 & - & 0 \\ 13.5 & 501 & 2.4 & 0\end{array}$

$\begin{array}{rrrrrrr}- & 343 & - & 108 & - & 1,337 & - \\ - & 366 & - & 122 & - & 2,407 & - \\ - & 6 & - & 1 & - & 46 & - \\ - & 9 & - & 2 & - & 26 & - \\ 0.0 & 724 & 0.8 & 233 & 10.6 & 3,816 & 2.8\end{array}$

\section{Child (<13 yrs at death)}

Perinatal

Other ${ }^{9}$

Subtotal

$$
0
$$

Region of residence ${ }^{\mathrm{h}}$

Northeast

Midwest

South

West

Total

$$
\begin{array}{ll}
- & 0 \\
- & 0 \\
0.0 & 0
\end{array}
$$$$
\text { - }
$$

0

0.0

1
2
3

$$
-
$$

$\begin{array}{lll}0 & - & 0 \\ 0 & - & 0 \\ 0 & 0.0 & 0\end{array}$

-
-
0.0

$\begin{array}{ll}1 & - \\ 0 & - \\ 1 & 0.0\end{array}$

$\begin{array}{cccc}0 & - & 2 & - \\ 0 & - & 2 & - \\ 0 & 0.0 & 4 & 0.0\end{array}$

Note. Deaths of persons with a diagnosis of HIV infection may be due to any cause. Numbers less than 12 , and rates based on these numbers, should be interpreted with caution.

a Includes Asian/Pacific Islander legacy cases (see Technical Notes).

${ }^{b}$ Hispanics/Latinos can be of any race.

${ }^{c}$ Includes persons whose race/ethnicity is unknown.

d Rates are per 100,000 population. Rates are not calculated by transmission category because of the lack of denominator data.

e Data have been statistically adjusted to account for missing transmission category; therefore, values may not sum to column subtotals and total.

${ }^{f}$ Heterosexual contact with a person known to have, or to be at high risk for, HIV infection.

$g$ Includes hemophilia, blood transfusion, and risk factor not reported or not identified.

${ }^{h}$ Data are based on residence at death. When information on residence at death was not available, state at death (where a person's death occurred) was used. 


\begin{tabular}{|c|c|c|c|c|c|c|c|c|}
\hline & $\begin{array}{c}\text { American Indian/ } \\
\text { Alaska Native } \\
\text { No. }\end{array}$ & $\begin{array}{c}\text { Asian }^{a} \\
\text { No. }\end{array}$ & $\begin{array}{c}\text { Black/African } \\
\text { American } \\
\text { No. } \\
\end{array}$ & $\begin{array}{c}\text { Hispanic/Latino }^{b} \\
\text { No. }\end{array}$ & $\begin{array}{c}\text { Native Hawaiian/ } \\
\text { Other Pacific } \\
\text { Islander } \\
\text { No. }\end{array}$ & $\begin{array}{l}\text { White } \\
\text { No. }\end{array}$ & $\begin{array}{c}\text { Multiple races } \\
\text { No. }\end{array}$ & $\begin{array}{c}\text { Total }^{\mathrm{C}} \\
\text { No. }\end{array}$ \\
\hline \multicolumn{9}{|l|}{ Age at death (yr) } \\
\hline$<13$ & 0 & 0 & 3 & 0 & 0 & 1 & 0 & 4 \\
\hline 13-14 & 0 & 0 & 0 & 0 & 0 & 0 & 0 & 0 \\
\hline 15-19 & 0 & 0 & 7 & 1 & 0 & 4 & 0 & 12 \\
\hline $20-24$ & 0 & 1 & 98 & 20 & 0 & 16 & 10 & 145 \\
\hline $25-29$ & 1 & 3 & 225 & 78 & 1 & 63 & 24 & 395 \\
\hline 30-34 & 6 & 8 & 294 & 140 & 0 & 117 & 36 & 601 \\
\hline $35-39$ & 5 & 8 & 400 & 181 & 1 & 170 & 41 & 806 \\
\hline 40-44 & 5 & 11 & 555 & 266 & 1 & 331 & 70 & 1,239 \\
\hline $45-49$ & 8 & 12 & 820 & 399 & 0 & 616 & 121 & 1,979 \\
\hline $50-54$ & 13 & 13 & 1,187 & 563 & 2 & 1,014 & 181 & 2,974 \\
\hline $55-59$ & 10 & 7 & 1,229 & 444 & 1 & 956 & 189 & 2,837 \\
\hline $60-64$ & 4 & 5 & 1,011 & 344 & 0 & 644 & 146 & 2,154 \\
\hline$\geq 65$ & 6 & 9 & 1,136 & 427 & 1 & 984 & 140 & 2,703 \\
\hline \multicolumn{9}{|l|}{ Transmission category ${ }^{d}$} \\
\hline \multicolumn{9}{|l|}{ Male adult or adolescent } \\
\hline Male-to-male sexual contact & 20 & 53 & 2,037 & 1,120 & 5 & 2,951 & 342 & 6,531 \\
\hline Injection drug use & 5 & 3 & 1,171 & 603 & 0 & 438 & 164 & 2,383 \\
\hline Male-to-male sexual contact and injection drug use & 10 & 4 & 407 & 251 & 0 & 543 & 138 & 1,355 \\
\hline Heterosexual contact ${ }^{e}$ & 7 & 9 & 975 & 275 & 2 & 218 & 70 & 1,555 \\
\hline Perinatal & 0 & 1 & 16 & 6 & 0 & 3 & 3 & 29 \\
\hline Other $^{f}$ & 0 & 0 & 19 & 11 & 0 & 37 & 8 & 75 \\
\hline Subtotal & 41 & 70 & 4,624 & 2,265 & 7 & 4,191 & 725 & 11,928 \\
\hline
\end{tabular}


Table 18b. Deaths of persons with diagnosed HIV infection, by race/ethnicity and selected characteristics, 2015-United States and 6 dependent areas (cont)

\begin{tabular}{|c|c|c|c|c|c|c|c|c|}
\hline & $\begin{array}{c}\text { American Indian/ } \\
\text { Alaska Native } \\
\text { No. }\end{array}$ & $\begin{array}{c}\text { Asian }^{a} \\
\text { No. }\end{array}$ & $\begin{array}{c}\text { Black/African } \\
\text { American } \\
\text { No. }\end{array}$ & $\begin{array}{c}\text { Hispanic/Latino }{ }^{b} \\
\text { No. }\end{array}$ & $\begin{array}{c}\text { Native Hawaiian/ } \\
\text { Other Pacific } \\
\text { Islander } \\
\text { No. }\end{array}$ & $\begin{array}{c}\text { White } \\
\text { No. }\end{array}$ & $\begin{array}{c}\text { Multiple races } \\
\text { No. }\end{array}$ & $\begin{array}{c}\text { Total }^{\mathrm{C}} \\
\text { No. }\end{array}$ \\
\hline \multicolumn{9}{|c|}{ Female adult or adolescent } \\
\hline Injection drug use & 9 & 0 & 662 & 236 & 0 & 343 & 108 & 1,358 \\
\hline Heterosexual contact ${ }^{e}$ & 8 & 7 & 1,634 & 350 & 0 & 366 & 122 & 2,486 \\
\hline Perinatal & 0 & 0 & 32 & 8 & 0 & 6 & 1 & 47 \\
\hline Other $^{f}$ & 1 & 0 & 10 & 5 & 0 & 9 & 2 & 26 \\
\hline Subtotal & 17 & 7 & 2,338 & 598 & 0 & 724 & 233 & 3,917 \\
\hline \multicolumn{9}{|c|}{ Child ( $<13$ yrs at end of year) } \\
\hline Perinatal & 0 & 0 & 1 & 0 & 0 & 1 & 0 & 2 \\
\hline Other $^{f}$ & 0 & 0 & 2 & 0 & 0 & 0 & 0 & 2 \\
\hline Subtotal & 0 & 0 & 3 & 0 & 0 & 1 & 0 & 4 \\
\hline \multicolumn{9}{|l|}{ Region of residence ${ }^{g}$} \\
\hline Northeast & 0 & 12 & 1,471 & 929 & 1 & 817 & 355 & 3,585 \\
\hline Midwest & 9 & 4 & 768 & 125 & 0 & 737 & 98 & 1,741 \\
\hline South & 22 & 17 & 4,242 & 836 & 1 & 2,129 & 350 & 7,602 \\
\hline West & 27 & 43 & 479 & 591 & 4 & 1,232 & 154 & 2,530 \\
\hline U.S. dependent areas & 0 & 1 & 5 & 382 & 1 & 1 & 1 & 391 \\
\hline Total & 58 & 77 & 6,965 & 2,863 & 7 & 4,916 & 958 & 15,849 \\
\hline
\end{tabular}

Note. Deaths of persons with a diagnosis of HIV infection may be due to any cause. Numbers less than 12 should be interpreted with caution.

a Includes Asian/Pacific Islander legacy cases (see Technical Notes).

${ }^{b}$ Hispanics/Latinos can be of any race.

${ }^{c}$ Includes persons whose race/ethnicity is unknown.

${ }^{d}$ Data have been statistically adjusted to account for missing transmission category; therefore, values may not sum to column subtotals and total.

e Heterosexual contact with a person known to have, or to be at high risk for, HIV infection.

f Includes hemophilia, blood transfusion, and risk factor not reported or not identified.

9 Data are based on residence at death. When information on residence at death was not available, state at death (where a person's death occurred) was used. 


\begin{tabular}{|c|c|c|c|c|c|c|c|c|c|c|c|c|c|c|c|c|}
\hline & \multicolumn{2}{|c|}{$\begin{array}{l}\text { American Indian/ } \\
\text { Alaska Native }\end{array}$} & \multicolumn{2}{|c|}{ Asian $^{\mathrm{a}}$} & \multicolumn{2}{|c|}{$\begin{array}{l}\text { Black/African } \\
\text { American }\end{array}$} & \multicolumn{2}{|c|}{ Hispanic/Latino ${ }^{b}$} & \multicolumn{2}{|c|}{$\begin{array}{l}\text { Native Hawaiian/ } \\
\text { Other Pacific } \\
\text { Islander }\end{array}$} & \multicolumn{2}{|c|}{ White } & \multicolumn{2}{|c|}{ Multiple races } & \multicolumn{2}{|c|}{ Total } \\
\hline & No. & Rate $^{\mathrm{C}}$ & No. & Rate $^{\mathrm{C}}$ & No. & Rate $^{c}$ & No. & Rate $^{\mathrm{C}}$ & No. & Rate $^{\mathrm{c}}$ & No. & Rate $^{\mathrm{c}}$ & No. & Rate $^{c}$ & No. & Rate \\
\hline \multicolumn{17}{|l|}{ Age at death (yr) } \\
\hline$<13$ & 0 & 0.0 & 0 & 0.0 & 2 & 0.0 & 0 & 0.0 & 0 & 0.0 & 0 & 0.0 & 0 & 0.0 & 2 & 0.0 \\
\hline $13-14$ & 0 & 0.0 & 0 & 0.0 & 0 & 0.0 & 0 & 0.0 & 0 & 0.0 & 0 & 0.0 & 0 & 0.0 & 0 & 0.0 \\
\hline 15-19 & 0 & 0.0 & 0 & 0.0 & 4 & 0.1 & 0 & 0.0 & 0 & 0.0 & 3 & 0.0 & 0 & 0.0 & 7 & 0.0 \\
\hline $20-24$ & 0 & 0.0 & 0 & 0.0 & 75 & 2.2 & 14 & 0.3 & 0 & 0.0 & 11 & 0.1 & 6 & 1.0 & 106 & 0.5 \\
\hline $25-29$ & 1 & 0.5 & 2 & 0.1 & 164 & 5.3 & 63 & 1.4 & 0 & 0.0 & 44 & 0.3 & 22 & 4.8 & 296 & 1.3 \\
\hline 30-34 & 5 & 3.1 & 5 & 0.3 & 235 & 8.5 & 97 & 2.2 & 0 & 0.0 & 75 & 0.6 & 29 & 7.6 & 446 & 2.1 \\
\hline 35-39 & 2 & 1.4 & 5 & 0.4 & 336 & 12.9 & 135 & 3.2 & 1 & 2.4 & 118 & 1.0 & 34 & 10.5 & 631 & 3.1 \\
\hline 40-44 & 5 & 3.5 & 10 & 0.7 & 464 & 18.2 & 193 & 4.9 & 1 & 2.7 & 243 & 2.0 & 60 & 21.5 & 976 & 4.8 \\
\hline $45-49$ & 8 & 5.4 & 9 & 0.7 & 682 & 26.4 & 315 & 9.1 & 0 & 0.0 & 489 & 3.7 & 99 & 39.9 & 1,602 & 7.7 \\
\hline 50-54 & 10 & 6.2 & 11 & 1.0 & 999 & 36.9 & 403 & 13.3 & 1 & 2.9 & 842 & 5.6 & 156 & 64.7 & 2,422 & 10.9 \\
\hline 55-59 & 8 & 5.2 & 7 & 0.7 & 980 & 38.4 & 331 & 13.6 & 1 & 3.3 & 796 & 5.2 & 163 & 74.6 & 2,286 & 10.5 \\
\hline $60-64$ & 4 & 3.2 & 4 & 0.5 & 789 & 38.3 & 249 & 13.6 & 0 & 0.0 & 518 & 3.7 & 134 & 78.2 & 1,698 & 8.9 \\
\hline$\geq 65$ & 6 & 2.4 & 9 & 0.5 & 856 & 20.4 & 300 & 8.0 & 0 & 0.0 & 740 & 2.0 & 114 & 33.2 & 2,025 & 4.2 \\
\hline \multicolumn{17}{|l|}{ Transmission category ${ }^{d}$} \\
\hline \multicolumn{17}{|l|}{ Male adult or adolescent } \\
\hline Male-to-male sexual contact & 16 & - & 43 & - & 1,640 & - & 878 & - & 2 & - & 2,329 & - & 271 & - & 5,180 & - \\
\hline Injection drug use & 4 & - & 3 & - & 950 & - & 411 & - & 0 & - & 348 & - & 153 & - & 1,869 & - \\
\hline $\begin{array}{l}\text { Male-to-male sexual contact and } \\
\text { injection drug use }\end{array}$ & 7 & - & 3 & - & 349 & - & 200 & - & 0 & - & 443 & - & 122 & - & 1,124 & - \\
\hline Heterosexual contact ${ }^{e}$ & 5 & - & 7 & - & 764 & - & 183 & - & 2 & - & 171 & - & 59 & - & 1,192 & - \\
\hline Perinatal & 0 & - & 1 & - & 14 & - & 5 & - & 0 & - & 3 & - & 3 & - & 26 & - \\
\hline Other $^{f}$ & 0 & - & 0 & - & 15 & - & 7 & - & 0 & - & 32 & - & 7 & - & 62 & - \\
\hline Subtotal & 33 & 3.5 & 57 & 0.8 & 3,734 & 24.3 & 1,684 & 7.8 & 4 & 1.8 & 3,326 & 4.0 & 615 & 30.0 & 9,453 & 7.2 \\
\hline
\end{tabular}


Table 19a. Deaths of persons with diagnosed HIV infection ever classified as stage 3 (AIDS), by race/ethnicity and selected characteristics, 2015-United States (cont)



\section{Female adult or adolescent}

Injection drug use

Heterosexual contact ${ }^{\mathrm{e}}$

Perinatal

Other ${ }^{f}$

Subtotal

$\begin{array}{rrr}8 & - & 0 \\ 8 & - & 5 \\ 0 & - & 0 \\ 1 & - & 0 \\ 16 & 1.6 & 5\end{array}$

$\begin{array}{rrrrrr}- & 539 & - & 182 & - & 0 \\ - & 1,271 & - & 223 & - & 0 \\ - & 31 & - & 7 & - & 0 \\ - & 9 & - & 4 & - & 0 \\ 0.1 & 1,850 & 10.7 & 416 & 2.0 & 0\end{array}$

$$
\begin{aligned}
& - \\
& - \\
& -
\end{aligned}
$$

$\begin{array}{rrrrrr}265 & - & 97 & - & 1,090 & - \\ 276 & - & 102 & - & 1,885 & - \\ 6 & - & 1 & - & 45 & - \\ 6 & - & 2 & - & 22 & - \\ 553 & 0.6 & 202 & 9.2 & 3,042 & 2.2\end{array}$

\section{Child ( $<13$ yrs at death)}

Perinatal

Other ${ }^{\mathrm{f}}$

Subtotal

$\begin{array}{cc}0 & - \\ 0 & - \\ 0 & 0.0\end{array}$

$\stackrel{\bullet}{\underline{0}}$

Region of residence ${ }^{9}$

Northeast

Midwest

South

West

Total

$\begin{array}{rrrrrrrrrrrrrrrr}0 & 0.0 & 8 & 0.2 & 1,183 & 18.7 & 767 & 9.7 & 0 & 0.0 & 624 & 1.7 & 301 & 33.2 & 2,883 & 5.1 \\ 8 & 2.0 & 4 & 0.2 & 617 & 8.8 & 101 & 2.0 & 0 & 0.0 & 557 & 1.1 & 78 & 6.2 & 1,365 & 2.0 \\ 20 & 2.5 & 11 & 0.3 & 3,405 & 14.8 & 722 & 3.5 & 0 & 0.0 & 1,690 & 2.4 & 311 & 14.4 & 6,159 & 5.1 \\ 21 & 2.0 & 39 & 0.5 & 381 & 11.1 & 510 & 2.3 & 4 & 1.0 & 1,008 & 2.6 & 127 & 5.6 & 2,090 & 2.8 \\ \mathbf{4 9} & \mathbf{2 . 1} & \mathbf{6 2} & \mathbf{0 . 4} & \mathbf{5 , 5 8 6} & \mathbf{1 4 . 0} & \mathbf{2 , 1 0 0} & \mathbf{3 . 7} & \mathbf{4} & \mathbf{0 . 7} & \mathbf{3 , 8 7 9} & \mathbf{2 . 0} & \mathbf{8 1 7} & \mathbf{1 2 . 4} & \mathbf{1 2 , 4 9 7} & \mathbf{3 . 9}\end{array}$

Note. Deaths of persons with a diagnosis of HIV infection may be due to any cause. Numbers less than 12 , and rates based on these numbers, should be interpreted with caution

${ }^{a}$ Includes Asian/Pacific Islander legacy cases (see Technical Notes).

${ }^{b}$ Hispanics/Latinos can be of any race.

${ }^{c}$ Rates are per 100,000 population. Rates are not calculated by transmission category because of the lack of denominator data.

${ }^{d}$ Data have been statistically adjusted to account for missing transmission category; therefore, values may not sum to column subtotals and total.

e Heterosexual contact with a person known to have, or to be at high risk for, HIV infection

f Includes hemophilia, blood transfusion, and risk factor not reported or not identified.

${ }^{9}$ Data are based on residence at death. When information on residence at death was not available, state at death (where a person's death occurred) was used. 


\begin{tabular}{|c|c|c|c|c|c|c|c|c|}
\hline & $\begin{array}{c}\text { American Indian/ } \\
\text { Alaska Native } \\
\text { No. }\end{array}$ & $\begin{array}{c}\text { Asian }^{a} \\
\text { No. }\end{array}$ & $\begin{array}{c}\text { Black/ } \\
\text { African American } \\
\text { No. }\end{array}$ & $\begin{array}{c}\text { Hispanic/Latino }^{b} \\
\text { No. }\end{array}$ & $\begin{array}{c}\text { Native Hawaiian/ } \\
\text { Other Pacific } \\
\text { Islander } \\
\text { No. }\end{array}$ & $\begin{array}{l}\text { White } \\
\text { No. }\end{array}$ & $\begin{array}{c}\text { Multiple races } \\
\text { No. }\end{array}$ & $\begin{array}{l}\text { Total } \\
\text { No. }\end{array}$ \\
\hline \multicolumn{9}{|l|}{ Age at death (yr) } \\
\hline$<13$ & 0 & 0 & 2 & 0 & 0 & 0 & 0 & 2 \\
\hline 13-14 & 0 & 0 & 0 & 0 & 0 & 0 & 0 & 0 \\
\hline 15-19 & 0 & 0 & 4 & 0 & 0 & 3 & 0 & 7 \\
\hline $20-24$ & 0 & 0 & 75 & 16 & 0 & 11 & 6 & 108 \\
\hline $25-29$ & 1 & 2 & 164 & 65 & 0 & 44 & 22 & 298 \\
\hline 30-34 & 5 & 5 & 235 & 113 & 0 & 75 & 29 & 462 \\
\hline $35-39$ & 2 & 5 & 336 & 147 & 1 & 118 & 35 & 644 \\
\hline 40-44 & 5 & 10 & 464 & 217 & 1 & 243 & 60 & 1,000 \\
\hline $45-49$ & 8 & 9 & 682 & 352 & 0 & 490 & 99 & 1,640 \\
\hline $50-54$ & 10 & 11 & 1,000 & 469 & 2 & 842 & 156 & 2,490 \\
\hline $55-59$ & 8 & 7 & 980 & 376 & 1 & 796 & 163 & 2,331 \\
\hline $60-64$ & 4 & 4 & 789 & 286 & 0 & 518 & 134 & 1,735 \\
\hline$\geq 65$ & 6 & 9 & 858 & 343 & 0 & 740 & 114 & 2,070 \\
\hline \multicolumn{9}{|l|}{ Transmission category $^{\mathrm{C}}$} \\
\hline \multicolumn{9}{|l|}{ Male adult or adolescent } \\
\hline Male-to-male sexual contact & 16 & 43 & 1,641 & 929 & 3 & 2,330 & 272 & 5,234 \\
\hline Injection drug use & 4 & 3 & 951 & 504 & 0 & 348 & 153 & 1,963 \\
\hline Male-to-male sexual contact and injection drug use & 7 & 3 & 349 & 225 & 0 & 443 & 122 & 1,149 \\
\hline Heterosexual contact ${ }^{d}$ & 5 & 7 & 765 & 225 & 2 & 171 & 59 & 1,235 \\
\hline Perinatal & 0 & 1 & 14 & 6 & 0 & 3 & 3 & 27 \\
\hline Other ${ }^{\mathrm{e}}$ & 0 & 0 & 15 & 7 & 0 & 32 & 7 & 62 \\
\hline Subtotal & 33 & 57 & 3,735 & 1,897 & 5 & 3,327 & 616 & 9,670 \\
\hline
\end{tabular}


Table 19b. Deaths of persons with diagnosed HIV infection ever classified as stage 3 (AIDS), by race/ethnicity and selected characteristics, 2015-United States and 6 dependent areas (cont)



\section{Female adult or adolescent}

Injection drug use

Heterosexual contact ${ }^{d}$

Perinatal

Other ${ }^{\mathrm{e}}$

Subtotal

8
8
0
1
16

$\begin{array}{rr}0 & 539 \\ 5 & 1,273 \\ 0 & 31 \\ 0 & 9 \\ 5 & 1,852\end{array}$

$\begin{array}{rl} & \\ 195 & 0 \\ 280 & 0 \\ 8 & 0 \\ 4 & 0 \\ 487 & 0\end{array}$

\section{Child ( $<13$ yrs at end of year)}

Perinatal

Other ${ }^{\mathrm{e}}$

Subtotal

$\begin{array}{ll}0 & 0 \\ 0 & 0 \\ 0 & 0\end{array}$

$\stackrel{\varrho}{\mathscr{C}}$

\section{Region of residence ${ }^{f}$}

Northeast

Midwest

South

West

U.S. dependent areas

0

8

20

21

Total

$49 \quad 62$

$\begin{array}{rrrr}8 & 1,183 & 767 & 0 \\ 4 & 617 & 101 & 0 \\ 11 & 3,405 & 722 & 0 \\ 39 & 381 & 510 & 4 \\ 0 & 3 & 284 & 1 \\ \mathbf{6 2} & \mathbf{5 , 5 8 9} & \mathbf{2 , 3 8 4} & \mathbf{5}\end{array}$

265
276
6
6
553

97

1,103

(n)

$\begin{array}{ll}0 & 0 \\ 0 & 0 \\ 0 & 0\end{array}$

0
0
0

2
0
2

Note. Deaths of persons with a diagnosis of HIV infection may be due to any cause. Numbers less than 12 should be interpreted with caution.

a Includes Asian/Pacific Islander legacy cases (see Technical Notes).

${ }^{b}$ Hispanics/Latinos can be of any race.

${ }^{\mathrm{C}}$ Data have been statistically adjusted to account for missing transmission category; therefore, values may not sum to column subtotals and total.

d Heterosexual contact with a person known to have, or to be at high risk for, HIV infection.

e Includes hemophilia, blood transfusion, and risk factor not reported or not identified.

${ }^{f}$ Data are based on residence at death. When information on residence at death was not available, state at death (where a person's death occurred) was used. 


\begin{tabular}{|c|c|c|c|c|c|c|c|c|c|c|}
\hline & \multicolumn{2}{|c|}{2011} & \multicolumn{2}{|c|}{2012} & \multicolumn{2}{|c|}{2013} & \multicolumn{2}{|c|}{2014} & \multicolumn{2}{|c|}{2015} \\
\hline & No. & Rate $^{a}$ & No. & Rate $^{a}$ & No. & Rate $^{a}$ & No. & Rate $^{a}$ & No. & Rate $^{a}$ \\
\hline \multicolumn{11}{|l|}{ Age at end of year (yr) } \\
\hline$<13$ & 2,808 & 5.3 & 2,713 & 5.1 & 2,589 & 4.9 & 2,465 & 4.7 & 2,322 & 4.4 \\
\hline $13-14$ & 1,065 & 12.9 & 894 & 10.8 & 780 & 9.3 & 746 & 8.9 & 721 & 8.7 \\
\hline 15-19 & 6,813 & 31.4 & 6,300 & 29.5 & 5,703 & 26.9 & 5,305 & 25.2 & 4,959 & 23.5 \\
\hline 20-24 & 30,663 & 138.3 & 31,896 & 141.1 & 32,056 & 140.4 & 31,723 & 138.6 & 30,812 & 135.8 \\
\hline 25-29 & 51,527 & 242.0 & 53,945 & 252.2 & 57,405 & 266.1 & 61,540 & 280.3 & 65,540 & 292.6 \\
\hline 30-34 & 69,908 & 340.7 & 71,675 & 342.7 & 72,943 & 342.9 & 74,731 & 347.5 & 76,656 & 354.6 \\
\hline 35-39 & 85,520 & 436.2 & 84,090 & 431.2 & 84,290 & 429.6 & 85,542 & 430.1 & 88,220 & 434.3 \\
\hline 40-44 & 129,318 & 614.4 & 123,550 & 587.2 & 116,978 & 560.6 & 110,690 & 538.4 & 103,583 & 513.9 \\
\hline $45-49$ & 167,492 & 755.4 & 164,020 & 755.7 & 158,609 & 747.1 & 151,482 & 726.4 & 146,349 & 703.6 \\
\hline $50-54$ & 142,621 & 631.8 & 151,584 & 671.1 & 160,339 & 710.4 & 167,641 & 743.7 & 171,172 & 767.9 \\
\hline $55-59$ & 95,194 & 469.8 & 104,566 & 503.2 & 113,356 & 534.9 & 121,961 & 567.6 & 131,430 & 603.8 \\
\hline 60-64 & 52,359 & 293.9 & 58,976 & 330.9 & 65,910 & 363.7 & 73,780 & 397.8 & 81,438 & 427.8 \\
\hline$\geq 65$ & 38,978 & 94.2 & 45,483 & 105.4 & 52,819 & 118.2 & 60,888 & 131.8 & 70,644 & 148.0 \\
\hline \multicolumn{11}{|l|}{ Race/ethnicity } \\
\hline American Indian/Alaska Native & 2,424 & 105.9 & 2,546 & 110.2 & 2,636 & 113.1 & 2,763 & 117.5 & 2,904 & 122.6 \\
\hline Asian ${ }^{b}$ & 9,508 & 62.5 & 10,250 & 65.3 & 11,028 & 68.2 & 11,945 & 71.6 & 12,887 & 74.8 \\
\hline Black/African American & 363,650 & 947.2 & 374,384 & 965.5 & 384,587 & 982.7 & 394,977 & 999.7 & 405,857 & $1,017.8$ \\
\hline Hispanic/Latinoc & 185,278 & 356.9 & 192,350 & 363.0 & 198,971 & 368.0 & 206,185 & 373.6 & 213,736 & 379.4 \\
\hline Native Hawaiian/Other Pacific Islander & 693 & 135.7 & 740 & 141.9 & 779 & 146.1 & 816 & 149.9 & 891 & 160.3 \\
\hline White & 275,524 & 139.5 & 281,514 & 142.4 & 287,329 & 145.3 & 293,023 & 148.1 & 298,670 & 150.9 \\
\hline Multiple races & 36,187 & 621.1 & 36,928 & 614.6 & 37,471 & 605.1 & 37,813 & 592.6 & 37,934 & 577.3 \\
\hline \multicolumn{11}{|l|}{ Transmission category $^{d}$} \\
\hline \multicolumn{11}{|l|}{ Male adult or adolescent } \\
\hline Male-to-male sexual contact & 446,305 & - & 466,328 & - & 485,717 & - & 506,037 & - & 526,456 & - \\
\hline Injection drug use & 79,507 & - & 78,524 & - & 77,526 & - & 76,408 & - & 75,569 & - \\
\hline $\begin{array}{l}\text { Male-to-male sexual contact and } \\
\text { injection drug use }\end{array}$ & 53,049 & - & 53,194 & - & 53,181 & - & 53,144 & - & 53,090 & - \\
\hline Heterosexual contact ${ }^{\mathrm{e}}$ & 69,005 & - & 70,866 & - & 72,605 & - & 74,296 & - & 76,038 & - \\
\hline Perinatal & 4,143 & - & 4,276 & - & 4,390 & - & 4,518 & - & 4,621 & - \\
\hline Other $^{f}$ & 3,094 & - & 3,078 & - & 3,056 & - & 3,052 & - & 3,058 & - \\
\hline Subtotal & 655,103 & 519.0 & 676,266 & 530.5 & 696,475 & 541.3 & 717,455 & 552.4 & 738,832 & 563.9 \\
\hline
\end{tabular}









\begin{tabular}{|c|c|c|c|c|c|c|c|c|c|c|}
\hline & \multicolumn{2}{|c|}{2011} & \multicolumn{2}{|c|}{2012} & \multicolumn{2}{|c|}{2013} & \multicolumn{2}{|c|}{2014} & \multicolumn{2}{|c|}{2015} \\
\hline & No. & Rate $^{a}$ & No. & Rate $^{a}$ & No. & Rate $^{a}$ & No. & Rate $^{a}$ & No. & Rate $^{a}$ \\
\hline \multicolumn{11}{|l|}{ Age at end of year $(y r)$} \\
\hline$<13$ & 2,847 & 5.3 & 2,738 & 5.1 & 2,608 & 4.9 & 2,482 & 4.7 & 2,334 & 4.4 \\
\hline $13-14$ & 1,082 & 12.9 & 916 & 10.9 & 801 & 9.4 & 754 & 8.9 & 727 & 8.7 \\
\hline $15-19$ & 6,943 & 31.6 & 6,414 & 29.6 & 5,790 & 27.0 & 5,394 & 25.3 & 5,026 & 23.5 \\
\hline $20-24$ & 31,066 & 138.3 & 32,323 & 141.2 & 32,496 & 140.6 & 32,152 & 138.7 & 31,208 & 135.8 \\
\hline $25-29$ & 52,266 & 242.6 & 54,669 & 252.6 & 58,105 & 266.2 & 62,288 & 280.5 & 66,324 & 292.8 \\
\hline $30-34$ & 71,208 & 342.5 & 72,892 & 344.2 & 74,117 & 344.3 & 75,802 & 348.5 & 77,627 & 355.2 \\
\hline $35-39$ & 87,231 & 439.0 & 85,713 & 433.8 & 85,854 & 432.0 & 87,052 & 432.3 & 89,729 & 436.5 \\
\hline $40-44$ & 131,837 & 618.6 & 125,905 & 591.0 & 119,119 & 563.9 & 112,718 & 541.6 & 105,428 & 516.7 \\
\hline $45-49$ & 170,769 & 760.7 & 167,188 & 760.8 & 161,657 & 752.1 & 154,317 & 730.9 & 149,074 & 708.1 \\
\hline $50-54$ & 145,469 & 637.0 & 154,565 & 676.4 & 163,458 & 715.9 & 170,852 & 749.2 & 174,372 & 773.3 \\
\hline $55-59$ & 97,150 & 473.7 & 106,656 & 507.2 & 115,658 & 539.4 & 124,374 & 572.2 & 133,984 & 608.6 \\
\hline $60-64$ & 53,512 & 296.4 & 60,237 & 333.7 & 67,244 & 366.4 & 75,268 & 400.9 & 83,068 & 431.2 \\
\hline$\geq 65$ & 40,059 & 95.5 & 46,716 & 106.7 & 54,207 & 119.6 & 62,414 & 133.2 & 72,388 & 149.5 \\
\hline \multicolumn{11}{|l|}{ Race/ethnicity } \\
\hline American Indian/Alaska Native & 2,425 & - & 2,547 & - & 2,637 & - & 2,764 & - & 2,905 & - \\
\hline Asian ${ }^{b}$ & 9,548 & - & 10,291 & - & 11,069 & - & 11,985 & - & 12,932 & - \\
\hline Black/African American & 363,970 & - & 374,696 & - & 384,906 & - & 395,300 & - & 406,175 & - \\
\hline Hispanic/Latinoc & 201,990 & - & 209,143 & - & 215,852 & - & 223,089 & - & 230,701 & - \\
\hline Native Hawaiian/Other Pacific Islander & 711 & - & 759 & - & 797 & - & 837 & - & 917 & - \\
\hline White & 275,592 & - & 281,574 & - & 287,395 & - & 293,096 & - & 298,744 & - \\
\hline Multiple races & 36,198 & - & 36,939 & - & 37,479 & - & 37,821 & - & 37,945 & - \\
\hline \multicolumn{11}{|l|}{$\begin{array}{l}\text { Transmission category } \\
\text { Male adult or adolescent }\end{array}$} \\
\hline Male-to-male sexual contact & 450,092 & - & 470,301 & - & 489,887 & - & 510,403 & - & 530,970 & - \\
\hline Injection drug use & 83,936 & - & 82,827 & - & 81,725 & - & 80,502 & - & 79,599 & - \\
\hline $\begin{array}{l}\text { Male-to-male sexual contact and } \\
\text { injection drug use }\end{array}$ & 53,929 & - & 54,045 & - & 54,019 & - & 53,951 & - & 53,898 & 一 \\
\hline Heterosexual contact ${ }^{\mathrm{e}}$ & 71,456 & - & 73,354 & - & 75,119 & - & 76,839 & - & 78,612 & - \\
\hline Perinatal & 4,267 & - & 4,404 & - & 4,518 & - & 4,642 & - & 4,744 & 一 \\
\hline Other $^{f}$ & 3,135 & - & 3,118 & - & 3,097 & - & 3,093 & - & 3,098 & 一 \\
\hline Subtotal & 666,815 & 521.6 & 688,049 & 533.1 & 708,365 & 543.8 & 729,430 & 554.9 & 750,921 & 566.4 \\
\hline
\end{tabular}




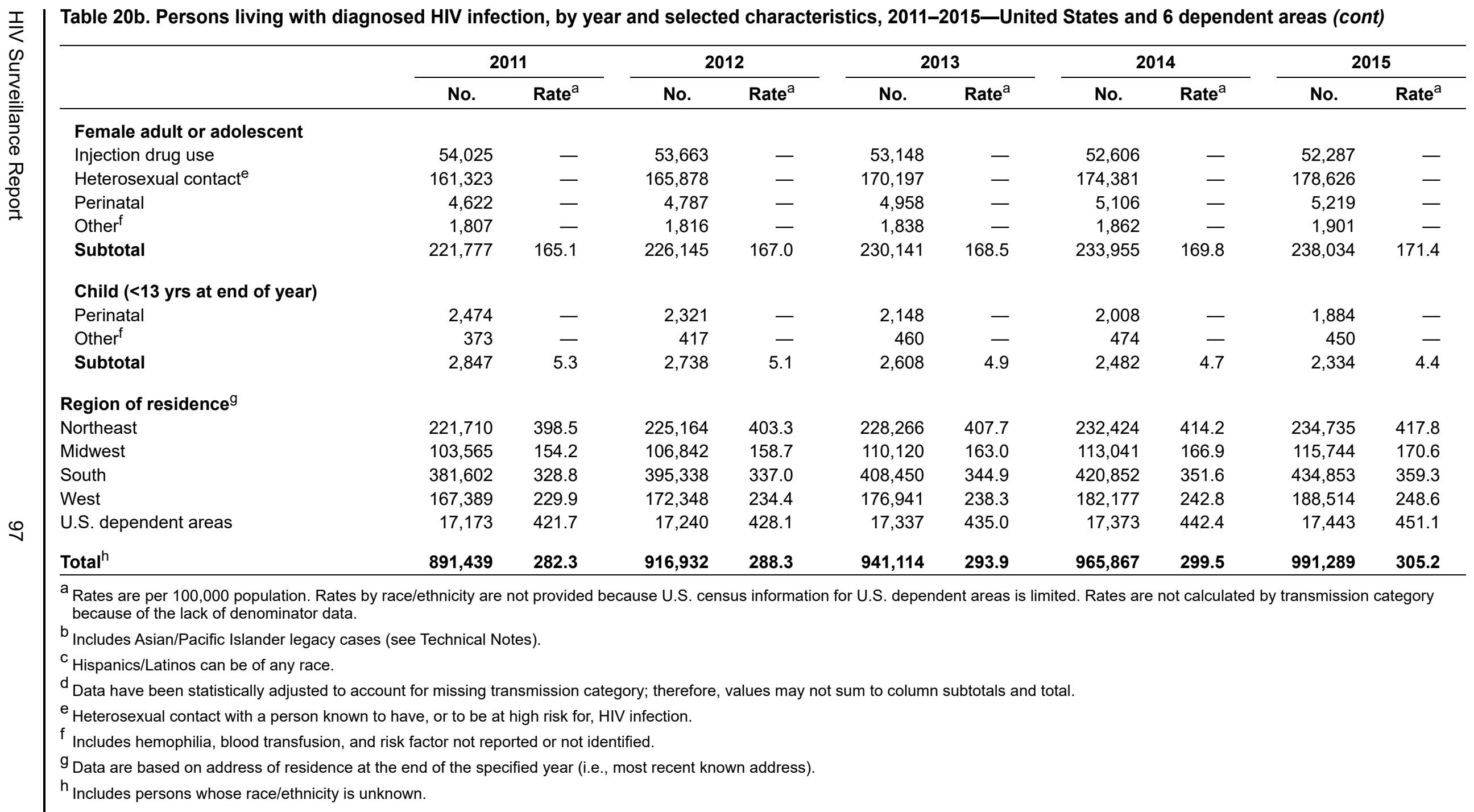




\begin{tabular}{|c|c|c|c|c|c|c|c|c|c|c|}
\hline & \multicolumn{2}{|c|}{2011} & \multicolumn{2}{|c|}{2012} & \multicolumn{2}{|c|}{2013} & \multicolumn{2}{|c|}{2014} & \multicolumn{2}{|c|}{2015} \\
\hline & No. & Rate $^{a}$ & No. & Rate $^{a}$ & No. & Rate $^{a}$ & No. & Rate $^{a}$ & No. & Rate $^{2}$ \\
\hline \multicolumn{11}{|l|}{ Age at end of year (yr) } \\
\hline$<13$ & 380 & 0.7 & 323 & 0.6 & 280 & 0.5 & 300 & 0.6 & 276 & 0.5 \\
\hline $13-14$ & 255 & 3.1 & 194 & 2.3 & 154 & 1.8 & 134 & 1.6 & 132 & 1.6 \\
\hline $15-19$ & 2,280 & 10.5 & 1,965 & 9.2 & 1,702 & 8 & 1,305 & 6.2 & 972 & 4.6 \\
\hline $20-24$ & 7,997 & 36.1 & 8,251 & 36.5 & 8,261 & 36.2 & 7,519 & 32.8 & 6,736 & 29.7 \\
\hline $25-29$ & 16,164 & 75.9 & 16,541 & 77.3 & 17,393 & 80.6 & 17,876 & 81.4 & 18,017 & 80.4 \\
\hline $30-34$ & 27,992 & 136.4 & 28,294 & 135.3 & 28,207 & 132.6 & 27,572 & 128.2 & 27,258 & 126.1 \\
\hline $35-39$ & 41,870 & 213.5 & 40,401 & 207.1 & 39,758 & 202.6 & 38,920 & 195.7 & 38,606 & 190.1 \\
\hline $40-44$ & 72,482 & 344.4 & 68,272 & 324.5 & 63,609 & 304.9 & 58,941 & 286.7 & 53,713 & 266.5 \\
\hline $45-49$ & 102,825 & 463.7 & 99,837 & 460.0 & 95,450 & 449.6 & 89,572 & 429.5 & 84,744 & 407.4 \\
\hline $50-54$ & 91,647 & 406.0 & 96,909 & 429.0 & 101,917 & 451.6 & 105,686 & 468.8 & 106,763 & 479.0 \\
\hline $55-59$ & 62,700 & 309.4 & 68,768 & 330.9 & 74,289 & 350.5 & 79,162 & 368.4 & 84,701 & 389.1 \\
\hline $60-64$ & 34,669 & 194.6 & 39,001 & 218.9 & 43,635 & 240.8 & 48,747 & 262.8 & 53,541 & 281.2 \\
\hline$\geq 65$ & 26,142 & 63.2 & 30,425 & 70.5 & 35,222 & 78.8 & 40,485 & 87.6 & 46,824 & 98.1 \\
\hline \multicolumn{11}{|l|}{ Race/ethnicity } \\
\hline American Indian/Alaska Native & 1,321 & 57.7 & 1,371 & 59.3 & 1,404 & 60.2 & 1,448 & 61.6 & 1,492 & 63.0 \\
\hline Asian ${ }^{b}$ & 5,081 & 33.4 & 5,381 & 34.3 & 5,673 & 35.1 & 5,947 & 35.6 & 6,211 & 36.1 \\
\hline Black/African American & 197,850 & 515.3 & 203,595 & 525.1 & 208,803 & 533.5 & 211,928 & 536.4 & 215,024 & 539.2 \\
\hline Hispanic/Latinoc & 110,808 & 213.5 & 114,036 & 215.2 & 116,885 & 216.2 & 118,803 & 215.3 & 120,770 & 214.4 \\
\hline Native Hawaiian/Other Pacific Islander & 388 & 76.0 & 406 & 77.8 & 420 & 78.8 & 432 & 79.4 & 452 & 81.3 \\
\hline White & 150,334 & 76.1 & 152,361 & 77.1 & 154,318 & 78.0 & 155,200 & 78.4 & 155,958 & 78.8 \\
\hline Multiple races & 21,574 & 370.3 & 21,985 & 365.9 & 22,329 & 360.6 & 22,416 & 351.3 & 22,331 & 339.9 \\
\hline \multicolumn{11}{|l|}{ Transmission category ${ }^{d}$} \\
\hline \multicolumn{11}{|l|}{ Male adult or adolescent } \\
\hline Male-to-male sexual contact & 237,904 & - & 245,948 & - & 253,709 & - & 258,848 & - & 263,791 & - \\
\hline Injection drug use & 53,043 & - & 52,527 & - & 51,860 & - & 51,001 & - & 50,139 & - \\
\hline $\begin{array}{l}\text { Male-to-male sexual contact and } \\
\text { injection drug use }\end{array}$ & 34,348 & - & 34,434 & - & 34,320 & - & 33,965 & - & 33,617 & - \\
\hline Heterosexual contact ${ }^{\mathrm{e}}$ & 41,892 & - & 43,251 & - & 44,511 & - & 45,396 & - & 46,187 & - \\
\hline Perinatal & 2,298 & - & 2,343 & - & 2,405 & - & 2,446 & - & 2,513 & - \\
\hline Other ${ }^{f}$ & 2,103 & - & 2,094 & - & 2,094 & - & 2,082 & - & 2,053 & - \\
\hline Subtotal & 371,590 & 294.4 & 380,598 & 298.6 & 388,899 & 302.2 & 393,739 & 303.1 & 398,300 & 304.0 \\
\hline
\end{tabular}




\begin{tabular}{|c|c|c|c|c|c|c|c|c|c|c|}
\hline & \multicolumn{2}{|c|}{2011} & \multicolumn{2}{|c|}{2012} & \multicolumn{2}{|c|}{2013} & \multicolumn{2}{|c|}{2014} & \multicolumn{2}{|c|}{2015} \\
\hline & No. & Rate $^{a}$ & No. & Rate $^{a}$ & No. & Rate $^{a}$ & No. & Rate $^{a}$ & No. & Rate $^{\mathrm{a}}$ \\
\hline \multicolumn{11}{|c|}{ Female adult or adolescent } \\
\hline Injection drug use & 32,011 & - & 31,957 & - & 31,721 & - & 31,367 & - & 31,072 & - \\
\hline Heterosexual contact $\mathrm{e}^{\mathrm{e}}$ & 79,857 & - & 82,668 & - & 85,232 & - & 87,002 & - & 88,777 & - \\
\hline Perinatal & 2,466 & - & 2,538 & - & 2,650 & - & 2,710 & - & 2,759 & - \\
\hline Other ${ }^{f}$ & 1,099 & - & 1,097 & - & 1,095 & - & 1,100 & - & 1,099 & - \\
\hline Subtotal & 115,433 & 87.1 & 118,260 & 88.5 & 120,698 & 89.5 & 122,180 & 89.8 & 123,707 & 90.2 \\
\hline \multicolumn{11}{|c|}{ Child ( $<13$ yrs at end of year) } \\
\hline Perinatal & 358 & - & 302 & - & 259 & - & 269 & - & 243 & - \\
\hline Other ${ }^{f}$ & 22 & - & 21 & - & 21 & - & 31 & - & 33 & - \\
\hline Subtotal & 380 & 0.7 & 323 & 0.6 & 280 & 0.5 & 300 & 0.6 & 276 & 0.5 \\
\hline \multicolumn{11}{|l|}{ Region of residence ${ }^{g}$} \\
\hline Northeast & 131,321 & 236.0 & 132,481 & 237.3 & 133,396 & 238.3 & 134,082 & 238.9 & 133,827 & 238.2 \\
\hline Midwest & 54,467 & 81.1 & 55,857 & 83.0 & 57,199 & 84.7 & 58,110 & 85.8 & 58,655 & 86.5 \\
\hline South & 204,202 & 175.9 & 211,541 & 180.3 & 218,324 & 184.4 & 222,083 & 185.5 & 226,408 & 187.1 \\
\hline West & 97,413 & 133.8 & 99,302 & 135.0 & 100,958 & 136.0 & 101,944 & 135.9 & 103,393 & 136.3 \\
\hline Total $^{\mathrm{h}}$ & 487,403 & 156.4 & 499,181 & 159.0 & 509,877 & 161.2 & 516,219 & 162.0 & 522,283 & 162.8 \\
\hline  & $\begin{array}{l}\text { Technical } \mathrm{N} \\
\text { r missing tr } \\
\text { al or to be a } \\
\text { d of the spe }\end{array}$ & $\begin{array}{l}\text { ansmissic } \\
\text { s). } \\
\text { mission } \\
\text { gh risk fo } \\
\text { ed or not } \\
\text { ed year ( }\end{array}$ & $\begin{array}{l}\text { y; therefore } \\
\text { fection. } \\
\text { ed. } \\
\text { st recent kn }\end{array}$ & f the lack & ominator da & ubtotals a & & & & \\
\hline
\end{tabular}




\begin{tabular}{|c|c|c|c|c|c|c|c|c|c|c|}
\hline & \multicolumn{2}{|c|}{2011} & \multicolumn{2}{|c|}{2012} & \multicolumn{2}{|c|}{2013} & \multicolumn{2}{|c|}{2014} & \multicolumn{2}{|c|}{2015} \\
\hline & No. & Rate $^{a}$ & No. & Rate $^{a}$ & No. & Rate $^{a}$ & No. & Rate $^{a}$ & No. & Rate $^{a}$ \\
\hline \multicolumn{11}{|l|}{ Age at end of year (yr) } \\
\hline$<13$ & 386 & 0.7 & 328 & 0.6 & 283 & 0.5 & 303 & 0.6 & 278 & 0.5 \\
\hline $13-14$ & 261 & 3.1 & 198 & 2.4 & 159 & 1.9 & 135 & 1.6 & 133 & 1.6 \\
\hline 15-19 & 2,329 & 10.6 & 2,009 & 9.3 & 1,738 & 8.1 & 1,335 & 6.3 & 991 & 4.6 \\
\hline $20-24$ & 8,114 & 36.1 & 8,363 & 36.5 & 8,372 & 36.2 & 7,619 & 32.9 & 6,827 & 29.7 \\
\hline 25-29 & 16,370 & 76.0 & 16,732 & 77.3 & 17,585 & 80.6 & 18,077 & 81.4 & 18,216 & 80.4 \\
\hline 30-34 & 28,390 & 136.6 & 28,659 & 135.3 & 28,553 & 132.6 & 27,888 & 128.2 & 27,536 & 126.0 \\
\hline $35-39$ & 42,603 & 214.4 & 41,083 & 207.9 & 40,387 & 203.2 & 39,503 & 196.2 & 39,144 & 190.4 \\
\hline 40-44 & 73,790 & 346.2 & 69,461 & 326.1 & 64,689 & 306.2 & 59,941 & 288.0 & 54,618 & 267.7 \\
\hline $45-49$ & 104,878 & 467.2 & 101,774 & 463.1 & 97,259 & 452.5 & 91,196 & 431.9 & 86,258 & 409.7 \\
\hline $50-54$ & 93,474 & 409.3 & 98,805 & 432.4 & 103,935 & 455.2 & 107,755 & 472.5 & 108,778 & 482.4 \\
\hline $55-59$ & 64,015 & 312.1 & 70,183 & 333.8 & 75,840 & 353.7 & 80,763 & 371.6 & 86,403 & 392.5 \\
\hline $60-64$ & 35,469 & 196.5 & 39,875 & 220.9 & 44,552 & 242.8 & 49,780 & 265.1 & 54,647 & 283.6 \\
\hline$\geq 65$ & 26,874 & 64.0 & 31,264 & 71.4 & 36,173 & 79.8 & 41,523 & 88.6 & 48,013 & 99.2 \\
\hline \multicolumn{11}{|l|}{ Race/ethnicity } \\
\hline American Indian/Alaska Native & 1,322 & - & 1,372 & - & 1,405 & - & 1,449 & - & 1,493 & - \\
\hline Asian ${ }^{b}$ & 5,098 & - & 5,397 & - & 5,689 & - & 5,963 & - & 6,228 & - \\
\hline Black/African American & 198,020 & - & 203,764 & - & 208,973 & - & 212,097 & - & 215,195 & - \\
\hline Hispanic/Latino ${ }^{C}$ & 120,118 & - & 123,354 & - & 126,296 & - & 128,164 & - & 130,085 & - \\
\hline Native Hawaiian/Other Pacific Islander & 400 & - & 419 & - & 431 & - & 443 & - & 466 & - \\
\hline White & 150,370 & - & 152,394 & - & 154,355 & - & 155,239 & - & 155,996 & - \\
\hline Multiple races & 21,578 & - & 21,988 & - & 22,331 & - & 22,418 & - & 22,334 & - \\
\hline \multicolumn{11}{|l|}{ Transmission category $^{d}$} \\
\hline \multicolumn{11}{|l|}{ Male adult or adolescent } \\
\hline Male-to-male sexual contact & 239,905 & - & 248,006 & - & 255,845 & - & 261,019 & - & 265,977 & - \\
\hline Injection drug use & 55,644 & - & 55,074 & - & 54,371 & - & 53,456 & - & 52,562 & - \\
\hline $\begin{array}{l}\text { Male-to-male sexual contact and } \\
\text { injection drug use }\end{array}$ & 34,916 & - & 34,989 & - & 34,863 & - & 34,492 & - & 34,141 & - \\
\hline Heterosexual contact ${ }^{\mathrm{e}}$ & 43,288 & - & 44,658 & - & 45,943 & - & 46,847 & - & 47,653 & - \\
\hline Perinatal & 2,375 & - & 2,421 & - & 2,485 & - & 2,524 & - & 2,589 & - \\
\hline Other $^{f}$ & 2,127 & - & 2,115 & - & 2,116 & - & 2,102 & - & 2,073 & - \\
\hline Subtotal & 378,256 & 295.9 & 387,263 & 300.0 & 395,624 & 303.7 & 400,441 & 304.6 & 404,995 & 305.5 \\
\hline
\end{tabular}




\begin{tabular}{|c|c|c|c|c|c|c|c|c|c|c|}
\hline & \multicolumn{2}{|c|}{2011} & \multicolumn{2}{|c|}{2012} & \multicolumn{2}{|c|}{2013} & \multicolumn{2}{|c|}{2014} & \multicolumn{2}{|c|}{2015} \\
\hline & No. & Rate $^{a}$ & No. & Rate $^{a}$ & No. & Rate $^{a}$ & No. & Rate $^{a}$ & No. & Rate $^{a}$ \\
\hline \multicolumn{11}{|c|}{ Female adult or adolescent } \\
\hline Injection drug use & 32,748 & - & 32,683 & - & 32,442 & - & 32,079 & - & 31,776 & - \\
\hline Heterosexual contact ${ }^{\mathrm{e}}$ & 81,913 & - & 84,741 & - & 87,349 & - & 89,102 & - & 90,849 & - \\
\hline Perinatal & 2,541 & - & 2,613 & - & 2,721 & - & 2,782 & - & 2,833 & - \\
\hline Other ${ }^{f}$ & 1,109 & - & 1,106 & - & 1,106 & - & 1,111 & - & 1,112 & - \\
\hline Subtotal & 118,311 & 88.1 & 121,143 & 89.4 & 123,618 & 90.5 & 125,074 & 90.8 & 126,569 & 91.1 \\
\hline \multicolumn{11}{|c|}{ Child ( $<13$ yrs at end of year) } \\
\hline Perinatal & 364 & - & 307 & - & 262 & - & 272 & - & 245 & - \\
\hline Other ${ }^{f}$ & 22 & - & 21 & - & 21 & - & 31 & - & 33 & - \\
\hline Subtotal & 386 & 0.7 & 328 & 0.6 & 283 & 0.5 & 303 & 0.6 & 278 & 0.5 \\
\hline \multicolumn{11}{|l|}{ Region of residence ${ }^{g}$} \\
\hline Northeast & 131,321 & 236.0 & 132,481 & 237.3 & 133,396 & 238.3 & 134,082 & 238.9 & 133,827 & 238.2 \\
\hline Midwest & 54,467 & 81.1 & 55,857 & 83.0 & 57,199 & 84.7 & 58,110 & 85.8 & 58,655 & 86.5 \\
\hline South & 204,202 & 175.9 & 211,541 & 180.3 & 218,324 & 184.4 & 222,083 & 185.5 & 226,408 & 187.1 \\
\hline West & 97,413 & 133.8 & 99,302 & 135.0 & 100,958 & 136.0 & 101,944 & 135.9 & 103,393 & 136.3 \\
\hline U.S. dependent areas & 9,550 & 234.5 & 9,553 & 237.2 & 9,648 & 242.1 & 9,599 & 244.4 & 9,559 & 247.2 \\
\hline Total $^{\mathrm{h}}$ & 496,953 & 157.4 & 508,734 & 160.0 & 519,525 & 162.3 & 525,818 & 163.0 & 531,842 & 163.8 \\
\hline
\end{tabular}


because of the lack of denominator data.

$\mathrm{b}$ Includes Asian/Pacific Islander legacy cases (see Technical Notes).

${ }^{\mathrm{C}}$ Hispanics/Latinos can be of any race.

${ }^{d}$ Data have been statistically adjusted to account for missing transmission category; therefore, values may not sum to column subtotals and total.

${ }^{\mathrm{e}}$ Heterosexual contact with a person known to have, or to be at high risk for, HIV infection.

${ }^{\mathrm{f}}$ Includes hemophilia, blood transfusion, and risk factor not reported or not identified.

9 Data are based on address of residence at the end of the specified year (i.e., most recent known address).

$\mathrm{h}_{\text {Includes persons whose race/ethnicity is unknown. }}$ 


\begin{tabular}{|c|c|c|c|c|c|c|c|c|c|c|c|c|c|c|c|c|}
\hline & \multicolumn{2}{|c|}{$\begin{array}{c}\text { American Indian/ } \\
\text { Alaska Native }\end{array}$} & \multicolumn{2}{|c|}{ Asian $^{a}$} & \multicolumn{2}{|c|}{$\begin{array}{c}\text { Black } / \\
\text { African American }\end{array}$} & \multicolumn{2}{|c|}{ Hispanic/Latino ${ }^{b}$} & \multicolumn{2}{|c|}{$\begin{array}{l}\text { Native Hawaiian/ } \\
\text { Other Pacific } \\
\text { Islander }\end{array}$} & \multicolumn{2}{|c|}{ White } & \multicolumn{2}{|c|}{ Multiple races } & \multicolumn{2}{|c|}{ Total $^{\mathrm{C}}$} \\
\hline & No. & Rate $^{d}$ & No. & Rate $^{d}$ & No. & Rate $^{d}$ & No. & Rate $^{d}$ & No. & Rate $^{d}$ & No. & Rate $^{d}$ & No. & Rate $^{d}$ & No. & Rate $^{d}$ \\
\hline \multicolumn{17}{|l|}{ Age at end of year (yr) } \\
\hline$<13$ & 8 & 1.8 & 100 & 3.9 & 1,482 & 20.5 & 308 & 2.3 & 3 & 2.8 & 297 & 1.1 & 124 & 5.3 & 2,322 & 4.4 \\
\hline $13-14$ & 5 & 7.1 & 12 & 3.0 & 464 & 40.5 & 107 & 5.5 & 1 & 6.3 & 88 & 2.0 & 44 & 15.0 & 721 & 8.7 \\
\hline 15-19 & 10 & 5.5 & 64 & 6.3 & 3,136 & 105.0 & 1,005 & 21.4 & 4 & 10.0 & 515 & 4.5 & 219 & 32.0 & 4,959 & 23.5 \\
\hline 20-24 & 117 & 58.8 & 397 & 31.8 & 17,951 & 529.1 & 6,619 & 138.3 & 33 & 71.4 & 4,369 & 35.2 & 1,318 & 219.7 & 30,812 & 135.8 \\
\hline $25-29$ & 221 & 120.6 & 973 & 66.6 & 34,802 & $1,118.9$ & 14,740 & 327.1 & 68 & 132.5 & 11,883 & 94.1 & 2,846 & 616.5 & 65,540 & 292.6 \\
\hline 30-34 & 284 & 174.5 & 1,299 & 87.4 & 35,345 & $1,278.9$ & 19,469 & 440.2 & 91 & 187.9 & 16,876 & 136.6 & 3,285 & 858.6 & 76,656 & 354.6 \\
\hline 35-39 & 314 & 212.0 & 1,627 & 116.7 & 37,647 & $1,446.6$ & 23,698 & 562.7 & 126 & 299.9 & 21,153 & 182.5 & 3,631 & $1,119.7$ & 88,220 & 434.3 \\
\hline $40-44$ & 340 & 237.3 & 2,147 & 154.0 & 42,577 & $1,671.6$ & 26,675 & 683.9 & 118 & 318.7 & 27,570 & 232.5 & 4,100 & $1,470.8$ & 103,583 & 513.9 \\
\hline $45-49$ & 483 & 328.5 & 2,099 & 170.3 & 56,397 & $2,184.0$ & 34,333 & 990.6 & 131 & 374.8 & 46,946 & 358.6 & 5,827 & 2,347.6 & 146,349 & 703.6 \\
\hline $50-54$ & 469 & 289.5 & 1,620 & 144.1 & 64,045 & $2,364.2$ & 34,982 & 1,158.4 & 127 & 366.8 & 63,042 & 420.3 & 6,669 & $2,766.4$ & 171,172 & 767.9 \\
\hline $55-59$ & 320 & 209.2 & 1,110 & 108.5 & 52,280 & $2,047.1$ & 24,592 & $1,011.4$ & 91 & 299.1 & 47,988 & 312.5 & 4,847 & 2,217.3 & 131,430 & 603.8 \\
\hline $60-64$ & 199 & 160.8 & 709 & 80.7 & 32,877 & $1,597.4$ & 14,351 & 786.0 & 59 & 249.3 & 30,248 & 216.7 & 2,852 & 1,663.6 & 81,438 & 427.8 \\
\hline$\geq 65$ & 134 & 54.6 & 730 & 36.7 & 26,854 & 638.8 & 12,857 & 342.7 & 39 & 85.0 & 27,695 & 74.5 & 2,172 & 632.3 & 70,644 & 148.0 \\
\hline \multicolumn{17}{|l|}{ Transmission category } \\
\hline \multicolumn{17}{|l|}{ Male adult or adolescent } \\
\hline Male-to-male sexual contact & 1,458 & - & 8,706 & - & 162,191 & - & 120,755 & - & 632 & - & 212,143 & - & 20,096 & - & 526,456 & - \\
\hline Injection drug use & 218 & - & 429 & - & 37,008 & - & 21,226 & - & 26 & - & 13,973 & - & 2,591 & - & 75,569 & - \\
\hline $\begin{array}{l}\text { Male-to-male sexual contact and } \\
\text { injection drug use }\end{array}$ & 276 & - & 364 & - & 15,835 & - & 11,393 & - & 38 & - & 21,815 & - & 3,310 & - & 53,090 & - \\
\hline Heterosexual contact ${ }^{f}$ & 164 & - & 904 & - & 47,832 & - & 15,307 & - & 36 & - & 9,429 & - & 2,327 & - & 76,038 & - \\
\hline Perinatal & 7 & - & 25 & - & 2,631 & - & 1,174 & - & 2 & - & 548 & - & 224 & - & 4,621 & - \\
\hline Other ${ }^{9}$ & 13 & - & 76 & - & 880 & - & 487 & - & 2 & - & 1,474 & - & 119 & - & 3,058 & - \\
\hline Subtotal & 2,136 & 227.3 & 10,504 & 152.6 & 266,377 & $1,730.3$ & 170,342 & 786.9 & 735 & 324.5 & 259,381 & 309.2 & 28,667 & 1,398.0 & 738,832 & 563.9 \\
\hline
\end{tabular}




\begin{tabular}{|c|c|c|c|c|c|c|c|c|c|c|c|c|c|c|c|c|}
\hline & \multicolumn{2}{|c|}{$\begin{array}{l}\text { American Indian/ } \\
\text { Alaska Native }\end{array}$} & \multicolumn{2}{|c|}{ Asian $^{a}$} & \multicolumn{2}{|c|}{$\begin{array}{c}\text { Black/ } \\
\text { African American }\end{array}$} & \multicolumn{2}{|c|}{ Hispanic/Latino ${ }^{b}$} & \multicolumn{2}{|c|}{$\begin{array}{l}\text { Native Hawaiian/ } \\
\text { Other Pacific } \\
\text { Islander }\end{array}$} & \multicolumn{2}{|c|}{ White } & \multicolumn{2}{|c|}{ Multiple races } & \multicolumn{2}{|c|}{ Total $^{c}$} \\
\hline & No. & Rate $^{d}$ & No. & Rate $^{d}$ & No. & Rate $^{d}$ & No. & Rate $^{d}$ & No. & Rate $^{d}$ & No. & Rate $^{d}$ & No. & Rate $^{d}$ & No. & Rate $^{d}$ \\
\hline \multicolumn{17}{|c|}{ Female adult or adolescent } \\
\hline Injection drug use & 234 & - & 175 & - & 25,812 & - & 9,827 & - & 26 & - & 12,368 & - & 2,599 & - & 51,135 & - \\
\hline Heterosexual contact ${ }^{f}$ & 505 & - & 2,022 & - & 108,264 & - & 31,730 & - & 125 & - & 25,542 & - & 6,224 & - & 174,577 & - \\
\hline Perinatal & 8 & - & 22 & - & 3,048 & - & 1,206 & - & 1 & - & 577 & - & 238 & - & 5,107 & - \\
\hline Otherg & 13 & - & 64 & - & 874 & - & 323 & - & 1 & - & 505 & - & 82 & - & 1,873 & - \\
\hline Subtotal & 760 & 77.5 & 2,283 & 29.4 & 137,998 & 799.5 & 43,086 & 202.2 & 153 & 68.3 & 38,992 & 44.6 & 9,143 & 416.0 & 232,692 & 169.7 \\
\hline \multicolumn{17}{|c|}{ Child (<13 yrs at end of year) } \\
\hline Perinatal & 8 & - & 69 & - & 1,206 & - & 279 & - & 3 & - & 198 & - & 109 & - & 1,872 & - \\
\hline Other $^{g}$ & 0 & - & 31 & - & 276 & - & 29 & - & 0 & - & 99 & - & 15 & - & 450 & - \\
\hline Subtotal & 8 & 1.8 & 100 & 3.9 & 1,482 & 20.5 & 308 & 2.3 & 3 & 2.8 & 297 & 1.1 & 124 & 5.3 & 2,322 & 4.4 \\
\hline \multicolumn{17}{|l|}{ Region of residence ${ }^{h}$} \\
\hline Northeast & 149 & 118.2 & 2,692 & 74.3 & 89,132 & 1408.2 & 76,531 & 971.8 & 58 & 277.0 & 53,390 & 143.1 & 12,250 & $1,351.6$ & 234,735 & 417.8 \\
\hline Midwest & 354 & 87.2 & 1,261 & 60.0 & 50,056 & 711.3 & 12,985 & 251.3 & 38 & 122.1 & 46,261 & 89.2 & 4,660 & 371.5 & 115,744 & 170.6 \\
\hline South & 721 & 91.9 & 2,565 & 64.3 & 236,828 & $1,026.6$ & 65,950 & 316.4 & 148 & 174.8 & 113,214 & 161.5 & 15,246 & 707.0 & 434,853 & 359.3 \\
\hline West & 1,680 & 159.5 & 6,369 & 84.8 & 29,841 & 867.5 & 58,270 & 259.5 & 647 & 154.3 & 85,805 & 221.7 & 5,778 & 256.4 & 188,514 & 248.6 \\
\hline Total & 2,904 & 122.6 & 12,887 & 74.8 & 405,857 & $1,017.8$ & 213,736 & 379.4 & 891 & 160.3 & 298,670 & 150.9 & 37,934 & 577.3 & 973,846 & 303.5 \\
\hline \multicolumn{17}{|c|}{ Note. Numbers less than 12, and rates based on these numbers, should be interpreted with caution. } \\
\hline \multicolumn{17}{|c|}{ a Includes Asian/Pacific Islander legacy cases (see Technical Notes). } \\
\hline \multicolumn{17}{|c|}{ b Hispanics/Latinos can be of any race. } \\
\hline \multicolumn{17}{|c|}{${ }^{\mathrm{C}}$ Includes persons whose race/ethnicity is unknown. } \\
\hline \multicolumn{17}{|c|}{ d Rates are per 100,000 population. Rates are not calculated by transmission category because of the lack of denominator data. } \\
\hline \multicolumn{17}{|c|}{ e Data have been statistically adjusted to account for missing transmission category; therefore, values may not sum to column subtotals and total. } \\
\hline \multicolumn{17}{|c|}{ f Heterosexual contact with a person known to have, or to be at high risk for, HIV infection. } \\
\hline \multicolumn{17}{|c|}{$\mathrm{g}$ Includes hemophilia, blood transfusion, and risk factor not reported or not identified. } \\
\hline${ }^{\mathrm{h}}$ Data are based on addre & the end 0 & he specifie & year (i.e., $m$ & t recent $k$ & wn address & & & & & & & & & & & \\
\hline
\end{tabular}




\begin{tabular}{|c|c|c|c|c|c|c|c|c|}
\hline & $\begin{array}{c}\text { American Indian/ } \\
\text { Alaska Native } \\
\text { No. }\end{array}$ & $\begin{array}{c}\text { Asian }^{\mathrm{a}} \\
\text { No. }\end{array}$ & $\begin{array}{c}\text { Black/ } \\
\text { African American } \\
\text { No. }\end{array}$ & $\begin{array}{c}\text { Hispanic/Latino } \\
\text { No. }\end{array}$ & $\begin{array}{c}\text { Native Hawaiian/ } \\
\text { Other Pacific } \\
\text { Islander } \\
\text { No. }\end{array}$ & $\begin{array}{c}\text { White } \\
\text { No. }\end{array}$ & $\begin{array}{c}\text { Multiple races } \\
\text { No. }\end{array}$ & $\begin{array}{c}\text { Total }^{c} \\
\text { No. }\end{array}$ \\
\hline \multicolumn{9}{|l|}{ Age at end of year (yr) } \\
\hline$<13$ & 8 & 100 & 1,483 & 317 & 4 & 297 & 125 & 2,334 \\
\hline 13-14 & 5 & 12 & 464 & 113 & 1 & 88 & 44 & 727 \\
\hline $15-19$ & 10 & 64 & 3,139 & 1,069 & 4 & 515 & 219 & 5,026 \\
\hline $20-24$ & 117 & 397 & 17,956 & 7,008 & 33 & 4,369 & 1,320 & 31,208 \\
\hline $25-29$ & 221 & 977 & 34,816 & 15,498 & 73 & 11,885 & 2,847 & 66,324 \\
\hline $30-34$ & 284 & 1,301 & 35,361 & 20,414 & 95 & 16,880 & 3,285 & 77,627 \\
\hline $35-39$ & 314 & 1,632 & 37,667 & 25,170 & 130 & 21,158 & 3,634 & 89,729 \\
\hline 40-44 & 340 & 2,154 & 42,619 & 28,461 & 122 & 27,574 & 4,100 & 105,428 \\
\hline $45-49$ & 483 & 2,108 & 56,453 & 36,974 & 134 & 46,960 & 5,829 & 149,074 \\
\hline $50-54$ & 469 & 1,626 & 64,085 & 38,120 & 127 & 63,056 & 6,671 & 174,372 \\
\hline $55-59$ & 321 & 1,114 & 52,324 & 27,083 & 93 & 48,000 & 4,847 & 133,984 \\
\hline $60-64$ & 199 & 712 & 32,906 & 15,938 & 61 & 30,256 & 2,852 & 83,068 \\
\hline$\geq 65$ & 134 & 735 & 26,902 & 14,536 & 40 & 27,706 & 2,172 & 72,388 \\
\hline \multicolumn{9}{|l|}{ Transmission category $^{d}$} \\
\hline \multicolumn{9}{|l|}{ Male adult or adolescent } \\
\hline Male-to-male sexual contact & 1,458 & 8,732 & 162,264 & 125,101 & 646 & 212,190 & 20,102 & 530,970 \\
\hline Injection drug use & 218 & 436 & 37,047 & 25,202 & 27 & 13,978 & 2,591 & 79,599 \\
\hline $\begin{array}{l}\text { Male-to-male sexual contact } \\
\text { and injection drug use }\end{array}$ & 276 & 364 & 15,842 & 12,188 & 38 & 21,819 & 3,311 & 53,898 \\
\hline Heterosexual contact ${ }^{\mathrm{e}}$ & 165 & 906 & 47,894 & 17,812 & 38 & 9,430 & 2,327 & 78,612 \\
\hline Perinatal & 7 & 25 & 2,634 & 1,294 & 2 & 548 & 224 & 4,744 \\
\hline Other ${ }^{f}$ & 13 & 76 & 880 & 527 & 2 & 1,474 & 119 & 3,098 \\
\hline Subtotal & 2,137 & 10,540 & 266,561 & 182,124 & 753 & 259,440 & 28,674 & 750,921 \\
\hline
\end{tabular}




\begin{tabular}{|c|c|c|c|c|c|c|c|c|}
\hline & $\begin{array}{c}\text { American Indian/ } \\
\text { Alaska Native } \\
\text { No. }\end{array}$ & $\begin{array}{l}\text { Asian }{ }^{a} \\
\text { No. }\end{array}$ & $\begin{array}{c}\text { Black/ } \\
\text { African American } \\
\text { No. }\end{array}$ & $\begin{array}{c}\text { Hispanic/Latino }^{b} \\
\text { No. }\end{array}$ & $\begin{array}{c}\text { Native Hawaiian/ } \\
\text { Other Pacific } \\
\text { Islander } \\
\text { No. } \\
\end{array}$ & $\begin{array}{c}\text { White } \\
\text { No. }\end{array}$ & $\begin{array}{c}\text { Multiple races } \\
\text { No. }\end{array}$ & $\begin{array}{l}\text { Total }^{\mathrm{C}} \\
\text { No. }\end{array}$ \\
\hline \multicolumn{9}{|c|}{ Female adult or adolescent } \\
\hline Injection drug use & 234 & 177 & 25,827 & 10,956 & 26 & 12,373 & 2,599 & 52,288 \\
\hline Heterosexual contact ${ }^{e}$ & 505 & 2,029 & 108,373 & 35,644 & 131 & 25,552 & 6,227 & 178,626 \\
\hline Perinatal & 8 & 22 & 3,053 & 1,313 & 1 & 577 & 238 & 5,219 \\
\hline Other ${ }^{f}$ & 13 & 64 & 878 & 347 & 1 & 505 & 82 & 1,901 \\
\hline Subtotal & 760 & 2,292 & 138,131 & 48,260 & 160 & 39,007 & 9,146 & 238,034 \\
\hline \multicolumn{9}{|c|}{ Child (<13 yrs at end of year) } \\
\hline Perinatal & 8 & 69 & 1,207 & 288 & 4 & 198 & 110 & 1,884 \\
\hline Other $^{f}$ & 0 & 31 & 276 & 29 & 0 & 99 & 15 & 450 \\
\hline Subtotal & 8 & 100 & 1,483 & 317 & 4 & 297 & 125 & 2,334 \\
\hline \multicolumn{9}{|l|}{ Region of residence ${ }^{g}$} \\
\hline Northeast & 149 & 2,692 & 89,132 & 76,531 & 58 & 53,390 & 12,250 & 234,735 \\
\hline Midwest & 354 & 1,261 & 50,056 & 12,985 & 38 & 46,261 & 4,660 & 115,744 \\
\hline South & 721 & 2,565 & 236,828 & 65,950 & 148 & 113,214 & 15,246 & 434,853 \\
\hline West & 1,680 & 6,369 & 29,841 & 58,270 & 647 & 85,805 & 5,778 & 188,514 \\
\hline U.S. dependent areas & 1 & 45 & 318 & 16,965 & 26 & 74 & 11 & 17,443 \\
\hline Total & 2,905 & 12,932 & 406,175 & 230,701 & 917 & 298,744 & 37,945 & 991,289 \\
\hline \multicolumn{9}{|c|}{ Note. Numbers less than 12 should be interpreted with caution. } \\
\hline \multicolumn{9}{|c|}{ a Includes Asian/Pacific Islander legacy cases (see Technical Notes). } \\
\hline \multicolumn{9}{|c|}{${ }^{b}$ Hispanics/Latinos can be of any race. } \\
\hline \multicolumn{9}{|c|}{${ }^{\mathrm{C}}$ Includes persons whose race/ethnicity is unknown. } \\
\hline \multicolumn{9}{|c|}{ d Data have been statistically adjusted to account for missing transmission category; therefore, values may not sum to column subtotals and total. } \\
\hline \multicolumn{9}{|c|}{${ }^{\mathrm{e}}$ Heterosexual contact with a person known to have, or to be at high risk for, HIV infection. } \\
\hline \multicolumn{9}{|c|}{ f Includes hemophilia, blood transfusion, and risk factor not reported or not identified. } \\
\hline${ }^{9}$ Data are based on addres & idence at the end of the & year (i.e., $n$ & nt known address). & & & & & \\
\hline
\end{tabular}




\begin{tabular}{|c|c|c|c|c|c|c|c|c|c|c|c|c|c|c|c|c|}
\hline & \multicolumn{2}{|c|}{$\begin{array}{c}\text { American Indian/ } \\
\text { Alaska Native }\end{array}$} & \multicolumn{2}{|c|}{ Asian $^{\mathrm{a}}$} & \multicolumn{2}{|c|}{$\begin{array}{l}\text { Black/ } \\
\text { African American }\end{array}$} & \multicolumn{2}{|c|}{ Hispanic/Latino ${ }^{b}$} & \multicolumn{2}{|c|}{$\begin{array}{l}\text { Native Hawaiian/ } \\
\text { Other Pacific } \\
\text { Islander }\end{array}$} & \multicolumn{2}{|c|}{ White } & \multicolumn{2}{|c|}{ Multiple races } & \multicolumn{2}{|c|}{ Total $^{\mathrm{C}}$} \\
\hline & No. & Rate $^{d}$ & No. & Rate $^{d}$ & No. & Rate $^{d}$ & No. & Rate $^{d}$ & No. & Rate $^{\mathrm{d}}$ & No. & Rate $^{d}$ & No. & Rate $^{d}$ & No. & Rate $^{d}$ \\
\hline \multicolumn{17}{|l|}{ Age at end of year (yr) } \\
\hline$<13$ & 0 & 0.0 & 5 & 0.2 & 179 & 2.5 & 45 & 0.3 & 1 & 0.9 & 27 & 0.1 & 19 & 0.8 & 276 & 0.5 \\
\hline $13-14$ & 3 & 4.2 & 4 & 1.0 & 83 & 7.2 & 20 & 1.0 & 1 & 6.3 & 16 & 0.4 & 5 & 1.7 & 132 & 1.6 \\
\hline 15-19 & 2 & 1.1 & 9 & 0.9 & 651 & 21.8 & 185 & 3.9 & 0 & 0.0 & 79 & 0.7 & 46 & 6.7 & 972 & 4.6 \\
\hline 20-24 & 23 & 11.6 & 52 & 4.2 & 4,209 & 124.1 & 1,405 & 29.4 & 8 & 17.3 & 652 & 5.2 & 386 & 64.3 & 6,736 & 29.7 \\
\hline $25-29$ & 54 & 29.5 & 195 & 13.3 & 10,358 & 333.0 & 4,111 & 91.2 & 17 & 33.1 & 2,379 & 18.8 & 903 & 195.6 & 18,017 & 80.4 \\
\hline 30-34 & 108 & 66.4 & 391 & 26.3 & 13,410 & 485.2 & 7,407 & 167.5 & 30 & 62.0 & 4,618 & 37.4 & 1,294 & 338.2 & 27,258 & 126.1 \\
\hline 35-39 & 129 & 87.1 & 628 & 45.0 & 17,254 & 663.0 & 11,057 & 262.5 & 58 & 138.0 & 7,669 & 66.2 & 1,811 & 558.5 & 38,606 & 190.1 \\
\hline 40-44 & 183 & 127.7 & 1,011 & 72.5 & 22,820 & 895.9 & 14,741 & 377.9 & 55 & 148.5 & 12,480 & 105.3 & 2,421 & 868.5 & 53,713 & 266.5 \\
\hline $45-49$ & 277 & 188.4 & 1,163 & 94.4 & 33,370 & $1,292.2$ & 21,226 & 612.5 & 86 & 246.0 & 24,848 & 189.8 & 3,768 & $1,518.1$ & 84,744 & 407.4 \\
\hline $50-54$ & 280 & 172.9 & 1,004 & 89.3 & 40,004 & $1,476.7$ & 23,647 & 783.1 & 85 & 245.5 & 37,071 & 247.2 & 4,665 & $1,935.1$ & 106,763 & 479.0 \\
\hline 55-59 & 212 & 138.6 & 737 & 72.0 & 33,605 & $1,315.9$ & 17,202 & 707.5 & 49 & 161.1 & 29,459 & 191.8 & 3,427 & $1,567.7$ & 84,701 & 389.1 \\
\hline $60-64$ & 130 & 105.0 & 495 & 56.3 & 21,502 & $1,044.7$ & 10,372 & 568.1 & 33 & 139.4 & 18,962 & 135.9 & 2,038 & $1,188.8$ & 53,541 & 281.2 \\
\hline$\geq 65$ & 91 & 37.1 & 517 & 26.0 & 17,579 & 418.1 & 9,352 & 249.3 & 29 & 63.2 & 17,698 & 47.6 & 1,548 & 450.6 & 46,824 & 98.1 \\
\hline \multicolumn{17}{|l|}{ Transmission category } \\
\hline \multicolumn{17}{|l|}{ Male adult or adolescent } \\
\hline Male-to-male sexual contact & 693 & - & 4,030 & - & 77,173 & - & 62,513 & - & 323 & - & 108,207 & - & 10,825 & - & 263,791 & - \\
\hline Injection drug use & 132 & - & 253 & - & 24,267 & - & 14,787 & - & 11 & - & 8,759 & - & 1,927 & - & 50,139 & - \\
\hline $\begin{array}{l}\text { Male-to-male sexual contact and } \\
\text { injection drug use }\end{array}$ & 174 & - & 224 & - & 10,435 & - & 7,524 & - & 23 & - & 12,972 & - & 2,264 & - & 33,617 & - \\
\hline Heterosexual contact $f^{f}$ & 96 & - & 561 & - & 28,239 & - & 10,242 & - & 19 & - & 5,472 & - & 1,552 & - & 46,187 & - \\
\hline Perinatal & 3 & - & 11 & - & 1,445 & - & 652 & - & 2 & - & 274 & - & 126 & - & 2,513 & - \\
\hline Other ${ }^{g}$ & 12 & - & 56 & - & 487 & - & 343 & - & 1 & - & 1,072 & - & 82 & - & 2,053 & - \\
\hline Subtotal & 1,110 & 118.1 & 5,135 & 74.6 & 142,045 & 922.7 & 96,061 & 443.8 & 379 & 167.3 & 136,756 & 163.0 & 16,776 & 818.1 & 398,300 & 304.0 \\
\hline
\end{tabular}




\begin{tabular}{|c|c|c|c|c|c|c|c|c|c|c|c|c|c|c|c|c|}
\hline & \multicolumn{2}{|c|}{$\begin{array}{l}\text { American Indian/ } \\
\text { Alaska Native }\end{array}$} & \multicolumn{2}{|c|}{ Asian $^{a}$} & \multicolumn{2}{|c|}{$\begin{array}{c}\text { Black/ } \\
\text { African American }\end{array}$} & \multicolumn{2}{|c|}{ Hispanic/Latino ${ }^{b}$} & \multicolumn{2}{|c|}{$\begin{array}{l}\text { Native Hawaiian/ } \\
\text { Other Pacific } \\
\text { Islander }\end{array}$} & \multicolumn{2}{|c|}{ White } & \multicolumn{2}{|c|}{ Multiple races } & \multicolumn{2}{|c|}{ Total $^{\mathrm{C}}$} \\
\hline & No. & Rate $^{d}$ & No. & Rate $^{d}$ & No. & Rate $^{d}$ & No. & Rate $^{d}$ & No. & Rate $^{d}$ & No. & Rate $^{d}$ & No. & Rate $^{d}$ & No. & Rate $^{d}$ \\
\hline \multicolumn{17}{|c|}{ Female adult or adolescent } \\
\hline Injection drug use & 117 & - & 91 & - & 15,960 & - & 6,448 & - & 16 & - & 6,635 & - & 1,805 & - & 31,072 & - \\
\hline Heterosexual contact $^{f}$ & 252 & - & 929 & - & 54,701 & - & 17,331 & - & 54 & - & 11,951 & - & 3,555 & - & 88,777 & - \\
\hline Perinatal & 5 & - & 9 & - & 1,661 & - & 675 & - & 1 & - & 274 & - & 133 & - & 2,759 & - \\
\hline Other ${ }^{g}$ & 9 & - & 42 & - & 478 & - & 211 & - & 1 & - & 315 & - & 43 & - & 1,099 & - \\
\hline Subtotal & 382 & 38.9 & 1,071 & 13.8 & 72,800 & 421.8 & 24,664 & 115.7 & 72 & 32.1 & 19,175 & 21.9 & 5,536 & 251.9 & 123,707 & 90.2 \\
\hline \multicolumn{17}{|c|}{ Child ( $<13$ yrs at end of year) } \\
\hline Perinatal & 0 & - & 4 & - & 160 & - & 39 & - & 1 & - & 22 & - & 17 & - & 243 & - \\
\hline Otherg & 0 & - & 1 & - & 19 & - & 6 & - & 0 & - & 5 & - & 2 & - & 33 & - \\
\hline Subtotal & 0 & 0.0 & 5 & 0.2 & 179 & 2.5 & 45 & 0.3 & 1 & 0.9 & 27 & 0.1 & 19 & 0.8 & 276 & 0.5 \\
\hline \multicolumn{17}{|l|}{ Region of residence $^{h}$} \\
\hline Northeast & 77 & 61.1 & 1,303 & 35.9 & 50,697 & 801.0 & 45,701 & 580.3 & 21 & 100.3 & 28,350 & 76.0 & 7,652 & 844.3 & 133,827 & 238.2 \\
\hline Midwest & 170 & 41.9 & 552 & 26.3 & 25,015 & 355.4 & 7,080 & 137.0 & 17 & 54.6 & 23,169 & 44.7 & 2,651 & 211.3 & 58,655 & 86.5 \\
\hline South & 330 & 42.1 & 1,084 & 27.2 & 123,219 & 534.1 & 34,973 & 167.8 & 49 & 57.9 & 57,941 & 82.6 & 8,807 & 408.4 & 226,408 & 187.1 \\
\hline West & 915 & 86.9 & 3,272 & 43.6 & 16,093 & 467.8 & 33,016 & 147.0 & 365 & 87.1 & 46,498 & 120.1 & 3,221 & 142.9 & 103,393 & 136.3 \\
\hline Total & 1,492 & 63.0 & 6,211 & 36.1 & 215,024 & 539.2 & 120,770 & 214.4 & 452 & 81.3 & 155,958 & 78.8 & 22,331 & 339.9 & 522,283 & 162.8 \\
\hline \multicolumn{17}{|c|}{ Note. Numbers less than 12 , and rates based on these numbers, should be interpreted with caution. } \\
\hline \multicolumn{17}{|c|}{ a Includes Asian/Pacific Islander legacy cases (see Technical Notes). } \\
\hline \multicolumn{17}{|c|}{ b Hispanics/Latinos can be of any race. } \\
\hline \multicolumn{17}{|c|}{${ }^{\mathrm{C}}$ Includes persons whose race/ethnicity is unknown. } \\
\hline \multicolumn{17}{|c|}{${ }^{d}$ Rates are per 100,000 population. Rates are not calculated by transmission category because of the lack of denominator data. } \\
\hline \multicolumn{17}{|c|}{ e Data have been statistically adjusted to account for missing transmission category; therefore, values may not sum to column subtotals and total. } \\
\hline \multicolumn{17}{|c|}{${ }^{f}$ Heterosexual contact with a person known to have, or to be at high risk for, HIV infection. } \\
\hline \multicolumn{17}{|c|}{9 Includes hemophilia, blood transfusion, and risk factor not reported or not identified. } \\
\hline${ }^{\mathrm{h}}$ Data are based on addres & $t$ the end 0 & ne specifie & ear (i.e., $n$ & st recent 1 & wn address & & & & & & & & & & & \\
\hline
\end{tabular}




\begin{tabular}{|c|c|c|c|c|c|c|c|c|}
\hline & $\begin{array}{c}\text { American Indian/ } \\
\text { Alaska Native } \\
\text { No. }\end{array}$ & $\begin{array}{l}\text { Asian }^{\mathrm{a}} \\
\text { No. }\end{array}$ & $\begin{array}{c}\text { Black/ } \\
\text { African American } \\
\text { No. }\end{array}$ & $\begin{array}{c}\text { Hispanic/Latino } \\
\text { No. }\end{array}$ & $\begin{array}{c}\text { Native Hawaiian/ } \\
\text { Other Pacific } \\
\text { Islander } \\
\text { No. }\end{array}$ & $\begin{array}{c}\text { White } \\
\text { No. }\end{array}$ & $\begin{array}{c}\text { Multiple races } \\
\text { No. }\end{array}$ & $\begin{array}{c}\text { Total }^{\mathrm{C}} \\
\text { No. }\end{array}$ \\
\hline \multicolumn{9}{|l|}{ Age at end of year } \\
\hline$<13$ & 0 & 5 & 179 & 46 & 2 & 27 & 19 & 278 \\
\hline 13-14 & 3 & 4 & 83 & 21 & 1 & 16 & 5 & 133 \\
\hline $15-19$ & 2 & 9 & 653 & 202 & 0 & 79 & 46 & 991 \\
\hline $20-24$ & 23 & 52 & 4,212 & 1,493 & 8 & 652 & 386 & 6,827 \\
\hline $25-29$ & 54 & 196 & 10,361 & 4,304 & 19 & 2,379 & 903 & 18,216 \\
\hline $30-34$ & 108 & 391 & 13,417 & 7,677 & 30 & 4,619 & 1,294 & 27,536 \\
\hline $35-39$ & 129 & 629 & 17,262 & 11,580 & 61 & 7,672 & 1,811 & 39,144 \\
\hline $40-44$ & 183 & 1,013 & 22,840 & 15,622 & 57 & 12,480 & 2,421 & 54,618 \\
\hline $45-49$ & 277 & 1,168 & 33,406 & 22,692 & 88 & 24,852 & 3,769 & 86,258 \\
\hline $50-54$ & 280 & 1,007 & 40,027 & 25,623 & 85 & 37,082 & 4,667 & 108,778 \\
\hline $55-59$ & 213 & 738 & 33,625 & 18,874 & 50 & 29,466 & 3,427 & 86,403 \\
\hline $60-64$ & 130 & 497 & 21,524 & 11,448 & 35 & 18,966 & 2,038 & 54,647 \\
\hline$\geq 65$ & 91 & 519 & 17,606 & 10,503 & 30 & 17,706 & 1,548 & 48,013 \\
\hline \multicolumn{9}{|l|}{ Transmission category ${ }^{d}$} \\
\hline \multicolumn{9}{|l|}{ Male adult or adolescent } \\
\hline Male-to-male sexual contact & 693 & 4,042 & 77,217 & 64,607 & 330 & 108,234 & 10,827 & 265,977 \\
\hline Injection drug use & 132 & 255 & 24,284 & 17,188 & 12 & 8,761 & 1,927 & 52,563 \\
\hline $\begin{array}{l}\text { Male-to-male sexual contact } \\
\text { and injection drug use }\end{array}$ & 174 & 224 & 10,439 & 8,041 & 23 & 12,974 & 2,264 & 34,141 \\
\hline Heterosexual contact ${ }^{\mathrm{e}}$ & 97 & 561 & 28,280 & 11,664 & 20 & 5,473 & 1,552 & 47,653 \\
\hline Perinatal & 3 & 11 & 1,447 & 726 & 2 & 274 & 126 & 2,589 \\
\hline Other ${ }^{f}$ & 12 & 56 & 487 & 363 & 1 & 1,072 & 82 & 2,073 \\
\hline Subtotal & 1,111 & 5,149 & 142,154 & 102,589 & 388 & 136,788 & 16,778 & 404,995 \\
\hline
\end{tabular}


Table 23b. Persons living with diagnosed HIV infection ever classified as stage 3 (AIDS), by race/ethnicity and selected characteristics, year-end 2015-United States and 6 dependent areas (cont)

\begin{tabular}{|c|c|c|c|c|c|c|c|c|}
\hline & $\begin{array}{c}\text { American Indian/ } \\
\text { Alaska Native } \\
\text { No. }\end{array}$ & $\begin{array}{c}\text { Asian }^{\mathrm{a}} \\
\text { No. }\end{array}$ & $\begin{array}{c}\text { Black/ } \\
\text { African American } \\
\text { No. }\end{array}$ & $\begin{array}{c}\text { Hispanic/Latino } \\
\text { No. }\end{array}$ & $\begin{array}{c}\text { Native Hawaiian/ } \\
\text { Other Pacific } \\
\text { Islander } \\
\text { No. }\end{array}$ & $\begin{array}{l}\text { White } \\
\text { No. }\end{array}$ & $\begin{array}{c}\text { Multiple races } \\
\text { No. }\end{array}$ & $\begin{array}{l}\text { Total }^{\mathrm{C}} \\
\text { No. }\end{array}$ \\
\hline \multicolumn{9}{|c|}{ Female adult or adolescent } \\
\hline Injection drug use & 117 & 91 & 15,968 & 7,139 & 17 & 6,638 & 1,805 & 31,776 \\
\hline Heterosexual contact & 252 & 932 & 54,750 & 19,344 & 58 & 11,954 & 3,556 & 90,849 \\
\hline Perinatal & 5 & 9 & 1,665 & 745 & 1 & 274 & 133 & 2,833 \\
\hline Other ${ }^{f}$ & 9 & 42 & 479 & 222 & 1 & 315 & 43 & 1,112 \\
\hline Subtotal & 382 & 1,074 & 72,862 & 27,450 & 76 & 19,181 & 5,537 & 126,569 \\
\hline \multicolumn{9}{|l|}{$\begin{array}{l}\text { Child }(<13 \text { yrs at end of } \\
\text { year) }\end{array}$} \\
\hline Perinatal & 0 & 4 & 160 & 40 & 2 & 22 & 17 & 245 \\
\hline Other ${ }^{f}$ & 0 & 1 & 19 & 6 & 0 & 5 & 2 & 33 \\
\hline Subtotal & 0 & 5 & 179 & 46 & 2 & 27 & 19 & 278 \\
\hline \multicolumn{9}{|l|}{ Region of residence $^{g}$} \\
\hline Northeast & 77 & 1,303 & 50,697 & 45,701 & 21 & 28,350 & 7,652 & 133,827 \\
\hline Midwest & 170 & 552 & 25,015 & 7,080 & 17 & 23,169 & 2,651 & 58,655 \\
\hline South & 330 & 1,084 & 123,219 & 34,973 & 49 & 57,941 & 8,807 & 226,408 \\
\hline West & 915 & 3,272 & 16,093 & 33,016 & 365 & 46,498 & 3,221 & 103,393 \\
\hline U.S. dependent areas & 1 & 17 & 171 & 9,315 & 14 & 38 & 3 & 9,559 \\
\hline Total & 1,493 & 6,228 & 215,195 & 130,085 & 466 & 155,996 & 22,334 & 531,842 \\
\hline
\end{tabular}

Note. Numbers less than 12 should be interpreted with caution.

a Includes Asian/Pacific Islander legacy cases (see Technical Notes).

${ }^{\mathrm{b}}$ Hispanics/Latinos can be of any race.

${ }^{\mathrm{C}}$ Includes persons whose race/ethnicity is unknown.

${ }^{d}$ Data have been statistically adjusted to account for missing transmission category; therefore, values may not sum to column subtotals and total.

${ }^{e}$ Heterosexual contact with a person known to have, or to be at high risk for, HIV infection.

f Includes hemophilia, blood transfusion, and risk factor not reported or not identified.

9 Data are based on address of residence at the end of the specified year (i.e., most recent known address). 
Table 24. Diagnoses of HIV infection, by area of residence, 2015 and 2016-United States and 6 dependent areas

\begin{tabular}{|c|c|c|c|c|c|c|c|c|c|c|c|c|}
\hline \multirow[b]{3}{*}{ Area of residence } & \multicolumn{6}{|c|}{2015} & \multicolumn{6}{|c|}{2016} \\
\hline & \multicolumn{2}{|c|}{$\begin{array}{l}\text { Adults and } \\
\text { adolescents }\end{array}$} & \multicolumn{2}{|c|}{ Children } & \multicolumn{2}{|c|}{ Total } & \multicolumn{2}{|c|}{$\begin{array}{l}\text { Adults and } \\
\text { adolescents }\end{array}$} & \multicolumn{2}{|c|}{ Children } & \multicolumn{2}{|c|}{ Total } \\
\hline & No. & Rate $^{a}$ & No. & Rate $^{a}$ & No. & Rate $^{a}$ & No. & Rate $^{a}$ & No. & Rate $^{a}$ & No. & Rate $^{a}$ \\
\hline Alabama & 658 & 16.2 & 0 & 0.0 & 658 & 13.6 & 533 & 13.1 & 0 & 0.0 & 533 & 11.0 \\
\hline Alaska & 24 & 4.0 & 0 & 0.0 & 24 & 3.3 & 37 & 6.1 & 0 & 0.0 & 37 & 5.0 \\
\hline Arizona & 695 & 12.3 & 4 & 0.3 & 699 & 10.3 & 778 & 13.5 & 1 & 0.1 & 779 & 11.2 \\
\hline Arkansas & 271 & 11.0 & 0 & 0.0 & 271 & 9.1 & 314 & 12.7 & 1 & 0.2 & 315 & 10.5 \\
\hline California & 4,983 & 15.4 & 8 & 0.1 & 4,991 & 12.8 & 4,961 & 15.2 & 11 & 0.2 & 4,972 & 12.7 \\
\hline Colorado & 378 & 8.3 & 1 & 0.1 & 379 & 7.0 & 423 & 9.1 & 0 & 0.0 & 423 & 7.6 \\
\hline Connecticut & 273 & 8.9 & 2 & 0.4 & 275 & 7.7 & 251 & 8.2 & 2 & 0.4 & 253 & 7.1 \\
\hline Delaware & 104 & 13.0 & 0 & 0.0 & 104 & 11.0 & 117 & 14.5 & 0 & 0.0 & 117 & 12.3 \\
\hline District of Columbia & 364 & 63.0 & 1 & 1.1 & 365 & 54.4 & 326 & 55.6 & 1 & 1.1 & 327 & 48.0 \\
\hline Florida & 4,672 & 27.0 & 15 & 0.5 & 4,687 & 23.2 & 4,940 & 28.0 & 17 & 0.6 & 4,957 & 24.0 \\
\hline Georgia & 2,585 & 30.7 & 7 & 0.4 & 2,592 & 25.4 & 2,709 & 31.8 & 7 & 0.4 & 2,716 & 26.3 \\
\hline Hawaii & 121 & 10.1 & 0 & 0.0 & 121 & 8.5 & 82 & 6.8 & 0 & 0.0 & 82 & 5.7 \\
\hline Idaho & 40 & 3.0 & 1 & 0.3 & 41 & 2.5 & 44 & 3.2 & 0 & 0.0 & 44 & 2.6 \\
\hline Illinois & 1,525 & 14.2 & 6 & 0.3 & 1,531 & 11.9 & 1,384 & 12.9 & 7 & 0.3 & 1,391 & 10.9 \\
\hline Indiana & 633 & 11.5 & 4 & 0.4 & 637 & 9.6 & 483 & 8.8 & 1 & 0.1 & 484 & 7.3 \\
\hline lowa & 124 & 4.8 & 0 & 0.0 & 124 & 4.0 & 133 & 5.1 & 4 & 0.8 & 137 & 4.4 \\
\hline Kansas & 154 & 6.5 & 2 & 0.4 & 156 & 5.4 & 141 & 5.9 & 1 & 0.2 & 142 & 4.9 \\
\hline Kentucky & 341 & 9.2 & 1 & 0.1 & 342 & 7.7 & 319 & 8.6 & 2 & 0.3 & 321 & 7.2 \\
\hline Louisiana & 1,112 & 28.8 & 2 & 0.2 & 1,114 & 23.9 & 1,151 & 29.7 & 2 & 0.2 & 1,153 & 24.6 \\
\hline Maine & 46 & 4.0 & 1 & 0.6 & 47 & 3.5 & 50 & 4.3 & 0 & 0.0 & 50 & 3.8 \\
\hline Maryland & 1,193 & 23.7 & 1 & 0.1 & 1,194 & 19.9 & 1,097 & 21.7 & 4 & 0.4 & 1,101 & 18.3 \\
\hline Massachusetts & 612 & 10.5 & 4 & 0.4 & 616 & 9.1 & 710 & 12.1 & 1 & 0.1 & 711 & 10.4 \\
\hline Michigan & 726 & 8.7 & 1 & 0.1 & 727 & 7.3 & 747 & 8.9 & 1 & 0.1 & 748 & 7.5 \\
\hline Minnesota & 294 & 6.5 & 2 & 0.2 & 296 & 5.4 & 286 & 6.2 & 3 & 0.3 & 289 & 5.2 \\
\hline Mississippi & 507 & 20.5 & 1 & 0.2 & 508 & 17.0 & 424 & 17.1 & 1 & 0.2 & 425 & 14.2 \\
\hline Missouri & 464 & 9.1 & 3 & 0.3 & 467 & 7.7 & 511 & 10.0 & 7 & 0.7 & 518 & 8.5 \\
\hline Montana & 19 & 2.2 & 0 & 0.0 & 19 & 1.8 & 17 & 1.9 & 1 & 0.6 & 18 & 1.7 \\
\hline Nebraska & 81 & 5.2 & 0 & 0.0 & 81 & 4.3 & 76 & 4.9 & 0 & 0.0 & 76 & 4.0 \\
\hline Nevada & 480 & 20.0 & 2 & 0.4 & 482 & 16.7 & 525 & 21.4 & 0 & 0.0 & 525 & 17.9 \\
\hline New Hampshire & 24 & 2.1 & 0 & 0.0 & 24 & 1.8 & 42 & 3.6 & 0 & 0.0 & 42 & 3.1 \\
\hline New Jersey & 1,161 & 15.4 & 5 & 0.4 & 1,166 & 13.0 & 1,143 & 15.2 & 3 & 0.2 & 1,146 & 12.8 \\
\hline New Mexico & 137 & 7.9 & 0 & 0.0 & 137 & 6.6 & 125 & 7.2 & 0 & 0.0 & 125 & 6.0 \\
\hline New York & 3,108 & 18.6 & 5 & 0.2 & 3,113 & 15.8 & 2,875 & $\begin{array}{l}17.2 \\
17.2\end{array}$ & 2 & 0.1 & 2,877 & 14.6 \\
\hline North Carolina & 1,331 & 15.8 & 9 & 0.6 & 1,340 & 13.4 & 1,404 & 16.5 & 10 & 0.6 & 1,414 & 13.9 \\
\hline North Dakota & 20 & 3.2 & 0 & 0.0 & 20 & 2.6 & 46 & 7.4 & 0 & 0.0 & 46 & 6.1 \\
\hline Ohio & 931 & 9.6 & 6 & 0.3 & 937 & 8.1 & 969 & 9.9 & 4 & 0.2 & 973 & 8.4 \\
\hline Oklahoma & 318 & 9.9 & 0 & 0.0 & 318 & 8.1 & 293 & 9.1 & 1 & 0.1 & 294 & 7.5 \\
\hline Oregon & 218 & 6.4 & 0 & 0.0 & 218 & 5.4 & 221 & 6.4 & 1 & 0.2 & 222 & 5.4 \\
\hline Pennsylvania & 1,177 & 10.8 & 6 & 0.3 & 1,183 & 9.2 & 1,150 & 10.6 & 2 & 0.1 & 1,152 & 9.0 \\
\hline Rhode Island & 64 & 7.0 & 0 & 0.0 & 64 & 6.1 & 70 & 7.7 & 0 & 0.0 & 70 & 6.6 \\
\hline South Carolina & 668 & 16.2 & 2 & 0.3 & 670 & 13.7 & 757 & 18.1 & 2 & 0.3 & 759 & 15.3 \\
\hline South Dakota & 23 & 3.3 & 2 & 1.3 & 25 & 2.9 & 39 & 5.5 & 1 & 0.6 & 40 & 4.6 \\
\hline Tennessee & 740 & 13.4 & 8 & 0.7 & 748 & 11.3 & 715 & 12.8 & 7 & 0.7 & 722 & 10.9 \\
\hline Texas & 4,479 & 20.2 & 16 & 0.3 & 4,495 & 16.4 & 4,464 & 19.8 & 8 & 0.2 & 4,472 & 16.1 \\
\hline Utah & 126 & 5.4 & 0 & 0.0 & 126 & 4.2 & 135 & 5.7 & 0 & 0.0 & 135 & 4.4 \\
\hline Vermont & 13 & 2.4 & 1 & 1.2 & 14 & 2.2 & 8 & 1.5 & 0 & 0.0 & 8 & 1.3 \\
\hline Virginia & 962 & $\begin{array}{l}2.7 \\
13.7\end{array}$ & 1 & 0.1 & 963 & 11.5 & 893 & 12.6 & 3 & 0.2 & 896 & 10.7 \\
\hline Washington & 450 & 7.5 & 4 & 0.3 & 454 & 6.3 & 432 & 7.1 & 3 & 0.3 & 435 & 6.0 \\
\hline West Virginia & 72 & 4.6 & 1 & 0.4 & 73 & 4.0 & 66 & 4.2 & 0 & 0.0 & 66 & 3.6 \\
\hline Wisconsin & 223 & 4.6 & 0 & 0.0 & 223 & 3.9 & 224 & 4.6 & 0 & 0.0 & 224 & 3.9 \\
\hline Wyoming & 17 & 3.5 & 0 & 0.0 & 17 & 2.9 & 20 & 4.1 & 0 & 0.0 & 20 & 3.4 \\
\hline Subtotal & 39,741 & 14.8 & 135 & 0.3 & 39,876 & 12.4 & 39,660 & 14.7 & 122 & 0.2 & 39,782 & 12.3 \\
\hline \multicolumn{13}{|l|}{ U.S. dependent areas } \\
\hline American Samoa & 0 & 0.0 & 0 & 0.0 & 0 & 0.0 & 0 & 0.0 & 0 & 0.0 & 0 & 0.0 \\
\hline Guam & 6 & 4.7 & 0 & 0.0 & 6 & 3.7 & 5 & 3.9 & 0 & 0.0 & 5 & 3.1 \\
\hline Northern Mariana Islands & 3 & 7.5 & 0 & 0.0 & 3 & 5.7 & 0 & 0.0 & 0 & 0.0 & 0 & 0.0 \\
\hline Puerto Rico & 544 & 18.3 & 0 & 0.0 & 544 & 15.7 & 523 & 17.8 & 0 & 0.0 & 523 & 15.3 \\
\hline Republic of Palau & 1 & 5.7 & 0 & 0.0 & 1 & 4.7 & 2 & 11.2 & 0 & 0.0 & 2 & 9.4 \\
\hline U.S. Virgin Islands & 12 & 13.7 & 0 & 0.0 & 12 & 11.6 & 12 & 13.7 & 0 & 0.0 & 12 & 11.7 \\
\hline Subtotal & 566 & 17.2 & 0 & 0.0 & 566 & 14.6 & 542 & 16.6 & 0 & 0.0 & 542 & 14.2 \\
\hline Total & 40,307 & 14.8 & 135 & 0.3 & 40,442 & 12.5 & 40,202 & 14.7 & 122 & 0.2 & 40,324 & 12.3 \\
\hline
\end{tabular}

Note. Data are based on address of residence at the end of the specified year (i.e., most recent known address). Data for the year 2016 are preliminary (subject to change) because they are based on only a 6 -month reporting delay. Numbers less than 12 , and rates and trends based on these numbers, should be interpreted with caution.

${ }^{a}$ Rates are per 100,000 population. 


\begin{tabular}{|c|c|c|c|c|c|c|c|c|c|c|c|c|c|c|c|}
\hline \multirow[b]{3}{*}{ Area of residence } & \multicolumn{6}{|c|}{2015} & \multicolumn{6}{|c|}{2016} & \multirow{2}{*}{\multicolumn{3}{|c|}{ Cumulative $^{a}$}} \\
\hline & \multicolumn{2}{|c|}{$\begin{array}{l}\text { Adults and } \\
\text { adolescents }\end{array}$} & \multicolumn{2}{|c|}{ Children } & \multicolumn{2}{|c|}{ Total } & \multicolumn{2}{|c|}{$\begin{array}{l}\text { Adults and } \\
\text { adolescents }\end{array}$} & \multicolumn{2}{|c|}{ Children } & \multicolumn{2}{|c|}{ Total } & & & \\
\hline & No. & Rate $^{b}$ & No. & Rate $^{b}$ & No. & Rate $^{b}$ & No. & Rate $^{b}$ & No. & Rate $^{b}$ & No. & Rate $^{b}$ & $\begin{array}{l}\text { Adults and } \\
\text { adolescents }\end{array}$ & Children & Total \\
\hline Alabama & 346 & 8.5 & 0 & 0.0 & 346 & 7.1 & 268 & 6.6 & 1 & 0.1 & 269 & 5.5 & 12,253 & 80 & 12,333 \\
\hline Alaska & 17 & 2.8 & 0 & 0.0 & 17 & 2.3 & 20 & 3.3 & 0 & 0.0 & 20 & 2.7 & 850 & 7 & 857 \\
\hline Arizona & 286 & 5.1 & 1 & 0.1 & 287 & 4.2 & 296 & 5.1 & 0 & 0.0 & 296 & 4.3 & 14,272 & 49 & 14,321 \\
\hline Arkansas & 130 & 5.3 & 1 & 0.2 & 131 & 4.4 & 110 & 4.4 & 0 & 0.0 & 110 & 3.7 & 5,304 & 38 & 5,342 \\
\hline California & 1,893 & 5.8 & 1 & 0.0 & 1,894 & 4.9 & 1,943 & 5.9 & 5 & 0.1 & 1,948 & 5.0 & 175,060 & 712 & 175,772 \\
\hline Colorado & 191 & 4.2 & 0 & 0.0 & 191 & 3.5 & 176 & 3.8 & 0 & 0.0 & 176 & 3.2 & 11,168 & 34 & 11,202 \\
\hline Connecticut & 139 & 4.5 & 0 & 0.0 & 139 & 3.9 & 135 & 4.4 & 0 & 0.0 & 135 & 3.8 & 16,865 & 184 & 17,049 \\
\hline Delaware & 61 & 7.6 & 0 & 0.0 & 61 & 6.5 & 70 & 8.7 & 0 & 0.0 & 70 & 7.4 & 4,544 & 29 & 4,573 \\
\hline District of Columbia & 180 & 31.1 & 0 & 0.0 & 180 & 26.9 & 185 & 31.6 & 0 & 0.0 & 185 & 27.2 & 21,242 & 194 & 21,436 \\
\hline Florida & 2,193 & 12.7 & 4 & 0.1 & 2,197 & 10.9 & 2,351 & 13.3 & 3 & 0.1 & 2,354 & 11.4 & 135,755 & 1,569 & 137,324 \\
\hline Georgia & 1,201 & 14.3 & 3 & 0.2 & 1,204 & 11.8 & 1,155 & 13.6 & 4 & 0.2 & 1,159 & 11.2 & 50,074 & 263 & 50,337 \\
\hline Hawaii & 42 & 3.5 & 0 & 0.0 & 42 & 2.9 & 33 & 2.7 & 0 & 0.0 & 33 & 2.3 & 3,577 & 18 & 3,595 \\
\hline Idaho & 23 & 1.7 & 1 & 0.3 & 24 & 1.5 & 22 & 1.6 & 0 & 0.0 & 22 & 1.3 & 866 & 4 & 870 \\
\hline Illinois & 638 & 5.9 & 1 & 0.0 & 639 & 5.0 & 614 & 5.7 & 3 & 0.1 & 617 & 4.8 & 43,530 & 286 & 43,816 \\
\hline Indiana & 256 & 4.7 & 2 & 0.2 & 258 & 3.9 & 229 & 4.2 & 0 & 0.0 & 229 & 3.5 & 11,295 & 62 & 11,357 \\
\hline lowa & 68 & 2.6 & 0 & 0.0 & 68 & 2.2 & 62 & 2.4 & 1 & 0.2 & 63 & 2.0 & 2,456 & 15 & 2,471 \\
\hline Kansas & 59 & 2.5 & 1 & 0.2 & 60 & 2.1 & 59 & 2.5 & 0 & 0.0 & 59 & 2.0 & 3,630 & 17 & 3,647 \\
\hline Kentucky & 142 & 3.8 & 0 & 0.0 & 142 & 3.2 & 151 & 4.1 & 0 & 0.0 & 151 & 3.4 & 6,586 & 39 & 6,625 \\
\hline Louisiana & 516 & 13.4 & 1 & 0.1 & 517 & 11.1 & 563 & 14.5 & 1 & 0.1 & 564 & 12.0 & 24,870 & 147 & 25,017 \\
\hline Maine & 25 & 2.2 & 0 & 0.0 & 25 & 1.9 & 17 & 1.5 & 0 & 0.0 & 17 & 1.3 & 1,417 & 9 & 1,426 \\
\hline Maryland & 632 & 12.6 & 0 & 0.0 & 632 & 10.5 & 585 & 11.6 & 1 & 0.1 & 586 & 9.7 & 40,172 & 341 & 40,513 \\
\hline Massachusetts & 275 & 4.7 & 2 & 0.2 & 277 & 4.1 & 243 & 4.2 & 0 & 0.0 & 243 & 3.6 & 24,441 & 232 & 24,673 \\
\hline Michigan & 352 & 4.2 & 1 & 0.1 & 353 & 3.6 & 375 & 4.5 & 1 & 0.1 & 376 & 3.8 & 19,794 & 121 & 19,915 \\
\hline Minnesota & 144 & 3.2 & 0 & 0.0 & 144 & 2.6 & 131 & 2.9 & 0 & 0.0 & 131 & 2.4 & 6,813 & 33 & 6,846 \\
\hline Mississippi & 270 & 10.9 & 0 & 0.0 & 270 & 9.0 & 276 & 11.2 & 0 & 0.0 & 276 & 9.2 & 9,804 & 58 & 9,862 \\
\hline Missouri & 223 & 4.4 & 1 & 0.1 & 224 & 3.7 & 225 & 4.4 & 2 & 0.2 & 227 & 3.7 & 14,233 & 68 & 14,301 \\
\hline Montana & 11 & 1.3 & 0 & 0.0 & 11 & 1.1 & 13 & 1.5 & 0 & 0.0 & 13 & 1.2 & 549 & 3 & 552 \\
\hline Nebraska & 47 & 3.0 & 0 & 0.0 & 47 & 2.5 & 35 & 2.2 & 0 & 0.0 & 35 & 1.8 & 2,080 & 12 & 2,092 \\
\hline Nevada & 206 & 8.6 & 0 & 0.0 & 206 & 7.1 & 239 & 9.8 & 0 & 0.0 & 239 & 8.1 & 8,114 & 29 & 8,143 \\
\hline New Hampshire & 15 & 1.3 & 0 & 0.0 & 15 & 1.1 & 21 & 1.8 & 0 & 0.0 & 21 & 1.6 & 1,348 & 10 & 1,358 \\
\hline New Jersey & 639 & 8.5 & 4 & 0.3 & 643 & 7.2 & 518 & 6.9 & 1 & 0.1 & 519 & 5.8 & 58,447 & 810 & 59,257 \\
\hline New Mexico & 55 & 3.2 & 0 & 0.0 & 55 & 2.6 & 60 & 3.5 & 1 & 0.3 & 61 & 2.9 & 3,494 & 10 & 3,504 \\
\hline New York & 1,544 & 9.2 & 2 & 0.1 & 1,546 & 7.8 & 1,572 & 9.4 & 3 & 0.1 & 1,575 & 8.0 & 201,388 & 2,430 & 203,818 \\
\hline North Carolina & 726 & 8.6 & 2 & 0.1 & 728 & 7.3 & 591 & 6.9 & 1 & 0.1 & 592 & 5.8 & 24,539 & 143 & 24,682 \\
\hline North Dakota & 14 & 2.2 & 0 & 0.0 & 14 & 1.8 & 15 & 2.4 & 0 & 0.0 & 15 & 2.0 & 236 & 2 & 238 \\
\hline
\end{tabular}




\begin{tabular}{|c|c|c|c|c|c|c|c|c|c|c|c|c|c|c|c|}
\hline \multirow[b]{3}{*}{ Area of residence } & \multicolumn{6}{|c|}{2015} & \multicolumn{6}{|c|}{2016} & \multirow{2}{*}{\multicolumn{3}{|c|}{ Cumulative $^{a}$}} \\
\hline & \multicolumn{2}{|c|}{$\begin{array}{l}\text { Adults and } \\
\text { adolescents }\end{array}$} & \multicolumn{2}{|c|}{ Children } & \multicolumn{2}{|c|}{ Total } & \multicolumn{2}{|c|}{$\begin{array}{l}\text { Adults and } \\
\text { adolescents }\end{array}$} & \multicolumn{2}{|c|}{ Children } & \multicolumn{2}{|c|}{ Total } & & & \\
\hline & No. & Rate $^{b}$ & No. & Rate $^{b}$ & No. & Rate $^{b}$ & No. & Rate $^{b}$ & No. & Rate $^{b}$ & No. & Rate $^{b}$ & $\begin{array}{l}\text { Adults and } \\
\text { adolescents }\end{array}$ & Children & Total \\
\hline Ohio & 404 & 4.1 & 2 & 0.1 & 406 & 3.5 & 484 & 5.0 & 1 & 0.1 & 485 & 4.2 & 20,856 & 154 & 21,010 \\
\hline Oklahoma & 153 & 4.8 & 0 & 0.0 & 153 & 3.9 & 148 & 4.6 & 1 & 0.1 & 149 & 3.8 & 6,539 & 27 & 6,566 \\
\hline Oregon & 122 & 3.6 & 0 & 0.0 & 122 & 3.0 & 94 & 2.7 & 0 & 0.0 & 94 & 2.3 & 7,647 & 19 & 7,666 \\
\hline Pennsylvania & 612 & 5.6 & 2 & 0.1 & 614 & 4.8 & 538 & 4.9 & 1 & 0.1 & 539 & 4.2 & 42,470 & 374 & 42,844 \\
\hline Rhode Island & 33 & 3.6 & 0 & 0.0 & 33 & 3.1 & 30 & 3.3 & 0 & 0.0 & 30 & 2.8 & 3,194 & 29 & 3,223 \\
\hline South Carolina & 385 & 9.4 & 1 & 0.1 & 386 & 7.9 & 368 & 8.8 & 1 & 0.1 & 369 & 7.4 & 18,799 & 120 & 18,919 \\
\hline South Dakota & 14 & 2.0 & 0 & 0.0 & 14 & 1.6 & 21 & 3.0 & 0 & 0.0 & 21 & 2.4 & 401 & 7 & 408 \\
\hline Tennessee & 336 & 6.1 & 3 & 0.3 & 339 & 5.1 & 297 & 5.3 & 0 & 0.0 & 297 & 4.5 & 17,452 & 70 & 17,522 \\
\hline Texas & 2,047 & 9.2 & 2 & 0.0 & 2,049 & 7.5 & 2,073 & 9.2 & 4 & 0.1 & 2,077 & 7.5 & 95,506 & 411 & 95,917 \\
\hline Utah & 54 & 2.3 & 0 & 0.0 & 54 & 1.8 & 42 & 1.8 & 0 & 0.0 & 42 & 1.4 & 2,924 & 21 & 2,945 \\
\hline Vermont & 11 & 2.0 & 0 & 0.0 & 11 & 1.8 & 9 & 1.7 & 0 & 0.0 & 9 & 1.4 & 575 & 6 & 581 \\
\hline Virginia & 372 & 5.3 & 1 & 0.1 & 373 & 4.5 & 332 & 4.7 & 1 & 0.1 & 333 & 4.0 & 22,317 & 192 & 22,509 \\
\hline Washington & 197 & 3.3 & 0 & 0.0 & 197 & 2.8 & 174 & 2.9 & 1 & 0.1 & 175 & 2.4 & 14,672 & 34 & 14,706 \\
\hline West Virginia & 34 & 2.2 & 0 & 0.0 & 34 & 1.8 & 43 & 2.7 & 0 & 0.0 & 43 & 2.3 & 2,035 & 11 & 2,046 \\
\hline Wisconsin & 101 & 2.1 & 1 & 0.1 & 102 & 1.8 & 101 & 2.1 & 0 & 0.0 & 101 & 1.7 & 5,977 & 38 & 6,015 \\
\hline Wyoming & 6 & 1.2 & 0 & 0.0 & 6 & 1.0 & 10 & 2.1 & 0 & 0.0 & 10 & 1.7 & 343 & 2 & 345 \\
\hline Subtotal & 18,440 & 6.9 & 40 & 0.1 & 18,480 & 5.8 & 18,122 & 6.7 & 38 & 0.1 & 18,160 & 5.6 & $1,222,773$ & 9,573 & $1,232,346$ \\
\hline \multicolumn{16}{|l|}{ U.S. dependent areas } \\
\hline American Samoa & 0 & 0.0 & 0 & 0.0 & 0 & 0.0 & 0 & 0.0 & 0 & 0.0 & 0 & 0.0 & 1 & 0 & 1 \\
\hline Guam & 2 & 1.6 & 0 & 0.0 & 2 & 1.2 & 5 & 3.9 & 0 & 0.0 & 5 & 3.1 & 91 & 2 & 93 \\
\hline Northern Mariana Islands & 2 & 5.0 & 0 & 0.0 & 2 & 3.8 & 0 & 0.0 & 0 & 0.0 & 0 & 0.0 & 5 & 0 & 5 \\
\hline Puerto Rico & 244 & 8.2 & 0 & 0.0 & 244 & 7.0 & 236 & 8.0 & 0 & 0.0 & 236 & 6.9 & 34,919 & 404 & 35,323 \\
\hline Republic of Palau & 0 & 0.0 & 0 & 0.0 & 0 & 0.0 & 1 & 5.6 & 0 & 0.0 & 1 & 4.7 & 6 & 0 & 6 \\
\hline U.S. Virgin Islands & 10 & 11.4 & 0 & 0.0 & 10 & 9.7 & 6 & 6.8 & 1 & 6.5 & 7 & 6.8 & 800 & 21 & 821 \\
\hline Subtotal & 258 & 7.8 & 0 & 0.0 & 258 & 6.7 & 248 & 7.6 & 1 & 0.2 & 249 & 6.5 & 35,822 & 427 & 36,249 \\
\hline Total & 18,698 & 6.9 & 40 & 0.1 & 18,738 & 5.8 & 18,370 & 6.7 & 39 & 0.1 & 18,409 & 5.6 & $1,258,595$ & 10,000 & $1,268,595$ \\
\hline
\end{tabular}


than 12 , and rates and trends based on these numbers, should be interpreted with caution.

$a$ From the beginning of the epidemic through 2016.

${ }^{b}$ Rates are per 100,000 population. 


\begin{tabular}{|c|c|c|c|c|c|c|c|c|c|c|c|c|c|c|c|c|}
\hline \multirow[b]{2}{*}{ Area of residence } & \multicolumn{2}{|c|}{$\begin{array}{c}\text { American Indian/ } \\
\text { Alaska Native }\end{array}$} & \multicolumn{2}{|c|}{ Asian $^{a}$} & \multicolumn{2}{|c|}{$\begin{array}{c}\text { Black/African } \\
\text { American }\end{array}$} & \multicolumn{2}{|c|}{ Hispanic/Latino ${ }^{b}$} & \multicolumn{2}{|c|}{$\begin{array}{l}\text { Native Hawaiian/ } \\
\text { Other Pacific } \\
\text { Islander }\end{array}$} & \multicolumn{2}{|c|}{ White } & \multicolumn{2}{|c|}{ Multiple races } & \multicolumn{2}{|c|}{ Total $^{\mathrm{C}}$} \\
\hline & No. & Rate $^{d}$ & No. & Rate $^{d}$ & No. & Rate $^{d}$ & No. & Rate $^{d}$ & No. & Rate $^{d}$ & No. & Rate $^{d}$ & No. & Rate $^{d}$ & No. & Rate $^{d}$ \\
\hline Alabama & 5 & 21.3 & 32 & 58.9 & 7,883 & 747.4 & 370 & 267.7 & 5 & 270.1 & 3,391 & 123.1 & 584 & $1,317.5$ & 12,316 & 302.4 \\
\hline Alaska & 156 & 190.6 & 19 & 50.9 & 87 & 430.2 & 62 & 167.7 & 3 & 44.7 & 294 & 76.0 & 36 & 115.9 & 657 & 109.3 \\
\hline Arizona & 579 & 267.3 & 178 & 99.7 & 1,880 & 825.3 & 4,492 & 284.0 & 21 & 205.6 & 7,660 & 228.6 & 406 & 471.5 & 15,261 & 270.0 \\
\hline Arkansas & 5 & 27.3 & 16 & 44.0 & 2,326 & 631.3 & 337 & 224.6 & 5 & 92.2 & 2,366 & 127.3 & 249 & 743.5 & 5,308 & 214.8 \\
\hline California & 331 & 235.1 & 4,726 & 100.1 & 20,804 & $1,106.7$ & 43,727 & 374.8 & 245 & 205.7 & 48,798 & 370.0 & 3,437 & 480.5 & 122,079 & 376.4 \\
\hline Colorado & 61 & 205.9 & 118 & 85.1 & 1,672 & 947.9 & 2,462 & 282.9 & 13 & 220.8 & 6,914 & 213.3 & 226 & 289.1 & 11,519 & 253.6 \\
\hline Connecticut & 11 & 179.8 & 92 & 69.1 & 3,391 & $1,140.8$ & 3,514 & 829.9 & 4 & 377.0 & 3,140 & 145.4 & 207 & 536.6 & 10,360 & 338.7 \\
\hline Delaware & 3 & 116.4 & 7 & 23.0 & 1,877 & $1,133.4$ & 274 & 446.3 & 1 & 362.3 & 954 & 181.8 & 114 & 910.6 & 3,230 & 404.9 \\
\hline District of Columbia & 13 & $1,018.8$ & 73 & 306.7 & 10,853 & $4,113.7$ & 1,107 & $1,954.1$ & 8 & $2,730.4$ & 2,431 & $1,098.5$ & 480 & $4,411.4$ & 14,972 & $2,590.2$ \\
\hline Florida & 58 & 126.7 & 480 & 102.4 & 49,338 & $1,929.8$ & 24,209 & 595.2 & 40 & 375.6 & 30,134 & 302.4 & 2,322 & $1,112.7$ & 106,585 & 615.2 \\
\hline Georgia & 20 & 100.9 & 210 & 64.0 & 33,946 & $1,323.5$ & 3,318 & 488.8 & 14 & 282.6 & 9,556 & 203.0 & 2,387 & $2,219.3$ & 49,463 & 588.0 \\
\hline Hawaii & 9 & 330.0 & 413 & 87.9 & 162 & 719.4 & 322 & 315.4 & 250 & 228.6 & 1,369 & 480.3 & 263 & 128.6 & 2,788 & 233.1 \\
\hline Idaho & 11 & 73.5 & 7 & 37.9 & 88 & $1,036.6$ & 162 & 113.4 & 0 & 0.0 & 768 & 67.7 & 26 & 126.9 & 1,062 & 79.2 \\
\hline Illinois & 33 & 199.8 & 436 & 76.1 & 16,508 & $1,101.9$ & 6,629 & 405.7 & 21 & 727.7 & 9,963 & 144.6 & 1,849 & $1,527.8$ & 35,441 & 330.1 \\
\hline Indiana & 5 & 38.5 & 169 & 148.0 & 3,817 & 780.6 & 960 & 306.6 & 0 & 0.0 & 5,410 & 120.5 & 380 & 564.2 & 10,741 & 195.7 \\
\hline lowa & 4 & 54.9 & 58 & 96.0 & 458 & 574.0 & 224 & 180.6 & 0 & 0.0 & 1,565 & 68.2 & 118 & 423.9 & 2,427 & 93.5 \\
\hline Kansas & 8 & 40.9 & 34 & 50.2 & 704 & 519.5 & 487 & 205.8 & 1 & 55.3 & 1,441 & 76.6 & 152 & 346.3 & 2,830 & 118.6 \\
\hline Kentucky & 6 & 74.6 & 41 & 81.7 & 2,173 & 753.0 & 422 & 399.9 & 1 & 50.3 & 3,745 & 117.0 & 254 & 586.4 & 6,644 & 179.6 \\
\hline Louisiana & 31 & 126.2 & 67 & 98.4 & 13,302 & $1,107.4$ & 875 & 500.0 & 6 & 367.9 & 4,876 & 207.3 & 331 & 845.3 & 19,492 & 504.7 \\
\hline Maine & 6 & 84.9 & 6 & 44.9 & 194 & $1,458.4$ & 97 & 625.4 & 1 & 314.5 & 1,129 & 103.9 & 44 & 316.9 & 1,478 & 128.5 \\
\hline Maryland & 18 & 144.8 & 205 & 64.3 & 24,240 & $1,645.9$ & 2,160 & 511.7 & 4 & 161.0 & 4,424 & 163.1 & 2,020 & $2,340.3$ & 33,072 & 657.8 \\
\hline Massachusetts & 24 & 233.3 & 379 & 103.4 & 5,690 & $1,474.1$ & 5,221 & 899.8 & 4 & 175.7 & 7,980 & 181.8 & 364 & 480.8 & 19,665 & 338.4 \\
\hline Michigan & 26 & 55.3 & 109 & 45.0 & 8,294 & 736.4 & 811 & 230.6 & 2 & 94.1 & 4,869 & 75.3 & 500 & 378.2 & 14,615 & 174.6 \\
\hline Minnesota & 104 & 232.0 & 141 & 69.1 & 2,710 & $1,141.7$ & 778 & 392.4 & 5 & 255.2 & 3,812 & 100.3 & 248 & 362.3 & 7,803 & 171.3 \\
\hline Mississippi & 11 & 96.3 & 17 & 66.3 & 6,726 & 750.8 & 295 & 451.7 & 1 & 114.9 & 1,778 & 122.5 & 374 & $1,985.8$ & 9,236 & 374.0 \\
\hline Missouri & 14 & 63.8 & 72 & 74.0 & 5,202 & 913.8 & 636 & 361.9 & 5 & 91.1 & 5,615 & 135.7 & 341 & 456.6 & 11,887 & 234.0 \\
\hline Montana & 26 & 55.5 & 1 & 14.9 & 18 & 431.1 & 35 & 132.4 & 2 & 322.6 & 462 & 60.3 & 30 & 180.2 & 574 & 66.1 \\
\hline Nebraska & 22 & 186.5 & 31 & 88.4 & 569 & 836.7 & 306 & 223.7 & 1 & 108.5 & 1,059 & 82.8 & 51 & 269.8 & 2,040 & 131.6 \\
\hline Nevada & 50 & 238.1 & 271 & 132.0 & 2,118 & $1,068.0$ & 2,200 & 359.3 & 33 & 231.5 & 3,968 & 307.7 & 264 & 441.2 & 8,906 & 371.0 \\
\hline New Hampshire & 0 & 0.0 & 11 & 38.6 & 143 & $1,089.5$ & 165 & 485.0 & 0 & 0.0 & 871 & 82.4 & 45 & 351.1 & 1,236 & 107.6 \\
\hline New Jersey & 11 & 103.0 & 310 & 44.0 & 16,134 & $1,674.2$ & 9,939 & 723.6 & 9 & 372.1 & 6,927 & 158.0 & 2,248 & $2,665.6$ & 35,636 & 473.7 \\
\hline New Mexico & 242 & 167.6 & 14 & 53.7 & 189 & 597.1 & 1,522 & 193.3 & 1 & 87.6 & 1,154 & 162.1 & 92 & 434.0 & 3,215 & 186.5 \\
\hline New York & 59 & 122.1 & 1,584 & 110.5 & 46,171 & $1,908.8$ & 51,196 & $1,731.1$ & 16 & 224.8 & 21,438 & 222.0 & 7,752 & $3,576.5$ & 128,681 & 768.8 \\
\hline North Carolina & 178 & 188.8 & 149 & 67.2 & 18,596 & $1,045.7$ & 2,285 & 360.4 & 10 & 199.0 & 7,498 & 134.9 & 1,090 & 996.3 & 29,814 & 354.9 \\
\hline North Dakota & 11 & 39.1 & 3 & 33.9 & 107 & 752.6 & 20 & 109.6 & 0 & 0.0 & 180 & 32.9 & 13 & 153.6 & 334 & 53.4 \\
\hline
\end{tabular}




\begin{tabular}{|c|c|c|c|c|c|c|c|c|c|c|c|c|c|c|c|c|}
\hline \multirow[b]{2}{*}{ Area of residence } & \multicolumn{2}{|c|}{$\begin{array}{l}\text { American Indian/ } \\
\text { Alaska Native }\end{array}$} & \multicolumn{2}{|c|}{ Asian $^{a}$} & \multicolumn{2}{|c|}{$\begin{array}{c}\text { Black/African } \\
\text { American }\end{array}$} & \multicolumn{2}{|c|}{ Hispanic/Latino ${ }^{b}$} & \multicolumn{2}{|c|}{$\begin{array}{c}\text { Native Hawaiian/ } \\
\text { Other Pacific } \\
\text { Islander }\end{array}$} & \multicolumn{2}{|c|}{ White } & \multicolumn{2}{|c|}{ Multiple races } & \multicolumn{2}{|c|}{ Total $^{\mathrm{C}}$} \\
\hline & No. & Rate $^{d}$ & No. & Rate $^{d}$ & No. & Rate $^{d}$ & No. & Rate $^{d}$ & No. & Rate $^{d}$ & No. & Rate $^{d}$ & No. & Rate $^{d}$ & No. & Rate $^{d}$ \\
\hline Ohio & 17 & 88.4 & 94 & 46.9 & 9,072 & 783.1 & 1,255 & 422.0 & 2 & 61.7 & 9,357 & 118.0 & 801 & 584.1 & 20,709 & 212.5 \\
\hline Oklahoma & 293 & 114.3 & 67 & 97.3 & 1,350 & 578.2 & 547 & 199.9 & 8 & 198.0 & 3,204 & 143.7 & 305 & 212.9 & 5,774 & 179.9 \\
\hline Oregon & 47 & 124.6 & 123 & 84.2 & 453 & 761.6 & 869 & 236.1 & 19 & 151.5 & 4,885 & 181.2 & 202 & 234.0 & 6,598 & 193.7 \\
\hline Pennsylvania & 31 & 203.1 & 250 & 71.1 & 16,545 & $1,474.8$ & 5,616 & 870.6 & 22 & 707.9 & 10,278 & 119.1 & 1,487 & $1,224.2$ & 34,233 & 314.4 \\
\hline Rhode Island & 7 & 189.9 & 38 & 126.1 & 548 & $1,100.8$ & 627 & 540.9 & 1 & 174.5 & 1,077 & 155.1 & 59 & 424.0 & 2,357 & 259.5 \\
\hline South Carolina & 12 & 78.0 & 35 & 56.3 & 11,165 & $1,024.2$ & 752 & 393.3 & 3 & 135.6 & 3,886 & 143.7 & 361 & 790.3 & 16,224 & 394.6 \\
\hline South Dakota & 78 & 150.7 & 4 & 42.1 & 116 & $1,017.3$ & 30 & 146.2 & 0 & 0.0 & 274 & 45.8 & 12 & 119.0 & 514 & 73.3 \\
\hline Tennessee & 10 & 64.1 & 65 & 69.8 & 9,141 & $1,016.3$ & 820 & 353.4 & 7 & 260.2 & 5,872 & 139.3 & 507 & 782.0 & 16,425 & 297.4 \\
\hline Texas & 41 & 53.4 & 810 & 78.8 & 30,207 & $1,150.2$ & 26,087 & 324.3 & 25 & 139.5 & 21,626 & 212.8 & 3,069 & $1,279.1$ & 81,873 & 368.9 \\
\hline Utah & 23 & 100.8 & 56 & 95.4 & 241 & $1,028.3$ & 583 & 201.1 & 5 & 23.7 & 1,734 & 92.7 & 57 & 164.7 & 2,702 & 116.4 \\
\hline Vermont & 0 & 0.0 & 9 & 103.2 & 62 & $1,068.4$ & 55 & 604.5 & 1 & 609.8 & 519 & 101.9 & 25 & 337.0 & 671 & 123.7 \\
\hline Virginia & 16 & 83.5 & 258 & 58.2 & 12,597 & 949.6 & 1,902 & 338.0 & 8 & 160.7 & 6,147 & 135.5 & 645 & 498.7 & 21,607 & 307.7 \\
\hline Washington & 128 & 167.9 & 412 & 82.5 & 1,911 & 878.3 & 1,711 & 273.8 & 54 & 143.4 & 7,540 & 173.2 & 719 & 389.8 & 12,484 & 208.3 \\
\hline West Virginia & 1 & 29.5 & 7 & 54.8 & 396 & 729.2 & 81 & 391.4 & 0 & 0.0 & 1,197 & 81.9 & 95 & 541.4 & 1,781 & 113.3 \\
\hline Wisconsin & 31 & 76.6 & 78 & 63.9 & 2,177 & 772.0 & 817 & 304.6 & 1 & 67.1 & 2,648 & 64.9 & 163 & 305.4 & 5,916 & 122.0 \\
\hline Wyoming & 10 & 107.2 & 2 & 44.4 & 24 & 417.3 & 57 & 134.2 & 0 & 0.0 & 190 & 45.8 & 6 & 96.5 & 289 & 59.7 \\
\hline Subtotal & 2,896 & 150.8 & 12,787 & 87.3 & 404,375 & $1,238.3$ & 213,428 & 496.8 & 888 & 197.1 & 298,373 & 174.2 & 37,810 & 890.0 & 971,524 & 362.3 \\
\hline
\end{tabular}

\section{U.S. dependent areas}

\begin{tabular}{|c|c|c|c|c|c|c|c|c|c|c|c|c|c|c|c|c|}
\hline American Samoa & 0 & - & 0 & - & 0 & - & 0 & - & 1 & - & 0 & - & 1 & - & 2 & 4.7 \\
\hline Guam & 0 & - & 39 & - & 3 & - & 2 & - & 17 & - & 11 & - & 3 & - & 75 & 59.3 \\
\hline Northern Mariana Islands & 0 & - & 1 & - & 0 & - & 0 & - & 2 & - & 2 & - & 1 & - & 6 & 14.9 \\
\hline Puerto Rico & 1 & - & 1 & - & 3 & - & 16,757 & - & 1 & - & 19 & - & 1 & - & 16,783 & 564 \\
\hline Republic of Palau & 0 & - & 2 & - & 0 & - & 1 & - & 4 & - & 0 & - & 0 & - & 7 & 39.6 \\
\hline U.S. Virgin Islands & 0 & - & 2 & - & 311 & - & 196 & - & 0 & - & 42 & - & 4 & - & 558 & 635.5 \\
\hline Subtotal & 1 & - & 45 & - & 317 & - & 16,956 & - & 25 & - & 74 & - & 10 & - & 17,431 & 529.7 \\
\hline Total & 2,897 & - & 12,832 & - & 404,692 & - & 230,384 & - & 913 & - & 298,447 & - & 37,820 & - & 988,955 & 364.3 \\
\hline
\end{tabular}

Note. Data are based on address of residence at the end of the specified year (i.e., most recent known address). Numbers less than 12, and rates based on these numbers, should be interpreted with caution

a Includes Asian/Pacific Islander legacy cases (see Technical Notes).

${ }^{b}$ Hispanics/Latinos can be of any race.

${ }^{\mathrm{c}}$ Includes persons whose race/ethnicity is unknown.

d Rates are per 100,000 population.

${ }^{\mathrm{e}}$ Rates by race/ethnicity are not provided because U.S. census information for U.S. dependent areas is limited. 


\begin{tabular}{|c|c|c|c|c|c|c|c|c|c|c|c|c|c|c|c|c|}
\hline \multirow[b]{2}{*}{ Area of residence } & \multicolumn{2}{|c|}{$\begin{array}{l}\text { American Indian/ } \\
\text { Alaska Native }\end{array}$} & \multicolumn{2}{|c|}{ Asian $^{a}$} & \multicolumn{2}{|c|}{$\begin{array}{c}\text { Black/African } \\
\text { American }\end{array}$} & \multicolumn{2}{|c|}{ Hispanic/Latino ${ }^{b}$} & \multicolumn{2}{|c|}{$\begin{array}{l}\text { Native Hawaiian/ } \\
\text { Other Pacific } \\
\text { Islander }\end{array}$} & \multicolumn{2}{|c|}{ White } & \multicolumn{2}{|c|}{ Multiple races } & \multicolumn{2}{|c|}{ Total $^{\mathrm{C}}$} \\
\hline & No. & Rate $^{d}$ & No. & Rate $^{d}$ & No. & Rate $^{d}$ & No. & Rate $^{d}$ & No. & Rate $^{d}$ & No. & Rate $^{d}$ & No. & Rate $^{d}$ & No. & Rate $^{d}$ \\
\hline Alabama & 0 & 0.0 & 12 & 22.1 & 3,579 & 339.3 & 195 & 141.1 & 3 & 162.1 & 1,466 & 53.2 & 296 & 667.8 & 5,551 & 136.3 \\
\hline Alaska & 92 & 112.4 & 15 & 40.2 & 37 & 182.9 & 38 & 102.8 & 2 & 29.8 & 165 & 42.7 & 19 & 61.2 & 368 & 61.2 \\
\hline Arizona & 305 & 140.8 & 70 & 39.2 & 830 & 364.4 & 2,235 & 141.3 & 11 & 107.7 & 3,851 & 114.9 & 210 & 243.9 & 7,513 & 132.9 \\
\hline Arkansas & 2 & 10.9 & 6 & 16.5 & 1,007 & 273.3 & 172 & 114.6 & 3 & 55.3 & 1,117 & 60.1 & 135 & 403.1 & 2,442 & 98.8 \\
\hline California & 174 & 123.6 & 2,470 & 52.3 & 11,778 & 626.5 & 25,350 & 217.3 & 127 & 106.6 & 27,546 & 208.8 & 1,893 & 264.7 & 69,345 & 213.8 \\
\hline Colorado & 30 & 101.2 & 49 & 35.3 & 761 & 431.4 & 1,267 & 145.6 & 5 & 84.9 & 2,880 & 88.8 & 133 & 170.2 & 5,125 & 112.9 \\
\hline Connecticut & 7 & 114.4 & 46 & 34.6 & 2,118 & 712.6 & 2,264 & 534.7 & 3 & 282.8 & 1,910 & 88.5 & 133 & 344.8 & 6,481 & 211.9 \\
\hline Delaware & 2 & 77.6 & 5 & 16.4 & 1,188 & 717.3 & 166 & 270.4 & 1 & 362.3 & 555 & 105.7 & 64 & 511.2 & 1,981 & 248.3 \\
\hline District of Columbia & 7 & 548.6 & 33 & 138.7 & 6,138 & $2,326.6$ & 551 & 972.7 & 2 & 682.6 & 1,056 & 477.2 & 267 & $2,453.8$ & 8,055 & $1,393.6$ \\
\hline Florida & 25 & 54.6 & 207 & 44.2 & 28,269 & $1,105.7$ & 12,389 & 304.6 & 12 & 112.7 & 16,300 & 163.5 & 1,444 & 692.0 & 58,647 & 338.5 \\
\hline Georgia & 7 & 35.3 & 85 & 25.9 & 17,652 & 688.2 & 1,812 & 266.9 & 4 & 80.7 & 4,877 & 103.6 & 1,406 & $1,307.2$ & 25,843 & 307.2 \\
\hline Hawaii & 4 & 146.7 & 244 & 51.9 & 88 & 390.8 & 191 & 187.1 & 156 & 142.7 & 793 & 278.2 & 148 & 72.4 & 1,624 & 135.8 \\
\hline Idaho & 4 & 26.7 & 1 & 5.4 & 36 & 424.1 & 88 & 61.6 & 0 & 0.0 & 383 & 33.8 & 17 & 83.0 & 529 & 39.4 \\
\hline Illinois & 10 & 60.6 & 203 & 35.4 & 8,443 & 563.5 & 3,633 & 222.3 & 10 & 346.5 & 4,869 & 70.7 & 1,062 & 877.5 & 18,230 & 169.8 \\
\hline Indiana & 3 & 23.1 & 75 & 65.7 & 1,856 & 379.5 & 521 & 166.4 & 0 & 0.0 & 2,797 & 62.3 & 216 & 320.7 & 5,468 & 99.6 \\
\hline lowa & 3 & 41.2 & 24 & 39.7 & 245 & 307.1 & 137 & 110.5 & 0 & 0.0 & 899 & 39.2 & 60 & 215.5 & 1,368 & 52.7 \\
\hline Kansas & 2 & 10.2 & 13 & 19.2 & 335 & 247.2 & 281 & 118.8 & 0 & 0.0 & 783 & 41.6 & 89 & 202.8 & 1,503 & 63.0 \\
\hline Kentucky & 3 & 37.3 & 13 & 25.9 & 1,044 & 361.8 & 259 & 245.5 & 0 & 0.0 & 1,912 & 59.7 & 154 & 355.5 & 3,385 & 91.5 \\
\hline Louisiana & 14 & 57.0 & 28 & 41.1 & 6,951 & 578.7 & 473 & 270.3 & 2 & 122.6 & 2,563 & 108.9 & 180 & 459.7 & 10,212 & 264.4 \\
\hline Maine & 3 & 42.5 & 4 & 30.0 & 77 & 578.9 & 49 & 315.9 & 0 & 0.0 & 615 & 56.6 & 26 & 187.2 & 774 & 67.3 \\
\hline Maryland & 4 & 32.2 & 85 & 26.6 & 13,186 & 895.3 & 1,231 & 291.6 & 2 & 80.5 & 2,211 & 81.5 & 1,211 & $1,403.0$ & 17,930 & 356.6 \\
\hline Massachusetts & 14 & 136.1 & 199 & 54.3 & 3,376 & 874.6 & 3,071 & 529.2 & 1 & 43.9 & 4,364 & 99.4 & 206 & 272.1 & 11,233 & 193.3 \\
\hline Michigan & 11 & 23.4 & 43 & 17.7 & 4,331 & 384.5 & 438 & 124.5 & 2 & 94.1 & 2,565 & 39.7 & 288 & 217.8 & 7,678 & 91.7 \\
\hline Minnesota & 54 & 120.5 & 73 & 35.8 & 1,304 & 549.4 & 431 & 217.4 & 1 & 51.0 & 1,711 & 45.0 & 137 & 200.2 & 3,711 & 81.5 \\
\hline Mississippi & 2 & 17.5 & 8 & 31.2 & 3,222 & 359.6 & 164 & 251.1 & 0 & 0.0 & 868 & 59.8 & 222 & $1,178.7$ & 4,486 & 181.6 \\
\hline Missouri & 9 & 41.0 & 33 & 33.9 & 2,782 & 488.7 & 352 & 200.3 & 3 & 54.6 & 2,947 & 71.2 & 215 & 287.9 & 6,341 & 124.8 \\
\hline Montana & 18 & 38.4 & 0 & 0.0 & 12 & 287.4 & 16 & 60.5 & 1 & 161.3 & 270 & 35.2 & 22 & 132.2 & 339 & 39.0 \\
\hline Nebraska & 9 & 76.3 & 16 & 45.6 & 301 & 442.6 & 170 & 124.3 & 1 & 108.5 & 539 & 42.2 & 33 & 174.6 & 1,069 & 69.0 \\
\hline Nevada & 27 & 128.6 & 120 & 58.4 & 1,049 & 529.0 & 1,108 & 181.0 & 17 & 119.3 & 1,940 & 150.5 & 138 & 230.6 & 4,399 & 183.3 \\
\hline New Hampshire & 0 & 0.0 & 8 & 28.1 & 72 & 548.6 & 101 & 296.9 & 0 & 0.0 & 440 & 41.6 & 29 & 226.2 & 650 & 56.6 \\
\hline New Jersey & 6 & 56.2 & 146 & 20.7 & 8,431 & 874.9 & 5,284 & 384.7 & 2 & 82.7 & 3,492 & 79.7 & 1,396 & $1,655.3$ & 18,759 & 249.4 \\
\hline New Mexico & 128 & 88.7 & 6 & 23.0 & 92 & 290.6 & 844 & 107.2 & 1 & 87.6 & 718 & 100.8 & 54 & 254.7 & 1,843 & 106.9 \\
\hline New York & 32 & 66.2 & 752 & 52.5 & 27,006 & $1,116.5$ & 31,366 & $1,060.6$ & 4 & 56.2 & 11,049 & 114.4 & 4,929 & $2,274.0$ & 75,160 & 449.0 \\
\hline North Carolina & 76 & 80.6 & 45 & 20.3 & 8,377 & 471.0 & 1,097 & 173.0 & 2 & 39.8 & 3,251 & 58.5 & 562 & 513.7 & 13,410 & 159.6 \\
\hline North Dakota & 8 & 28.5 & 3 & 33.9 & 47 & 330.6 & 13 & 71.3 & 0 & 0.0 & 86 & 15.7 & 9 & 106.3 & 166 & 26.5 \\
\hline
\end{tabular}




\begin{tabular}{|c|c|c|c|c|c|c|c|c|c|c|c|c|c|c|c|c|}
\hline \multirow[b]{2}{*}{ Area of residence } & \multicolumn{2}{|c|}{$\begin{array}{l}\text { American Indian/ } \\
\text { Alaska Native }\end{array}$} & \multicolumn{2}{|c|}{ Asian $^{a}$} & \multicolumn{2}{|c|}{$\begin{array}{c}\text { Black/African } \\
\text { American }\end{array}$} & \multicolumn{2}{|c|}{ Hispanic/Latino ${ }^{b}$} & \multicolumn{2}{|c|}{$\begin{array}{c}\text { Native Hawaiian/ } \\
\text { Other Pacific } \\
\text { Islander }\end{array}$} & \multicolumn{2}{|c|}{ White } & \multicolumn{2}{|c|}{ Multiple races } & \multicolumn{2}{|c|}{ Total $^{\mathrm{C}}$} \\
\hline & No. & Rate $^{d}$ & No. & Rate $^{d}$ & No. & Rate $^{d}$ & No. & Rate $^{d}$ & No. & Rate $^{d}$ & No. & Rate $^{d}$ & No. & Rate $^{d}$ & No. & Rate $^{d}$ \\
\hline Ohio & 8 & 41.6 & 34 & 17.0 & 4,248 & 366.7 & 626 & 210.5 & 0 & 0.0 & 4,516 & 57.0 & 433 & 315.8 & 9,866 & 101.2 \\
\hline Oklahoma & 149 & 58.1 & 28 & 40.7 & 627 & 268.5 & 277 & 101.2 & 3 & 74.2 & 1,583 & 71.0 & 157 & 109.6 & 2,824 & 88.0 \\
\hline Oregon & 32 & 84.9 & 56 & 38.4 & 247 & 415.2 & 511 & 138.9 & 10 & 79.7 & 2,850 & 105.7 & 104 & 120.5 & 3,810 & 111.9 \\
\hline Pennsylvania & 12 & 78.6 & 122 & 34.7 & 9,226 & 822.4 & 3,127 & 484.7 & 9 & 289.6 & 5,600 & 64.9 & 892 & 734.3 & 18,988 & 174.4 \\
\hline Rhode Island & 3 & 81.4 & 23 & 76.3 & 331 & 664.9 & 397 & 342.5 & 1 & 174.5 & 612 & 88.2 & 23 & 165.3 & 1,390 & 153.0 \\
\hline South Carolina & 5 & 32.5 & 19 & 30.6 & 6,186 & 567.4 & 418 & 218.6 & 2 & 90.4 & 1,987 & 73.5 & 239 & 523.2 & 8,856 & 215.4 \\
\hline South Dakota & 39 & 75.3 & 1 & 10.5 & 47 & 412.2 & 17 & 82.9 & 0 & 0.0 & 109 & 18.2 & 7 & 69.4 & 220 & 31.4 \\
\hline Tennessee & 5 & 32.0 & 31 & 33.3 & 4,433 & 492.9 & 436 & 187.9 & 2 & 74.3 & 3,044 & 72.2 & 321 & 495.1 & 8,272 & 149.8 \\
\hline Texas & 21 & 27.3 & 353 & 34.3 & 15,257 & 580.9 & 14,302 & 177.8 & 7 & 39.1 & 11,538 & 113.5 & 1,736 & 723.5 & 43,215 & 194.7 \\
\hline Utah & 13 & 57.0 & 26 & 44.3 & 118 & 503.5 & 342 & 118.0 & 3 & 14.2 & 921 & 49.2 & 28 & 80.9 & 1,451 & 62.5 \\
\hline Vermont & 0 & 0.0 & 3 & 34.4 & 32 & 551.4 & 33 & 362.7 & 1 & 609.8 & 266 & 52.2 & 14 & 188.7 & 349 & 64.3 \\
\hline Virginia & 8 & 41.8 & 124 & 28.0 & 5,798 & 437.1 & 976 & 173.4 & 4 & 80.3 & 2,913 & 64.2 & 346 & 267.5 & 10,170 & 144.8 \\
\hline Washington & 80 & 104.9 & 213 & 42.6 & 1,021 & 469.2 & 976 & 156.2 & 31 & 82.3 & 4,074 & 93.6 & 448 & 242.9 & 6,848 & 114.3 \\
\hline West Virginia & 0 & 0.0 & 2 & 15.6 & 208 & 383.0 & 43 & 207.8 & 0 & 0.0 & 686 & 46.9 & 63 & 359.0 & 1,002 & 63.8 \\
\hline Wisconsin & 14 & 34.6 & 30 & 24.6 & 1,037 & 367.8 & 454 & 169.3 & 0 & 0.0 & 1,344 & 32.9 & 94 & 176.1 & 2,973 & 61.3 \\
\hline Wyoming & 8 & 85.8 & 1 & 22.2 & 9 & 156.5 & 33 & 77.7 & 0 & 0.0 & 100 & 24.1 & 4 & 64.3 & 155 & 32.0 \\
\hline Subtotal & 1,492 & 77.7 & 6,206 & 42.4 & 214,845 & 657.9 & 120,725 & 281.0 & 451 & 100.1 & 155,931 & 91.0 & 22,312 & 525.2 & 522,007 & 194.7 \\
\hline
\end{tabular}

\section{U.S. dependent areas ${ }^{\mathrm{e}}$}



\begin{tabular}{|c|c|c|c|c|c|c|c|c|c|c|c|c|c|c|c|c|}
\hline Guam & 0 & - & 14 & - & 1 & - & 0 & - & 7 & - & 5 & - & 2 & - & 29 & 22.9 \\
\hline Northern Mariana Islands & 0 & - & 1 & - & 0 & - & 0 & - & 2 & - & 0 & - & 0 & - & 3 & 7.5 \\
\hline Puerto Rico & 1 & - & 0 & - & 1 & - & 9,202 & - & 0 & - & 7 & - & 0 & - & 9,211 & 309.5 \\
\hline Republic of Palau & 0 & - & 1 & - & 0 & - & 0 & - & 3 & - & 0 & - & 0 & - & 4 & 22.6 \\
\hline U.S. Virgin Islands & 0 & - & 1 & - & 169 & - & 112 & - & 0 & - & 26 & - & 1 & - & 309 & 351.9 \\
\hline Subtotal & 1 & - & 17 & - & 171 & - & 9,314 & - & 13 & - & 38 & - & 3 & - & 9,557 & 290.4 \\
\hline Total & 1,493 & - & 6,223 & - & 215,016 & - & 130,039 & - & 464 & - & 155,969 & - & 22,315 & - & 531,564 & 195.8 \\
\hline
\end{tabular}

Note. Data are based on address of residence at the end of the specified year (i.e., most recent known address). Numbers less than 12, and rates based on these numbers, should be interpreted with caution.

${ }^{a}$ Includes Asian/Pacific Islander legacy cases (see Technical Notes).

${ }^{b}$ Hispanics/Latinos can be of any race.

${ }^{c}$ Includes persons whose race/ethnicity is unknown.

d Rates are per 100,000 population.

으

e Rates by race/ethnicity are not provided because U.S. census information for U.S. dependent areas is limited. 
Table 28. Diagnoses of HIV infection, 2016, and persons living with diagnosed HIV infection (prevalence), yearend 2015, by metropolitan statistical area of residence-United States and Puerto Rico

\begin{tabular}{|c|c|c|c|c|c|}
\hline \multirow[b]{2}{*}{ MSA of residence } & \multicolumn{3}{|c|}{ Diagnosis, $2016^{a}$} & \multicolumn{2}{|c|}{$\begin{array}{l}\text { Prevalence of diagnosed HIV } \\
\text { infection, year-end } 2015^{\mathrm{b}}\end{array}$} \\
\hline & No. & Rate $^{\text {C }}$ & Rank $^{\mathrm{d}}$ & No. & Rate $^{\mathrm{C}}$ \\
\hline Akron, $\mathrm{OH}$ & 62 & 8.8 & 66 & 896 & 127.4 \\
\hline Albany-Schenectady-Troy, NY & 58 & 6.6 & 83 & 1,864 & 211.6 \\
\hline Albuquerque, NM & 57 & 6.3 & 85 & 1,614 & 178.3 \\
\hline Allentown-Bethlehem-Easton, PA-NJ & 50 & 6.0 & 89 & 1,450 & 174.1 \\
\hline Atlanta-Sandy Springs-Roswell, GA & 1,701 & 29.4 & 4 & 30,884 & 541.9 \\
\hline Augusta-Richmond County, GA-SC & 94 & 15.8 & 27 & 2,197 & 372.3 \\
\hline Austin-Round Rock, TX & 308 & 15.0 & 30 & 5,625 & 281.5 \\
\hline Bakersfield, CA & 112 & 12.7 & 41 & 1,531 & 174.1 \\
\hline Baltimore-Columbia-Towson, MD & 531 & 19.0 & 14 & 17,896 & 640.6 \\
\hline Baton Rouge, LA & 252 & 30.2 & 3 & 4,990 & 601.4 \\
\hline Birmingham-Hoover, AL & 112 & 9.8 & 62 & 3,950 & 345.0 \\
\hline Boise City, ID & 17 & 2.5 & 106 & 325 & 48.1 \\
\hline Boston-Cambridge-Newton, MA-NH ${ }^{e}$ & 510 & 10.6 & 55 & 13,660 & 286.6 \\
\hline Boston Division & 269 & 13.5 & - & 7,917 & 399.6 \\
\hline Cambridge Division & 233 & 9.8 & - & 5,425 & 230.2 \\
\hline Bridgeport-Stamford-Norwalk, CT & 73 & 7.7 & 73 & 2,590 & 274.2 \\
\hline Buffalo-Cheektowaga-Niagara Falls, NY & 92 & 8.1 & 69 & 2,393 & 210.7 \\
\hline Cape Coral-Fort Myers, FL & 103 & 14.3 & 36 & 2,039 & 291.2 \\
\hline Charleston-North Charleston, SC & 116 & 15.2 & 29 & 2,496 & 335.2 \\
\hline Charlotte-Concord-Gastonia, NC-SC & 404 & 16.3 & 24 & 7,750 & 319.6 \\
\hline Chattanooga, TN-GA & 59 & 10.7 & 54 & 1,059 & 193.6 \\
\hline Chicago-Naperville-Elgin, IL-IN-WI & 1,263 & 13.3 & 37 & 29,459 & 309.0 \\
\hline Chicago Division & 1,125 & 15.4 & - & 26,962 & 368.1 \\
\hline Elgin Division & 36 & 5.7 & - & 383 & 60.4 \\
\hline Gary Division & 60 & 8.6 & - & 1,155 & 164.3 \\
\hline Lake County Division & 42 & 4.8 & - & 959 & 110.0 \\
\hline Cincinnati, OH-KY-IN & 209 & 9.7 & 63 & 3,793 & 176.0 \\
\hline Cleveland-Elyria, $\mathrm{OH}$ & 228 & 11.1 & 47 & 4,992 & 242.3 \\
\hline Colorado Springs, CO & 40 & 5.6 & 95 & 805 & 115.4 \\
\hline Columbia, SC & 177 & 21.7 & 11 & 3,854 & 475.9 \\
\hline Columbus, $\mathrm{OH}$ & 236 & 11.6 & 46 & 5,301 & 262.4 \\
\hline Dallas-Fort Worth-Arlington, TX & 1,348 & 18.6 & 15 & 25,024 & 353.0 \\
\hline Dallas Division & 1,025 & 21.4 & - & 19,650 & 418.5 \\
\hline Fort Worth Division & 323 & 13.2 & - & 5,374 & 224.5 \\
\hline Dayton, $\mathrm{OH}$ & 64 & 8.0 & 70 & 1,490 & 186.3 \\
\hline Deltona-Daytona Beach-Ormond Beach, FL & 94 & 14.7 & 32 & 1,774 & 284.6 \\
\hline Denver-Aurora-Lakewood, CO & 303 & 10.6 & 56 & 8,831 & 314.4 \\
\hline Des Moines-West Des Moines, IA & 38 & 6.0 & 88 & 776 & 124.6 \\
\hline Detroit-Warren-Dearborn, MI & 509 & 11.8 & 43 & 9,574 & 222.8 \\
\hline Detroit Division & 341 & 19.5 & - & 6,606 & 376.0 \\
\hline Warren Division & 168 & 6.6 & - & 2,968 & 116.8 \\
\hline Durham-Chapel Hill, NC & 103 & 18.4 & 18 & 2,154 & 390.8 \\
\hline El Paso, TX & 101 & 12.0 & 42 & 2,026 & 242.0 \\
\hline Fayetteville-Springdale-Rogers, AR-MO & 29 & 5.5 & 96 & 598 & 116.5 \\
\hline Fresno, CA & 128 & 13.1 & 39 & 1,659 & 170.7 \\
\hline Grand Rapids-Wyoming, MI & 49 & 4.7 & 101 & 1,100 & 105.9 \\
\hline Greensboro-High Point, NC & 159 & 21.0 & 12 & 2,540 & 337.9 \\
\hline Greenville-Anderson-Mauldin, SC & 95 & 10.7 & 52 & 1,871 & 214.3 \\
\hline Harrisburg-Carlisle, PA & 61 & 10.7 & 51 & 1,207 & 213.6 \\
\hline Hartford-West Hartford-East Hartford, CT & 72 & 6.0 & 90 & 3,213 & 265.5 \\
\hline Honolulu (Urban), $\mathrm{HI}$ & 62 & 6.2 & 86 & 1,736 & 174.8 \\
\hline
\end{tabular}


Table 28. Diagnoses of HIV infection, 2016, and persons living with diagnosed HIV infection (prevalence), yearend 2015, by metropolitan statistical area of residence-United States and Puerto Rico (cont)

\begin{tabular}{|c|c|c|c|c|c|}
\hline \multirow[b]{2}{*}{ MSA of residence } & \multicolumn{3}{|c|}{ Diagnosis, $2016^{a}$} & \multicolumn{2}{|c|}{$\begin{array}{l}\text { Prevalence of diagnosed HIV } \\
\text { infection, year-end } 2015^{\mathrm{b}}\end{array}$} \\
\hline & No. & Rate $^{\mathrm{c}}$ & $\operatorname{Rank}^{\mathrm{d}}$ & No. & Rate $^{\mathrm{C}}$ \\
\hline Houston-The Woodlands-Sugar Land, TX & 1,475 & 21.8 & 10 & 27,639 & 415.8 \\
\hline Indianapolis-Carmel-Anderson, IN & 236 & 11.8 & 44 & 5,293 & 266.4 \\
\hline Jackson, MS & 145 & 25.0 & 6 & 3,322 & 574.1 \\
\hline Jacksonville, FL & 339 & 22.9 & 8 & 6,629 & 457.8 \\
\hline Kansas City, MO-KS & 216 & 10.3 & 59 & 4,447 & 213.3 \\
\hline Knoxville, TN & 64 & 7.4 & 78 & 1,084 & 125.9 \\
\hline Lakeland-Winter Haven, FL & 124 & 18.6 & 16 & 2,165 & 333.4 \\
\hline Lancaster, PA & 39 & 7.2 & 79 & 715 & 133.3 \\
\hline Las Vegas-Henderson-Paradise, NV & 470 & 21.8 & 9 & 7,436 & 352.5 \\
\hline Lexington-Fayette, $\mathrm{KY}$ & 53 & 10.5 & 57 & 1,055 & 210.7 \\
\hline Little Rock-North Little Rock-Conway, AR & 144 & 19.6 & 13 & 2,032 & 277.8 \\
\hline Los Angeles-Long Beach-Anaheim, CA & 2,198 & 16.5 & 23 & 54,386 & 409.9 \\
\hline Anaheim Division & 353 & 11.1 & - & 6,185 & 195.9 \\
\hline Los Angeles Division & 1,845 & 18.2 & - & 48,201 & 476.7 \\
\hline Louisville/Jefferson County, KY-IN & 187 & 14.6 & 34 & 2,437 & 190.8 \\
\hline Madison, WI & 26 & 4.0 & 103 & 702 & 109.6 \\
\hline McAllen-Edinburg-Mission, TX & 83 & 9.8 & 61 & 1,131 & 134.8 \\
\hline Memphis, TN-MS-AR & 308 & 22.9 & 7 & 6,600 & 491.8 \\
\hline Miami-Fort Lauderdale-West Palm Beach, FL & 2,346 & 38.7 & 1 & 52,727 & 878.5 \\
\hline Fort Lauderdale Division & 766 & 40.1 & - & 18,890 & $1,000.9$ \\
\hline Miami Division & 1,275 & 47.0 & - & 25,984 & 965.0 \\
\hline West Palm Beach Division & 305 & 21.1 & - & 7,853 & 552.3 \\
\hline Milwaukee-Waukesha-West Allis, WI & 121 & 7.7 & 75 & 3,040 & 193.1 \\
\hline Minneapolis-St. Paul-Bloomington, MN-WI & 248 & 7.0 & 80 & 6,527 & 185.5 \\
\hline Modesto, CA & 21 & 3.9 & 104 & 561 & 104.8 \\
\hline Nashville-Davidson-Murfreesboro-Franklin, TN & 194 & 10.4 & 58 & 4,769 & 260.7 \\
\hline New Haven-Milford, CT & 81 & 9.5 & 64 & 3,173 & 369.4 \\
\hline New Orleans-Metairie, LA & 422 & 33.3 & 2 & 7,459 & 591.0 \\
\hline New York-Newark-Jersey City, NY-NJ-PA & 3,397 & 16.9 & 22 & 138,360 & 687.7 \\
\hline Nassau County Division & 216 & 7.6 & - & 5,913 & 206.9 \\
\hline New York Division & 2,707 & 18.8 & - & 117,506 & 818.2 \\
\hline Newark Division & 451 & 18.0 & - & 13,826 & 552.1 \\
\hline North Port-Sarasota-Bradenton, FL & 61 & 7.7 & 72 & 1,920 & 250.0 \\
\hline Ogden-Clearfield, UT & 11 & 1.7 & 108 & 351 & 54.6 \\
\hline Oklahoma City, OK & 149 & 10.9 & 50 & 2,628 & 193.7 \\
\hline Omaha-Council Bluffs, NE-IA & 53 & 5.7 & 93 & 590 & 64.5 \\
\hline Orlando-Kissimmee-Sanford, FL & 668 & 27.4 & 5 & 10,984 & 461.1 \\
\hline Oxnard-Thousand Oaks-Ventura, CA & 45 & 5.3 & 97 & 923 & 108.9 \\
\hline Palm Bay-Melbourne-Titusville, FL & 64 & 11.1 & 48 & 1,451 & 255.5 \\
\hline Philadelphia-Camden-Wilmington, PA-NJ-DE-MD & 790 & 13.0 & 40 & 24,857 & 410.0 \\
\hline Camden Division & 103 & 8.2 & - & 2,977 & 237.8 \\
\hline Montgomery County Division & 69 & 3.5 & - & 1,524 & 77.7 \\
\hline Philadelphia Division & 536 & 25.1 & - & 17,969 & 844.3 \\
\hline Wilmington Division & 82 & 11.3 & - & 2,387 & 330.8 \\
\hline Phoenix-Mesa-Scottsdale, AZ & 612 & 13.1 & 38 & 11,370 & 248.9 \\
\hline Pittsburgh, PA & 146 & 6.2 & 87 & 3,238 & 137.7 \\
\hline Portland-South Portland, ME & 19 & 3.6 & 105 & 800 & 151.9 \\
\hline Portland-Vancouver-Hillsboro, OR-WA & 164 & 6.8 & 82 & 5,235 & 219.5 \\
\hline Providence-Warwick, RI-MA & 106 & 6.6 & 84 & 3,537 & 219.3 \\
\hline Provo-Orem, UT & 13 & 2.2 & 107 & 186 & 31.8 \\
\hline Raleigh, NC & 191 & 14.7 & 33 & 3,649 & 287.0 \\
\hline
\end{tabular}


Table 28. Diagnoses of HIV infection, 2016, and persons living with diagnosed HIV infection (prevalence), yearend 2015, by metropolitan statistical area of residence-United States and Puerto Rico (cont)

\begin{tabular}{|c|c|c|c|c|c|}
\hline \multirow[b]{2}{*}{ MSA of residence } & \multicolumn{3}{|c|}{ Diagnosis, $2016^{a}$} & \multicolumn{2}{|c|}{$\begin{array}{l}\text { Prevalence of diagnosed HIV } \\
\text { infection, year-end } 2015^{\mathrm{b}}\end{array}$} \\
\hline & No. & Rate $^{c}$ & Rank $^{d}$ & No. & Rate $^{\mathrm{C}}$ \\
\hline Richmond, VA & 184 & 14.4 & 35 & 4,431 & 348.8 \\
\hline Riverside-San Bernardino-Ontario, CA & 486 & 10.7 & 53 & 7,662 & 171.2 \\
\hline Rochester, NY & 61 & 5.7 & 94 & 2,902 & 268.4 \\
\hline Sacramento-Roseville-Arden-Arcade, CA & 229 & 10.0 & 60 & 4,081 & 180.0 \\
\hline St. Louis, MO-IL & 308 & 11.0 & 49 & 6,815 & 242.7 \\
\hline Salt Lake City, UT & 101 & 8.5 & 67 & 1,894 & 162.2 \\
\hline San Antonio-New Braunfels, TX & 390 & 16.1 & 25 & 6,216 & 261.0 \\
\hline San Diego-Carlsbad, CA & 494 & 14.9 & 31 & 11,336 & 344.5 \\
\hline San Francisco-Oakland-Hayward, $\mathrm{CA}^{\mathrm{e}}$ & 741 & 15.8 & 26 & 23,216 & 500.1 \\
\hline Oakland Division & 398 & 14.3 & - & 7,429 & 269.6 \\
\hline San Francisco Division & 315 & 19.3 & - & 14,983 & 921.9 \\
\hline San Jose-Sunnyvale-Santa Clara, CA & 137 & 6.9 & 81 & 2,986 & 151.7 \\
\hline San Juan-Carolina-Caguas, PR & 397 & 18.4 & 19 & 9,235 & 420.6 \\
\hline Santa Rosa, CA & 24 & 4.8 & 100 & 1,210 & 241.3 \\
\hline Scranton-Wilkes-Barre-Hazelton, PA & 28 & 5.0 & 99 & 628 & 112.6 \\
\hline Seattle-Tacoma-Bellevue, WA & 315 & 8.3 & 68 & 8,762 & 235.1 \\
\hline Seattle Division & 266 & 9.1 & - & 7,431 & 257.6 \\
\hline Tacoma Division & 49 & 5.7 & - & 1,331 & 157.9 \\
\hline Spokane-Spokane Valley, WA & 24 & 4.3 & 102 & 579 & 105.8 \\
\hline Springfield, MA & 73 & 11.6 & 45 & 2,085 & 330.9 \\
\hline Stockton-Lodi, CA & 55 & 7.5 & 76 & 1,296 & 179.1 \\
\hline Syracuse, NY & 38 & 5.8 & 91 & 1,230 & 186.4 \\
\hline Tampa-St. Petersburg-Clearwater, FL & 560 & 18.5 & 17 & 11,937 & 401.8 \\
\hline Toledo, $\mathrm{OH}$ & 47 & 7.8 & 71 & 971 & 160.3 \\
\hline Tucson, AZ & 90 & 8.9 & 65 & 2,368 & 234.9 \\
\hline Tulsa, OK & 76 & 7.7 & 74 & 1,802 & 183.8 \\
\hline Virginia Beach-Norfolk-Newport News, VA-NC & 297 & 17.2 & 21 & 5,473 & 317.6 \\
\hline Washington-Arlington-Alexandria, DC-VA-MD-WV & 1,122 & 18.3 & 20 & 21,522 & 354.1 \\
\hline Silver Spring Division & 195 & 15.1 & - & 4,067 & 317.3 \\
\hline Washington Division & 927 & 19.2 & - & 17,455 & 363.9 \\
\hline Wichita, KS & 37 & 5.7 & 92 & 866 & 134.7 \\
\hline Winston-Salem, NC & 101 & 15.3 & 28 & 1,791 & 272.3 \\
\hline Worcester, MA-CT & 70 & 7.5 & 77 & 1,944 & 208.1 \\
\hline Youngstown-Warren-Boardman, OH-PA & 28 & 5.1 & 98 & 767 & 139.6 \\
\hline Subtotal for MSAs (population of $\geq 500,000$ ) & 32,621 & 14.7 & - & 780,399 & 354.6 \\
\hline Metropolitan areas (population of $50,000-499,999$ ) & 4,891 & 8.4 & - & 98,716 & 170.1 \\
\hline Nonmetropolitan areas & 2,344 & 5.1 & - & 54,278 & 117.4 \\
\hline Total $^{f}$ & 40,305 & 12.3 & - & 990,637 & 305.4 \\
\hline
\end{tabular}

Abbreviation: MSA, metropolitan statistical area.

Note. Because of the lack of U.S. census information for all U.S. dependent areas, table includes data for only the United States and Puerto Rico. Data for the year 2016 are preliminary (subject to change) because they are based on only a 6-month reporting delay. Numbers less than 12, and rates based on these numbers, should be interpreted with caution.

MSA definitions for this report can be found at http://www.census.gov/programs-surveys/metro-micro.html.

a Data are based on residence at time of diagnosis of HIV infection.

${ }^{b}$ Data are based on address of residence at the end of the specified year (i.e., most recent known address).

${ }^{\mathrm{C}}$ Rates are per 100,000 population.

d Based on rate.

e Counts of diagnoses of HIV infection for the metropolitan divisions do not sum to the MSA total. MSA total includes data from 1 metropolitan division with population of $<500,000$.

$\mathrm{f}$ Includes persons whose county of residence is unknown. 


\begin{tabular}{|c|c|c|c|c|c|c|c|c|}
\hline \multirow[b]{3}{*}{ MSA of residence } & & & & \multicolumn{3}{|c|}{ Diagnosis, cumulative $^{a, b}$} & & \\
\hline & \multicolumn{3}{|c|}{ Diagnosis, $2016^{\mathrm{b}}$} & \multirow{2}{*}{$\begin{array}{c}\text { Adults or } \\
\text { adolescents } \\
\text { No. }\end{array}$} & \multirow{2}{*}{$\begin{array}{c}\text { Children } \\
\text { No. }\end{array}$} & \multirow{2}{*}{$\begin{array}{l}\text { Total } \\
\text { No. }\end{array}$} & \multicolumn{2}{|c|}{$\begin{array}{l}\text { Prevalence of stage } 3 \\
\text { (AIDS), year-end } 2015^{C}\end{array}$} \\
\hline & No. & Rate $^{d}$ & Rank $^{\mathrm{e}}$ & & & & No. & Rate $^{d}$ \\
\hline Akron, $\mathrm{OH}$ & 21 & 3.0 & 84 & 964 & 1 & 965 & 399 & 56.7 \\
\hline Albany-Schenectady-Troy, NY & 22 & 2.5 & 90 & 2,679 & 28 & 2,707 & 1,073 & 121.8 \\
\hline Albuquerque, NM & 28 & 3.1 & 81 & 1,855 & 4 & 1,859 & 945 & 104.4 \\
\hline Allentown-Bethlehem-Easton, PA-NJ & 19 & 2.3 & 95 & 1,777 & 19 & 1,796 & 823 & 98.8 \\
\hline Atlanta-Sandy Springs-Roswell, GA & 695 & 12.0 & 7 & 34,229 & 151 & 34,380 & 16,414 & 288.0 \\
\hline Augusta-Richmond County, GA-SC & 48 & 8.1 & 19 & 2,682 & 23 & 2,705 & 1,101 & 186.6 \\
\hline Austin-Round Rock, TX & 101 & 4.9 & 49 & 6,376 & 26 & 6,402 & 3,032 & 151.7 \\
\hline Bakersfield, CA & 29 & 3.3 & 79 & 2,129 & 9 & 2,138 & 854 & 97.1 \\
\hline Baltimore-Columbia-Towson, MD & 315 & 11.3 & 8 & 26,080 & 229 & 26,309 & 9,922 & 355.1 \\
\hline Baton Rouge, LA & 150 & 18.0 & 1 & 5,795 & 24 & 5,819 & 2,641 & 318.3 \\
\hline Birmingham-Hoover, AL & 61 & 5.3 & 42 & 3,567 & 25 & 3,592 & 1,684 & 147.1 \\
\hline Boise City, ID & 13 & 1.9 & 105 & 440 & 0 & 440 & 115 & 17.0 \\
\hline Boston-Cambridge-Newton, MA-NH ${ }^{f}$ & 174 & 3.6 & 69 & 17,128 & 162 & 17,290 & 7,656 & 160.6 \\
\hline Boston Division & 90 & 4.5 & - & 10,579 & 97 & 10,676 & 4,500 & 227.1 \\
\hline Cambridge Division & 79 & 3.3 & - & 6,162 & 64 & 6,226 & 2,999 & 127.2 \\
\hline Bridgeport-Stamford-Norwalk, CT & 34 & 3.6 & 72 & 4,314 & 57 & 4,371 & 1,554 & 164.5 \\
\hline Buffalo-Cheektowaga-Niagara Falls, NY & 40 & 3.5 & 74 & 3,089 & 20 & 3,109 & 1,265 & 111.4 \\
\hline Cape Coral-Fort Myers, FL & 57 & 7.9 & 20 & 2,307 & 25 & 2,332 & 1,177 & 168.1 \\
\hline Charleston-North Charleston, SC & 51 & 6.7 & 26 & 2,795 & 25 & 2,820 & 1,315 & 176.6 \\
\hline Charlotte-Concord-Gastonia, NC-SC & 175 & 7.1 & 23 & 6,164 & 30 & 6,194 & 3,402 & 140.3 \\
\hline Chattanooga, TN-GA & 20 & 3.6 & 70 & 1,338 & 3 & 1,341 & 563 & 102.9 \\
\hline Chicago-Naperville-Elgin, IL-IN-WI & 534 & 5.6 & 38 & 38,962 & 263 & 39,225 & 15,014 & 157.5 \\
\hline Chicago Division & 482 & 6.6 & - & 35,663 & 241 & 35,904 & 13,705 & 187.1 \\
\hline Elgin Division & 14 & 2.2 & - & 813 & 8 & 821 & 206 & 32.5 \\
\hline Gary Division & 23 & 3.3 & - & 1,441 & 9 & 1,450 & 568 & 80.8 \\
\hline Lake County Division & 15 & 1.7 & - & 1,045 & 5 & 1,050 & 535 & 61.4 \\
\hline Cincinnati, OH-KY-IN & 89 & 4.1 & 65 & 3,928 & 21 & 3,949 & 1,888 & 87.6 \\
\hline Cleveland-Elyria, $\mathrm{OH}$ & 120 & 5.8 & 34 & 5,387 & 49 & 5,436 & 2,359 & 114.5 \\
\hline Colorado Springs, CO & 17 & 2.4 & 94 & 758 & 5 & 763 & 369 & 52.9 \\
\hline Columbia, SC & 103 & 12.6 & 6 & 4,635 & 26 & 4,661 & 2,112 & 260.8 \\
\hline Columbus, $\mathrm{OH}$ & 131 & 6.4 & 28 & 4,879 & 24 & 4,903 & 2,386 & 118.1 \\
\hline
\end{tabular}




\begin{tabular}{|c|c|c|c|c|c|c|c|c|}
\hline \multirow[b]{3}{*}{ MSA of residence } & & & & \multicolumn{3}{|c|}{ Diagnosis, cumulative $\mathrm{e}^{\mathrm{a}, \mathrm{b}}$} & & \\
\hline & \multicolumn{3}{|c|}{ Diagnosis, $2016^{\mathrm{b}}$} & \multirow{2}{*}{$\begin{array}{c}\begin{array}{c}\text { Adults or } \\
\text { adolescents }\end{array} \\
\text { No. }\end{array}$} & \multirow{2}{*}{$\begin{array}{c}\text { Children } \\
\text { No. }\end{array}$} & \multirow{2}{*}{$\begin{array}{l}\text { Total } \\
\text { No. }\end{array}$} & \multicolumn{2}{|c|}{$\begin{array}{l}\text { Prevalence of stage } 3 \\
\text { (AIDS), year-end 2015 }\end{array}$} \\
\hline & No. & Rate $^{d}$ & Rank $^{\mathrm{e}}$ & & & & No. & Rate $^{d}$ \\
\hline Dallas-Fort Worth-Arlington, TX & 656 & 9.1 & 15 & 27,764 & 66 & 27,830 & 12,975 & 183.0 \\
\hline Dallas Division & 525 & 11.0 & - & 21,925 & 39 & 21,964 & 10,154 & 216.2 \\
\hline Fort Worth Division & 131 & 5.4 & - & 5,839 & 27 & 5,866 & 2,821 & 117.8 \\
\hline Dayton, $\mathrm{OH}$ & 32 & 4.0 & 66 & 1,647 & 14 & 1,661 & 758 & 94.8 \\
\hline Deltona-Daytona Beach-Ormond Beach, FL & 53 & 8.3 & 17 & 2,131 & 19 & 2,150 & 1,047 & 168.0 \\
\hline Denver-Aurora-Lakewood, CO & 124 & 4.3 & 60 & 8,702 & 24 & 8,726 & 3,805 & 135.5 \\
\hline Des Moines-West Des Moines, IA & 23 & 3.6 & 71 & 780 & 4 & 784 & 421 & 67.6 \\
\hline Detroit-Warren-Dearborn, MI & 273 & 6.4 & 29 & 13,711 & 79 & 13,790 & 5,060 & 117.7 \\
\hline Detroit Division & 179 & 10.2 & - & 10,708 & 62 & 10,770 & 3,594 & 204.5 \\
\hline Warren Division & 94 & 3.7 & - & 3,003 & 17 & 3,020 & 1,466 & 57.7 \\
\hline Durham-Chapel Hill, NC & 38 & 6.8 & 24 & 1,685 & 10 & 1,695 & 890 & 161.5 \\
\hline El Paso, TX & 44 & 5.2 & 43 & 2,162 & 10 & 2,172 & 1,142 & 136.4 \\
\hline Fayetteville-Springdale-Rogers, AR-MO & 13 & 2.5 & 93 & 530 & 6 & 536 & 309 & 60.2 \\
\hline Fresno, CA & 55 & 5.6 & 39 & 2,203 & 11 & 2,214 & 959 & 98.6 \\
\hline Grand Rapids-Wyoming, MI & 20 & 1.9 & 103 & 1,231 & 6 & 1,237 & 573 & 55.2 \\
\hline Greensboro-High Point, NC & 33 & 4.4 & 59 & 1,831 & 19 & 1,850 & 949 & 126.2 \\
\hline Greenville-Anderson-Mauldin, SC & 43 & 4.9 & 51 & 2,090 & 6 & 2,096 & 1,030 & 118.0 \\
\hline Harrisburg-Carlisle, PA & 33 & 5.8 & 35 & 1,661 & 9 & 1,670 & 648 & 114.7 \\
\hline Hartford-West Hartford-East Hartford, CT & 52 & 4.3 & 62 & 5,927 & 46 & 5,973 & 2,014 & 166.5 \\
\hline Honolulu (Urban), $\mathrm{HI}$ & 22 & 2.2 & 96 & 2,431 & 13 & 2,444 & 981 & 98.8 \\
\hline Houston-The Woodlands-Sugar Land, TX & 719 & 10.6 & 11 & 35,053 & 183 & 35,236 & 14,713 & 221.3 \\
\hline Indianapolis-Carmel-Anderson, IN & 107 & 5.3 & 41 & 5,470 & 26 & 5,496 & 2,676 & 134.7 \\
\hline Jackson, MS & 99 & 17.1 & 2 & 3,641 & 31 & 3,672 & 1,632 & 282.0 \\
\hline Jacksonville, FL & 194 & 13.1 & 5 & 8,424 & 79 & 8,503 & 3,755 & 259.3 \\
\hline Kansas City, MO-KS & 94 & 4.5 & 56 & 6,050 & 17 & 6,067 & 2,504 & 120.1 \\
\hline Knoxville, TN & 22 & 2.5 & 89 & 1,345 & 8 & 1,353 & 566 & 65.7 \\
\hline Lakeland-Winter Haven, FL & 68 & 10.2 & 12 & 2,593 & 22 & 2,615 & 1,288 & 198.3 \\
\hline Lancaster, PA & 20 & 3.7 & 68 & 913 & 22 & 935 & 353 & 65.8 \\
\hline Las Vegas-Henderson-Paradise, NV & 217 & 10.1 & 13 & 6,710 & 28 & 6,738 & 3,636 & 172.4 \\
\hline Lexington-Fayette, KY & 32 & 6.3 & 31 & 1,033 & 1 & 1,034 & 534 & 106.7 \\
\hline Little Rock-North Little Rock-Conway, AR & 55 & 7.5 & 22 & 1,941 & 15 & 1,956 & 866 & 118.4 \\
\hline
\end{tabular}


Table 29. Stage 3 (AIDS), 2016 and cumulative, and persons living with diagnosed HIV infection ever classified as stage 3 (AIDS) (prevalence), year-end 2015, by metropolitan statistical area of residence-United States and Puerto Rico (cont)

\begin{tabular}{|c|c|c|c|c|c|c|c|c|}
\hline \multirow[b]{3}{*}{ MSA of residence } & & & & \multicolumn{3}{|c|}{ Diagnosis, cumulative ${ }^{a, b}$} & & \\
\hline & \multicolumn{3}{|c|}{ Diagnosis, $2016^{\mathrm{b}}$} & \multirow{2}{*}{$\begin{array}{c}\text { Adults or } \\
\text { adolescents } \\
\text { No. }\end{array}$} & \multirow{2}{*}{$\begin{array}{c}\text { Children } \\
\text { No. }\end{array}$} & \multirow{2}{*}{$\begin{array}{l}\text { Total } \\
\text { No. }\end{array}$} & \multicolumn{2}{|c|}{$\begin{array}{l}\text { Prevalence of stage } 3 \\
\text { (AIDS), year-end } 2015^{\circ}\end{array}$} \\
\hline & No. & Rate $^{d}$ & Rank $^{\mathrm{e}}$ & & & & No. & Rate $^{d}$ \\
\hline Los Angeles-Long Beach-Anaheim, CA & 828 & 6.2 & 33 & 72,381 & 309 & 72,690 & 29,798 & 224.6 \\
\hline Anaheim Division & 120 & 3.8 & - & 8,544 & 45 & 8,589 & 3,317 & 105.1 \\
\hline Los Angeles Division & 708 & 7.0 & - & 63,837 & 264 & 64,101 & 26,481 & 261.9 \\
\hline Louisville/Jefferson County, KY-IN & 63 & 4.9 & 50 & 3,300 & 30 & 3,330 & 1,028 & 80.5 \\
\hline Madison, WI & 13 & 2.0 & 101 & 685 & 5 & 690 & 340 & 53.1 \\
\hline McAllen-Edinburg-Mission, TX & 44 & 5.2 & 45 & 1,128 & 13 & 1,141 & 643 & 76.6 \\
\hline Memphis, TN-MS-AR & 143 & 10.6 & 10 & 7,461 & 23 & 7,484 & 3,189 & 237.6 \\
\hline Miami-Fort Lauderdale-West Palm Beach, FL & 1,029 & 17.0 & 3 & 70,082 & 1,009 & 71,091 & 28,000 & 466.5 \\
\hline Fort Lauderdale Division & 360 & 18.9 & - & 21,521 & 266 & 21,787 & 10,142 & 537.4 \\
\hline Miami Division & 512 & 18.9 & - & 36,617 & 514 & 37,131 & 13,157 & 488.6 \\
\hline West Palm Beach Division & 157 & 10.9 & - & 11,944 & 229 & 12,173 & 4,701 & 330.6 \\
\hline Milwaukee-Waukesha-West Allis, WI & 56 & 3.6 & 73 & 3,316 & 19 & 3,335 & 1,501 & 95.3 \\
\hline Minneapolis-St. Paul-Bloomington, MN-WI & 107 & 3.0 & 83 & 5,977 & 24 & 6,001 & 3,006 & 85.4 \\
\hline Modesto, CA & 19 & 3.5 & 75 & 898 & 6 & 904 & 357 & 66.7 \\
\hline Nashville-Davidson-Murfreesboro-Franklin, TN & 81 & 4.3 & 61 & 5,245 & 25 & 5,270 & 2,391 & 130.7 \\
\hline New Haven-Milford, CT & 39 & 4.6 & 55 & 5,177 & 74 & 5,251 & 2,063 & 240.1 \\
\hline New Orleans-Metairie, LA & 179 & 14.1 & 4 & 11,414 & 80 & 11,494 & 3,968 & 314.4 \\
\hline New York-Newark-Jersey City, NY-NJ-PA ${ }^{f}$ & 1,779 & 8.8 & 16 & 228,870 & 3,018 & 231,888 & 79,546 & 395.4 \\
\hline Nassau County Division & 105 & 3.7 & - & 9,523 & 117 & 9,640 & 3,476 & 121.6 \\
\hline New York Division & 1,476 & 10.3 & - & 192,849 & 2,519 & 195,368 & 68,207 & 474.9 \\
\hline Newark Division & 192 & 7.7 & - & 24,528 & 369 & 24,897 & 7,181 & 286.8 \\
\hline North Port-Sarasota-Bradenton, FL & 40 & 5.1 & 46 & 2,505 & 29 & 2,534 & 1,094 & 142.4 \\
\hline Ogden-Clearfield, UT & 3 & 0.5 & 107 & 334 & 4 & 338 & 190 & 29.6 \\
\hline Oklahoma City, OK & 69 & 5.0 & 48 & 3,074 & 4 & 3,078 & 1,260 & 92.9 \\
\hline Omaha-Council Bluffs, NE-IA & 18 & 1.9 & 102 & 1,440 & 5 & 1,445 & 296 & 32.4 \\
\hline Orlando-Kissimmee-Sanford, FL & 273 & 11.2 & 9 & 12,128 & 95 & 12,223 & 5,859 & 246.0 \\
\hline Oxnard-Thousand Oaks-Ventura, CA & 18 & 2.1 & 100 & 1,277 & 5 & 1,282 & 512 & 60.4 \\
\hline Palm Bay-Melbourne-Titusville, FL & 28 & 4.8 & 52 & 1,869 & 13 & 1,882 & 868 & 152.8 \\
\hline
\end{tabular}




\begin{tabular}{|c|c|c|c|c|c|c|c|c|}
\hline \multirow[b]{3}{*}{ MSA of residence } & & & & \multicolumn{3}{|c|}{ Diagnosis, cumulative ${ }^{a, b}$} & & \\
\hline & \multicolumn{3}{|c|}{ Diagnosis, $2016^{\mathrm{b}}$} & \multirow{2}{*}{$\begin{array}{c}\text { Adults or } \\
\text { adolescents } \\
\text { No. }\end{array}$} & \multirow{2}{*}{$\begin{array}{c}\text { Children } \\
\text { No. }\end{array}$} & \multirow{2}{*}{$\begin{array}{l}\text { Total } \\
\text { No. }\end{array}$} & \multicolumn{2}{|c|}{$\begin{array}{l}\text { Prevalence of stage } 3 \\
\text { (AIDS), year-end } 2015^{C}\end{array}$} \\
\hline & No. & Rate $^{d}$ & Rank $^{\mathrm{e}}$ & & & & No. & Rate $^{d}$ \\
\hline Philadelphia-Camden-Wilmington, PA-NJ-DE-MD & 381 & 6.3 & 32 & 35,436 & 319 & 35,755 & 13,728 & 226.4 \\
\hline Camden Division & 42 & 3.4 & - & 3,959 & 43 & 4,002 & 1,492 & 119.2 \\
\hline Montgomery County Division & 42 & 2.1 & - & 2,918 & 12 & 2,930 & 775 & 39.5 \\
\hline Philadelphia Division & 249 & 11.7 & - & 24,801 & 240 & 25,041 & 10,026 & 471.1 \\
\hline Wilmington Division & 48 & 6.6 & - & 3,758 & 24 & 3,782 & 1,435 & 198.9 \\
\hline Phoenix-Mesa-Scottsdale, AZ & 236 & 5.1 & 47 & 10,403 & 31 & 10,434 & 5,444 & 119.2 \\
\hline Pittsburgh, PA & 66 & 2.8 & 85 & 4,107 & 22 & 4,129 & 1,697 & 72.2 \\
\hline Portland-South Portland, ME & 10 & 1.9 & 104 & 749 & 1 & 750 & 403 & 76.5 \\
\hline Portland-Vancouver-Hillsboro, OR-WA & 67 & 2.8 & 86 & 6,090 & 9 & 6,099 & 2,942 & 123.4 \\
\hline Providence-Warwick, RI-MA & 40 & 2.5 & 92 & 4,836 & 45 & 4,881 & 2,085 & 129.3 \\
\hline Provo-Orem, UT & 1 & 0.2 & 108 & 160 & 3 & 163 & 94 & 16.1 \\
\hline Raleigh, NC & 75 & 5.8 & 36 & 3,144 & 19 & 3,163 & 1,720 & 135.3 \\
\hline Richmond, VA & 70 & 5.5 & 40 & 4,508 & 35 & 4,543 & 1,968 & 154.9 \\
\hline Riverside-San Bernardino-Ontario, CA & 212 & 4.7 & 53 & 11,244 & 63 & 11,307 & 4,327 & 96.7 \\
\hline Rochester, NY & 35 & 3.2 & 80 & 3,749 & 17 & 3,766 & 1,641 & 151.8 \\
\hline Sacramento-Roseville-Arden-Arcade, CA & 97 & 4.2 & 64 & 5,131 & 30 & 5,161 & 2,184 & 96.3 \\
\hline St. Louis, MO-IL & 131 & 4.7 & 54 & 7,866 & 45 & 7,911 & 3,443 & 122.6 \\
\hline Salt Lake City, UT & 32 & 2.7 & 88 & 2,071 & 9 & 2,080 & 999 & 85.6 \\
\hline San Antonio-New Braunfels, TX & 165 & 6.8 & 25 & 7,036 & 32 & 7,068 & 3,292 & 138.2 \\
\hline San Diego-Carlsbad, CA & 189 & 5.7 & 37 & 15,858 & 71 & 15,929 & 6,222 & 189.1 \\
\hline San Francisco-Oakland-Hayward, $\mathrm{CA}^{f}$ & 243 & 5.2 & 44 & 46,313 & 102 & 46,415 & 13,812 & 297.5 \\
\hline Oakland Division & 119 & 4.3 & - & 12,116 & 53 & 12,169 & 4,521 & 164.0 \\
\hline San Francisco Division & 114 & 7.0 & - & 32,477 & 44 & 32,521 & 8,786 & 540.6 \\
\hline San Jose-Sunnyvale-Santa Clara, CA & 68 & 3.4 & 77 & 4,869 & 17 & 4,886 & 1,880 & 95.5 \\
\hline San Juan-Carolina-Caguas, PR & 162 & 7.5 & 21 & 24,995 & 278 & 25,273 & 4,678 & 213.0 \\
\hline Santa Rosa, CA & 5 & 1.0 & 106 & 2,164 & 8 & 2,172 & 789 & 157.3 \\
\hline Scranton-Wilkes-Barre-Hazelton, PA & 15 & 2.7 & 87 & 743 & 7 & 750 & 327 & 58.6 \\
\hline Seattle-Tacoma-Bellevue, WA & 125 & 3.3 & 78 & 11,214 & 28 & 11,242 & 4,663 & 125.1 \\
\hline Seattle Division & 99 & 3.4 & - & 9,884 & 18 & 9,902 & 3,957 & 137.2 \\
\hline Tacoma Division & 26 & 3.0 & - & 1,330 & 10 & 1,340 & 706 & 83.8 \\
\hline Spokane-Spokane Valley, WA & 12 & 2.2 & 97 & 677 & 1 & 678 & 338 & 61.7 \\
\hline
\end{tabular}


Table 29. Stage 3 (AIDS), 2016 and cumulative, and persons living with diagnosed HIV infection ever classified as stage 3 (AIDS) (prevalence), year-end 2015, by metropolitan statistical area of residence-United States and Puerto Rico (cont)

\begin{tabular}{|c|c|c|c|c|c|c|c|c|}
\hline \multirow[b]{3}{*}{ MSA of residence } & & & & \multicolumn{3}{|c|}{ Diagnosis, cumulative ${ }^{a, b}$} & & \\
\hline & \multicolumn{3}{|c|}{ Diagnosis, $2016^{\mathrm{b}}$} & \multirow{2}{*}{$\begin{array}{c}\text { Adults or } \\
\text { adolescents } \\
\text { No. }\end{array}$} & \multirow{2}{*}{$\begin{array}{c}\text { Children } \\
\text { No. }\end{array}$} & \multirow{2}{*}{$\begin{array}{l}\text { Total } \\
\text { No. }\end{array}$} & \multicolumn{2}{|c|}{$\begin{array}{l}\text { Prevalence of stage } 3 \\
\text { (AIDS), year-end } 2015^{\circ}\end{array}$} \\
\hline & No. & Rate $^{d}$ & Rank $^{\mathrm{e}}$ & & & & No. & Rate $^{d}$ \\
\hline Springfield, MA & 28 & 4.4 & 58 & 2,576 & 28 & 2,604 & 1,194 & 189.5 \\
\hline Stockton-Lodi, CA & 29 & 4.0 & 67 & 1,509 & 15 & 1,524 & 712 & 98.4 \\
\hline Syracuse, NY & 28 & 4.3 & 63 & 1,632 & 10 & 1,642 & 706 & 107.0 \\
\hline Tampa-St. Petersburg-Clearwater, FL & 291 & 9.6 & 14 & 14,927 & 116 & 15,043 & 6,734 & 226.7 \\
\hline Toledo, $\mathrm{OH}$ & 13 & 2.1 & 98 & 1,052 & 15 & 1,067 & 462 & 76.3 \\
\hline Tucson, AZ & 31 & 3.1 & 82 & 2,538 & 12 & 2,550 & 1,233 & 122.3 \\
\hline Tulsa, OK & 44 & 4.5 & 57 & 2,037 & 11 & 2,048 & 920 & 93.8 \\
\hline Virginia Beach-Norfolk-Newport News, VA-NC & 114 & 6.6 & 27 & 6,310 & 66 & 6,376 & 2,199 & 127.6 \\
\hline Washington-Arlington-Alexandria, DC-VA-MD-WV & 506 & 8.3 & 18 & 40,298 & 336 & 40,634 & 11,034 & 181.5 \\
\hline Silver Spring Division & 83 & 6.4 & - & 4,091 & 28 & 4,119 & 2,084 & 162.6 \\
\hline Washington Division & 423 & 8.7 & - & 36,207 & 308 & 36,515 & 8,950 & 186.6 \\
\hline Wichita, KS & 16 & 2.5 & 91 & 1,060 & 2 & 1,062 & 451 & 70.1 \\
\hline Winston-Salem, NC & 42 & 6.3 & 30 & 1,414 & 11 & 1,425 & 751 & 114.2 \\
\hline Worcester, MA-CT & 20 & 2.1 & 99 & 2,555 & 22 & 2,577 & 1,221 & 130.7 \\
\hline Youngstown-Warren-Boardman, $\mathrm{OH}-\mathrm{PA}$ & 19 & 3.5 & 76 & 765 & 0 & 765 & 358 & 65.2 \\
\hline Subtotal for MSAs (population of $\geq 500,000$ ) & 14,635 & 6.6 & - & $1,064,513$ & 8,649 & $1,073,162$ & 418,707 & 190.2 \\
\hline Metropolitan areas (population of $50,000-499,999$ ) & 2,334 & 4.0 & - & 121,714 & 844 & 122,558 & 52,272 & 90.1 \\
\hline Nonmetropolitan areas & 1,258 & 2.7 & - & 66,018 & 439 & 66,457 & 29,381 & 63.6 \\
\hline Total $^{\mathrm{g}}$ & 18,396 & 5.6 & - & $1,257,692$ & 9,977 & $1,267,669$ & 531,495 & 163.9 \\
\hline
\end{tabular}

Abbreviation: MSA, metropolitan statistical area.


change) because they are based on only a 6-month reporting delay. Numbers less than 12, and rates based on these numbers, should be interpreted with caution.

MSA definitions for this report can be found at http://www.census.gov/programs-surveys/metro-micro.html.

a From the beginning of the epidemic through 2016.

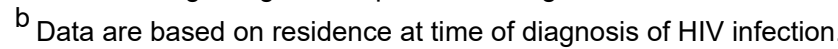

C Data are based on address of residence at the end of the specified year (i.e., most recent known address).

${ }^{d}$ Rates are per 100,000 population.

Based on rate.

${ }^{\mathrm{f}}$ Counts of stage 3 (AIDS) classifications for the metropolitan divisions do not sum to the MSA total. MSA total includes data from 1 metropolitan division with population of $<500,000$.

$\mathrm{g}$ Includes persons whose county of residence is unknown. 


\section{Web Addresses for Reports of State and Local HIV Surveillance}

\begin{tabular}{|c|c|}
\hline Alabama & http://www.adph.org/aids/Default.asp?id=984 \\
\hline Alaska & http://epibulletins.dhss.alaska.gov/Bulletin/DisplayClassificationBulletins/46 \\
\hline Arizona & http://www.azdhs.gov/phs/edc/odis/hiv-epidemiology/reports/index.php?pg=annual \\
\hline Arkansas & http://www.healthy.arkansas.gov/programs-services/topics/hiv-prevention \\
\hline California & http://www.cdph.ca.gov/Programs/CID/DOA/Pages/OAsre.aspx \\
\hline Chicago & http://www.cityofchicago.org/city/en/depts/cdph/provdrs/data_reports_and_administration/svcs/get_sti_hiv_datainchicago.html \\
\hline Colorado & https://www.colorado.gov/pacific/cdphe/stihiv-data-and-trends \\
\hline Connecticut & http://www.ct.gov/dph/cwp/view.asp?a=3135\&q=393048 \\
\hline Delaware & http://www.dhss.delaware.gov/dhss/dph/epi/disstatshiv.html \\
\hline District of Columbia & http://doh.dc.gov/service/hiv-reports-and-publications \\
\hline Florida & http://www.floridahealth.gov/diseases-and-conditions/aids/surveillance/index.html \\
\hline Georgia & http://dph.georgia.gov/georgias-hivaids-epidemiology-surveillance-section \\
\hline Guam & http://dphss.guam.gov/content/hiv-surveillance-program \\
\hline Hawaii & http://health.hawaii.gov/harmreduction/hiv-aids/data-and-statistics/ \\
\hline Houston & http://www.houstontx.gov/health/HIV-STD/ \\
\hline Idaho & http://healthandwelfare.idaho.gov/Health/FamilyPlanningSTDHIV/STDStatistics/tabid/393/Default.aspx \\
\hline Illinois & http://www.idph.state.il.us/aids/stats.htm \\
\hline Indiana & http://www.in.gov/isdh/23266.htm \\
\hline lowa & http://idph.iowa.gov/hivstdhep/hiv/data \\
\hline Kansas & http://www.kdheks.gov/sti_hiv/epi_profiles.htm \\
\hline Kentucky & http://chfs.ky.gov/dph/epi/hivaids/surveillance.htm \\
\hline Los Angeles & http://publichealth.lacounty.gov/dhsp/Reports.htm \\
\hline Louisiana & http://new.dhh.louisiana.gov/index.cfm/newsroom/category/63 \\
\hline Maine & http://www.maine.gov/dhhs/mecdc/infectious-disease/hiv-std/data/hiv.shtml \\
\hline Maryland & http://phpa.health.maryland.gov/OIDEOR/CHSE/Pages/statistics.aspx \\
\hline Massachusetts & http://www.mass.gov/eohhs/gov/departments/dph/programs/id/hiv-aids/epi-profile/ \\
\hline Michigan & http://www.michigan.gov/mdhhs/0,5885,7-339-71550_2955_2982_46000_46003---,00.html \\
\hline Minnesota & http://www.health.state.mn.us/divs/idepc/diseases/hiv/stats/index.html \\
\hline Mississippi & http://www.msdh.state.ms.us/msdhsite/index.cfm/14,0,150,134,html \\
\hline Missouri & http://health.mo.gov/data/hivstdaids/data.php \\
\hline Montana & http://dphhs.mt.gov/publichealth/hivstd \\
\hline Nebraska & http://dhhs.ne.gov/publichealth/Pages/dpc_hiv.aspx \\
\hline Nevada & http://dpbh.nv.gov/Programs/HIV-OPHIE/dta/Publications/HIVIAIDS_Surveillance_Program_(HIV-OPHIE)_-_Publications/ \\
\hline New Hampshire & http://www.dhhs.nh.gov/data/index.htm \\
\hline New Jersey & http://www.nj.gov/health/hivstdtb/hiv-aids/statmap.shtml \\
\hline New Mexico & http://nmhealth.org/data/infectious/20/ \\
\hline New York & http://www.health.ny.gov/diseases/aids/general/statistics/index.htm \\
\hline New York City & http://www1.nyc.gov/site/doh/data/data-sets/hiv-aids-surveillance-and-epidemiology-reports.pagel \\
\hline North Carolina & http://epi.publichealth.nc.gov/cd/stds/figures.html \\
\hline North Dakota & http://www.ndhealth.gov/hiv/Data/ \\
\hline Ohio & http://www.odh.ohio.gov/healthstats/disease/hivdata/hivcov.aspx \\
\hline Oklahoma & http://www.ok.gov/health/Disease,_Prevention,_Preparedness/HIV_STD_Service/Fact_Sheets_-_OK_Data/index.html \\
\hline Oregon & http://public.health.oregon.gov/DiseasesConditions/CommunicableDisease/DiseaseSurveillanceData/HIVData/Pages/index.aspx \\
\hline Pennsylvania & http://www.health.pa.gov/My\%20Health/Diseases\%20and\%20Conditions/E-H/HIV\%20And\%20AIDS\%20Epidemiology/Pages/default.aspx\#.Wd5C0miPJPZ \\
\hline Philadelphia & http://www.phila.gov/health/AACO/AACODataResearch.html \\
\hline Puerto Rico & http://www.salud.gov.pr/Estadisticas-Registros-y-Publicaciones/Estadisticas\%20VIH/Forms/Allltems.aspx \\
\hline Republic of Palau & http://www.palauhealth.org/files/MOH\%20Annual\%20Report\%202014.pdf \\
\hline Rhode Island & http://health.ri.gov/publications/bytopic.php?parm=HIV \\
\hline San Francisco & http://www.sfdph.org/dph/files/reports/default.asp \\
\hline South Carolina & http://www.dhec.sc.gov/Health/DiseasesandConditions/InfectiousDiseases/HIVandSTDs/DataandReports/ \\
\hline South Dakota & http://doh.sd.gov/diseases/infectious/HIV-AIDS/Prevention.aspx \\
\hline Tennessee & http://tn.gov/health/topic/STD-surveillance \\
\hline Texas & http://www.dshs.texas.gov/hivstd/reports/default.shtm \\
\hline U.S. Virgin Islands & http://doh.vi.gov/programs/communicable-disease/index.html \\
\hline Utah & http://health.utah.gov/epi/diseases/hivaids/surveillance/index.html \\
\hline Vermont & http://www.healthvermont.gov/immunizations-infectious-disease/hiv/surveillance \\
\hline Virginia & http://www.vdh.virginia.gov/disease-prevention/sexually-transmitted-diseases-std-surveillance-operations-data-administration/hiv-data-statistics/ \\
\hline Washington & http://www.doh.wa.gov/DataandStatisticalReports/DiseasesandChronicConditions/HIVAIDSData.aspx \\
\hline West Virginia & http://www.dhhr.wv.gov/oeps/std-hiv-hep/HIV_AIDS/Pages/HIVSurveillance.aspx \\
\hline Wisconsin & http://www.dhs.wisconsin.gov/aids-hiv/data.htm \\
\hline Wyoming & ttp://health.wyo.gov/publichealth/communicable-disease-unit/hivaids-surveillance-program/ \\
\hline
\end{tabular}

Note. Electronic reports are not available for the following areas: American Samoa and the Northern Mariana Islands. 